\title{
Thioetherification via Photoredox/Nickel Dual Catalysis
}

\author{
Matthieu Jouffroy, Christopher B. Kelly, and Gary A. Molander ${ }^{*}$ \\ Roy and Diana Vagelos Laboratories, Department of Chemistry, University of Pennsylvania, \\ 231 South $34^{\text {th }}$ Street, Philadelphia, Pennsylvania 19104-6323
}

*To whom correspondence should be addressed. E-mail: gmolandr@sas.upenn.edu

\section{Supporting Information}

Key to Abbreviated Terms $\quad$ S2

General Considerations

S2

Comments regarding origins of starting materials, purification of solvents, materials for photoreactors, and spectroscopic techniques.

\section{Photochemical Reactor Design}

Details regarding the design of LED-based photoreactors, instructions for their preparation, and photographs .

Synthesis of Alkylbis(catecholato)silicates

Procedures for the preparation of alkylsilicates used in

thioetherification and spectral characterization information.

Synthesis of $\left[\mathrm{Ru}(\mathrm{bpy})_{3}\right]\left(\mathrm{PF}_{6}\right)_{2}$

S7

Procedure for photocatalyst synthesis.

General Procedure for Thioetherification Using Alkylthiolsilicate 1a

General procedure for synthesis, isolation procedures, and spectral characterization of thioethers.

General Procedure for Thioetherification Using Thiols and Alkylsilicate 1b

General procedure for synthesis, isolation procedures, and spectral characterization of thioethers.

Representative Procedure for Large Scale Thioetherification

General procedure for scale up of the described thioetherifications.

Control and Mechanistic Studies

${ }^{1} \mathrm{H}$ NMR Spectra of Synthesized Compounds

${ }^{13} \mathrm{C}$ NMR Spectra of Synthesized Compounds

${ }^{19}$ F NMR Spectra of Synthesized Compounds 


\section{Key to Abbreviated Terms:}

bpy: 2,2'-bipyridyl

CFL: Compact fluorescent light
LED: Light-emitting diode

dtbbpy: 4,4'-di-tert-butyl-2,2'-dipyridyl

\section{General Considerations:}

\section{General:}

All chemical transformations requiring inert atmospheric conditions or vacuum distillation utilized Schlenk line techniques with a 4- or 5-port dual-bank manifold. Argon or nitrogen was used to provide such an atmosphere. NMR Spectra $\left({ }^{1} \mathrm{H},{ }^{13} \mathrm{C},{ }^{19} \mathrm{~F}\right)$ were performed at $298 \mathrm{~K} .{ }^{1} \mathrm{H}$ NMR spectra were referenced to residual non-deuterated chloroform $(\delta 7.26)$ in $\mathrm{CDCl}_{3}$, residual DMSO- $d_{5}\left(\delta\right.$ 2.50) in DMSO- $d_{6}$, acetone- $d_{5}\left(\delta\right.$ 2.09) in acetone- $d_{6}$, and residual MeCN- $d_{2}(\delta$ $1.94)$ in $\mathrm{MeCN}-d_{3} .{ }^{13} \mathrm{C}$ NMR spectra were referenced to $\mathrm{CDCl}_{3}(\delta 77.30), \mathrm{DMSO}-d_{6}(\delta 39.52)$, the carbonyl carbon of acetone $\left(\delta\right.$ 205.87), or the nitrile carbon of $\mathrm{MeCN}-d_{3}(\delta 118.26)$, respectively. ${ }^{19} \mathrm{~F}$ NMR spectra were referenced to hexafluorobenzene $(\delta-164.9)$ as an internal standard. ${ }^{1}$ Reactions were monitored by HPLC, GC/MS, ${ }^{1} \mathrm{H}$ NMR, and/or by TLC on silica gel plates (60 A porosity, $250 \mu \mathrm{m}$ thickness). TLC analysis was performed using hexanes/EtOAc as the eluant and visualized using permanganate stain, Seebach's stain, ${ }^{2}$ ninhydrin stain, and/or UV light. Silica plugs utilized flash silica gel (60 ^ porosity, 32-63 $\mu \mathrm{m}$ ). Flash chromatography was accomplished using an automated system (visualizing at $254 \mathrm{~nm}$, monitoring at $280 \mathrm{~nm}$ ) with

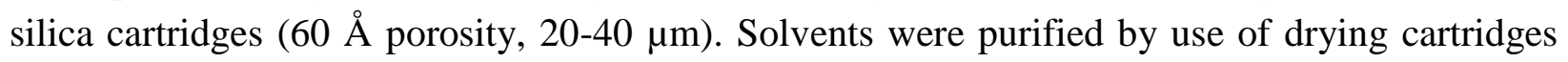
through a solvent delivery system. Melting points $\left({ }^{\circ} \mathrm{C}\right)$ are uncorrected. Optical rotations were recorded using a Jasco P-2000 polarimeter with concentration $(c)$ is in $\mathrm{g} / 100 \mathrm{~mL}$.

\section{Chemicals:}

Deuterated NMR solvents were either used as purchased (DMSO- $d_{6}$ ) or were stored over $4 \AA$ molecular sieves and/or $\mathrm{K}_{2} \mathrm{CO}_{3}\left(\mathrm{CDCl}_{3}\right) . \mathrm{Na}_{2} \mathrm{SO}_{4}, \mathrm{MgSO}_{4}, \mathrm{MeOH}, \mathrm{CH}_{2} \mathrm{Cl}_{2}, \mathrm{CHCl}_{3}, \mathrm{MeCN}$, pentane, $\mathrm{Et}_{2} \mathrm{O}$, and pyridine, were used as purchased. $\mathrm{Et}_{3} \mathrm{~N}$ and $i-\mathrm{Pr}_{2} \mathrm{NH}$, were purchased from commercial suppliers and distilled from $\mathrm{CaH}_{2}$ prior to use. THF was purchased and dried via a solvent delivery system. Catechol was purchased and recrystallized from refluxing hexanes or heptanes. DMF (99.8\%, extra dry) was stored over $4 \AA$ molecular sieves. $\left[\mathrm{NiCl}_{2}(\mathrm{dme})\right]$ (min. $97 \%$ ) and $\mathrm{RuCl}_{3} \cdot 3 \mathrm{H}_{2} \mathrm{O}$ were purchased commercially. Aryl halides were purchased from commercial suppliers and used without further purification. $\left[\mathrm{Ru}(\mathrm{bpy})_{3}\right]\left(\mathrm{PF}_{6}\right)_{2}$ was prepared inhouse by the procedure outlined here. New alkylsilicates were prepared according to the representative procedure outlined here from their corresponding alkyltrimethoxysilanes. Information (preparation protocols, characterization etc.) for silicates $\mathbf{2 d}, \mathbf{2 e}, \mathbf{2 f}, \mathbf{2 g}$, and $\mathbf{2 h}$ can be found in our previous report. ${ }^{3}$

\footnotetext{
${ }^{1}$ Ravikumar, I.; Saha, S.; Ghosh, P. Chem. Commun. 2011, 47, 4721.

${ }^{2}$ Seebach, D.; Imwinkelried, R; Stucky, G. Helv. Chim. Acta 1987, 70, 448.

${ }^{3}$ Jouffroy, M.; Primer, D.; Molander, G. A. J. Am. Chem. Soc., 2015, ASAP DOI: 10.1021/jacs.5b10963
} 


\section{Photochemistry:}

Irradiation of reaction vessels was accomplished either using standard 26 W CFLs or LEDs (blue or white). The choice of light source did not seem to have any effect on reaction success. In most cases either CFL or blue LEDs were employed for irradiation. LEDs were configured as outlined in the Photochemical Reactor Design section. A fan was employed to ensure reactions remained at or near room temperature when using either CFLs or LEDs.

\section{Information for LED-based Photoreactor Components:}

- Blue LEDs: 39.4 inch strips, $470 \mathrm{~nm}$ blue light, $32918 \mathrm{mcd} \mathrm{ft}^{-1}$

- Natural White LEDs: 39.4 inch strips, 380-700 nm, CCT rating: 4000K

- Power Supply: 12V DC CPS series Power Supply - 15 Watt

- Connectors (links power supply to LEDs): LC2 Locking Male Connector CPS Adapter Cable

- Clip Fan: 2-Speed Clip Fan, 6-Inch

- Pyrex crystallizing dishes (125 X $65 \mathrm{~mm})$

- Aluminum foil

- Duct tape 


\section{Photochemical Reactor Design (LEDs only)}

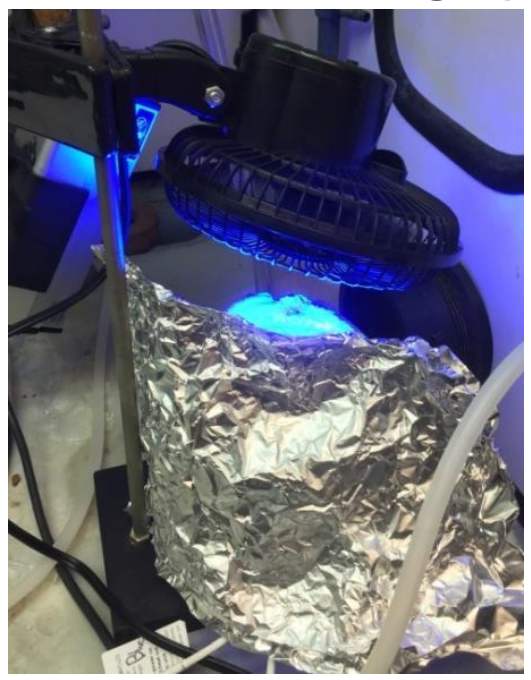

\section{Protocol for reactor setup}

Remove the protective layer on the sticky side of the LED strip and carefully wrap the LED strips on the inside of a clean Pyrex dish. ${ }^{4}$ Four bands of LEDs can fit into a 125 X $65 \mathrm{~mm}$ Pyrex crystallizing dish. ${ }^{5}$ Once the LEDs are securely wrapped, place a layer of aluminium foil around the outside of the dish (including the

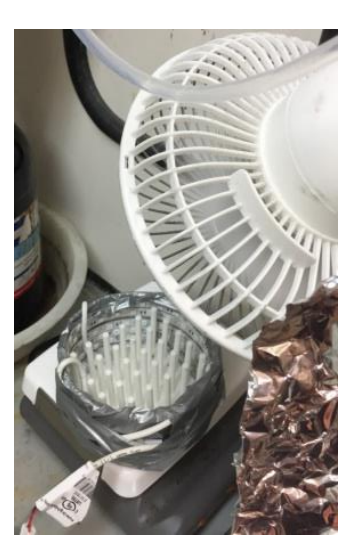
bottom). Tape the connector wires as well as the foil with duct tape to secure both in place. For vial-scale reactions, cut a sample vial rack using a saw and place it inside. For larger vessels (e.g., round bottom flasks), simply lower the flask into the irradiation bay. ${ }^{6}$ Place the reactor on top of a stirring place. Position a fan about 6-12 inches above the reactor for cooling and set it to its maximum setting. Turn on the lights and fan. Allow $15 \mathrm{~min}$ to pass for temperature equilibration. Temperature should be monitored in real time using a temperature probe (or thermometer) to determine the ambient temperature within the reactor. Place a double layer of aluminum foil in front of the reactor to reflect light back into the reactor. CAUTION: Given the brightness of the reactor, it is recommended that impact-resistant sunglasses be used when working with the reactor for eye protection.

\footnotetext{
${ }^{4}$ Starting from the bottom upward affords the easiest approach.

${ }^{5}$ If smaller lengths of LED strips are used, they can be linked together. Most LED strips are able to be cut (at specified locations) and powered by either end. The appropriate connector is required (male or female) for each end. ${ }^{6}$ This design can accommodate up to a $250 \mathrm{~mL}$ round bottom flask. However, if desired, a larger reactor can be assembled by using larger recrystallization dish and additional LEDs.
} 


\section{Synthesis of Alkylbis(catecholato)silicates}

\section{Preparation of Diisopropylammonium Bis(catecholato)(3-mercaptopropyl)silicate (1a)}

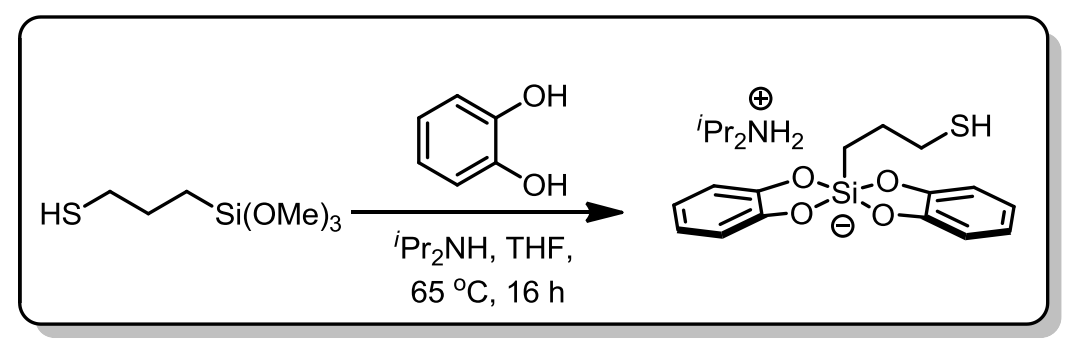

To an oven-dried, $250 \mathrm{~mL}$ round bottom flask equipped with a stir bar, reflux condenser, and gas inlet adapter was added catechol ${ }^{7}(11.74 \mathrm{~g}, 106.6 \mathrm{mmol}, 1.95$ equiv) followed by anhyd THF $(110 \mathrm{~mL})$ and anhyd diisopropylamine ${ }^{8}(6.53 \mathrm{~g}, 9.08 \mathrm{~mL}, 64.6 \mathrm{mmol}, 1.2$ equiv). The mixture was placed under an argon atmosphere and was allowed to stir at $\mathrm{rt}$ for $5 \mathrm{~min}$. The solution became a pale reddish brown. After this time, (3-mercaptopropyl)trimethoxysilane (10.57 g, 53.8 mmol, 1.0 equiv) was added. The solution immediately lightened to a golden yellow. The solution was then heated to reflux in an oil bath and allowed to stir at this temperature overnight. ${ }^{9}$ Once the reaction was judged to be complete by crude ${ }^{1} \mathrm{H}$ NMR analysis, ${ }^{10}$ the solvent was removed in vacuo by rotary evaporation. The resulting powder was collected via filtration through a medium porosity fritted funnel. The powder was washed with $\mathrm{Et}_{2} \mathrm{O}(\sim 100$ $\mathrm{mL}$ ). The solid was collected and dried further in vacuo to give the alkylthiolsilicate 1a containing 0.25 equiv of $\operatorname{THF}^{11}(21.90 \mathrm{~g}, 93 \%)$ as a powdery off-white solid $\left(\mathrm{mp}=135^{\circ} \mathrm{C}\right)$.

${ }^{1} \mathrm{H}$ NMR (MeCN- $\left.d_{3}, 500 \mathrm{MHz}\right) \delta 0.59-0.68(\mathrm{~m}, 2 \mathrm{H}), 1.30(\mathrm{~d}, J=6.4 \mathrm{~Hz}, 12 \mathrm{H}), 1.33$ - 1.41 (br s, $1 \mathrm{H}), 1.51$ (dt, $J=15.4,7.7 \mathrm{~Hz}, 2 \mathrm{H}), 2.35(\mathrm{t}, J=6.6 \mathrm{~Hz}, 2 \mathrm{H}), 3.55$ (spt, $J=6.6 \mathrm{~Hz}, 2 \mathrm{H}), 6.51-6.59$ $(\mathrm{m}, 4 \mathrm{H}), 6.61-6.70(\mathrm{~m}, 4 \mathrm{H}) .{ }^{13} \mathrm{C}$ NMR $\left(\mathrm{MeCN}-d_{3}, 125 \mathrm{MHz}\right) \delta 17.6\left(\mathrm{CH}_{2}\right), 19.3\left(\mathrm{CH}_{3}\right), 28.3$ $\left(\mathrm{CH}_{2}\right), 30.7\left(\mathrm{CH}_{2}\right), 49.3(\mathrm{CH}), 111.1(\mathrm{C}), 118.9(\mathrm{CH}), 151.2(\mathrm{CH})$. FT-IR $\left(\mathrm{cm}^{-1}\right.$, neat, ATR) 3045 (w, br), 2988 (w), 1484 (vs), 1237 (vs), 1013 (w), 811 (vs) 736 (s, br), 517 (w). HRMS (ES-) calcd for $\mathrm{C}_{15} \mathrm{H}_{15} \mathrm{O}_{4} \mathrm{SSi}\left[\mathrm{M}-\mathrm{Et}_{3} \mathrm{NH}\right]^{-}: 319.0460$, found: 319.0467 .

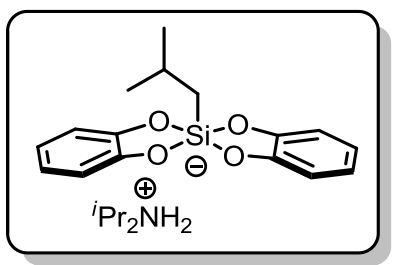

Diisopropylammonium Bis(catecholato)isobutylsilicate, 1 b (4.86 g, $86 \%$ ) was prepared according to the general procedure from isobutyltrimethoxysilane $(2.50 \mathrm{~g}, 0.014 \mathrm{~mol})$ with the following modification: After reaction completion and solvent removal, the crude solid was dissolved in $\mathrm{CH}_{2} \mathrm{Cl}_{2}(\sim 60 \mathrm{~mL})$, and a minimum amount of pentane $(\sim 10 \mathrm{~mL})$ was added as an anti-solvent, resulting in precipitation of a fine white powder. The powder was washed with a

\footnotetext{
${ }^{7}$ Recrystallized from hexane or heptane prior to use.

${ }^{8}$ A similar protocol can be used to prepare the more organic soluble triethylammonium salt. This salt works equally well for photoredox thioetherification.

${ }^{9}$ Depending on the nature of the silicate and its solubility in THF, precipitation of the product would occur.

${ }^{10}$ For DIPA silicates, it is advisable to use acetone- $d_{6}$ or DMSO- $d_{6}$ as the NMR solvent, as these silicates have poor solubility in most other deuterated solvents.

${ }^{11}$ Presence of THF does not impede any photoredox process involving silicates, included thioetherification.
} 
minimal amount of $\mathrm{Et}_{2} \mathrm{O}(\sim 100 \mathrm{~mL})$. followed by a copious amount of pentane $(\sim 125 \mathrm{~mL})$. The desired silicate $\mathbf{1 b}$ was isolated as a powdery white solid $\left(\mathrm{mp}=216{ }^{\circ} \mathrm{C}\right)$.

${ }^{1} \mathrm{H}$ NMR (DMSO- $\left.d_{6}, 500 \mathrm{MHz}\right) \delta 0.50(\mathrm{~d}, J=6.9 \mathrm{~Hz}, 2 \mathrm{H}), 0.75(\mathrm{~d}, J=6.4 \mathrm{~Hz}, 6 \mathrm{H}), 1.20(\mathrm{~d}, J$ $=6.6 \mathrm{~Hz}, 12 \mathrm{H}), 1.68(\mathrm{sept}, J=6.6 \mathrm{~Hz}, 1 \mathrm{H}), 3.35(\mathrm{dt}, J=12.9,6.4 \mathrm{~Hz}, 2 \mathrm{H}), 6.39-6.45(\mathrm{~m}, 4 \mathrm{H})$, 6.47 - 6.55 (m, 4H), 8.02 (br s, $2 \mathrm{H}) .{ }^{13} \mathrm{C}$ NMR (DMSO- $\left.d_{6}, 125 \mathrm{MHz}\right) \delta 18.8\left(\mathrm{CH}_{3}\right), 24.4(\mathrm{CH})$, $26.3\left(\mathrm{CH}_{3}\right), 29.4\left(\mathrm{CH}_{2}\right), 46.4(\mathrm{CH}), 109.4(\mathrm{C}), 117.0(\mathrm{CH}), 150.6(\mathrm{CH})$. FT-IR $\left(\mathrm{cm}^{-1}\right.$, neat, ATR) 3047 (w, br) 2948 (w) 1484 (vs) 1238 (vs) 1014 (w) 812 (w) 734 (vs) 498 (m). HRMS (ES-) calcd for $\mathrm{C}_{16} \mathrm{H}_{17} \mathrm{O}_{4} \mathrm{Si}\left[\mathrm{M}-{ }^{i} \mathrm{Pr}_{2} \mathrm{NH}_{2}\right]^{-}: 301.0896$, found: 301.0899

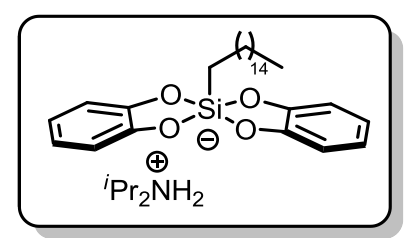

Diisopropylammonium Bis(catecholato)hexadecylsilicate, 1c (5.86 $\mathrm{g}, 71 \%$ ) was prepared according to the general procedure for silicate synthesis from hexadecyltrimethoxysilane $(5.00 \mathrm{~g}, 14.4 \mathrm{mmol})$. The desired silicate 1c was isolated as an off-white powdery solid $(\mathrm{mp}=$ $\left.138^{\circ} \mathrm{C}\right)$.

${ }^{1}$ H NMR (DMSO- $\left.d_{6}, 500 \mathrm{MHz}\right) \delta 0.42-0.53(\mathrm{~m}, 2 \mathrm{H}), 0.85(\mathrm{t}, J=6.9$ $\mathrm{Hz}, 3 \mathrm{H}), 1.05-1.31(\mathrm{~m}, 40 \mathrm{H}), 3.35$ (sept, $J=6.4 \mathrm{~Hz}, 2 \mathrm{H}), 6.39-6.45(\mathrm{~m}, 4 \mathrm{H}), 6.48-6.54(\mathrm{~m}$, 4H), 8.03 (br s, 2H). ${ }^{13} \mathrm{C}$ NMR (DMSO-d, $\left.125 \mathrm{MHz}\right) \delta 13.9\left(\mathrm{CH}_{3}\right), 18.3\left(\mathrm{CH}_{2}\right), 18.8\left(\mathrm{CH}_{3}\right)$, $22.1\left(\mathrm{CH}_{2}\right), 24.2\left(\mathrm{CH}_{2}\right), 28.7\left(\mathrm{CH}_{2}\right), 29.0\left(\mathrm{CH}_{2} \times 9\right), 31.3\left(\mathrm{CH}_{2}\right), 32.8\left(\mathrm{CH}_{2}\right), 46.4(\mathrm{CH}), 109.4$ (C), $116.9(\mathrm{CH}), 150.6(\mathrm{CH})$. FT-IR ( $\mathrm{cm}^{-1}$, neat, ATR) 3048 (w, br), $2988(\mathrm{~m}), 2924(\mathrm{w}), 1486$ (vs), 1239 (vs), 813 (vs), 734 (s), 717 (s, br). HRMS (ES-) calcd for $\mathrm{C}_{28} \mathrm{H}_{41} \mathrm{O}_{4} \mathrm{Si}\left[\mathrm{M}-{ }^{i} \mathrm{Pr}_{2} \mathrm{NH}_{2}\right]^{-}$: 469.2774, found: 469.2770. 


\section{Synthesis of $\left[\mathrm{Ru}(\mathrm{bpy})_{3}\right]\left(\mathrm{PF}_{6}\right)_{2}$ \\ Preparation of the Photocatalyst}

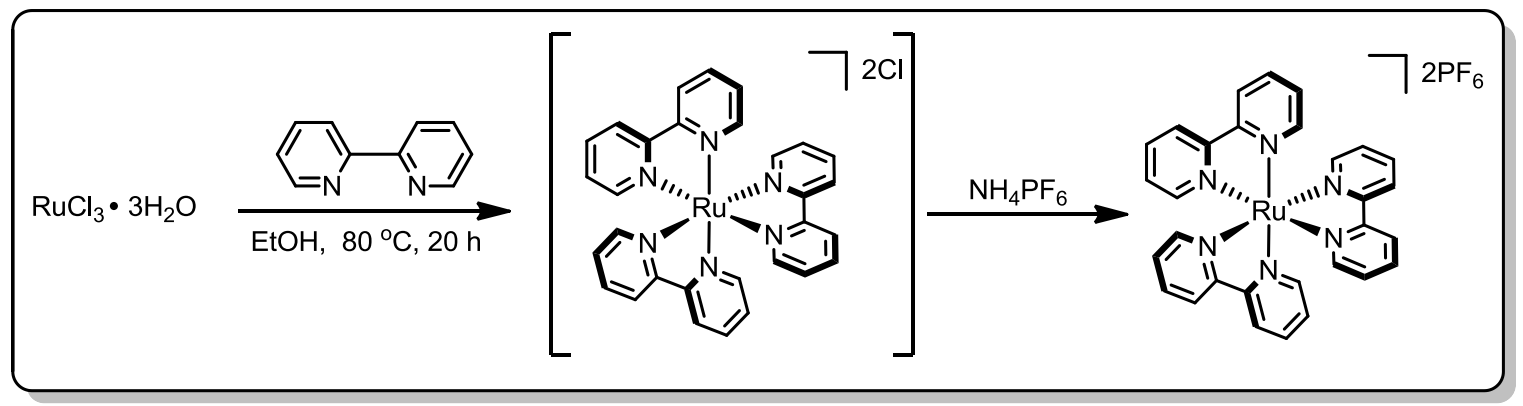

This procedure is a modification of the procedure outlined by Wrighton. ${ }^{12}$ To a $500 \mathrm{~mL}$ round bottom flask equipped with a stir bar and reflux condenser was added 2,2'-bipyridyl (9.68 g, 62.0 mmol, 5.1 equiv) and $\mathrm{RuCl}_{3} \cdot 3 \mathrm{H}_{2} \mathrm{O}(3.16 \mathrm{~g}, 12.1 \mathrm{mmol}, 1.0$ equiv). The system was sealed with a rubber septum and evacuated four times via an inlet needle and purged with $\mathrm{N}_{2}$. Freshly distilled and degassed $\mathrm{EtOH}(300 \mathrm{~mL})$ was then added, and the solution was heated to reflux via an oil bath. The solution was allowed to stir at reflux for $16 \mathrm{~h}$. The flask was then cooled to $\mathrm{rt}$ and $\mathrm{NH}_{4} \mathrm{PF}_{6}$ (16.30 g, $100 \mathrm{mmol}, 8.3$ equiv) was added, resulting in the formation of a voluminous orange precipitate. The reflux condenser was removed, and the solution was heated $15 \mathrm{~min}$ at 40 ${ }^{\circ} \mathrm{C}$. After this time, the solution was cooled to $\mathrm{rt}$ and then chilled in a refrigerator $\left(\approx 5{ }^{\circ} \mathrm{C}\right)$ overnight. The precipitate was collected by vacuum filtration and washed thoroughly with $\mathrm{H}_{2} \mathrm{O}$ $(\sim 1 \mathrm{~L})$, EtOH $(\sim 300 \mathrm{~mL})$, and finally $\mathrm{Et}_{2} \mathrm{O}(\sim 200 \mathrm{~mL})$ to afford a bright red powder. ${ }^{13}{ }^{1} \mathrm{H} \mathrm{NMR}$ analysis of the solid revealed the presence of a small amount of 2,2'-bipyridyl. To purify the photocatalyst further, the red solid was taken up in hot acetone $(400 \mathrm{~mL})$ and filtered through a pad of Celite ${ }^{\circledR}(10 \times 3 \mathrm{~cm})$, eluting with hot acetone $(\sim 300 \mathrm{~mL})$. The resulting pumpkin orange filtrate was concentrated in vacuo by rotary evaporation to $c a .400 \mathrm{~mL}$, then reagent grade $\mathrm{MeOH}(\sim 200 \mathrm{~mL})$ was added. Rapidly, an orange solid formed, and addition of $\mathrm{Et}_{2} \mathrm{O}(\sim 300 \mathrm{~mL})$ further enhances precipitation of the solid. The precipitate was collected by vacuum filtration, and the pumpkin orange cake was washed thoroughly with $\mathrm{EtOH}(\sim 300 \mathrm{~mL})$ and finally $\mathrm{Et}_{2} \mathrm{O}$ $(\sim 200 \mathrm{~mL})$ to afford the title compound as a fluffy powder $(8.07 \mathrm{~g}, 78 \%)$. Characterization data for this compound matched that reported in the literature. ${ }^{25}$

${ }^{1} \mathrm{H}$ NMR (acetone- $\left.d_{6}, 500 \mathrm{MHz}\right) \delta 7.57-7.66(\mathrm{~m}, 6 \mathrm{H}), 8.10(\mathrm{dd}, J=5.6,0.5 \mathrm{~Hz}, 6 \mathrm{H}), 8.25(\mathrm{tt}, J$ $=7.8,1.2 \mathrm{~Hz}, 6 \mathrm{H}), 8.86(\mathrm{dd}, J=8.2,0.6 \mathrm{~Hz}, 6 \mathrm{H}) .{ }^{13} \mathrm{C}$ NMR (acetone- $\left.d_{6}, 125 \mathrm{MHz}\right) \delta 125.0$ $(\mathrm{CH}), 128.5(\mathrm{CH}), 138.6(\mathrm{CH}), 152.4(\mathrm{CH}), 157.8(\mathrm{CH}) .{ }^{19} \mathrm{~F}$ NMR (acetone- $\left.d_{6}, 471 \mathrm{MHz}\right) \delta-$ $73.58(\mathrm{~s}, 6 \mathrm{~F})-72.08(\mathrm{~s}, 6 \mathrm{~F}) .{ }^{31} \mathrm{P}$ NMR (acetone- $\left.d_{6}, 202 \mathrm{MHz}\right) \delta-144.27$ (quin, $J=704.60 \mathrm{~Hz}$ ).

\footnotetext{
${ }^{12}$ Mabrouk, P. A.; Wrighton, M. S. Inorg. Chem. 1986, 25, 526.

${ }^{13}$ In some cases, NMR analysis of the intermediate brick red solid shows the presence of other complexes, namely $\left[\mathrm{Ru}(\mathrm{bpy})_{3}\right] \mathrm{Cl}_{2}$ and $\left[\mathrm{Ru}(\mathrm{bpy})_{3}\right]\left(\mathrm{PF}_{6}\right) \mathrm{Cl}$. In these cases, the solid was retaken up in $\mathrm{H}_{2} \mathrm{O}(\sim 200 \mathrm{~mL})$, and $\mathrm{NH}_{4} \mathrm{PF}_{6}(\sim 2$ equiv) was added. The resulting suspension was sonicated at $\mathrm{rt}$ for $30 \mathrm{~min}$ then filtered, affording a brick red cake that was purified using the above mentioned procedure.
} 


\section{General Procedure for Thioetherification Using Thiolsilicate 1a}

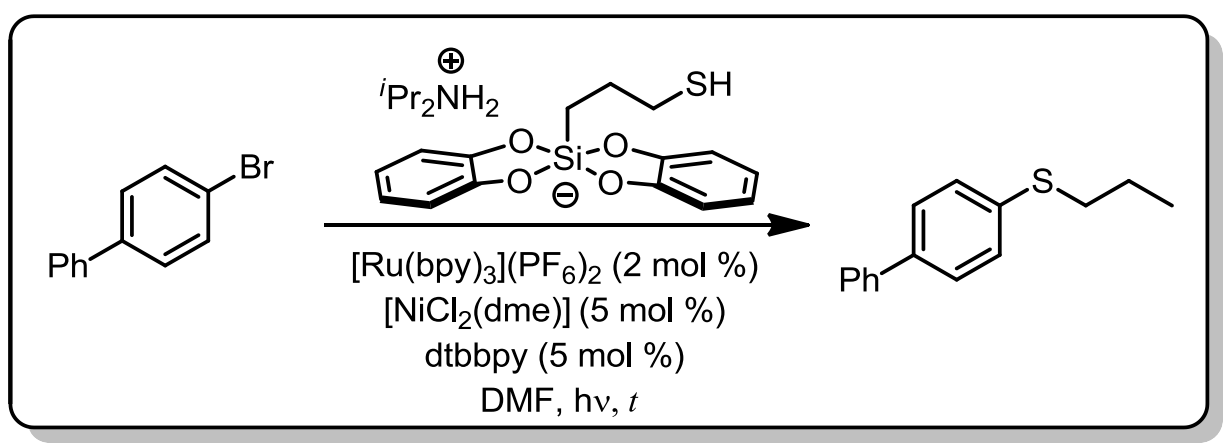

\section{[1,1'-Biphenyl]-4-yl(propyl)sulfane (2a)}

To an $8 \mathrm{~mL}$ reaction vial equipped with an appropriately sized stir bar were added dtbbpy (6.7 $\mathrm{mg}, 0.025,0.05$ equiv) and $\left[\mathrm{NiCl}_{2}(\mathrm{dme})\right](5.5 \mathrm{mg}, 0.025 \mathrm{mmol}, 0.05$ equiv). The vial was sealed with a cap containing a TFE lined silicone septa and placed under a $\mathrm{N}_{2}$ atmosphere via an inlet needle. The vial was charged with $\sim 1.5 \mathrm{~mL}$ of anhyd THF and the resulting suspension was heated briefly with a heat gun until a homogenous pale green solution was observed. The solution was cooled in an ice water bath, resulting in the immediate precipitation of an evergreen solid. The solution was then evaporated in vacuo to give the ligated nickel complex. ${ }^{14}$ The cap was removed from the vial and alkylthiolsilicate $1 \mathrm{a}(253 \mathrm{mg}, 0.60 \mathrm{mmol}, 1.2$ equiv), 4-bromo1,1'-biphenyl (117 mg, $0.50 \mathrm{mmol}, 1$ equiv), ${ }^{15}$ and $\left[\mathrm{Ru}(\mathrm{bpy})_{3}\right]\left(\mathrm{PF}_{6}\right)(8.6 \mathrm{mg}, 0.01 \mathrm{mmol}, 0.02$ equiv) were added. The vial was resealed and was evacuated three times via an inlet needle then purged with inert gas. The vial was then charged with dissolved in anhyd, degassed DMF (5 mL) via a syringe. The cap was sealed with Parafilm ${ }^{\circledR}$, and the now bright red solution was irradiated in the aforementioned LED reactor. The temperature of the reaction was maintained at approximately $27{ }^{\circ} \mathrm{C}$ via a fan. The solution was stirred vigorously while being irradiated. Reaction progress was monitored by HPLC and/or GC/MS. The now opaque, milky-brown solution was poured in a separatory funnel and diluted with saturated aqueous $\mathrm{Na}_{2} \mathrm{CO}_{3}(20 \mathrm{~mL})^{16}$ and $\mathrm{Et}_{2} \mathrm{O}^{17}(\sim 20 \mathrm{~mL})$. The layers were separated, ${ }^{18}$ and the aqueous layer was extracted with $\mathrm{Et}_{2} \mathrm{O}(3 \times \sim 20 \mathrm{~mL})$. The combined organic layers were washed with saturated aqueous $\mathrm{Na}_{2} \mathrm{CO}_{3}$ $(2 \times \sim 30 \mathrm{~mL})$, deionized $\mathrm{H}_{2} \mathrm{O}(\sim 30 \mathrm{~mL})$, and brine $(\sim 50 \mathrm{~mL}) .{ }^{19}$ The organic layer was dried $\left(\mathrm{MgSO}_{4}\right)$, and the solvent was removed in vacuo by rotary evaporation. Further purification was accomplished by $\mathrm{SiO}_{2}$ column chromatography (gradient hexane/EtOAc) to give the desired thioether $2 \mathbf{a}$ as a white powder $(83 \mathrm{mg}, 73 \%)\left(\mathrm{mp}=69^{\circ} \mathrm{C}\right)$.

\footnotetext{
${ }^{14}$ We have performed the reaction with and without pre-ligation of the ligand. We found that there is no effect on the yield of cross couplings performed here. However, it was necessary for certain systems in our previous report, see Ref 3.

${ }^{15}$ Liquid bromides were added later as a solution in DMF

${ }^{16}$ Alternatively $2 \mathrm{M} \mathrm{NaOH}$ may be used

${ }^{17}$, EtOAc was used in place of $\mathrm{Et}_{2} \mathrm{O}$ for the extractions when isolating products containing polar functional groups.

${ }^{18}$ Note that a precipitate will often form and rest at the interface between the organic and aqueous layers. It can be discarded during the washes without compromising yield.

${ }^{19}$ In certain cases, specifically when working with systems that did not contain basic moieties, an optionally four wash can be added. Following the saturated aqueous $\mathrm{Na}_{2} \mathrm{CO}_{3}$ (or $2 \mathrm{M} \mathrm{NaOH}$ ) wash, a $2 \mathrm{M} \mathrm{HCl}(\sim 30 \mathrm{~mL}) \mathrm{may} \mathrm{be}$ used. This will remove any residual dtbbpy.
} 
${ }^{1} \mathrm{H}$ NMR $\left(\mathrm{CDCl}_{3}, 500 \mathrm{MHz}\right) \delta 1.08(\mathrm{t}, J=7.3 \mathrm{~Hz}, 3 \mathrm{H}), 1.70-1.78(\mathrm{~m}, 2 \mathrm{H}), 2.97(\mathrm{t}, J=7.3 \mathrm{~Hz}$, 2H), $7.36(\mathrm{tt}, J=7.3,1.7 \mathrm{~Hz}, 1 \mathrm{H}), 7.40-7.48(\mathrm{~m}, 4 \mathrm{H}), 7.54(\mathrm{~d}, J=8.6 \mathrm{~Hz}, 2 \mathrm{H}), 7.58-7.62(\mathrm{~m}$, 2H). ${ }^{13} \mathrm{C} \mathrm{NMR}\left(\mathrm{CDCl}_{3}, 125 \mathrm{MHz}\right) \delta 13.7\left(\mathrm{CH}_{3}\right), 22.8\left(\mathrm{CH}_{2}\right), 35.9\left(\mathrm{CH}_{2}\right), 127.1(\mathrm{CH}), 127.5$ $(\mathrm{CH}), 127.7(\mathrm{CH}), 129.1(\mathrm{CH}), 129.5(\mathrm{CH}), 136.4(\mathrm{C}), 138.9(\mathrm{C}), 140.8(\mathrm{C})$. GC-MS (EI) 228 $\left([\mathrm{M}]^{+}, 100 \%\right), 199(11 \%), 185(62 \%), 166(34 \%), 152(30 \%), 141(6 \%), 115(8 \%) 102(2 \%), 77$ (3\%), 63 (2\%). FT-IR ( $\mathrm{cm}^{-1}$, neat, ATR) 3055 (vw), 3032 (vw), $2963(\mathrm{w}), 2932(\mathrm{w}), 2872(\mathrm{vw})$, 1593 (w), 1477 (m), 1098 (m), 823 (m), 752 (vs), 685 (s). HRMS (CI+) calcd for $\mathrm{C}_{15} \mathrm{H}_{16} \mathrm{~S}[\mathrm{M}]^{+}$: 228.0973, found: 228.0971.

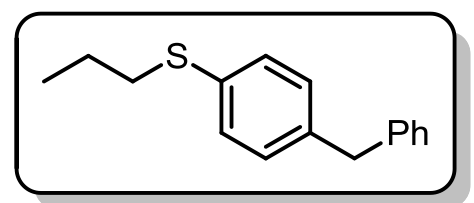

(4-Benzylphenyl)(propyl)sulfane, 2b (118 $\mathrm{mg}, \quad 97 \%)$ was prepared according to the general procedure for thioetherification from 1-benzyl-4-bromobenzene $(123 \mathrm{mg}, 0.5 \mathrm{mmol})$. The desired thioether was obtained as a clear, colorless oil.

${ }^{1} \mathrm{H} \mathrm{NMR}\left(\mathrm{CDCl}_{3}, 500 \mathrm{MHz}\right) \delta 1.06(\mathrm{t}, J=7.3 \mathrm{~Hz}, 3 \mathrm{H}), 1.66-1.74$ $(\mathrm{m}, 2 \mathrm{H}), 2.91(\mathrm{t}, J=7.3 \mathrm{~Hz}, 2 \mathrm{H}), 3.99(\mathrm{~s}, 2 \mathrm{H}), 7.15(\mathrm{~d}, J=8.1 \mathrm{~Hz}, 2 \mathrm{H}), 7.20-7.28(\mathrm{~m}, 3 \mathrm{H})$, 7.29 - $7.36(\mathrm{~m}, 4 \mathrm{H}) .{ }^{13} \mathrm{C}$ NMR $\left(\mathrm{CDCl}_{3}, 125 \mathrm{MHz}\right) \delta 13.7\left(\mathrm{CH}_{3}\right), 22.8\left(\mathrm{CH}_{2}\right), 36.3\left(\mathrm{CH}_{2}\right), 41.7$ $\left(\mathrm{CH}_{2}\right), 126.4(\mathrm{CH}), 128.7(\mathrm{CH}), 129.2(\mathrm{CH}), 129.7(\mathrm{CH}), 129.8(\mathrm{CH}), 134.5(\mathrm{C}), 139.2(\mathrm{C})$, 141.2 (C). GC-MS (EI) 242 ([M] $\left.{ }^{+}, 100 \%\right), 213$ (16\%), 200 (17\%), 179 (8\%), 167 (76\%), 152 (16\%), 91 (48\%). FT-IR (cm-1, neat, ATR) 3061 (vw), 3032 (vw), $2961(\mathrm{w}), 2929$ (w), 1492 (m), 1453(w), 1092, 788 (m) 745 (s), 696 (vs). HRMS (CI+) calcd for $\mathrm{C}_{16} \mathrm{H}_{18} \mathrm{~S}[\mathrm{M}]^{+}$: 242.1129, found: 242.1128 .

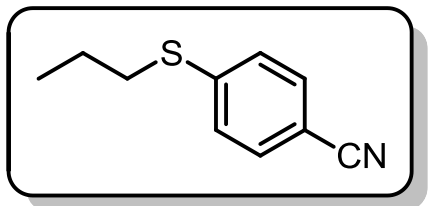

4-(Propylthio)benzonitrile, ${ }^{20}$ 2c $(68 \mathrm{mg}, 77 \%)$ was prepared according to the general procedure for thioetherification from 4bromobenzonitrile (91 $\mathrm{mg}, 0.5 \mathrm{mmol})$. The desired thioether was obtained as a clear, pale yellow oil.

${ }^{1} \mathrm{H}$ NMR $\left(\mathrm{CDCl}_{3}, 500 \mathrm{MHz}\right) \delta 1.06(\mathrm{t}, J=7.3 \mathrm{~Hz}, 3 \mathrm{H}), 1.69-1.77$ $(\mathrm{m}, 2 \mathrm{H}), 2.96(\mathrm{t}, J=7.3 \mathrm{~Hz}, 2 \mathrm{H}), 7.29(\mathrm{~d}, J=8.2 \mathrm{~Hz}, 2 \mathrm{H}), 7.52(\mathrm{~d}, J=8.5 \mathrm{~Hz}, 2 \mathrm{H}) .{ }^{13} \mathrm{C}$ NMR $\left(\mathrm{CDCl}_{3}, 125 \mathrm{MHz}\right) \delta 13.7\left(\mathrm{CH}_{3}\right), 22.3\left(\mathrm{CH}_{2}\right), 34.1\left(\mathrm{CH}_{2}\right), 108.1(\mathrm{C}), 119.2(\mathrm{C}), 127.0(\mathrm{CH})$, $132.4(\mathrm{CH}), 145.5(\mathrm{C})$. GC-MS (EI) $179\left([\mathrm{M}]^{+}, 79 \%\right), 148$ (43\%), 135 (100\%), 108 (6\%), 104 (7\%), 91 (7\%), 76 (4\%), 63 (5\%). FT-IR ( $\mathrm{cm}^{-1}$, neat, ATR) $2964(\mathrm{w}), 2225(\mathrm{~m}), 1593(\mathrm{~s}), 1485$ (m), 1088 (vs), 818 (vs), 542 (vs). HRMS (ES+) calcd for $\mathrm{C}_{10} \mathrm{H}_{12} \mathrm{NS}[\mathrm{M}+\mathrm{H}]^{+}:$178.0690, found: 178.0692 .

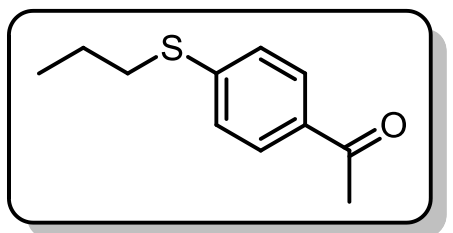

1-(4-(Propylthio)phenyl)ethanone, ${ }^{21}$ 2d $\quad(74 \quad \mathrm{mg}, \quad 76 \%)$ was prepared according to the general procedure for thioetherification from 4-bromoacetophenone ( $95 \mathrm{mg}, 0.5 \mathrm{mmol}$ ). The desired thioether was obtained as a clear, pale yellow oil which solidified upon standing to a white, waxy solid $\left(\mathrm{mp}=36^{\circ} \mathrm{C}\right)$.

${ }^{1} \mathrm{H}$ NMR $\left(\mathrm{CDCl}_{3}, 500 \mathrm{MHz}\right) \delta 1.06(\mathrm{t}, J=7.3 \mathrm{~Hz}, 3 \mathrm{H}), 1.69-1.77$ $(\mathrm{m}, 2 \mathrm{H}), 2.55-2.56(\mathrm{~m}, 3 \mathrm{H}), 2.97(\mathrm{t}, J=7.3 \mathrm{~Hz}, 2 \mathrm{H}), 7.30(\mathrm{~d}, J=8.2 \mathrm{~Hz}, 2 \mathrm{H}), 7.84(\mathrm{~d}, J=8.2$ $\mathrm{Hz}, 2 \mathrm{H}) .{ }^{13} \mathrm{C} \mathrm{NMR}\left(\mathrm{CDCl}_{3}, 125 \mathrm{MHz}\right) \delta 13.8\left(\mathrm{CH}_{3}\right), 22.5\left(\mathrm{CH}_{2}\right), 26.7\left(\mathrm{CH}_{3}\right), 34.3\left(\mathrm{CH}_{2}\right), 126.6$ (CH), $129.0(\mathrm{CH}), 134.1$ (C), 145.2 (C), $197.4(\mathrm{C})$. GC-MS (EI) 194 ([M]+, 88\%), 179 (100\%),

\footnotetext{
${ }^{20}$ Blackaby, W. P. et al. WO 2006134341, 2006.

${ }^{21}$ Cutler, R. A.; Stenger, R. J.; Suter, C. M. J. Am. Chem. Soc., 1952, 74, 5475.
} 
137 (71\%), 123 (13\%), 109 (20\%), 69 (6\%). FT-IR (cm ${ }^{-1}$, neat, ATR) 2967 (w), 1673 (vs), 1587 (s) 1356 (s), 1264 (s), 1180 (s), 1096 (s), 956 (s), 813 (vs), 745 (m), 586 (s). HRMS (CI+) calcd for $\mathrm{C}_{11} \mathrm{H}_{14} \mathrm{OS}[\mathrm{M}]^{+}:$194.0765, found: 194.0762.

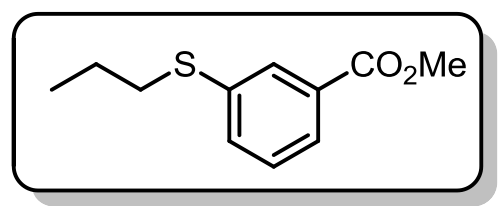

Methyl 3-(Propylthio)benzoate, 2 f (89 mg, 85\%) was prepared according to the general procedure for thioetherification from methyl 3-bromobenzoate (108 $\mathrm{mg}, 0.5 \mathrm{mmol})$. The desired thioether was obtained as a light yellow oil.

${ }^{1} \mathrm{H}$ NMR $\left(\mathrm{CDCl}_{3}, 500 \mathrm{MHz}\right) \delta 1.04(\mathrm{t}, J=7.3 \mathrm{~Hz}, 3 \mathrm{H}), 1.64-$ $1.73(\mathrm{~m}, 2 \mathrm{H}), 2.94(\mathrm{t}, J=7.6 \mathrm{~Hz}, 2 \mathrm{H}), 3.92(\mathrm{~s}, 3 \mathrm{H}), 7.34(\mathrm{t}, J=7.6 \mathrm{~Hz}, 1 \mathrm{H}), 7.49$ (ddd, $J=7.9$, 2.0, $1.1 \mathrm{~Hz}, 1 \mathrm{H}), 7.82(\mathrm{dt}, J=7.8,1.2 \mathrm{~Hz}, 1 \mathrm{H}), 7.98(\mathrm{t}, J=1.7 \mathrm{~Hz}, 1 \mathrm{H}) .{ }^{13} \mathrm{C} \mathrm{NMR}\left(\mathrm{CDCl}_{3}, 125\right.$ $\mathrm{MHz}) \delta 13.7\left(\mathrm{CH}_{3}\right), 22.7\left(\mathrm{CH}_{2}\right), 35.7\left(\mathrm{CH}_{2}\right), 52.5\left(\mathrm{CH}_{3}\right), 127.0(\mathrm{CH}), 129.0(\mathrm{CH}), 129.7(\mathrm{CH})$, $131.1(\mathrm{C}), 133.3(\mathrm{CH}), 138.2$ (C), 167.0 (C). GC-MS (EI) $210\left([\mathrm{M}]^{+}, 100 \%\right), 181(31 \%), 168$ (70\%), 151 (10\%), 149 (11\%), 137 (54\%), 121 (9\%), 109 (21\%), 91 (9\%), 69 (7\%), 59 (8\%). FT-IR (cm ${ }^{-1}$, neat, ATR) 2961 (w), 2930 (w), 1722 (vs), 1436 (m), 1281 (s), 1260 (vs), 1124 (m), 745 (s). HRMS (CI+) calcd for $\mathrm{C}_{11} \mathrm{H}_{14} \mathrm{O}_{2} \mathrm{~S}[\mathrm{M}]^{+}: 210.0715$, found: 210.0719 .

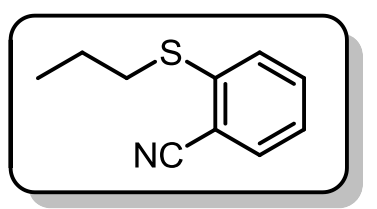

2-(Propylthio)benzonitrile, ${ }^{22} \mathbf{2 g}$ (74 $\left.\mathrm{mg}, 84 \%\right)$ was prepared according to the general procedure for thioetherification from 2-bromobenzonitrile (91 mg, $0.5 \mathrm{mmol}$ ). The desired thioether was obtained as a clear, yellow oil.

${ }^{1} \mathrm{H}$ NMR $\left(\mathrm{CDCl}_{3}, 500 \mathrm{MHz}\right) \delta 1.05(\mathrm{t}, J=7.3 \mathrm{~Hz}, 3 \mathrm{H}), 1.67-1.75(\mathrm{~m}$, 2H), 2.99 (t, $J=7.2 \mathrm{~Hz}, 2 \mathrm{H}), 7.24(\mathrm{t}, J=7.5 \mathrm{~Hz}, 1 \mathrm{H}), 7.41(\mathrm{~d}, J=7.9 \mathrm{~Hz}, 1 \mathrm{H}), 7.49(\mathrm{t}, J=7.3$ $\mathrm{Hz}, 1 \mathrm{H}), 7.60(\mathrm{~d}, J=7.6 \mathrm{~Hz}, 1 \mathrm{H}) .{ }^{13} \mathrm{C} \mathbf{N M R}\left(\mathrm{CDCl}_{3}, 125 \mathrm{MHz}\right) \delta 13.6\left(\mathrm{CH}_{3}\right), 22.5\left(\mathrm{CH}_{2}\right), 35.8$ $\left(\mathrm{CH}_{2}\right), 113.8(\mathrm{C}), 117.5(\mathrm{C}), 126.0(\mathrm{CH}), 129.1(\mathrm{CH}), 133.0(\mathrm{CH}), 133.9(\mathrm{CH}), 142.4(\mathrm{C}) . \mathrm{GC}-$ MS (EI) 177 ([M] $\left.]^{+}, 37 \%\right), 148(13 \%), 135(100 \%), 108(8 \%), 91(6 \%), 76(3 \%) 69(3 \%)$. FT-IR $\left(\mathrm{cm}^{-1}\right.$, neat, ATR) 2964 (w), 2222 (w), $1585(\mathrm{w}), 1463(\mathrm{~m}), 1433$ (m), 753 (vs). HRMS (ES+) calcd for $\mathrm{C}_{10} \mathrm{H}_{12} \mathrm{NS}[\mathrm{M}+\mathrm{H}]^{+}:$178.0690, found: 178.0695 .

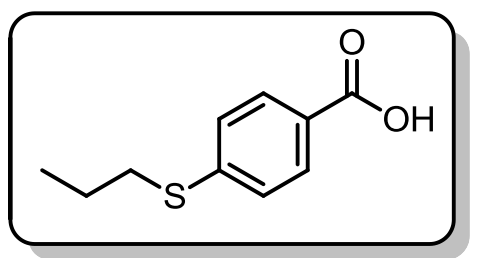

4-(Propylthio)benzoic acid, 2h (75 $\mathrm{mg}, 87 \%)$ was prepared according to the general procedure for thioetherification from 4bromobenzoic acid (101 $\mathrm{mg}, 0.5 \mathrm{mmol}$ ) with the following modified workup: The reaction mixture was quenched with saturated aqueous $\mathrm{NH}_{4} \mathrm{Cl}$. The quenched reaction mixture was extracted with EtOAc $(3 \times 20 \mathrm{~mL})$. The solvent was removed in vacuo by rotary evaporation, giving a brown oil. The brown oil was taken up in $\sim 40 \mathrm{~mL}^{\circ} \mathrm{Et}_{2} \mathrm{O}$ and extracted with saturated aqueous $\mathrm{NaHCO}_{3}(3 \times 20 \mathrm{~mL})$. The combined aqueous layers were mixed with $\sim 40 \mathrm{~mL}$ of EtOAc and acidified by dropwise addition of concentrated $\mathrm{HCl}$ to $\mathrm{pH} 1$ $(\sim 10 \mathrm{~mL})$. The layers were separated, and the aqueous layer was extracted with EtOAc $(3 \times 20$ $\mathrm{mL})$. The combined organic layers were washed with deionized $\mathrm{H}_{2} \mathrm{O}(\sim 60 \mathrm{~mL})$ and dried $\left(\mathrm{MgSO}_{4}\right)$. The solvent was removed in vacuo by rotary evaporation. The desired thioether was obtained as a fluffy off-white powder $\left(\mathrm{mp}=119^{\circ} \mathrm{C}\right)$.

\footnotetext{
${ }^{22}$ Murata, T. et al. EP 2842946, 2015.
} 
${ }^{1} \mathrm{H} \mathrm{NMR}\left(\mathrm{CDCl}_{3}, 500 \mathrm{MHz}\right) \delta 1.07(\mathrm{t}, J=7.3 \mathrm{~Hz}, 3 \mathrm{H}), 1.68-1.81(\mathrm{~m}, 2 \mathrm{H}), 2.98(\mathrm{t}, J=7.2 \mathrm{~Hz}$,

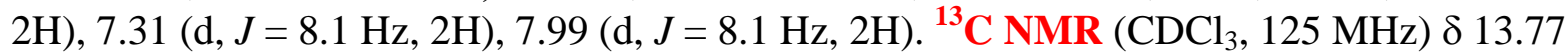

$\left(\mathrm{CH}_{3}\right), 22.43\left(\mathrm{CH}_{2}\right), 34.18\left(\mathrm{CH}_{2}\right), 125.82(\mathrm{C}), 126.45(\mathrm{CH}), 130.77\left(\mathrm{CH}_{2}\right), 146.17(\mathrm{C}), 172.29$

(C). FT-IR (cm ${ }^{-1}$, neat, ATR) 3132 - 2745 (m, very br), 2654 (w), 2528 (m), 1667 (vs), 1591 (s), 1418 (s), 1181 (m), 1091 (s), 954 (s), 847 (m), 761 (s), 549 (m). HRMS (ES-) calcd for $\mathrm{C}_{10} \mathrm{H}_{11} \mathrm{O}_{2} \mathrm{~S}[\mathrm{M}-\mathrm{H}]^{-}: 195.0480$, found: 195.0486 .

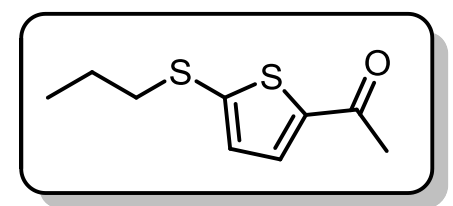

1-(5-(Propylthio)thiophen-2-yl)ethanone, 2i (62 $\mathrm{mg}, 62 \%)$ was prepared according to the general procedure for thioetherification from 2-acetyl-5-bromothiophene (102.5 mg, $0.5 \mathrm{mmol})$. The desired thioether was obtained as a clear, pale yellow oil.

${ }^{1} \mathrm{H}$ NMR $\left(\mathrm{CDCl}_{3}, 500 \mathrm{MHz}\right) \delta 1.03(\mathrm{t}, J=7.3 \mathrm{~Hz}, 3 \mathrm{H}), 1.68-1.76$ $(\mathrm{m}, 2 \mathrm{H}), 2.50(\mathrm{~s}, 3 \mathrm{H}), 2.94(\mathrm{t}, J=7.3 \mathrm{~Hz}, 2 \mathrm{H}), 6.97(\mathrm{~d}, J=4.0 \mathrm{~Hz}, 1 \mathrm{H}), 7.52(\mathrm{~d}, J=3.7 \mathrm{~Hz}, 1 \mathrm{H})$. ${ }^{13} \mathrm{C} \mathrm{NMR}\left(\mathrm{CDCl}_{3}, 125 \mathrm{MHz}\right) \delta 13.3\left(\mathrm{CH}_{3}\right), 22.8\left(\mathrm{CH}_{2}\right), 26.5\left(\mathrm{CH}_{3}\right), 39.5\left(\mathrm{CH}_{2}\right), 129.6(\mathrm{CH})$, $133.0(\mathrm{CH}), 144.5$ (C), 148.2 (C), 189.7 (C). GC-MS (EI) 200 ([M] $\left.{ }^{+}, 100 \%\right), 185$ (22\%), 158 (49\%), 143 (96\%), $114(11 \%), 71(11 \%)$. FT-IR $\left(\mathrm{cm}^{-1}\right.$, neat, ATR) $3078(\mathrm{vw}), 2963(\mathrm{w}), 1652$ (vs), 1407 (vs), 1313 (s), 1269 (vs), 996 (m), 799 (m), 603 (s). HRMS (ES+) calcd for $\mathrm{C}_{9} \mathrm{H}_{13} \mathrm{OS}_{2}$ $[\mathrm{M}+\mathrm{H}]^{+}:$201.0408, found: 201.0412 .

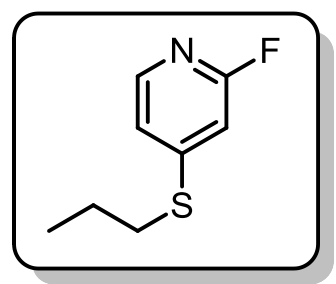

2-Fluoro-4-(propylthio)pyridine, $2 \mathbf{j}$ (62 mg, 72\%) was prepared according to the general procedure for thioetherification from 4-bromo-2fluoropyridine ( $88 \mathrm{mg}, 0.5 \mathrm{mmol})$. The desired thioether was obtained as a clear, colorless oil.

${ }^{1} \mathrm{H} \mathrm{NMR}\left(\mathrm{CDCl}_{3}, 500 \mathrm{MHz}\right) \delta 1.08(\mathrm{t}, J=7.3 \mathrm{~Hz}, 3 \mathrm{H}), 1.72-1.80(\mathrm{~m}$, 2H), $2.96(\mathrm{t}, J=7.3 \mathrm{~Hz}, 2 \mathrm{H}), 6.70(\mathrm{t}, J=1.2 \mathrm{~Hz}, 1 \mathrm{H}), 6.97(\mathrm{dt}, J=5.5$, $1.7 \mathrm{~Hz}, 1 \mathrm{H}), 7.99(\mathrm{~d}, J=5.5 \mathrm{~Hz}, 1 \mathrm{H}) .{ }^{13} \mathrm{C} \mathrm{NMR}\left(\mathrm{CDCl}_{3}, 125 \mathrm{MHz}\right)$ $\delta 13.7\left(\mathrm{CH}_{3}\right), 22.1\left(\mathrm{CH}_{2}\right), 33.1\left(\mathrm{CH}_{2}\right), 105.5\left(\mathrm{~d}, J_{\mathrm{C}-\mathrm{C}-\mathrm{F}}=40.9 \mathrm{~Hz}, \mathrm{CH}\right), 118.8\left(\mathrm{~d}, J_{\mathrm{C}-\mathrm{C}-\mathrm{C}-\mathrm{C}-\mathrm{F}}=3.6\right.$ $\mathrm{Hz}, \mathrm{CH}), 147.1\left(\mathrm{~d}, J_{\mathrm{C}-\mathrm{N}-\mathrm{C}-\mathrm{F}}=16.3 \mathrm{~Hz}, \mathrm{CH}\right), 155.5\left(\mathrm{~d}, J_{\mathrm{C}-\mathrm{C}-\mathrm{C}-\mathrm{F}}=9.1 \mathrm{~Hz}, \mathrm{C}\right), 164.3\left(\mathrm{~d}, J_{\mathrm{C}-\mathrm{F}}=238.9\right.$ $\mathrm{Hz}, \mathrm{CF}) .{ }^{19} \mathrm{~F}$ NMR $\left(\mathrm{CDCl}_{3}, 282 \mathrm{MHz}\right) \delta-71.08$ (s, 1F). GC-MS (EI) $171\left([\mathrm{M}]^{+}, 90 \%\right), 142$ (44\%), 129 (100\%), $122(8 \%), 101(7 \%), 85(10 \%), 69(22 \%), 57(8 \%)$. FT-IR $\left(\mathrm{cm}^{-1}\right.$, neat, ATR) 2966 (w), 1588 (vs), 1540 (s), 1396 (vs), 1088 (w), 900 (vs), 819 (s), 703 (w). HRMS (CI+) calcd for $\mathrm{C}_{8} \mathrm{H}_{11} \mathrm{FNS}[\mathrm{M}+\mathrm{H}]^{+}:$172.0591, found: 172.0593 .

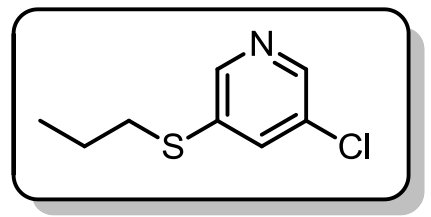

3-Chloro-5-(propylthio)pyridine, ${ }^{23} \mathbf{2 k}(68 \mathrm{mg}, 72 \%)$ was prepared according to the general procedure for thioetherification from 3bromo-5-chloropyridine $(96 \mathrm{mg}, 0.5 \mathrm{mmol}$ ). The desired thioether was obtained as a clear, colorless oil.

${ }^{1} \mathrm{H} \mathrm{NMR}\left(\mathrm{CDCl}_{3}, 500 \mathrm{MHz}\right) \delta 1.05(\mathrm{t}, J=7.3 \mathrm{~Hz}, 3 \mathrm{H}), 1.66-1.74$ $(\mathrm{m}, 2 \mathrm{H}), 2.93(\mathrm{t}, J=7.2 \mathrm{~Hz}, 2 \mathrm{H}), 7.59(\mathrm{t}, J=2.0 \mathrm{~Hz}, 1 \mathrm{H}), 8.35(\mathrm{~d}, J=1.8 \mathrm{~Hz}, 1 \mathrm{H}), 8.41(\mathrm{~d}, J=$ $1.5 \mathrm{~Hz}, 1 \mathrm{H}) .{ }^{13} \mathrm{C}$ NMR $\left(\mathrm{CDCl}_{3}, 125 \mathrm{MHz}\right) \delta 13.6\left(\mathrm{CH}_{3}\right), 22.6\left(\mathrm{CH}_{2}\right), 35.5\left(\mathrm{CH}_{2}\right), 132.2(\mathrm{C})$, $135.5(\mathrm{CH}), 136.0(\mathrm{C}), 145.7(\mathrm{CH}), 147.4(\mathrm{CH})$. GC-MS (EI) $189\left([\mathrm{M}]^{+},{ }^{37} \mathrm{Cl}, 38 \%\right), 187\left([\mathrm{M}]^{+}\right.$, $\left.{ }^{37} \mathrm{Cl}, 100 \%\right), 160\left({ }^{37} \mathrm{Cl} \mathrm{13 \%}\right), 158\left({ }^{35} \mathrm{Cl} \mathrm{36 \%}\right), 147\left({ }^{37} \mathrm{Cl} 34 \%\right), 145\left({ }^{35} \mathrm{Cl}, 96 \%\right), 118(12 \%), 114$ (8\%), 112 (9\%), 101 (12\%), 82 (13\%), 76(8\%), 73 (16\%). FT-IR ( $\mathrm{cm}^{-1}$, neat, ATR) 3040 (vw),

${ }^{23}$ Josey, A. D. et al. US 4683091, 1987. 
2963 (w), 1554 (m), 1404 (m) 1105 (s), 809 (vs), 693 (vs). HRMS (CI+) calcd for $\mathrm{C}_{8} \mathrm{H}_{10} \mathrm{ClNS}$ $[\mathrm{M}]^{+}:$187.0222, found: 187.0222 .

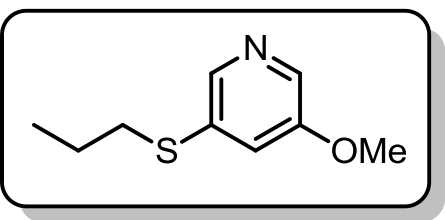

3-Methoxy-5-(propylthio)pyridine, $2 \mathrm{l}$ (67 mg, 73\%) was prepared according to the general procedure for thioetherification from 3bromo-5-methoxypyridine (94 mg, $0.5 \mathrm{mmol}$ ). The desired thioether was obtained as a clear, yellow oil.

${ }^{1} \mathrm{H} \mathrm{NMR}\left(\mathrm{CDCl}_{3}, 500 \mathrm{MHz}\right) \delta 1.02(\mathrm{t}, J=7.3 \mathrm{~Hz}, 3 \mathrm{H}), 1.63-1.71$ (m, 2H), $2.90(\mathrm{t}, J=7.2 \mathrm{~Hz}, 2 \mathrm{H}), 3.83(\mathrm{~s}, 3 \mathrm{H}), 7.13(\mathrm{t}, J=2.2 \mathrm{~Hz}, 1 \mathrm{H}), 8.09(\mathrm{~d}, J=2.7 \mathrm{~Hz}, 1 \mathrm{H})$, $8.15(\mathrm{~d}, J=2.0 \mathrm{~Hz}, 1 \mathrm{H}) .{ }^{13} \mathrm{C}$ NMR $\left(\mathrm{CDCl}_{3}, 125 \mathrm{MHz}\right) \delta 13.5\left(\mathrm{CH}_{3}\right), 22.7\left(\mathrm{CH}_{2}\right), 35.8\left(\mathrm{CH}_{2}\right)$, $55.8\left(\mathrm{CH}_{3}\right), 121.3(\mathrm{CH}), 134.7(\mathrm{C}), 134.9(\mathrm{CH}), 142.2(\mathrm{CH}), 155.7(\mathrm{C})$. GC-MS (EI) $183\left([\mathrm{M}]^{+}\right.$, 93\%), 154 (29\%), $141(100 \%), 126(19 \%), 110(9 \%), 98$ (12\%), 70 (7\%). FT-IR (cm ${ }^{-1}$, neat, ATR) $3045(\mathrm{vw}), 2962(\mathrm{w}), 2933(\mathrm{w}), 1569$ (s), 1450 (m), 1409 (s), 1264 (vs), 1222 (m), 1037 (s), $852(\mathrm{~m}), 701$ (s). HRMS (ES+) calcd for $\mathrm{C}_{9} \mathrm{H}_{14} \mathrm{NOS}[\mathrm{M}+\mathrm{H}]^{+}$: 184.0796, found: 184.0790.

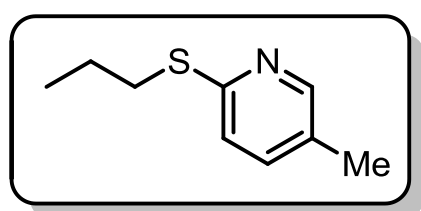

5-Methyl-2-(propylthio)pyridine, ${ }^{\mathbf{2 4}} \mathbf{2 m}(68 \mathrm{mg}, 81 \%)$ was prepared according to the general procedure for thioetherification from 2bromo-5-methylpyridine $(86 \mathrm{mg}, 0.5 \mathrm{mmol})$. The desired thioether was obtained as a clear, colorless oil.

${ }^{1} \mathrm{H} \mathrm{NMR}\left(\mathrm{CDCl}_{3}, 500 \mathrm{MHz}\right) \delta 1.04(\mathrm{t}, J=7.3 \mathrm{~Hz}, 3 \mathrm{H}), 1.68-1.76$ (m, 2H), $2.26(\mathrm{~s}, 3 \mathrm{H}), 3.12(\mathrm{t}, J=7.3 \mathrm{~Hz}, 2 \mathrm{H}), 7.08(\mathrm{~d}, J=8.2 \mathrm{~Hz}, 1 \mathrm{H}), 7.29(\mathrm{dd}, J=8.2,1.8$ $\mathrm{Hz}, 1 \mathrm{H}), 8.26(\mathrm{~s}, 1 \mathrm{H}) .{ }^{13} \mathrm{C}$ NMR $\left(\mathrm{CDCl}_{3}, 125 \mathrm{MHz}\right) \delta 13.8\left(\mathrm{CH}_{3}\right), 18.1\left(\mathrm{CH}_{2}\right), 23.1\left(\mathrm{CH}_{3}\right), 32.6$ $\left(\mathrm{CH}_{2}\right), 122.1(\mathrm{CH}), 128.9(\mathrm{C}), 137.1(\mathrm{CH}), 150.0(\mathrm{CH}), 156.3(\mathrm{C}) . \mathrm{GC}-\mathrm{MS}(\mathrm{EI}) 167\left([\mathrm{M}]^{+}\right.$, 81\%), 152 (94\%), 138 (100\%), 134 (66\%), 125 (99\%), 118 (10\%), 97 (16\%), $92(41 \%), 81$ (47\%), $65(30 \%), 53(11 \%)$. FT-IR ( $\mathrm{cm}^{-1}$, neat, ATR) $2963(\mathrm{w}), 1593(\mathrm{~m}), 1462(\mathrm{vs}), 1365(\mathrm{~m})$, 1111 (vs), 814 (s), 490 (m). HRMS (ES+) calcd for $\mathrm{C}_{9} \mathrm{H}_{14} \mathrm{NS}[\mathrm{M}+\mathrm{H}]^{+}$: 168.0847, found: 168.0847.

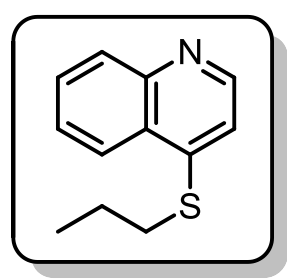

4-(Propylthio)quinoline, $2 \mathrm{n}(72 \mathrm{mg}, 71 \%)$ was prepared according to the general procedure for thioetherification from 4-bromoquinoline (104 mg, 0.5 $\mathrm{mmol})$. The desired thioether was obtained as a clear, yellow oil.

${ }^{1} \mathrm{H}$ NMR $\left(\mathrm{CDCl}_{3}, 500 \mathrm{MHz}\right) \delta 1.12(\mathrm{t}, J=7.3 \mathrm{~Hz}, 3 \mathrm{H}), 1.80-1.88(\mathrm{~m}, 2 \mathrm{H})$, $3.07(\mathrm{t}, J=7.3 \mathrm{~Hz}, 2 \mathrm{H}), 7.16(\mathrm{~d}, J=4.6 \mathrm{~Hz}, 1 \mathrm{H}), 7.54(\mathrm{t}, J=7.6 \mathrm{~Hz}, 1 \mathrm{H})$, $7.70(\mathrm{t}, J=7.6 \mathrm{~Hz}, 1 \mathrm{H}), 8.06(\mathrm{~d}, J=8.5 \mathrm{~Hz}, 1 \mathrm{H}), 8.13(\mathrm{~d}, J=8.5 \mathrm{~Hz}, 1 \mathrm{H})$, $8.70(\mathrm{~d}, J=4.6 \mathrm{~Hz}, 1 \mathrm{H}) .{ }^{13} \mathrm{C}$ NMR $\left(\mathrm{CDCl}_{3}, 125 \mathrm{MHz}\right) \delta 13.9\left(\mathrm{CH}_{3}\right), 22.0\left(\mathrm{CH}_{2}\right), 33.3\left(\mathrm{CH}_{2}\right)$, $116.0(\mathrm{CH}), 123.9(\mathrm{CH}), 126.5(\mathrm{CH}), 126.9(\mathrm{C}), 129.9(\mathrm{CH}), 130.2(\mathrm{CH}), 147.7(\mathrm{C}), 148.2(\mathrm{C})$, 149.5 (CH). GC-MS (EI) 203 ([M] $\left.]^{+}, 63 \%\right), 173$ (18\%), 161 (100\%), 133 (5\%), 117 (15\%), 101 (10\%), 89 (13\%), 75 (7\%), 63 (3\%). FT-IR ( $\mathrm{cm}^{-1}$, neat, ATR) 3061 (vw), $2963(\mathrm{w}), 1561$ (s), 1496 (s), 1375 (m), $1288(\mathrm{~m}), 820$ (s), 755 (vs), 668 (s). HRMS (ES+) calcd for $\mathrm{C}_{12} \mathrm{H}_{14} \mathrm{NS}$ $[\mathrm{M}+\mathrm{H}]^{+}:$204.0847, found: 204.0848 .

\footnotetext{
${ }^{24}$ Bauer, L.; Hirsch, A. L. J. Org. Chem. 1966, 31, 1210.
} 


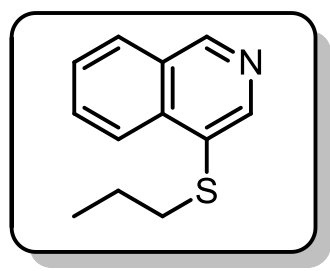

4-(Propylthio)isoquinoline, 20 (78 $\mathrm{mg}, 76 \%)$ was prepared according to the general procedure for thioetherification from 4-bromoisoquinoline (104 $\mathrm{mg}, 0.5 \mathrm{mmol})$. The desired thioether was obtained as a clear, light brown oil.

${ }^{1} \mathrm{H}$ NMR $\left(\mathrm{CDCl}_{3}, 500 \mathrm{MHz}\right) \delta 1.04(\mathrm{t}, J=7.3 \mathrm{~Hz}, 3 \mathrm{H}), 1.64-1.72(\mathrm{~m}$, $2 \mathrm{H}), 2.96(\mathrm{t}, J=7.2 \mathrm{~Hz}, 2 \mathrm{H}), 7.64(\mathrm{tt}, J=7.9,1.0 \mathrm{~Hz}, 1 \mathrm{H}), 7.77(\mathrm{tt}, J=$ $8.0,1.5 \mathrm{~Hz}, 1 \mathrm{H}), 7.98(\mathrm{~d}, J=8.2 \mathrm{~Hz}, 1 \mathrm{H}), 8.34(\mathrm{~d}, J=8.5 \mathrm{~Hz}, 1 \mathrm{H}), 8.57(\mathrm{~s}, 1 \mathrm{H}), 9.12(\mathrm{~s}, 1 \mathrm{H})$. ${ }^{13} \mathrm{C}$ NMR $\left(\mathrm{CDCl}_{3}, 125 \mathrm{MHz}\right) \delta 13.6\left(\mathrm{CH}_{3}\right), 22.9\left(\mathrm{CH}_{2}\right), 36.6\left(\mathrm{CH}_{2}\right), 124.4(\mathrm{CH}), 127.8(\mathrm{CH})$, $128.3(\mathrm{CH}), 128.7(\mathrm{C}), 128.9(\mathrm{C}), 130.9(\mathrm{CH}), 136.1(\mathrm{C}), 144.6(\mathrm{CH}), 151.6(\mathrm{CH}) . \mathrm{GC}-\mathrm{MS}(\mathrm{EI})$ $203\left([\mathrm{M}]^{+}, 97 \%\right), 174(15 \%), 161$ (100\%), 134 (38\%), 117 (21\%), 101 (6\%), 89 (27\%), $75(5 \%)$, $63(5 \%)$. FT-IR ( $\mathrm{cm}^{-1}$, neat, ATR) $3051(\mathrm{vw}), 2961(\mathrm{w}), 1563(\mathrm{w}), 1376(\mathrm{~m}), 1227(\mathrm{~m}), 777(\mathrm{vs})$, 748 (vs), 547 (m). HRMS (ES+) calcd for $\mathrm{C}_{12} \mathrm{H}_{14} \mathrm{NS}[\mathrm{M}+\mathrm{H}]^{+}$: 204.0847, found: 204.0854.

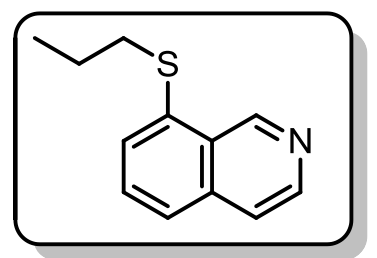

8-(Propylthio)isoquinoline, $2 \mathbf{p}$ ( $73 \mathrm{mg}, 72 \%$ ) was prepared according to the general procedure for thioetherification from 8-bromoisoquinoline $(104 \mathrm{mg}, 0.5 \mathrm{mmol})$. The desired thioether was obtained as a clear, yellow oil.

${ }^{1} \mathrm{H}$ NMR $\left(\mathrm{CDCl}_{3}, 500 \mathrm{MHz}\right) \delta 1.06(\mathrm{t}, J=7.3 \mathrm{~Hz}, 3 \mathrm{H}), 1.68-1.76(\mathrm{~m}$, $2 \mathrm{H}), 3.02(\mathrm{t}, J=7.3 \mathrm{~Hz}, 2 \mathrm{H}), 7.56-7.67(\mathrm{~m}, 4 \mathrm{H}), 8.56(\mathrm{~d}, J=5.8 \mathrm{~Hz}$,

$1 \mathrm{H}), 9.78(\mathrm{~s}, 1 \mathrm{H}) .{ }^{13} \mathrm{C}$ NMR $\left(\mathrm{CDCl}_{3}, 125 \mathrm{MHz}\right) \delta 13.7\left(\mathrm{CH}_{3}\right), 22.7\left(\mathrm{CH}_{2}\right), 36.4\left(\mathrm{CH}_{2}\right), 120.9$ $(\mathrm{CH}), 125.1(\mathrm{CH}), 128.0(\mathrm{C}), 128.2(\mathrm{CH}), 130.2(\mathrm{CH}), 136.3(\mathrm{C}), 136.9(\mathrm{C}), 143.7(\mathrm{CH}), 150.2$ (CH). GC-MS (EI) 203 ([M] $\left.{ }^{+}, 83 \%\right), 173$ (16\%), 161 (100\%), 134 (17\%), 128 (8\%), $117(24 \%)$, $89(12 \%), 75(5 \%), 63(4 \%)$. FT-IR $\left(\mathrm{cm}^{-1}\right.$, neat, ATR) $3049(\mathrm{vw}), 2962(\mathrm{w}), 1611(\mathrm{w}), 1552(\mathrm{~s})$, 1382 (w), 1265 (m), 1205 (m), 977 (w), 829 (vs), 795 (w), 746 (s), 642 (s). HRMS (CI+) calcd for $\mathrm{C}_{12} \mathrm{H}_{14} \mathrm{NS}[\mathrm{M}+\mathrm{H}]^{+}: 204.0847$, found: 204.0842 .

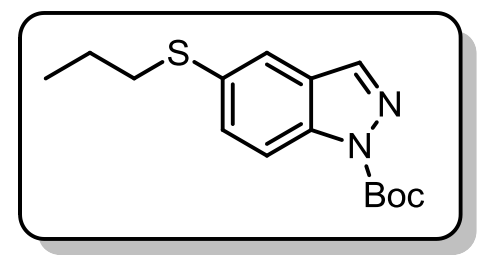

tert-Butyl 5-(Propylthio)-1H-indazole-1-carboxylate, 2q (130 $\mathrm{mg}, 89 \%$ ) was prepared according to the general procedure for thioetherification from tert-butyl 5-bromo-1H-indazole-1carboxylate (149 $\mathrm{mg}, 0.5 \mathrm{mmol})$. The desired thioether was obtained as a clear, light brown oil.

${ }^{1} \mathbf{H}$ NMR $\left(\mathrm{CDCl}_{3}, 500 \mathrm{MHz}\right) \delta 1.06(\mathrm{t}, J=7.34 \mathrm{~Hz}, 3 \mathrm{H}), 1.71-$ $1.79(\mathrm{~m}, 2 \mathrm{H}), 1.73(\mathrm{~s}, 9 \mathrm{H}), 3.02(\mathrm{t}, J=7.34 \mathrm{~Hz}, 2 \mathrm{H}), 7.23(\mathrm{dd}, J=8.31,0.98 \mathrm{~Hz}, 1 \mathrm{H}), 7.58(\mathrm{~d}, J$ $=8.56 \mathrm{~Hz}, 1 \mathrm{H}), 8.08(\mathrm{~s}, 1 \mathrm{H}), 8.10(\mathrm{~s}, 1 \mathrm{H}),{ }^{13} \mathrm{C} \mathrm{NMR}\left(\mathrm{CDCl}_{3}, 125 \mathrm{MHz}\right) \delta 13.7\left(\mathrm{CH}_{3}\right), 22.4$ $\left(\mathrm{CH}_{2}\right), 28.4\left(\mathrm{CH}_{3}\right), 35.3\left(\mathrm{CH}_{2}\right), 85.1(\mathrm{C}), 112.7(\mathrm{CH}), 121.1(\mathrm{CH}), 123.9(\mathrm{C}), 124.4(\mathrm{CH}), 139.5$ (CH), 140.1 (C), 140.6 (C), $149.4(\mathrm{C})$. FT-IR ( $\mathrm{cm}^{-1}$, neat, ATR) $2963(\mathrm{w}), 1756$ (m), 1733 (s), 1406 (s), 1369 (s), 1292 (s), 1147 (vs), 1027 (s), 919 (s), 846 (m) 619 (w). HRMS (ES+) calcd for $\mathrm{C}_{15} \mathrm{H}_{21} \mathrm{~N}_{2} \mathrm{O}_{2} \mathrm{~S}[\mathrm{M}+\mathrm{H}]^{+}:$293.1324, found: 293.1323 .

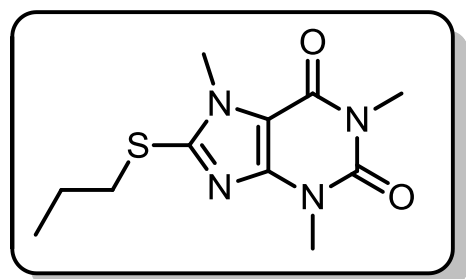

1,3,7-Trimethyl-8-(propylthio)-1H-purine-2,6(3H,7H),-dione, ${ }^{25}$ 2r (94 $\mathrm{mg}, 70 \%)$ was prepared according to the general procedure for thioetherification from 8-bromocaffeine (136 $\mathrm{mg}, 0.5 \mathrm{mmol})$. The desired thioether was obtained as an off white powder $(\mathrm{mp}=$ $\left.124^{\circ} \mathrm{C}\right)$.

\footnotetext{
${ }^{25}$ He, Z.; Luo, F.; Li, Y.; Zhu, G. Tetrahedron Lett., 2013, 54, 5907.
} 
${ }^{1} \mathrm{H}$ NMR $\left(\mathrm{CDCl}_{3}, 500 \mathrm{MHz}\right) \delta 1.00(\mathrm{t}, J=7.3 \mathrm{~Hz}, 3 \mathrm{H}), 1.70-1.78(\mathrm{~m}, 2 \mathrm{H}), 3.20(\mathrm{t}, J=7.2 \mathrm{~Hz}$, 2H), $3.32(\mathrm{~s}, 3 \mathrm{H}), 3.50(\mathrm{~s}, 3 \mathrm{H}), 3.79(\mathrm{~s}, 3 \mathrm{H}) .{ }^{13} \mathrm{C} \mathbf{N M R}\left(\mathrm{CDCl}_{3}, 125 \mathrm{MHz}\right) \delta 13.4\left(\mathrm{CH}_{3}\right), 23.2$ $\left(\mathrm{CH}_{2}\right), 28.0\left(\mathrm{CH}_{3}\right), 29.8\left(\mathrm{CH}_{3}\right), 32.3\left(\mathrm{CH}_{3}\right), 34.9\left(\mathrm{CH}_{2}\right), 108.6(\mathrm{C}), 148.7(\mathrm{C}), 151.5(\mathrm{C}), 151.7$ (C), 154.7 (C). FT-IR ( $\mathrm{cm}^{-1}$, neat, ATR) 2961 (vw), 1690 (s), 1645 (vs), 1532 (m), 1451 (m), 1364 (m), 1282 (w), 1034 (w), 746 (s), 493 (m). HRMS (ES+) calcd for $\mathrm{C}_{11} \mathrm{H}_{17} \mathrm{~N}_{4} \mathrm{O}_{2} \mathrm{~S}[\mathrm{M}+\mathrm{H}]^{+}$: 269.1072, found: 269.1075 . 


\section{General Procedure for Thioetherification Using Thiols and 1b}

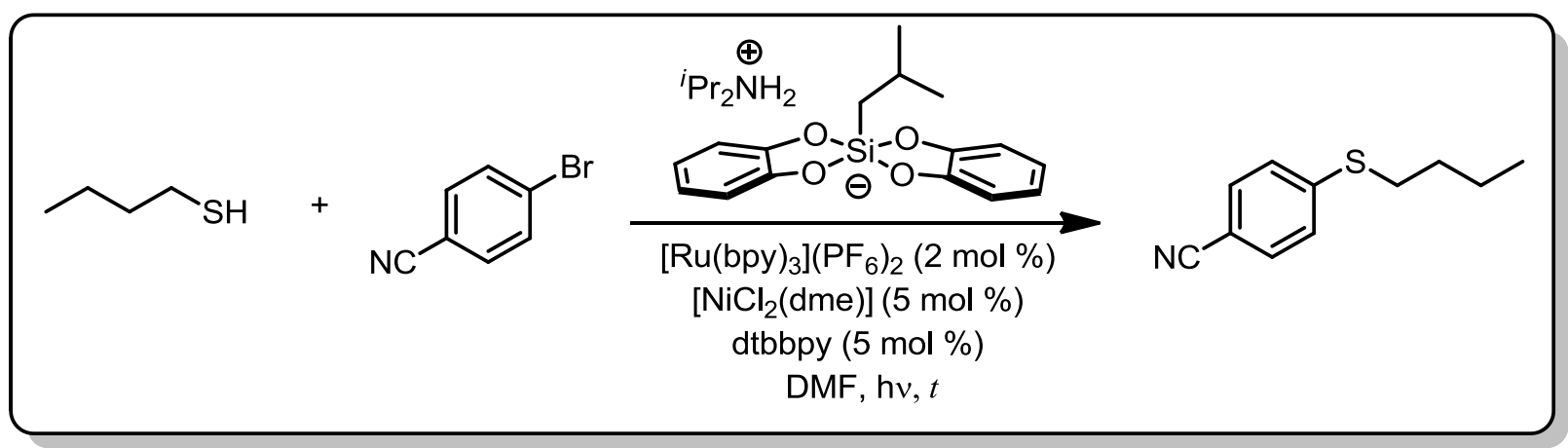

\section{4-(Butylthio)benzonitrile ${ }^{26}(3 a)$}

To an $8 \mathrm{~mL}$ reaction vial equipped with an appropriately sized stir bar were added dtbbpy (6.7 $\mathrm{mg}, 0.025,0.05$ equiv) and $\left[\mathrm{NiCl}_{2}(\mathrm{dme})\right](5.5 \mathrm{mg}, 0.025 \mathrm{mmol}, 0.05$ equiv). The vial was sealed with a cap containing a TFE lined silicone septa and placed under a $\mathrm{N}_{2}$ atmosphere via an inlet needle. The vial was charged with $\sim 1.5 \mathrm{~mL}$ of anhyd THF and the resulting suspension was heated briefly with a heat gun until a homogenous pale green solution was observed. The solution was cooled in an ice water bath, resulting in the immediate precipitation of an evergreen solid. The solution was then evaporated in vacuo to give the ligated nickel complex. ${ }^{27}$ The cap was removed from the vial and alkylsilicate 1 b $(303 \mathrm{mg}, 0.75 \mathrm{mmol}, 1.5$ equiv), 4bromobenzonitrile (91 mg, $0.50 \mathrm{mmol}, 1$ equiv), ${ }^{28}$ and $\left[\mathrm{Ru}(\mathrm{bpy})_{3}\right]\left(\mathrm{PF}_{6}\right)(8.6 \mathrm{mg}, 0.01 \mathrm{mmol}, 0.02$ equiv) were added. The vial was sealed with a cap containing a TFE lined silicone septa and was evacuated three times via an inlet needle then purged with argon. The vial was then charged with a solution of 1-butanethiol $(64.6 \mu \mathrm{L}, 54 \mathrm{mg}, 0.60 \mathrm{mmol}, 1.2$ equiv) dissolved in anhyd, degassed DMF $(5 \mathrm{~mL})$ via a syringe. The cap was sealed with Parafilm ${ }^{\circledR}$, and the now bright red solution was irradiated in the aforementioned LED reactor. The temperature of the reaction was maintained at approximately $27{ }^{\circ} \mathrm{C}$ via a fan. The solution was stirred vigorously while being irradiated. Reaction progress was monitored by HPLC and/or GC/MS. Once judged to be complete, the now opaque, milky-brown solution was transferred to a separatory funnel and diluted with saturated aqueous $\mathrm{Na}_{2} \mathrm{CO}_{3}(20 \mathrm{~mL})^{29}$ and $\mathrm{Et}_{2} \mathrm{O}^{30}(\sim 20 \mathrm{~mL})$. The layers were separated, ${ }^{31}$ and the aqueous layer was extracted with $\mathrm{Et}_{2} \mathrm{O}(3 \times \sim 20 \mathrm{~mL})$. The combined organic layers were washed with saturated aqueous $\mathrm{Na}_{2} \mathrm{CO}_{3}(2 \times \sim 30 \mathrm{~mL})$, deionized $\mathrm{H}_{2} \mathrm{O}(\sim 30 \mathrm{~mL})$, and brine $(\sim 50 \mathrm{~mL}){ }^{32}$ The combined organic layer were dried $\left(\mathrm{MgSO}_{4}\right)$ and the solvent was removed

\footnotetext{
${ }^{26}$ Qiao, Z.; Wei, J.; Jiang, X. Org. Lett. 2014 , 16, 1212.

${ }^{27}$ We have performed the reaction with and without pre-ligation of the ligand. We found that there is no effect on the yield of cross couplings performed here. However, it was necessary for certain systems in our previous report, see ref 3 .

${ }^{28}$ Liquid bromides were added later as a solution in DMF

${ }^{29}$ Alternatively $2 \mathrm{M} \mathrm{NaOH}$ may be used

${ }^{30}$, EtOAc was used in place of $\mathrm{Et}_{2} \mathrm{O}$ for the extractions when isolating products containing polar functional groups.

${ }^{31}$ Note that a precipitate will often form and rest at the interface between the organic and aqueous layers. It can be discarded during the washes without compromising yield.

${ }^{32}$ In certain cases, specifically when working with systems that did not contain basic moieties, an optionally four wash can be added. Following the saturated aqueous $\mathrm{Na}_{2} \mathrm{CO}_{3}$ (or $2 \mathrm{M} \mathrm{NaOH}$ ) wash, a $2 \mathrm{M} \mathrm{HCl}(\sim 30 \mathrm{~mL}) \mathrm{may} \mathrm{be}$ used. This will remove any residual dtbbpy.
} 
in vacuo by rotary evaporation. Further purification was accomplished by $\mathrm{SiO}_{2}$ column chromatography (gradient hexane/EtOAc). to give the desired thioether, 3a, as a colorless oil (94 mg, 98\%).

${ }^{1} \mathrm{H}$ NMR $\left(\mathrm{CDCl}_{3}, 500 \mathrm{MHz}\right) \delta 0.94(\mathrm{t}, J=7.3 \mathrm{~Hz}, 3 \mathrm{H}), 1.43-1.51(\mathrm{~m}, 2 \mathrm{H}), 1.63-1.71(\mathrm{~m}, 2 \mathrm{H})$, $2.97(\mathrm{t}, J=7.3 \mathrm{~Hz}, 2 \mathrm{H}), 7.28(\mathrm{~d}, J=8.6 \mathrm{~Hz}, 2 \mathrm{H}), 7.51(\mathrm{~d}, J=8.6 \mathrm{~Hz}, 2 \mathrm{H}) .{ }^{13} \mathrm{C} \mathrm{NMR}\left(\mathrm{CDCl}_{3}\right.$, $125 \mathrm{MHz}) \delta 13.8\left(\mathrm{CH}_{3}\right), 22.2\left(\mathrm{CH}_{2}\right), 30.9\left(\mathrm{CH}_{2}\right), 31.8\left(\mathrm{CH}_{2}\right), 108.2(\mathrm{C}), 119.2(\mathrm{C}), 126.9(\mathrm{CH})$, $132.4(\mathrm{CH}), 145.6(\mathrm{C})$. GC-MS (EI) 191 ([M] $\left.{ }^{+}, 80 \%\right), 148$ (33\%), $135(100 \%), 104(8 \%), 90$ (10\%), $63(6 \%), 57(19 \%)$. FT-IR $\left(\mathrm{cm}^{-1}\right.$, neat, ATR) $2958(\mathrm{~m}), 2931(\mathrm{~m}), 2225(\mathrm{~s}), 1592(\mathrm{~s})$, 1485 (s), 1088 (vs), 818 (vs), 542 (vs). HRMS (CI+) calcd for $\mathrm{C}_{11} \mathrm{H}_{13} \mathrm{NS}[\mathrm{M}]^{+}:$191.0769, found: 191.0768 .

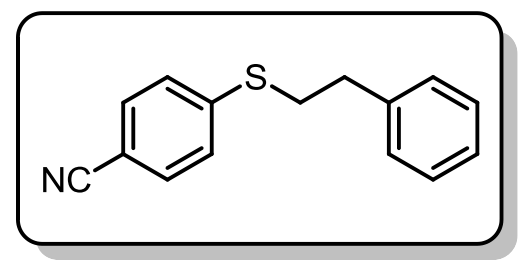

4-(Phenethylthio)benzonitrile, ${ }^{33}$ 3b $(115 \mathrm{mg}$, 96\%) was prepared according to the general procedure for thioetherification from 4-bromobenzonitrile $(91 \mathrm{mg}, 0.50 \mathrm{mmol})$ and 2-phenylethanethiol $(79.6 \mu \mathrm{L}, 82 \mathrm{mg}, 0.60 \mathrm{mmol})$. The desired thioether was obtained as a clear, light brown oil.

${ }^{1} \mathrm{H} \mathrm{NMR}\left(\mathrm{CDCl}_{3}, 500 \mathrm{MHz}\right) \delta 3.00(\mathrm{t}, J=7.7 \mathrm{~Hz}, 2 \mathrm{H}), 3.25(\mathrm{t}$, $J=7.7 \mathrm{~Hz}, 2 \mathrm{H}), 7.21-7.29(\mathrm{~m}, 3 \mathrm{H}), 7.30-7.37(\mathrm{~m}, 4 \mathrm{H}), 7.54(\mathrm{~d}, J=8.1 \mathrm{~Hz}, 2 \mathrm{H}) .{ }^{13} \mathrm{C}$ NMR $\left(\mathrm{CDCl}_{3}, 125 \mathrm{MHz}\right) \delta 33.7\left(\mathrm{CH}_{2}\right), 35.3\left(\mathrm{CH}_{2}\right), 108.5(\mathrm{C}), 119.1(\mathrm{C}), 127.1(\mathrm{CH}), 127.2(\mathrm{CH})$, $128.7(\mathrm{CH}), 128.9(\mathrm{CH}), 132.5(\mathrm{CH}), 139.6(\mathrm{C}), 144.8(\mathrm{C})$. GC-MS (EI) $239\left([\mathrm{M}]^{+}, 100 \%\right), 146$ (76\%), 105 (92\%), 91 (74\%), $79(10 \%), 77(20 \%), 65(9 \%)$. FT-IR $\left(\mathrm{cm}^{-1}\right.$, neat, ATR) $3028(\mathrm{vw})$, 2926 (vw), 2224 (s), 1592 (s), 1485 (s), 1087 (vs), 817 (vs), 697 (vs), 542 (vs). HRMS (CI+) calcd for $\mathrm{C}_{15} \mathrm{H}_{14} \mathrm{NS}[\mathrm{M}+\mathrm{H}]^{+}: 240.0847$, found: 240.0839 .

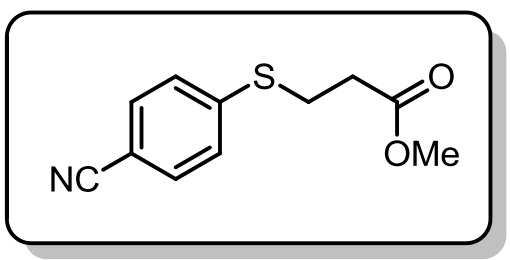

Methyl 3-((4-Cyanophenyl)thio)propanoate, 3c (95 mg, 86\%) was prepared according to the general procedure for thioetherification from 4-bromobenzonitrile $(91 \mathrm{mg}, 0.50 \mathrm{mmol})$ and methyl 3-mercaptopropanoate $(66.6 \mu \mathrm{L}, 72 \mathrm{mg}, 0.60 \mathrm{mmol})$. The desired thioether was obtained as a white solid $\left(\mathrm{mp}=46^{\circ} \mathrm{C}\right)$. ${ }^{1} \mathrm{H}$ NMR $\left(\mathrm{CDCl}_{3}, 500 \mathrm{MHz}\right) \delta 2.69(\mathrm{t}, J=7.3 \mathrm{~Hz}, 2 \mathrm{H}), 3.25(\mathrm{t}, J$ $=7.3 \mathrm{~Hz}, 2 \mathrm{H}), 3.71(\mathrm{~s}, 3 \mathrm{H}), 7.33(\mathrm{~d}, J=8.4 \mathrm{~Hz}, 2 \mathrm{H}), 7.54(\mathrm{~d}, J=8.3 \mathrm{~Hz}, 2 \mathrm{H}) .{ }^{13} \mathrm{C}$ NMR $\left(\mathrm{CDCl}_{3}, 125 \mathrm{MHz}\right) \delta 27.4\left(\mathrm{CH}_{2}\right), 33.8\left(\mathrm{CH}_{2}\right), 52.3\left(\mathrm{CH}_{3}\right), 109.1(\mathrm{C}), 118.9(\mathrm{C}), 127.7(\mathrm{CH})$, $132.7(\mathrm{CH}), 143.8(\mathrm{C}), 171.9$ (C). GC-MS (EI) $221\left([\mathrm{M}]^{+}, 100 \%\right), 161(67 \%), 148(45 \%) 134$ (27\%), 87 (13\%), 59 (16\%). FT-IR ( $\mathrm{cm}^{-1}$, neat, ATR) $2951(\mathrm{vw}), 2226(\mathrm{~m}), 1732(\mathrm{vs}), 1593(\mathrm{~m})$, 1174 (s), 1087 (s), 820 (s), 543 (s). HRMS (CI+) calcd for $\mathrm{C}_{11} \mathrm{H}_{12} \mathrm{NO}_{2} \mathrm{~S}[\mathrm{M}+\mathrm{H}]^{+}:$222.0589, found: 222.0591 .

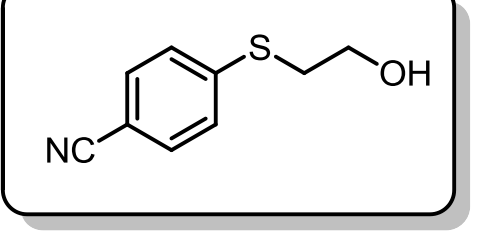

4-((2-Hydroxyethyl)thio)benzonitrile, ${ }^{\mathbf{3 4}} \mathbf{3 d}(64 \mathrm{mg}, 71 \%)$ was prepared according to the general procedure for thioetherification from 4-bromobenzonitrile (91 $\mathrm{mg}, 0.50 \mathrm{mmol})$ and 2mercaptoethanol $(35.8 \mu \mathrm{L}, 31 \mathrm{mg}, 0.60 \mathrm{mmol})$. The desired thioether was obtained as a yellow solid $\left(\mathrm{mp}=52^{\circ} \mathrm{C}\right)$.

${ }^{1} \mathrm{H}$ NMR $\left(\mathrm{CDCl}_{3}, 500 \mathrm{MHz}\right) \delta 2.29($ br s, $1 \mathrm{H}), 3.18(\mathrm{t}, J=5.9 \mathrm{~Hz}$,

\footnotetext{
${ }^{33}$ Van Der Walt, M. M.; Terre'Blanche, G.; Lourens, A. C. U.; Petzer, A.; Petzer, J. P. Bioorg. Med. Chem. Lett., $2012,22,7367$.

${ }^{34}$ Klenke, B. et al. WO 2013110643, 2013.
} 
2H), $3.83(\mathrm{t}, J=5.9 \mathrm{~Hz}, 2 \mathrm{H}), 7.35(\mathrm{~d}, J=8.3 \mathrm{~Hz}, 2 \mathrm{H}), 7.51(\mathrm{~d}, J=8.1 \mathrm{~Hz}, 2 \mathrm{H}) .{ }^{13} \mathrm{C}$ NMR $\left(\mathrm{CDCl}_{3}, 125 \mathrm{MHz}\right) \delta 35.2\left(\mathrm{CH}_{2}\right), 60.7\left(\mathrm{CH}_{2}\right), 108.8(\mathrm{C}), 118.9(\mathrm{C}), 127.7(\mathrm{CH}), 132.5(\mathrm{CH})$, 143.9 (C). GC-MS (EI) 179 ([M] $\left.]^{+}, 76 \%\right), 177$ (7\%), 162 (12\%), 148 (100\%), 135 (60\%), 121 (26\%), 104 (23\%), $91(31 \%), 75(31 \%) 63$ (18\%). FT-IR ( $\mathrm{cm}^{-1}$, neat, ATR) 3403 (m, br), 2930 (w), 2878 (w), 2225 (s), 1592 (s), 1486 (s), 1087 (s), 1042 (s), 817 (vs), 542 (vs). HRMS (ES+) calcd for $\mathrm{C}_{9} \mathrm{H}_{9} \mathrm{NOSNa}[\mathrm{M}+\mathrm{Na}]^{+}: 202.0303$, found: 202.0304 .

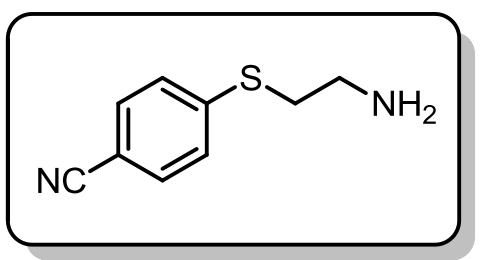

4-((2-Aminoethyl)thio)benzonitrile, $3 \mathbf{e} \quad(81 \mathrm{mg}, \quad 91 \%)$ was prepared according to the general procedure for thioetherification from 4-bromobenzonitrile $(91 \mathrm{mg}, 0.50 \mathrm{mmol})$ and cysteamine hydrochloride $(68 \mathrm{mg}, 0.60 \mathrm{mmol})$. The desired thioether was obtained as a clear, yellow oil.

${ }^{1} \mathrm{H} \mathrm{NMR}\left(\mathrm{CDCl}_{3}, 500 \mathrm{MHz}\right) \delta 1.96(\mathrm{br} \mathrm{s}, 2 \mathrm{H}), 2.96(\mathrm{t}, J=6.4 \mathrm{~Hz}$, $2 \mathrm{H}), 3.07(\mathrm{t}, J=6.4 \mathrm{~Hz}, 2 \mathrm{H}), 7.31(\mathrm{~d}, J=8.6 \mathrm{~Hz}, 2 \mathrm{H}), 7.49(\mathrm{~d}, J=8.3 \mathrm{~Hz}, 2 \mathrm{H}) .{ }^{13} \mathrm{C} \mathrm{NMR}$ $\left(\mathrm{CDCl}_{3}, 125 \mathrm{MHz}\right) \delta 36.1\left(\mathrm{CH}_{2}\right), 40.8\left(\mathrm{CH}_{2}\right), 108.6(\mathrm{C}), 119.0(\mathrm{C}), 127.5(\mathrm{CH}), 132.5(\mathrm{CH})$, 144.2 (C). GC-MS (EI) $178\left([\mathrm{M}]^{+}, 52 \%\right), 149$ (100\%), 134 (17\%), $116(8 \%), 104$ (9\%), 90 (8\%), $76(9 \%) 63$ (7\%). FT-IR (cm ${ }^{-1}$, neat, ATR) 3364 (vw, br), 2926 (w), 2224 (s), 1591 (vs), 1485 (s), 1087 (vs), 817 (vs), 543 (vs). HRMS (ES+) calcd for $\mathrm{C}_{9} \mathrm{H}_{11} \mathrm{~N}_{2} \mathrm{~S}[\mathrm{M}+\mathrm{H}]^{+}:$179.0643, found: 179.0635 .

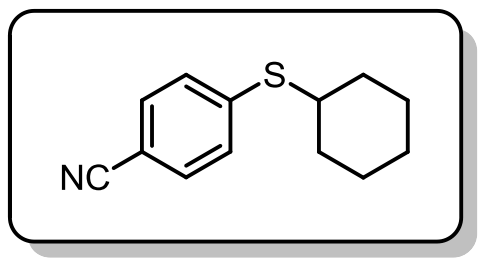

4-(Cyclohexylthio)benzonitrile, ${ }^{35}$ 3f $(104 \quad \mathrm{mg}, \quad 96 \%)$ was prepared according to the general procedure for thioetherification from 4-bromobenzonitrile (91 $\mathrm{mg}, \quad 0.50 \mathrm{mmol})$ and cyclohexanethiol $(73.4 \mu \mathrm{L}, 70 \mathrm{mg}, 0.60 \mathrm{mmol})$. The desired thioether was obtained as a light yellow oil.

${ }^{1} \mathrm{H}$ NMR $\left(\mathrm{CDCl}_{3}, 500 \mathrm{MHz}\right) \delta 1.24-1.50(\mathrm{~m}, 5 \mathrm{H}), 1.61-1.71$ $(\mathrm{m}, 1 \mathrm{H}), 1.76-1.86(\mathrm{~m}, 2 \mathrm{H}), 1.98-2.12(\mathrm{~m}, 2 \mathrm{H}), 3.30(\mathrm{tt}, J=10.3,3.7 \mathrm{~Hz}, 1 \mathrm{H}), 7.34(\mathrm{~d}, J=8.3$ $\mathrm{Hz}, 2 \mathrm{H}), 7.52(\mathrm{~d}, J=8.3 \mathrm{~Hz}, 2 \mathrm{H}) .{ }^{13} \mathrm{C} \mathbf{N M R}\left(\mathrm{CDCl}_{3}, 125 \mathrm{MHz}\right) \delta 25.8\left(\mathrm{CH}_{2}\right), 26.1\left(\mathrm{CH}_{2}\right), 33.1$ $\left(\mathrm{CH}_{2}\right), 45.1(\mathrm{CH}), 108.7(\mathrm{C}), 119.1(\mathrm{C}), 128.8(\mathrm{CH}), 132.4(\mathrm{CH}), 144.2(\mathrm{CH})$. GC-MS (EI) 217 $\left([\mathrm{M}]^{+}, 91 \%\right), 135(100 \%), 90(10 \%), 83(81 \%), 67(24 \%), 55(79 \%)$. FT-IR $\left(\mathrm{cm}^{-1}\right.$, neat, ATR) 2929 (s), 2853 (m), 2225 (s), 1592 (s), 1485 (s), 1087 (vs), 818 (vs), 543 (vs). HRMS (ES+) calcd for $\mathrm{C}_{13} \mathrm{H}_{15} \mathrm{NS}[\mathrm{M}]^{+}: 217.0925$, found: 217.0927 .

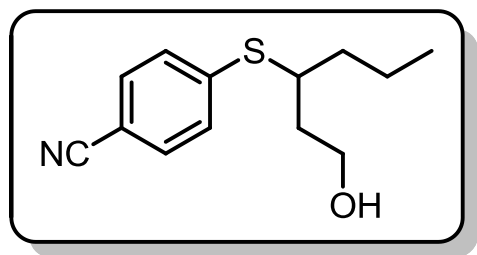

4-((1-Hydroxyhexan-3-yl)thio)benzonitrile, $3 g$ (114 mg, 97\%) was prepared according to the general procedure for thioetherification from 4-bromobenzonitrile $(91 \mathrm{mg}, 0.50 \mathrm{mmol})$ and 3-mercaptohexan-1-ol $(83.0 \mu \mathrm{L}, 80 \mathrm{mg}, 0.60 \mathrm{mmol})$. The desired thioether was obtained as a clear, pale yellow oil.

${ }^{1} \mathrm{H}$ NMR $\left(\mathrm{CDCl}_{3}, 500 \mathrm{MHz}\right) \delta 0.91(\mathrm{t}, J=7.2 \mathrm{~Hz}, 3 \mathrm{H}), 1.41-$ $1.56(\mathrm{~m}, 2 \mathrm{H}), 1.57-1.72(\mathrm{~m}, 3 \mathrm{H}), 1.82(\mathrm{ddt}, J=14.2,8.3,5.7 \mathrm{~Hz}, 1 \mathrm{H}), 1.94(\mathrm{ddt}, J=14.3,8.3$, $5.7 \mathrm{~Hz} 1 \mathrm{H}), 3.46-3.52(\mathrm{~m}, 1 \mathrm{H}), 3.80(\mathrm{qd}, J=11.3,6.4 \mathrm{~Hz}, 2 \mathrm{H}), 7.39(\mathrm{~d}, J=8.2 \mathrm{~Hz}, 2 \mathrm{H}), 7.51(\mathrm{~d}$, $J=8.2 \mathrm{~Hz}, 2 \mathrm{H}) .{ }^{13} \mathrm{C} \mathbf{N M R}\left(\mathrm{CDCl}_{3}, 125 \mathrm{MHz}\right) \delta 14.2\left(\mathrm{CH}_{3}\right), 20.2\left(\mathrm{CH}_{2}\right), 37.4\left(\mathrm{CH}_{2}\right), 37.6$ $\left(\mathrm{CH}_{2}\right), 44.4(\mathrm{CH}), 60.3\left(\mathrm{CH}_{2}\right), 108.8(\mathrm{C}), 119.1(\mathrm{C}), 129.0(\mathrm{CH}), 132.5(\mathrm{CH}), 144.7(\mathrm{C})$. GC-MS

\footnotetext{
${ }^{35}$ Fernandez-Rodriguez, M. A.; Shen, Q.; Hartwig, J. F. Chem.-Eur. J. 2006, 12, 7782.
} 
(EI) $235\left([\mathrm{M}]^{+}, 4 \%\right), 207(17 \%), 135(10 \%), 125(100 \%), 83(15 \%), 55(24 \%)$. FT-IR $\left(\mathrm{cm}^{-1}\right.$, neat, ATR) 3434 (m, br), 2957 (m), 2931 (m), 2226 (s), 1591 (vs), 1485 (s), 1086 (vs), 1048 (s), 820 (vs), 543 (vs). HRMS (ES+) calcd for $\mathrm{C}_{13} \mathrm{H}_{18} \mathrm{NOS}[\mathrm{M}+\mathrm{H}]^{+}:$236.1109, found: 236.1105 .

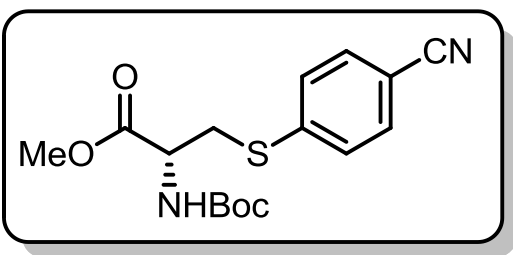

(R)-Methyl 2-((tert-Butoxycarbonyl)amino)-3-((4cyanophenyl)thio)propanoate, $3 \mathrm{~h}$ (140 $\mathrm{mg}, 83 \%)$ was prepared according to the general procedure for thioetherification from 4-bromobenzonitrile (91 $\mathrm{mg}, 0.50$ mmol) and (R)-methyl 2-((tert-butoxycarbonyl)amino)-3mercaptopropanoate $(141 \mathrm{mg}, 0.60 \mathrm{mmol})$. The desired thioether was obtained as a white solid $\left(\mathrm{mp}=57^{\circ} \mathrm{C}\right) .[\alpha]^{23}{ }_{\mathrm{D}}=+28.2\left(\mathrm{CHCl}_{3}, c=1.00\right)$.

${ }^{1} \mathrm{H} \mathrm{NMR}\left(\mathrm{CDCl}_{3}, 500 \mathrm{MHz}\right) \delta 1.41(\mathrm{~s}, 9 \mathrm{H}), 3.40(\mathrm{dd}, J=13.7,4.7 \mathrm{~Hz}, 1 \mathrm{H}), 3.51(\mathrm{dd}, J=13.9$, $4.7 \mathrm{~Hz}, 1 \mathrm{H}), 3.67(\mathrm{~s}, 3 \mathrm{H}), 4.37-4.70(\mathrm{~m}, 1 \mathrm{H}), 5.01-5.51(\mathrm{~m}, 1 \mathrm{H}), 7.40(\mathrm{~d}, J=8.3 \mathrm{~Hz}, 2 \mathrm{H}), 7.53$ $(\mathrm{d}, J=8.6 \mathrm{~Hz}, 2 \mathrm{H}) .{ }^{13} \mathrm{C} \mathrm{NMR}\left(\mathrm{CDCl}_{3}, 125 \mathrm{MHz}\right) \delta 28.5\left(\mathrm{CH}_{3}\right), 35.4\left(\mathrm{CH}_{2}\right), 52.9\left(\mathrm{CH}_{3}\right), 53.4$ $(\mathrm{CH}), 80.7(\mathrm{C}), 109.5(\mathrm{C}), 118.8(\mathrm{C}), 128.6(\mathrm{CH}), 132.5(\mathrm{CH}), 143.2(\mathrm{C}), 155.1(\mathrm{C}), 170.8(\mathrm{C})$. FT-IR (cm ${ }^{-1}$, neat, ATR) 3362 (w, br), 2964 (w), 2227 (w), 1740 (m), 1709 (s), 1487 (m), 1367 (m), 1244 (s), 1160 (vs), 1049 (m), 823 (m), 544 (m). HRMS (ES+) calcd for $\mathrm{C}_{16} \mathrm{H}_{21} \mathrm{~N}_{2} \mathrm{O}_{4} \mathrm{~S}$ $[\mathrm{M}+\mathrm{H}]^{+}:$337.1222, found: 337.1221 .

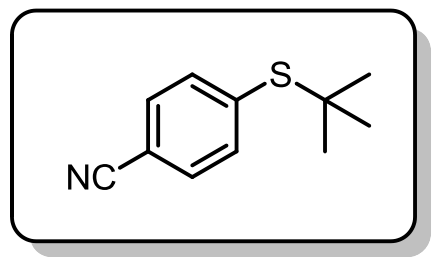

(4-(tert-Butylthio)benzonitrile, 3i (94 mg, 98\%) was prepared according to the general procedure for thioetherification from 4bromobenzonitrile $(91 \mathrm{mg}, 0.50 \mathrm{mmol})$ and tert-butylthiol $(67.6 \mu \mathrm{L}$, $54 \mathrm{mg}, 0.60 \mathrm{mmol})$. The desired thioether was obtained as an off white solid $\left(\mathrm{mp}=51^{\circ} \mathrm{C}\right)$.

${ }^{1} \mathrm{H}$ NMR $\left(\mathrm{CDCl}_{3}, 500 \mathrm{MHz}\right) \delta 1.31(\mathrm{~s}, 9 \mathrm{H}), 7.59(\mathrm{~d}, J=8.3 \mathrm{~Hz}$, 2H), $7.61(\mathrm{~d}, J=8.3 \mathrm{~Hz}, 2 \mathrm{H}) .{ }^{13} \mathrm{C} \mathrm{NMR}\left(\mathrm{CDCl}_{3}, 125 \mathrm{MHz}\right) \delta 31.3$ $\left(\mathrm{CH}_{3}\right), 47.5(\mathrm{C}), 112.4(\mathrm{C}), 118.7(\mathrm{C}), 132.1(\mathrm{CH}), 137.4(\mathrm{CH}), 140.1(\mathrm{C})$. GC-MS (EI) 191 ([M] $\left.]^{+}, 38 \%\right), 176(7 \%), 135(73 \%), 107$ (6\%), 90 (13\%), 69 (6\%), 57 (100\%). FT-IR (cm ${ }^{-1}$, neat, ATR) 3051 (vw), 2959 (m), 2231(m), 1483 (m), 1366 (m), 1163 (m), 834 (s), 551 (vs). HRMS (CI+) calcd for $\mathrm{C}_{11} \mathrm{H}_{14} \mathrm{NS}[\mathrm{M}+\mathrm{H}]^{+}:$192.0847, found: 192.0845 .

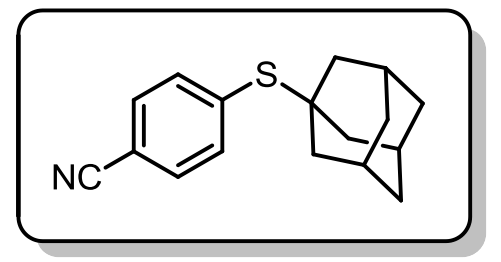

4-(Adamantan-1-ylthio)benzonitrile, ${ }^{\mathbf{3 6}} \mathbf{3 j}$ (119 $\left.\mathrm{mg}, 88 \%\right)$ was prepared according to the general procedure for thioetherification from 4-bromobenzonitrile $(91 \mathrm{mg}, 0.50 \mathrm{mmol})$ and 1adamantanethiol $(101 \mathrm{mg}, 0.60 \mathrm{mmol})$. The desired thioether was obtained as a white solid $\left(\mathrm{mp}=137^{\circ} \mathrm{C}\right)$.

${ }^{1} \mathrm{H} \mathrm{NMR}\left(\mathrm{CDCl}_{3}, 500 \mathrm{MHz}\right) \delta 1.62(\mathrm{~d}, J=12.0 \mathrm{~Hz}, 3 \mathrm{H}), 1.68(\mathrm{~d}, J$ $=12.0 \mathrm{~Hz}, 3 \mathrm{H}), 1.84$ (br s, 6H), 2.05 (br s, 3H), 7.60 (br s, 4H). ${ }^{13} \mathrm{C} \mathrm{NMR}\left(\mathrm{CDCl}_{3}, 125 \mathrm{MHz}\right)$ $\delta 30.2(\mathrm{CH}), 36.2\left(\mathrm{CH}_{2}\right), 44.0\left(\mathrm{CH}_{2}\right), 49.8(\mathrm{C}), 112.4(\mathrm{C}), 118.8(\mathrm{C}), 131.9(\mathrm{CH}), 137.8(\mathrm{CH})$, 138.0 (C). GC-MS (EI) 269 ([M] $\left.]^{+}, 5 \%\right), 135$ (100\%), 107 (8\%), 93 (15\%), 79 (15\%), 67 (5\%), 55 (3\%). FT-IR (cm ${ }^{-1}$, neat, ATR) $3063(\mathrm{vw}), 2903(\mathrm{~s}), 2847(\mathrm{~m}), 2227(\mathrm{w}), 1299(\mathrm{w}), 1038(\mathrm{~m})$, $851(\mathrm{~m}), 831$ (s), 556 (vs). HRMS (CI+) calcd for $\mathrm{C}_{17} \mathrm{H}_{20} \mathrm{NS}[\mathrm{M}+\mathrm{H}]^{+}$: 270.1311, found: 270.1307.

\footnotetext{
${ }^{36}$ Ahbala, M.; Hapiot, P.; Houmam, A.; Jouini, M.; Pinson, J.; Saveant, J.-M. J. Am. Chem. Soc., 1995, 117, 11488.
} 


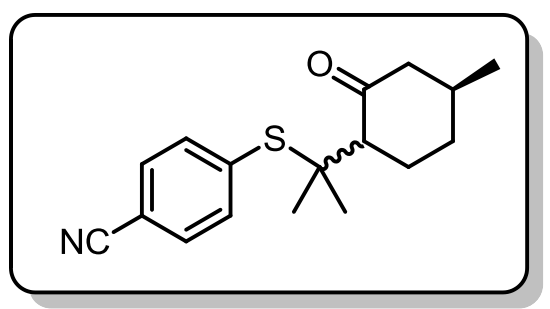

trans and cis 4-((2-(4-Methyl-2-oxocyclohexyl)propan-2yl)thio)benzonitrile, 3k \& 3k' (124 mg, 86\% combined yield of both diastereomers) was prepared according to the general procedure for thioetherification from 4-bromobenzonitrile (91 $\mathrm{mg}, \quad 0.50 \mathrm{mmol})$ and a diastereomeric mixture of 8mercaptomenthone $(112 \mathrm{mg}, 0.6 \mathrm{mmol})$. The diasteromeric thioethers were separated by flash column chromatography to give trans (86 mg, 60\%) and cis (38 mg, 26\%) as clear, pale yellow oils. Ratio: 30:70 cis:trans.

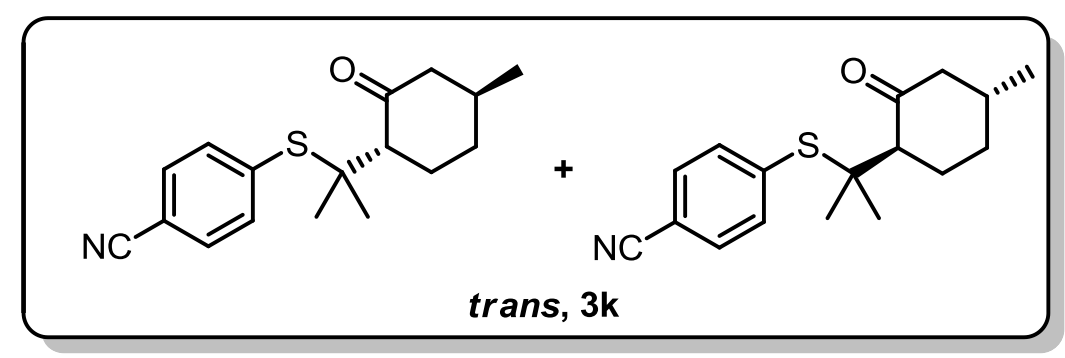

(1S,4S)- \& (1R,4R)-4-((2-(4-Methyl-2-oxocyclohexyl)propan-2-yl)thio)benzonitrile

${ }^{1} \mathrm{H}$ NMR $\left(\mathrm{CDCl}_{3}, 500 \mathrm{MHz}\right) \delta 1.02(\mathrm{~d}, J=6.2 \mathrm{~Hz}, 3 \mathrm{H}), 1.39(\mathrm{~s}, 3 \mathrm{H}), 1.43(\mathrm{~s}, 3 \mathrm{H}), 1.62(\mathrm{qd}, J=$ 13.2, $3.3 \mathrm{~Hz}, 1 \mathrm{H}), 1.86-2.03$ (m, 3H), 2.29 (ddd, $J=11.7,3.7,2.0 \mathrm{~Hz}, 1 \mathrm{H}), 2.35$ (dd, $J=13.2$, $4.4 \mathrm{~Hz}, 1 \mathrm{H}), 2.67(\mathrm{dq}, J=13.4,3.6 \mathrm{~Hz}, 1 \mathrm{H}), 7.59(\mathrm{~d}, J=8.6 \mathrm{~Hz}, 2 \mathrm{H}), 7.62(\mathrm{~d}, J=8.3 \mathrm{~Hz}, 2 \mathrm{H})$. ${ }^{13} \mathrm{C} \mathrm{NMR}\left(\mathrm{CDCl}_{3}, 125 \mathrm{MHz}\right) \delta 22.4\left(\mathrm{CH}_{3}\right), 24.8\left(\mathrm{CH}_{2}\right), 28.4\left(\mathrm{CH}_{3}\right), 30.0\left(\mathrm{CH}_{3}\right), 34.8(\mathrm{CH}), 37.0$ $\left(\mathrm{CH}_{2}\right), 52.5\left(\mathrm{CH}_{2}\right), 52.9(\mathrm{C}), 58.1(\mathrm{CH}), 112.8(\mathrm{C}), 118.6(\mathrm{C}), 132.2(\mathrm{CH}), 137.8(\mathrm{CH}), 139.2$ (C), 210.3 (C). GC-MS (EI) 287 ([M] $\left.{ }^{+}, 9 \%\right), 153$ (100\%), 135 (47\%), 109 (74\%), 95 (12\%), 93 (11\%), 91 (11\%), $81(39 \%), 69$ (78\%), 67 (21\%), 55 (16\%). FT-IR (cm ${ }^{-1}$, neat, ATR) $2954(\mathrm{~m})$, 2871 (w), 2229 (m), 1709 (vs), 1455 (m), 1119 (m), 1085 (m), 834 (s), 546 (s). HRMS (ES+) calcd for $\mathrm{C}_{17} \mathrm{H}_{21} \mathrm{NOSNa}[\mathrm{M}+\mathrm{Na}]^{+}: 310.1242$, found: 310.1243 .

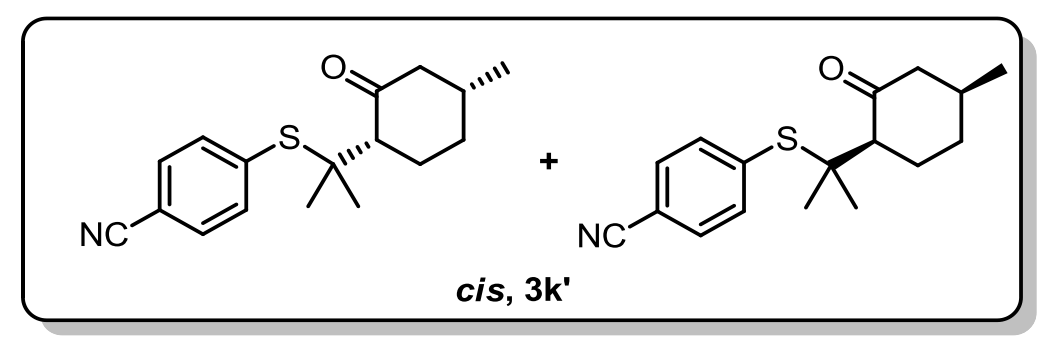

(1R,4S)- \& (1R,4S)-4-((2-(4-Methyl-2-oxocyclohexyl)propan-2-yl)thio)benzonitrile

${ }^{1} \mathrm{H} \mathrm{NMR}\left(\mathrm{CDCl}_{3}, 500 \mathrm{MHz}\right) \delta 0.98(\mathrm{~d}, J=7.1 \mathrm{~Hz}, 3 \mathrm{H}), 1.42(\mathrm{~s}, 3 \mathrm{H}), 1.43(\mathrm{~s}, 3 \mathrm{H}), 1.67$ - $1.75(\mathrm{~m}$, $1 \mathrm{H}), 1.85-2.00(\mathrm{~m}, 2 \mathrm{H}), 2.10(\mathrm{ddd}, J=12.7,3.9,1.7 \mathrm{~Hz}, 1 \mathrm{H}), 2.40(\mathrm{dd}, J=11.1,5.0 \mathrm{~Hz}, 2 \mathrm{H}), 2.44$ - 2.53 (m, 2H), 7.62 (br s, 4H). ${ }^{13} \mathrm{C} \mathrm{NMR}\left(\mathrm{CDCl}_{3}, 125 \mathrm{MHz}\right) \delta$. GC-MS (EI) $287\left([\mathrm{M}]^{+}, 10 \%\right)$, 153 (100\%), 135 (45\%), 109 (71\%), 95 (13\%), 93 (10\%), 91 (12\%), 81 (41\%), 69 (75\%), 67 (19\%), 55 (12\%). FT-IR ( $\mathrm{cm}^{-1}$, neat, ATR) $2957(\mathrm{~m}), 2873(\mathrm{w}), 2229(\mathrm{~m}), 1709$ (vs), $1456(\mathrm{~m})$, 1119 (m), 1085 (m), 833 (s), 554 (s). HRMS (ES+) calcd for $\mathrm{C}_{17} \mathrm{H}_{21} \mathrm{NOSNa}[\mathrm{M}+\mathrm{Na}]^{+}: 310.1242$, found: 310.1245 . 


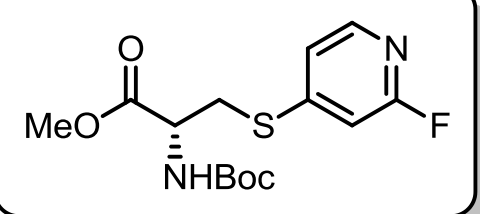

desired thioether was obtained as a white powder $\left(\mathrm{mp}=58^{\circ} \mathrm{C}\right) \cdot[\alpha]^{23} \mathrm{D}=+17.22\left(\mathrm{CHCl}_{3}, c=\right.$ $1.00)$.

${ }^{1} \mathrm{H}$ NMR $\left(\mathrm{CDCl}_{3}, 500 \mathrm{MHz}\right) \delta 1.42(\mathrm{~s}, 9 \mathrm{H}), 3.41(\mathrm{dd}, J=13.7,3.9 \mathrm{~Hz}, 1 \mathrm{H}), 3.57(\mathrm{dd}, J=13.9$, $4.4 \mathrm{~Hz}, 1 \mathrm{H}), 3.74(\mathrm{~s}, 3 \mathrm{H}), 4.61-4.70(\mathrm{~m}, 1 \mathrm{H}), 5.36-5.44(\mathrm{~m}, 1 \mathrm{H}), 6.81(\mathrm{~s}, 1 \mathrm{H}), 7.04(\mathrm{~d}, J=5.4$ $\mathrm{Hz}, 1 \mathrm{H}), 8.01(\mathrm{~d}, J=5.4 \mathrm{~Hz}, 1 \mathrm{H}) .{ }^{13} \mathrm{C} \mathbf{N M R}\left(\mathrm{CDCl}_{3}, 125 \mathrm{MHz}\right) \delta 28.4\left(\mathrm{CH}_{3}\right), 33.8\left(\mathrm{CH}_{2}\right), 53.1$ $\left(\mathrm{CH}_{3}\right), 53.4(\mathrm{CH}), 80.9(\mathrm{C}), 106.4\left(\mathrm{~d}, J_{\mathrm{C}-\mathrm{C}-\mathrm{F}}=39.4 \mathrm{~Hz}, \mathrm{CH}\right), 119.3\left(\mathrm{~d}, J_{\mathrm{C}-\mathrm{C}-\mathrm{C}-\mathrm{C}-\mathrm{F}}=3.7 \mathrm{~Hz}, \mathrm{CH}\right)$, $147.3\left(\mathrm{~d}, J_{\mathrm{C}-\mathrm{N}-\mathrm{C}-\mathrm{F}}=16.5 \mathrm{~Hz}, \mathrm{CH}\right), 153.4(\mathrm{C}), 155.2(\mathrm{C}), 164.1\left(\mathrm{~d}, J_{\mathrm{C}-\mathrm{F}}=239.2 \mathrm{~Hz}, \mathrm{CF}\right), 170.6$ (C). ${ }^{19}$ F NMR $\left(\mathrm{CDCl}_{3}, 282 \mathrm{MHz}\right) \delta$-71.08 (s, 1F). FT-IR $\left(\mathrm{cm}^{-1}\right.$, neat, ATR) 3355 (w, br), 2964 (w), 1745 (m), 1705 (s, br), 1590 (s), 1251 (s), 1217 (s), 1159 (vs), 900 (m). HRMS (ES+) calcd for $\mathrm{C}_{14} \mathrm{H}_{20} \mathrm{FN}_{2} \mathrm{O}_{4} \mathrm{~S}[\mathrm{M}+\mathrm{H}]^{+}:$331.1128, found: 331.1129). $[\alpha]^{23}{ }_{\mathrm{D}}=+17.22\left(\mathrm{CHCl}_{3}, c=1.00\right)$.

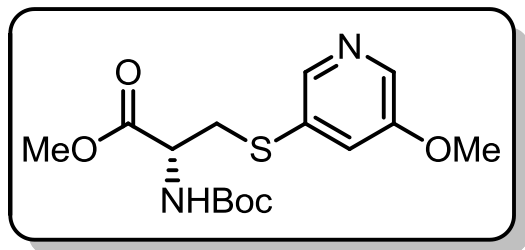

\section{(R)-Methyl}

2-((tert-Butoxycarbonyl)amino)-3-((5methoxypyridin-3-yl)thio)propanoate, 3m (127 mg, 90\%) was prepared according to the general procedure for thioetherification from 3-bromo-5-methoxypyridine $(77 \mathrm{mg}$, $0.41 \mathrm{mmol})$ and $(R)$-methyl 2-((tert-butoxycarbonyl)amino)-3mercaptopropanoate $(115 \mathrm{mg}, 0.49 \mathrm{mmol})$. The desired thioether was obtained as a clear, colorless oil. $[\alpha]^{23}=+46.7\left(\mathrm{CHCl}_{3}, c=1.00\right)$.

${ }^{1} \mathrm{H} \mathrm{NMR}\left(\mathrm{CDCl}_{3}, 500 \mathrm{MHz}\right) \delta 1.42(\mathrm{~s}, 9 \mathrm{H}), 3.35(\mathrm{dd}, J=13.7,4.7 \mathrm{~Hz}, 1 \mathrm{H}), 3.42(\mathrm{dd}, J=13.7$, $4.7 \mathrm{~Hz}, 1 \mathrm{H}), 3.63$ (s, 3H), $3.86(\mathrm{~s}, 3 \mathrm{H}), 4.52$ - $4.62(\mathrm{~m}, 1 \mathrm{H}), 5.30$ - $5.40(\mathrm{~m}, 1 \mathrm{H}), 7.27(\mathrm{~s}, 1 \mathrm{H})$, $8.17(\mathrm{~d}, J=2.7 \mathrm{~Hz}, 1 \mathrm{H}), 8.23(\mathrm{~d}, J=1.7 \mathrm{~Hz}, 1 \mathrm{H}) .{ }^{13} \mathrm{C} \mathrm{NMR}\left(\mathrm{CDCl}_{3}, 125 \mathrm{MHz}\right) \delta 28.5\left(\mathrm{CH}_{3}\right)$, $37.4\left(\mathrm{CH}_{2}\right), 52.8\left(\mathrm{CH}_{3}\right), 53.6(\mathrm{CH}), 55.9\left(\mathrm{CH}_{3}\right), 80.6(\mathrm{C}), 122.9(\mathrm{CH}), 132.6(\mathrm{C}), 136.6(\mathrm{CH})$, $143.8(\mathrm{CH}), 155.2(\mathrm{C}), 155.8(\mathrm{C}), 171.1(\mathrm{C})$. FT-IR $\left(\mathrm{cm}^{-1}\right.$, neat, ATR) 3365 (vw, br), 2967 (w), 1746 (m), 1705 (s), 1505 (m), 1265 (s), 1220 (s), 1160 (vs), 1013 (m), 859 (w), 701 (w). HRMS (ES+) calcd for $\mathrm{C}_{15} \mathrm{H}_{23} \mathrm{~N}_{2} \mathrm{O}_{5} \mathrm{~S}[\mathrm{M}+\mathrm{H}]^{+}: 343.1328$, found: 343.1331 .

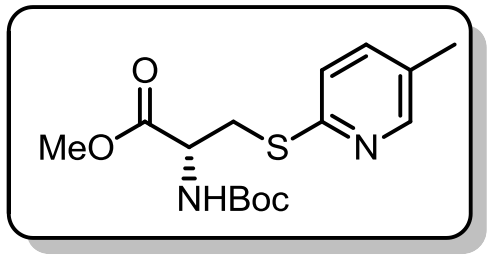

(R)-Methyl

2-((tert-Butoxycarbonyl)amino)-3-((5methylpyridin-2-yl)thio)propanoate, 3n (158 mg, 97\%) was prepared according to the general procedure for thioetherification from 2-bromo-5-methylpyridine $(86 \mathrm{mg}, 0.50 \mathrm{mmol})$ and $(R)$ methyl 2-((tert-butoxycarbonyl)amino)-3-mercaptopropanoate (141 $\mathrm{mg}, 0.60 \mathrm{mmol})$. The desired thioether was obtained as a white solid $\left(\mathrm{mp}=60{ }^{\circ} \mathrm{C}\right)$. $[\alpha]^{23} \mathrm{D}=+14.0\left(\mathrm{CHCl}_{3}, c=1.00\right)$.

${ }^{1} \mathrm{H}$ NMR $\left(\mathrm{CDCl}_{3}, 500 \mathrm{MHz}\right) \delta 1.37(\mathrm{~s}, 9 \mathrm{H}), 2.22(\mathrm{~s}, 3 \mathrm{H}), 3.39-3.60(\mathrm{~m}, 2 \mathrm{H}), 3.66(\mathrm{~s}, 3 \mathrm{H}), 4.30$ - $4.59(\mathrm{~m}, 1 \mathrm{H}), 6.29-6.52(\mathrm{~m}, 1 \mathrm{H}), 7.07(\mathrm{~d}, J=8.1 \mathrm{~Hz}, 1 \mathrm{H}), 7.27(\mathrm{dd}, J=8.0,2.3 \mathrm{~Hz}, 1 \mathrm{H}), 8.20$ (s, $1 \mathrm{H}) .{ }^{13} \mathrm{C}$ NMR $\left(\mathrm{CDCl}_{3}, 125 \mathrm{MHz}\right) \delta 17.9\left(\mathrm{CH}_{3}\right), 28.4\left(\mathrm{CH}_{3}\right), 32.5\left(\mathrm{CH}_{2}\right), 52.4\left(\mathrm{CH}_{3}\right), 54.6$ $(\mathrm{CH}), 79.7(\mathrm{C}), 122.4(\mathrm{CH}), 129.8(\mathrm{C}), 137.4(\mathrm{CH}), 149.6(\mathrm{CH}), 154.3(\mathrm{C}), 155.6(\mathrm{C}), 171.7(\mathrm{C})$. FT-IR $\left(\mathrm{cm}^{-1}\right.$, neat, ATR) 3351 (w, br), $2978(\mathrm{w}), 1745$ (m), 1712 (s), 1505 (m), 1464 (s), 1365 (s), 1162 (vs), $1111(\mathrm{~m}) 820(\mathrm{w}), 490(\mathrm{w})$. HRMS (ES+) calcd for $\mathrm{C}_{15} \mathrm{H}_{23} \mathrm{~N}_{2} \mathrm{O}_{4} \mathrm{~S}[\mathrm{M}+\mathrm{H}]^{+}$: 327.1379, found: 327.1367 . 


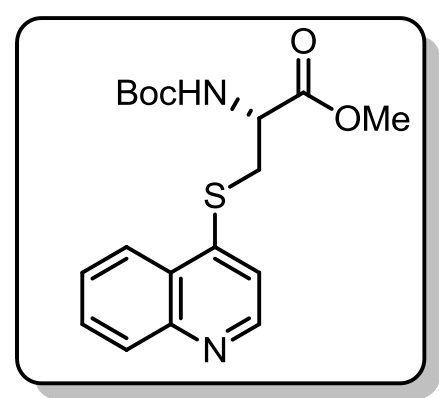

(R)-Methyl 2-((tert-Butoxycarbonyl)amino)-3-(quinolin-4ylthio)propanoate, 30 (133 $\mathrm{mg}, 73 \%)$ was prepared according to the general procedure for thioetherification from 4-bromoquinoline $(104 \mathrm{mg}, \quad 0.50 \quad \mathrm{mmol})$ and $(R)$-methyl 2-((tertbutoxycarbonyl)amino)-3-mercaptopropanoate $\quad(141 \mathrm{mg}, \quad 0.60$ $\mathrm{mmol})$. The desired thioether was obtained as a clear, yellow oil. $[\alpha]_{\mathrm{D}}^{23}=+36.6\left(\mathrm{CHCl}_{3}, c=1.00\right)$.

${ }^{1} \mathrm{H}$ NMR $\left(\mathrm{CDCl}_{3}, 500 \mathrm{MHz}\right) \delta 1.41(\mathrm{~s}, 9 \mathrm{H}), 3.54(\mathrm{dd}, J=13.7,4.7$ $\mathrm{Hz}, 1 \mathrm{H}), 3.67(\mathrm{dd}, J=13.7,4.9 \mathrm{~Hz}, 1 \mathrm{H}), 3.70(\mathrm{~s}, 3 \mathrm{H}), 4.74(\mathrm{q}, J=$ $5.9 \mathrm{~Hz}, 1 \mathrm{H}), 5.43(\mathrm{~d}, J=6.9 \mathrm{~Hz}, 1 \mathrm{H}), 7.34(\mathrm{~d}, J=4.4 \mathrm{~Hz}, 1 \mathrm{H}), 7.56(\mathrm{t}, J=7.7 \mathrm{~Hz}, 1 \mathrm{H}), 7.72(\mathrm{t}$, $J=7.6 \mathrm{~Hz}, 1 \mathrm{H}), 8.08(\mathrm{~d}, J=8.6 \mathrm{~Hz}, 1 \mathrm{H}), 8.15(\mathrm{~d}, J=8.3 \mathrm{~Hz}, 1 \mathrm{H}), 8.74(\mathrm{~d}, J=4.7 \mathrm{~Hz}, 1 \mathrm{H}) .{ }^{13} \mathrm{C}$ NMR $\left(\mathrm{CDCl}_{3}, 125 \mathrm{MHz}\right) \delta 28.5\left(\mathrm{CH}_{3}\right), 34.4\left(\mathrm{CH}_{2}\right), 53.0\left(\mathrm{CH}_{3}\right), 53.3(\mathrm{CH}), 80.7(\mathrm{C}), 117.8$ $(\mathrm{CH}), 124.1(\mathrm{CH}), 126.8(\mathrm{~s}, 3 \mathrm{C}), 127.1(\mathrm{C}), 130.1(\mathrm{CH}), 130.3(\mathrm{CH}), 146.1(\mathrm{C}), 147.9(\mathrm{C}), 149.6$ (CH), $155.2(\mathrm{C}), 170.9(\mathrm{C})$. FT-IR ( $\mathrm{cm}^{-1}$, neat, ATR) 3355 (w, br), $2964(\mathrm{w}), 1745(\mathrm{~m}), 1705(\mathrm{~s})$, 1563 (m), 1497 (s), 1366 (m), 1247 (m), 1160 (vs), 1051 (m), 823 (m), 757 (s), 730 (s), 667 (m). HRMS (ES+) calcd for $\mathrm{C}_{18} \mathrm{H}_{23} \mathrm{~N}_{2} \mathrm{O}_{4} \mathrm{~S}[\mathrm{M}+\mathrm{H}]^{+}: 363.1379$, found: 363.1385 .

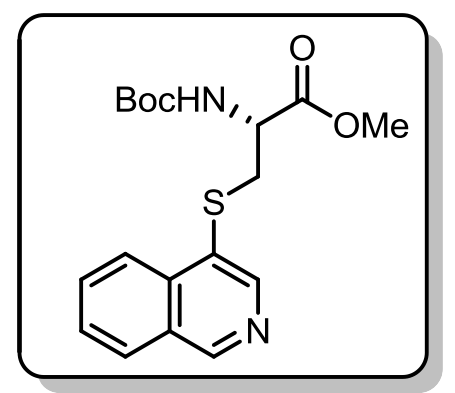

(R)-Methyl 2-((tert-Butoxycarbonyl)amino)-3-(isoquinolin-4ylthio)propanoate, $3 \mathbf{p}(111 \mathrm{mg}, 83 \%)$ was prepared according to the general procedure for thioetherification from 4bromoisoquinoline $(78 \mathrm{mg}, 0.37 \mathrm{mmol})$ and $(R)$-methyl $2-(($ tertbutoxycarbonyl)amino)-3-mercaptopropanoate $\quad(106 \mathrm{mg}, \quad 0.45$ mmol). The desired thioether was obtained as an off white powder $\left(\mathrm{mp}=89^{\circ} \mathrm{C}\right) .[\alpha]^{23} \mathrm{D}=+76.5\left(\mathrm{CHCl}_{3}, c=1.00\right)$.

${ }^{1} \mathrm{H} \mathrm{NMR}\left(\mathrm{CDCl}_{3}, 500 \mathrm{MHz}\right) \delta 1.36(\mathrm{~s}, 9 \mathrm{H}), 3.37$ (dd, $J=13.4,4.6$ $\mathrm{Hz}, 1 \mathrm{H}), 3.46$ (dd, $J=14.0,4.0 \mathrm{~Hz}, 1 \mathrm{H}), 3.52(\mathrm{~s}, 3 \mathrm{H}), 4.55$ (br q, $J$ $=7.0 \mathrm{~Hz}, 1 \mathrm{H}), 5.39(\mathrm{br} \mathrm{d}, J=6.7 \mathrm{~Hz}, 1 \mathrm{H}), 7.64(\mathrm{t}, J=7.5 \mathrm{~Hz}, 1 \mathrm{H}), 7.79(\mathrm{t}, J=7.3 \mathrm{~Hz}, 1 \mathrm{H})$, $7.97(\mathrm{~d}, J=7.9 \mathrm{~Hz}, 1 \mathrm{H}), 8.33(\mathrm{~d}, J=8.5 \mathrm{~Hz}, 1 \mathrm{H}), 8.66(\mathrm{~s}, 1 \mathrm{H}), 9.15(\mathrm{~s}, 1 \mathrm{H}) .{ }^{13} \mathrm{C}$ NMR $\left(\mathrm{CDCl}_{3}\right.$, $125 \mathrm{MHz}) \delta 28.5\left(\mathrm{CH}_{3}\right), 38.0\left(\mathrm{CH}_{2}\right), 52.7\left(\mathrm{CH}_{3}\right), 53.8(\mathrm{CH}), 80.4(\mathrm{C}), 124.7(\mathrm{CH}), 127.2(\mathrm{C})$, $128.1(\mathrm{CH}), 128.5(\mathrm{CH}), 129.0(\mathrm{C}), 131.5(\mathrm{CH}), 136.6(\mathrm{C}), 147.5(\mathrm{CH}), 153.0(\mathrm{CH}), 155.1(\mathrm{C})$, 171.1 (C). FT-IR ( $\mathrm{cm}^{-1}$, neat, ATR) $3353(\mathrm{w}), 2962(\mathrm{vw}), 1738(\mathrm{~s}), 1713(\mathrm{~s}), 1521(\mathrm{~s}), 1225(\mathrm{~m})$, 1158 (vs), 1014 (m), 782 (m), 753 (m). HRMS (ES+) calcd for $\mathrm{C}_{18} \mathrm{H}_{23} \mathrm{~N}_{2} \mathrm{O}_{4} \mathrm{~S}[\mathrm{M}+\mathrm{H}]^{+}$: 363.1379, found: 363.1386 .

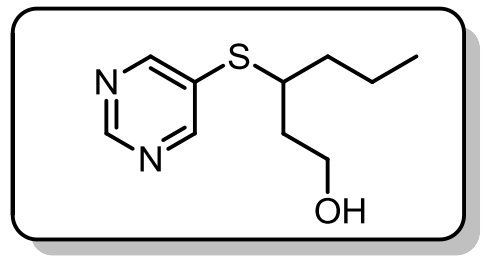

3-(Pyrimidin-5-ylthio)hexan-1-ol, 3q (74 $\mathrm{mg}, \quad 70 \%)$ was prepared according to the general procedure for thioetherification from 5-bromopyrimidine $(80 \mathrm{mg}, \quad 0.50 \mathrm{mmol})$ and 3 mercaptohexan-1-ol $(83.0 \mu \mathrm{L}, 80 \mathrm{mg}, 0.60 \mathrm{mmol})$. The desired thioether was obtained as a clear, yellow oil.

${ }^{1} \mathrm{H}$ NMR $\left(\mathrm{CDCl}_{3}, 500 \mathrm{MHz}\right) \delta 0.90(\mathrm{t}, J=7.30 \mathrm{~Hz}, 3 \mathrm{H}), 1.42-$ $1.66(\mathrm{~m}, 4 \mathrm{H}), 1.75$ (ddt, $J=14.3,8.5,5.6 \mathrm{~Hz}, 1 \mathrm{H}), 1.90$ (ddt, $J=14.6,7.5,5.5 \mathrm{~Hz}, 1 \mathrm{H}), 2.20(\mathrm{br}$ $\mathrm{s}, 1 \mathrm{H}), 3.33$ (ddt, $J=8.2,7.2,5.5 \mathrm{~Hz}, 1 \mathrm{H}), 3.76-3.83(\mathrm{~m}, 1 \mathrm{H}), 3.84-3.91(\mathrm{~m}, 1 \mathrm{H}), 8.73(\mathrm{~s}, 2 \mathrm{H})$, $9.03(\mathrm{~s}, 1 \mathrm{H}) .{ }^{13} \mathrm{C}$ NMR $\left(\mathrm{CDCl}_{3}, 125 \mathrm{MHz}\right) \delta 14.1\left(\mathrm{CH}_{3}\right), 20.2\left(\mathrm{CH}_{2}\right), 37.5\left(\mathrm{CH}_{2}\right), 37.6\left(\mathrm{CH}_{2}\right)$, $46.3(\mathrm{CH}), 60.0\left(\mathrm{CH}_{2}\right), 132.2(\mathrm{C}), 156.6(\mathrm{CH}), 159.4(\mathrm{CH}) . \mathrm{GC}-\mathrm{MS}(\mathrm{EI}) 212\left([\mathrm{M}]^{+}, 72 \%\right), 148$ (7\%), $125(10 \%), 112(100 \%), 83(58 \%), 68(9 \%), 57(24 \%), 55(99 \%)$. FT-IR $\left(\mathrm{cm}^{-1}\right.$, neat, 
ATR) 3368 (m, br), 3040 (vw), 2931 (m), 2872 (m), 1541 (s), 1398 (s), 1162 (w), 1030 (s), 719 (vs), 626 (s). HRMS (ES+) calcd for $\mathrm{C}_{10} \mathrm{H}_{17} \mathrm{~N}_{2} \mathrm{OS}[\mathrm{M}+\mathrm{H}]^{+}$: 213.1062, found: 213.1068.

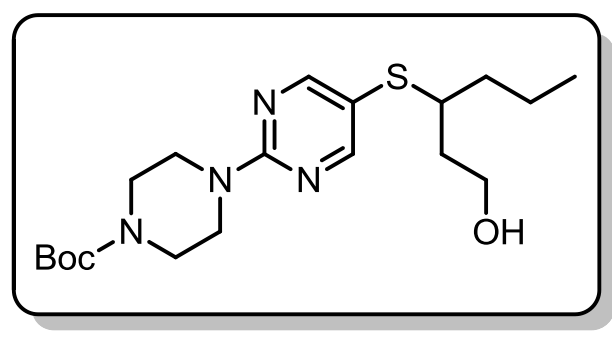

tert-Butyl 4-(5-((1-hydroxyhexan-3-yl)thio)pyrimidin2-yl)piperazine-1-carboxylate, 3r (136 mg, 69\%) was prepared according to the general procedure for thioetherification from tert-butyl 4-(5-bromopyrimidin-2yl)piperazine-1-carboxylate $(172 \mathrm{mg}, 0.50 \mathrm{mmol})$ and $3-$ mercaptohexan-1-ol $(83.0 \mu \mathrm{L}, 80 \mathrm{mg}, 0.60 \mathrm{mmol})$. The desired thioether was obtained as a white solid $(\mathrm{mp}=56$ $\left.{ }^{\circ} \mathrm{C}\right)$.

${ }^{1} \mathrm{H}$ NMR $\left(\mathrm{CDCl}_{3}, 500 \mathrm{MHz}\right) \delta 0.91(\mathrm{t}, J=7.1 \mathrm{~Hz}, 3 \mathrm{H}), 1.42-1.60(\mathrm{~m}, 4 \mathrm{H}), 1.49(\mathrm{~s}, 9 \mathrm{H}), 1.60$ $1.71(\mathrm{~m}, 1 \mathrm{H}), 1.72-1.82(\mathrm{~m}, 2 \mathrm{H}), 2.79-2.89(\mathrm{~m}, 1 \mathrm{H}), 3.50(\mathrm{t}, J=4.6 \mathrm{~Hz}, 4 \mathrm{H}), 3.82(\mathrm{t}, J=4.6$ $\mathrm{Hz}, 5 \mathrm{H}), 3.87-3.96(\mathrm{~m}, 1 \mathrm{H}), 8.35(\mathrm{~s}, 2 \mathrm{H}) .{ }^{13} \mathrm{C} \mathrm{NMR}\left(\mathrm{CDCl}_{3}, 125 \mathrm{MHz}\right) \delta 14.1\left(\mathrm{CH}_{3}\right), 20.2$ $\left(\mathrm{CH}_{2}\right), 28.7\left(\mathrm{CH}_{3}\right), 37.1\left(\mathrm{CH}_{2} \times 2\right), 43.9\left(\mathrm{CH}_{2} \times 2\right), 47.5(\mathrm{CH}), 60.8\left(\mathrm{CH}_{2}\right), 80.4(\mathrm{C}), 114.3(\mathrm{C})$, 155.1 (C), 160.9 (C), 164.0 (CH). FT-IR (cm ${ }^{-1}$, neat, ATR) 3423 (w, br), 2942 (w), 2863 (w), 1697 (m), 1575 (vs), 1502 (s), 1418 (s), 1242 (vs), 1125 (s), 996 (m), 794 (w). HRMS (ES+) calcd for $\mathrm{C}_{19} \mathrm{H}_{32} \mathrm{~N}_{4} \mathrm{O}_{3} \mathrm{SNa}[\mathrm{M}+\mathrm{Na}]^{+}$: 419.2093, found: 419.2093 .

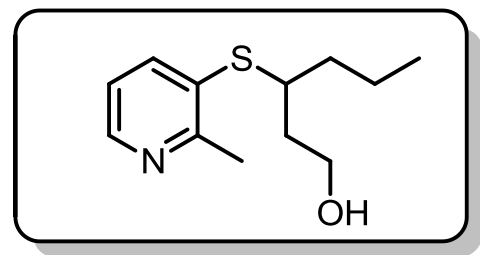

3-((3-Methylpyridin-2-yl)thio)hexan-1-ol, 3s (60 mg, 53\%) was prepared according to the general procedure for thioetherification from 3-bromo-2-methylpyridine (86 $\mathrm{mg}, 0.50 \mathrm{mmol})$ and 3 mercaptohexan-1-ol $(83.0 \mu \mathrm{L}, 80 \mathrm{mg}, 0.60 \mathrm{mmol})$. The desired thioether was obtained as a clear, colorless oil.

${ }^{1} \mathrm{H}$ NMR $\left(\mathrm{CDCl}_{3}, 500 \mathrm{MHz}\right) \delta 0.91(\mathrm{t}, J=7.3 \mathrm{~Hz}, 3 \mathrm{H}), 1.45-$ $1.53(\mathrm{~m}, 2 \mathrm{H}), 1.56-1.69(\mathrm{~m}, 2 \mathrm{H}), 1.82(\mathrm{ddt}, J=14.3,7.8,6.2 \mathrm{~Hz}, 1 \mathrm{H}), 1.93$ (ddt, $J=14.6,7.5$, $5.5 \mathrm{~Hz}, 1 \mathrm{H}), 2.11$ (br s, 1H), $2.62(\mathrm{~s}, 3 \mathrm{H}), 3.35(\mathrm{qd}, J=6.8,6.1 \mathrm{~Hz}, 1 \mathrm{H}), 3.75-3.89(\mathrm{~m}, 2 \mathrm{H})$, $7.07(\mathrm{dd}, J=7.8,4.7 \mathrm{~Hz}, 1 \mathrm{H}), 7.66(\mathrm{dd}, J=7.8,1.5 \mathrm{~Hz}, 1 \mathrm{H}), 8.29(\mathrm{dd}, J=4.9,1.5 \mathrm{~Hz}, 1 \mathrm{H}) .{ }^{13} \mathrm{C}$ NMR $\left(\mathrm{CDCl}_{3}, 125 \mathrm{MHz}\right) \delta 14.2\left(\mathrm{CH}_{3}\right), 20.2\left(\mathrm{CH}_{2}\right), 23.6\left(\mathrm{CH}_{3}\right), 37.4\left(\mathrm{CH}_{2}\right), 37.5\left(\mathrm{CH}_{2}\right), 44.9$ $(\mathrm{CH}), 60.4\left(\mathrm{CH}_{2}\right), 121.7(\mathrm{CH}), 132.3(\mathrm{C}), 137.8(\mathrm{CH}), 146.4(\mathrm{CH}), 158.6(\mathrm{C})$. GC-MS (EI) 225 ([M] $\left.]^{+}, 63 \%\right), 207(1 \%), 152(2 \%), 138(1 \%), 125(100 \%), 92(4 \%), 79(5 \%), 55$ (19\%). FT-IR $\left(\mathrm{cm}^{-1}\right.$, neat, ATR) 3270 (w, br), 2931 (m), 1566 (w), 1420 (vs), 1053 (vs), 791 (m), 725 (s). HRMS (ES+) calcd for $\mathrm{C}_{12} \mathrm{H}_{20} \mathrm{NOS}[\mathrm{M}+\mathrm{H}]^{+}: 226.1266$, found: 226.1257 .

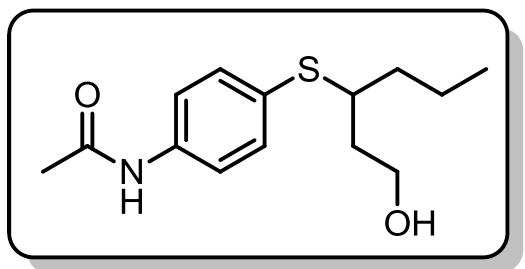

N-(4-((1-Hydroxyhexan-3-yl)thio)phenyl)acetamide, 3t (69 $\mathrm{mg}, 52 \%)$ was prepared according to the general procedure for thioetherification from $N$-(4-bromophenyl)acetamide (107 $\mathrm{mg}$, $0.50 \mathrm{mmol})$ and 3-mercaptohexan-1-ol $(83.0 \mu \mathrm{L}, 80 \mathrm{mg}, 0.60$ $\mathrm{mmol})$. The desired thioether was obtained as a white solid (mp $\left.=96^{\circ} \mathrm{C}\right)$.

${ }^{1} \mathrm{H}$ NMR $\left(\mathrm{CDCl}_{3}, 500 \mathrm{MHz}\right) \delta 0.89(\mathrm{t}, J=6.6 \mathrm{~Hz}, 3 \mathrm{H}), 1.42-1.58(\mathrm{~m}, 4 \mathrm{H}), 1.68-1.78(\mathrm{~m}, 1 \mathrm{H})$, 1.80 - $1.89(\mathrm{~m}, 1 \mathrm{H}), 2.14(\mathrm{~s}, 4 \mathrm{H}), 3.11-3.17(\mathrm{~m}, 1 \mathrm{H}), 3.74-3.82(\mathrm{~m}, 1 \mathrm{H}), 3.82-3.90(\mathrm{~m}, 1 \mathrm{H})$, $7.36(\mathrm{~d}, J=8.8 \mathrm{~Hz}, 2 \mathrm{H}), 7.43(\mathrm{~d}, J=8.3 \mathrm{~Hz}, 2 \mathrm{H}), 7.66-7.82(\mathrm{~m}, 1 \mathrm{H}) .{ }^{13} \mathrm{C} \mathrm{NMR}\left(\mathrm{CDCl}_{3}, 125\right.$ MHz) $\delta 14.2\left(\mathrm{CH}_{3}\right), 20.3\left(\mathrm{CH}_{2}\right), 24.7\left(\mathrm{CH}_{3}\right), 37.5\left(\mathrm{CH}_{2} \times 2\right), 47.0(\mathrm{CH}), 60.9\left(\mathrm{CH}_{2}\right), 120.6(\mathrm{CH})$, 129.9 (C), 134.0 (CH), 137.5 (C), 168.9 (C). FT-IR (cm ${ }^{-1}$, neat, ATR) 3299 (w), 2957 (w), 2931 
(w), 1667 (m), 1591 (s), 1529 (s), 1493 (s), 1395 (m) 1312 (s), 1038 (w), 825 (m), 730 (vs). HRMS (ES+) calcd for $\mathrm{C}_{14} \mathrm{H}_{22} \mathrm{NO}_{2} \mathrm{~S}[\mathrm{M}+\mathrm{H}]^{+}: 268.1371$, found: 268.1370.

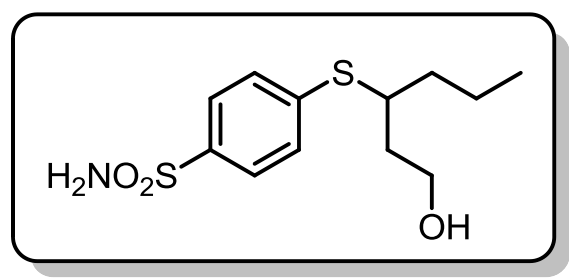

4-((1-Hydroxyhexan-3-yl)thio)benzenesulfonamide, 3u (139 $\mathrm{mg}, 96 \%$ ) was prepared according to the general procedure for thioetherification from 4bromobenzenesulfonamide (118 $\mathrm{mg}, 0.50 \mathrm{mmol})$ and 3 mercaptohexan-1-ol $(83.0 \mu \mathrm{L}, 80 \mathrm{mg}, 0.60 \mathrm{mmol})$. The desired thioether was obtained as a colorless oil.

${ }^{1} \mathrm{H}$ NMR $\left(\mathrm{CDCl}_{3}, 500 \mathrm{MHz}\right) \delta 0.90(\mathrm{t}, J=7.34 \mathrm{~Hz}, 3 \mathrm{H})$, $1.39-1.54(\mathrm{~m}, 2 \mathrm{H}), 1.54-1.69(\mathrm{~m}, 2 \mathrm{H}), 1.73-1.82(\mathrm{~m}, 1 \mathrm{H}), 1.85-1.94(\mathrm{~m}, 1 \mathrm{H}), 2.40$ (br s, $1 \mathrm{H}), 3.42-3.50(\mathrm{~m}, 1 \mathrm{H}), 3.65-3.83(\mathrm{~m}, 2 \mathrm{H}), 5.49$ (br s, $2 \mathrm{H}), 7.39(\mathrm{~d}, J=8.3 \mathrm{~Hz}, 2 \mathrm{H}), 7.73(\mathrm{~d}, J$ $=8.3 \mathrm{~Hz}, 2 \mathrm{H}),{ }^{13} \mathrm{C} \mathrm{NMR}\left(\mathrm{CDCl}_{3}, 125 \mathrm{MHz}\right) \delta 14.2\left(\mathrm{CH}_{3}\right), 20.3\left(\mathrm{CH}_{2}\right), 37.5\left(\mathrm{CH}_{2}\right), 37.6\left(\mathrm{CH}_{2}\right)$, $44.7(\mathrm{CH}), 60.4\left(\mathrm{CH}_{2}\right), 127.1(\mathrm{CH}), 129.4(\mathrm{CH}), 139.0(\mathrm{C}), 144.1(\mathrm{C})$. FT-IR $\left(\mathrm{cm}^{-1}\right.$, neat, ATR) 3262 (w, br), 2957 (w), 2932 (w), 1579 (w), 1323 (s), 1157 (vs), 1077 (s), 905 (w), 752 (m), 619 (s), 541 (s). HRMS (ES+) calcd for $\mathrm{C}_{12} \mathrm{H}_{20} \mathrm{NO}_{3} \mathrm{~S}_{2}[\mathrm{M}+\mathrm{H}]^{+}: 290.0885$, found: 290.0890 .

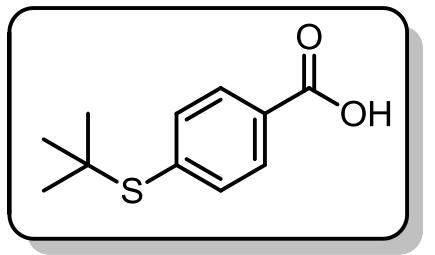

4-(tert-butylthio)benzoic acid, 3v (99 mg, 94\%) was prepared according to the general procedure for thioetherification from 4bromobenzoic acid (101 $\mathrm{mg}, 0.5 \mathrm{mmol})$ and tert-butylthiol $(67.6 \mu \mathrm{L}$, $54 \mathrm{mg}, 0.60 \mathrm{mmol})$ with the following modified workup: The reaction mixture was quenched with saturated aqueous $\mathrm{NH}_{4} \mathrm{Cl}$. The quenched reaction mixture was extracted with EtOAc $(3 \times 20 \mathrm{~mL})$. The solvent was removed in vacuo by rotary evaporation, giving a brown oil. The brown oil was taken up in $\sim 40 \mathrm{~mL}$ of $\mathrm{Et}_{2} \mathrm{O}$ and extracted with saturated aqueous $\mathrm{NaHCO}_{3}(3 \times 20 \mathrm{~mL})$. The combined aqueous layers were mixed with $\sim 40 \mathrm{~mL}$ of EtOAc and acidified by dropwise addition of concentrated $\mathrm{HCl}$ to $\mathrm{pH} 1(\sim 10 \mathrm{~mL})$. The layers were separated, and the aqueous layer was extracted with EtOAc $(3 \times 20 \mathrm{~mL})$. The combined organic layers were washed with deionized $\mathrm{H}_{2} \mathrm{O}(\sim 60 \mathrm{~mL})$ and dried $\left(\mathrm{MgSO}_{4}\right)$. The solvent was removed in vacuo by rotary evaporation. The resulting off-white solid was further purified by $\mathrm{SiO}_{2}$ column chromatography (gradient $\mathrm{CH}_{2} \mathrm{Cl}_{2}: \mathrm{MeOH}, 97: 3$ to $\left.95: 5\right)$, giving the desired thioether as a fluffy white powder (mp = 144 $\left.{ }^{\circ} \mathrm{C}\right)$.

${ }^{1} \mathrm{H}$ NMR $\left(\mathrm{CDCl}_{3}, 500 \mathrm{MHz}\right) \delta 1.34(\mathrm{~s}, 9 \mathrm{H}), 7.63(\mathrm{~d}, J=7.9 \mathrm{~Hz}, 2 \mathrm{H}), 8.06(\mathrm{~d}, J=7.9 \mathrm{~Hz}, 2 \mathrm{H})$, 12.65 (br. s, $1 \mathrm{H}) .{ }^{13} \mathrm{C}$ NMR $\left(\mathrm{CDCl}_{3}, 125 \mathrm{MHz}\right) \delta 31.4\left(\mathrm{CH}_{3}\right), 47.3(\mathrm{C}), 129.3(\mathrm{C}), 130.3(\mathrm{CH})$, $137.0(\mathrm{CH}), 140.7(\mathrm{CH}), 172.4(\mathrm{C})$. FT-IR $\left(\mathrm{cm}^{-1}\right.$, neat, ATR) 3120-2751 (m, very br), $2552(\mathrm{w})$, 1682 (vs) 1422 (s) 1277 (s), 1176 (w), 943 (m), 851 (w), 767 (m), 537 (w). HRMS (ES-) calcd for $\mathrm{C}_{11} \mathrm{H}_{13} \mathrm{O}_{2} \mathrm{~S}[\mathrm{M}-\mathrm{H}]^{-}: 209.0636$, found: 209.0639 .

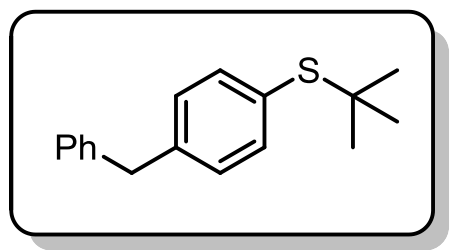

(4-Benzylphenyl)(tert-butyl)sulfane, 3w (102 mg, 80\%) was prepared according to the general procedure for thioetherification from 1-benzyl-4-bromobenzene $(124 \mathrm{mg}, 0.50 \mathrm{mmol})$ and tertbutylthiol $(67.6 \mu \mathrm{L}, 54 \mathrm{mg}, 0.60 \mathrm{mmol})$. The desired thioether was obtained as a clear, colorless oil.

${ }^{1} \mathrm{H}$ NMR $\left(\mathrm{CDCl}_{3}, 500 \mathrm{MHz}\right) \delta 1.28(\mathrm{~s}, 9 \mathrm{H}), 3.99(\mathrm{~s}, 2 \mathrm{H}), 7.15(\mathrm{~d}, J$ $=8.2 \mathrm{~Hz}, 2 \mathrm{H}), 7.17-7.24(\mathrm{~m}, 3 \mathrm{H}), 7.30(\mathrm{t}, J=7.6 \mathrm{~Hz}, 2 \mathrm{H}), 7.45(\mathrm{~d}, J=8.2 \mathrm{~Hz}, 2 \mathrm{H}) .{ }^{13} \mathrm{C} \mathrm{NMR}$ $\left(\mathrm{CDCl}_{3}, 125 \mathrm{MHz}\right) \delta 31.2\left(\mathrm{CH}_{3}\right), 41.9\left(\mathrm{CH}_{2}\right), 46.0(\mathrm{C}), 126.5(\mathrm{CH}), 128.8(\mathrm{CH}), 129.2(\mathrm{CH})$, 
$129.3(\mathrm{CH}), 130.4(\mathrm{C}), 137.8(\mathrm{CH}), 140.9(\mathrm{C}), 142.1(\mathrm{C})$. GC-MS (EI) $256\left([\mathrm{M}]^{+}, 31 \%\right), 200$ (100\%), 167 (87\%), $165(44 \%), 152(14 \%), 115(6 \%), 91(16 \%), 57(22 \%)$. FT-IR $\left(\mathrm{cm}^{-1}\right.$, neat, ATR) $3031(\mathrm{vw}), 2960(\mathrm{w}), 1490(\mathrm{w}), 1454(\mathrm{w}), 1167$ (w), 794 (w), $746(\mathrm{~m}), 724(\mathrm{~m}), 697(\mathrm{vs})$. HRMS (CI+) calcd for $\mathrm{C}_{17} \mathrm{H}_{20} \mathrm{~S}[\mathrm{M}]^{+}: 256.1286$, found: 256.1277 .

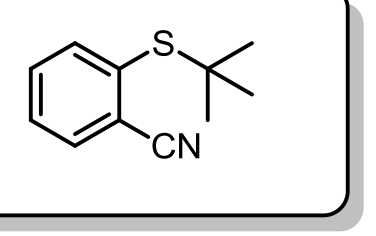

2-(tert-Butylthio)benzonitrile, 3x (71 $\mathrm{mg}, 74 \%)$ was prepared according to the general procedure for thioetherification from aryl halide $(91 \mathrm{mg}, 0.5 \mathrm{mmol})$ and tert-butylthiol $(67.6 \mu \mathrm{L}, 54 \mathrm{mg}, 0.60$ $\mathrm{mmol}$ ). The desired thioether was obtained as a clear, yellow oil.

${ }^{1} \mathrm{H}$ NMR $\left(\mathrm{CDCl}_{3}, 500 \mathrm{MHz}\right) \delta 1.36(\mathrm{~s}, 9 \mathrm{H}), 7.47$ (td, $J=7.7,1.2$ $\mathrm{Hz}, 1 \mathrm{H}), 7.55$ (td, $J=7.7,1.5 \mathrm{~Hz}, 1 \mathrm{H}), 7.69$ (ddd, $J=7.6,1.3,0.6$ $\mathrm{Hz}, 1 \mathrm{H}), 7.73(\mathrm{ddd}, J=7.6,1.5,0.6 \mathrm{~Hz}, 1 \mathrm{H}) .{ }^{13} \mathrm{C}$ NMR $\left(\mathrm{CDCl}_{3}, 125 \mathrm{MHz}\right) \delta 31.3\left(\mathrm{CH}_{3}\right), 49.2$ (C), $118.5(\mathrm{C}), 121.6(\mathrm{C}), 129.5(\mathrm{CH}), 132.5(\mathrm{CH}), 134.0(\mathrm{CH}), 136.9(\mathrm{C}), 139.2(\mathrm{CH})$. GC-MS (EI) $191\left([\mathrm{M}]^{+}, 20 \%\right), 176(4 \%), 135(100 \%), 108(6 \%), 90$ (7\%), 57 (65\%). FT-IR $\left(\mathrm{cm}^{-1}\right.$, neat, ATR) 3062 (vw), 2963 (w), 2228 (w), 1485 (w), 1365 (w), 1164 (m), 761 (vs). HRMS (ES+) calcd for $\mathrm{C}_{11} \mathrm{H}_{13} \mathrm{NSNa}[\mathrm{M}+\mathrm{Na}]^{+}: 214.0666$, found: 214.0658 .

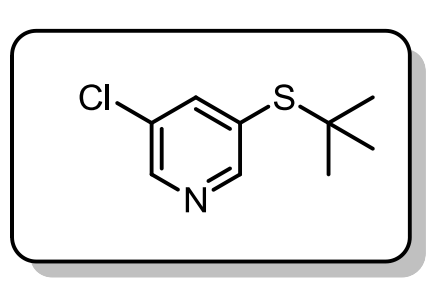

3-(tert-Butylthio)-5-chloropyridine, $\mathbf{3 y}(61 \mathrm{mg}, 61 \%)$ was prepared according to the general procedure for thioetherification from 3bromo-5-chloropyridine $(96 \mathrm{mg}, 0.5 \mathrm{mmol})$ and tert-butylthiol $(67.6$ $\mu \mathrm{L}, 54 \mathrm{mg}, 0.60 \mathrm{mmol})$. The desired thioether was obtained as a clear, colorless oil.

${ }^{1} \mathrm{H}$ NMR $\left(\mathrm{CDCl}_{3}, 500 \mathrm{MHz}\right) \delta 1.31(\mathrm{~s}, 9 \mathrm{H}), 7.84(\mathrm{dd}, J=2.4,1.8$ $\mathrm{Hz}, 1 \mathrm{H}), 8.56(\mathrm{~d}, J=2.4 \mathrm{~Hz}, 1 \mathrm{H}), 8.58(\mathrm{~d}, J=1.8 \mathrm{~Hz}, 1 \mathrm{H}) .{ }^{13} \mathrm{C}$ NMR $\left(\mathrm{CDCl}_{3}, 125 \mathrm{MHz}\right) \delta 31.2$ $\left(\mathrm{CH}_{3}\right), 47.3(\mathrm{C}), 131.3(\mathrm{C}), 131.7(\mathrm{C}), 143.9(\mathrm{CH}), 148.8(\mathrm{CH}), 154.7(\mathrm{CH}) . \mathrm{GC}-\mathrm{MS}$ (EI) 203 ([M] $\left.{ }^{+},{ }^{\mathrm{c}} 8 \%\right), 201\left([\mathrm{M}]^{+},{ }^{35} \mathrm{Cl} 21 \%\right), 147\left({ }^{37} \mathrm{Cl} 34 \%\right), 145\left({ }^{35} \mathrm{Cl} 90 \%\right), 117$ (7\%), 101 (5\%), 82(9\%), $73(12 \%), 57(100 \%)$. FT-IR $\left(\mathrm{cm}^{-1}\right.$, neat, ATR) $3042(\mathrm{vw}), 2962(\mathrm{w}), 1553(\mathrm{w}), 1398$ (m), $1365(\mathrm{~m}), 1167(\mathrm{~m}), 1102(\mathrm{~m}), 1020(\mathrm{~m}), 882(\mathrm{~m}), 812(\mathrm{~m}), 700$ (s). HRMS (CI+) calcd for $\mathrm{C}_{9} \mathrm{H}_{12} \mathrm{ClNS}[\mathrm{M}]^{+}: 201.0379$, found: 201.0386.

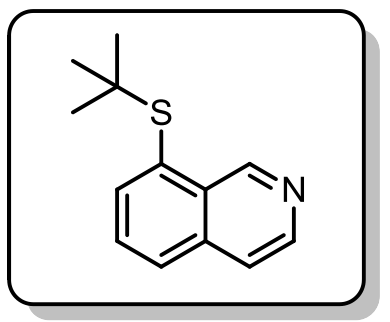

8-(tert-Butylthio)isoquinoline, $\mathbf{3 z}(80 \mathrm{mg}, \quad 73 \%)$ was prepared according to the general procedure for thioetherification from aryl halide $(104 \mathrm{mg}, 0.5 \mathrm{mmol})$ and tert-butylthiol $(67.6 \mu \mathrm{L}, 54 \mathrm{mg}, 0.60$ $\mathrm{mmol})$. The desired thioether was obtained as a clear, pale yellow oil.

${ }^{1} \mathrm{H}$ NMR $\left(\mathrm{CDCl}_{3}, 500 \mathrm{MHz}\right) \delta 1.32(\mathrm{~s}, 9 \mathrm{H}), 7.64-7.70(\mathrm{~m}, 2 \mathrm{H}), 7.87$ $(\mathrm{dd}, J=7.0,6.1 \mathrm{~Hz}, 2 \mathrm{H}), 8.57(\mathrm{~d}, J=5.5 \mathrm{~Hz}, 1 \mathrm{H}), 10.08(\mathrm{~s}, 1 \mathrm{H}) .{ }^{13} \mathrm{C}$ NMR $\left(\mathrm{CDCl}_{3}, 125 \mathrm{MHz}\right) \delta 31.5\left(\mathrm{CH}_{3}\right), 48.1(\mathrm{C}), 120.5(\mathrm{CH}), 128.4$ $(\mathrm{CH}), 129.9(\mathrm{CH}), 131.5(\mathrm{C}), 132.1(\mathrm{C}), 136.9(\mathrm{C}), 138.8(\mathrm{CH}), 143.4(\mathrm{CH}), 152.6(\mathrm{CH})$. GCMS (EI) $217\left([\mathrm{M}]^{+}, 11 \%\right), 161(100 \%), 134(6 \%), 117(12 \%), 89(7 \%), 57(11 \%)$. FT-IR $\left(\mathrm{cm}^{-1}\right.$, neat, ATR) 3049 (vw), 2961 (w), 1611 (w), 1363 (w), 1155 (m), 974 (w), 833 (vs), 753 (w), 652 (w). HRMS (ES+) calcd for $\mathrm{C}_{13} \mathrm{H}_{16} \mathrm{NS}[\mathrm{M}+\mathrm{H}]^{+}: 218.1003$, found: 218.0997. 


\section{Representative Procedure for Large Scale Thioetherification}

\section{Using silicate 1a:}

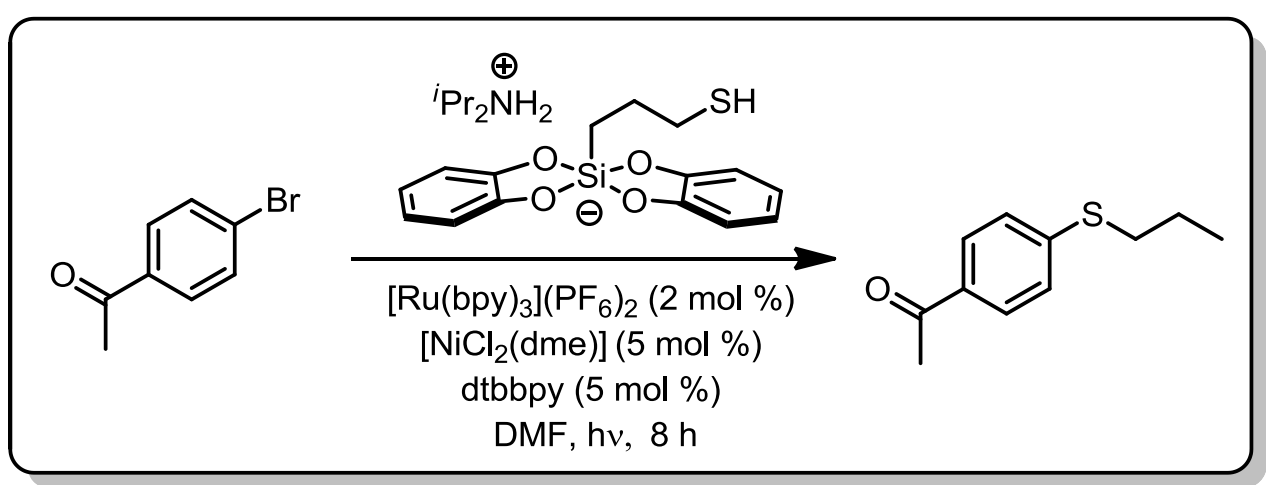

\section{1-(4-(Propylthio)phenyl)ethanone (2d)}

To an oven dried, $100 \mathrm{~mL}$ round bottom flask equipped with an appropriately sized stir bar were added alkylthiolsilicate $1 \mathbf{a}(2.53 \mathrm{~g}, 6 \mathrm{mmol}, 1.2 \mathrm{equiv})$, [ $\left.\mathrm{NiCl}_{2}(\mathrm{dme})\right]$ (55 mg, $0.250 \mathrm{mmol}, 0.025$ equiv), dtbbpy (67.1 mg, $0.250 \mathrm{mmol}, 0.025$ equiv), and [Ru(bpy) $\left.{ }_{3}\right]\left(\mathrm{PF}_{6}\right)(86.0 \mathrm{mg}, 0.100 \mathrm{mmol}$, 0.02 equiv). The flask was sealed with rubber a septum and was evacuated three times via its inlet valve and purged with argon. The flask was then charged with the 4-bromoacetophenone (0.995 g, $5 \mathrm{mmol}, 1$ equiv) dissolved in anhyd, degassed DMF (50 mL) via a syringe. The now bright red solution was irradiated in the aforementioned LED reactor. The temperature of the reaction was maintained at approximately $27{ }^{\circ} \mathrm{C}$ via a fan. The solution was stirred vigorously while being irradiated. Reaction progress was monitored by GC/MS. Once complete $(\sim 8 \mathrm{~h})$, the now opaque, milky-brown solution was transferred to a separatory funnel and diluted with deionized $\mathrm{H}_{2} \mathrm{O}(\sim 150 \mathrm{~mL})$ and $\mathrm{Et}_{2} \mathrm{O}(\sim 100 \mathrm{~mL})$. The layers were separated, and the aqueous layer was extracted with $\mathrm{Et}_{2} \mathrm{O}(3 \times \sim 100 \mathrm{~mL})$. The combined organic layers were washed with 2 $\mathrm{M} \mathrm{NaOH}(2 \times \sim 100 \mathrm{~mL}), 2 \mathrm{M} \mathrm{HCl}(\sim 100 \mathrm{~mL})$, deionized $\mathrm{H}_{2} \mathrm{O}(\sim 100 \mathrm{~mL})$, and brine $(\sim 100 \mathrm{~mL})$. The organic layer was dried $\left(\mathrm{Na}_{2} \mathrm{SO}_{4}\right)$, and the solvent was removed in vacuo by rotary evaporation. Further purification was accomplished by $\mathrm{SiO}_{2}$ column chromatography (gradient hexane to 9:1 hexane/EtOAc) to give the desired thioether $\mathbf{2 d}(0.748 \mathrm{~g}, 77 \%)$. as a clear, colorless oil that solidified upon standing to a white, waxy solid $\left(\mathrm{mp}=36^{\circ} \mathrm{C}\right)$.

${ }^{1} \mathrm{H}$ NMR $\left(\mathrm{CDCl}_{3}, 500 \mathrm{MHz}\right) \delta 1.06(\mathrm{t}, J=7.3 \mathrm{~Hz}, 3 \mathrm{H}), 1.69-1.77(\mathrm{~m}, 2 \mathrm{H}), 2.55-2.56(\mathrm{~m}, 3 \mathrm{H})$, $2.97(\mathrm{t}, J=7.3 \mathrm{~Hz}, 2 \mathrm{H}), 7.30(\mathrm{~d}, J=8.2 \mathrm{~Hz}, 2 \mathrm{H}), 7.84(\mathrm{~d}, J=8.2 \mathrm{~Hz}, 2 \mathrm{H}) .{ }^{13} \mathrm{C} \mathrm{NMR}\left(\mathrm{CDCl}_{3}\right.$, $125 \mathrm{MHz}) \delta 13.8\left(\mathrm{CH}_{3}\right), 22.5\left(\mathrm{CH}_{2}\right), 26.7\left(\mathrm{CH}_{3}\right), 34.3\left(\mathrm{CH}_{2}\right), 126.6(\mathrm{CH}), 129.0(\mathrm{CH}), 134.1(\mathrm{C})$, 145.2 (C), 197.4 (C). GC-MS (EI) 194 ([M] $\left.{ }^{+}, 88 \%\right), 179$ (100\%), 137 (71\%), 123 (13\%), 109 (20\%), 69 (6\%). FT-IR (cm ${ }^{-1}$, neat, ATR) 2967 (w), 1673 (vs), 1587 (s) 1356 (s), 1264 (s), 1180 (s), 1096 (s), 956 (s), 813 (vs), 745 (m), 586 (s). HRMS (CI+) calcd for $\mathrm{C}_{11} \mathrm{H}_{14} \mathrm{OS}[\mathrm{M}]^{+}$: 194.0765, found: 194.0762. 


\section{Using thiols with silicate $1 \mathrm{~b}$ as a hydride abstractor}

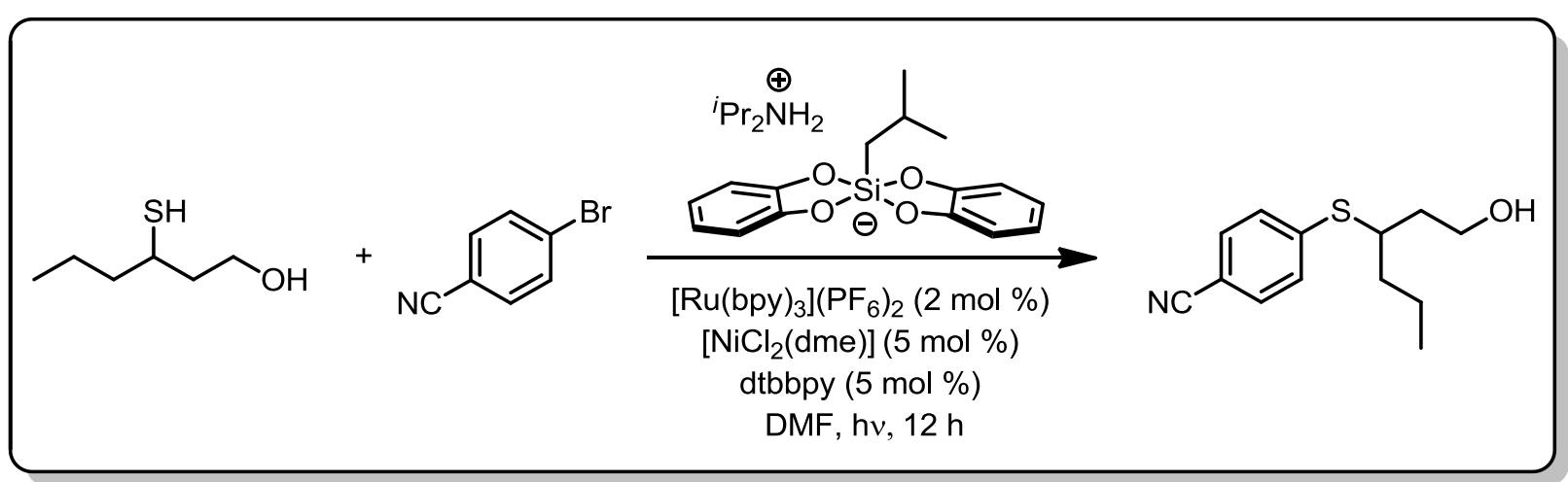

\section{4-((1-Hydroxyhexan-3-yl)thio)benzonitrile (3g)}

To a $100 \mathrm{~mL}$ round bottom flask equipped with an appropriately sized stir bar were added added alkylsilicate $1 \mathrm{~b}$ (3.03 g, $7.5 \mathrm{mmol}, 1.5$ equiv), [ $\left.\mathrm{NiCl}_{2}(\mathrm{dme})\right]$ (55 mg, $0.250 \mathrm{mmol}, 0.025$ equiv), dtbbpy (67.1mg, $0.250 \mathrm{mmol}, 0.025$ equiv), and $\left[\mathrm{Ru}(\mathrm{bpy})_{3}\right]\left(\mathrm{PF}_{6}\right)(86.0 \mathrm{mg}, 0.100 \mathrm{mmol}, 0.02$ equiv). The flask was sealed with rubber septum and was evacuated three times via its inlet valve and purged with argon. The flask was then charged with the 4-bromobenzonitrile $(0.910 \mathrm{~g}, 5$ mmol, 1 equiv) and 3-mercaptohexan-1-ol (0.805 g, $6 \mathrm{mmol}, 1.2 \mathrm{gequiv})$ dissolved in anhyd, degassed DMF $(50 \mathrm{~mL})$ via a syringe. The now bright red solution was irradiated in the aforementioned LED reactor. The temperature of the reaction was maintained at approximately $27{ }^{\circ} \mathrm{C}$ via a fan. The solution was stirred vigorously while being irradiated. Reaction progress was monitored by GC/MS. Once complete $(\sim 12 \mathrm{~h})$, the now opaque milky-brown solution was transferred to a separatory funnel an diluted with deionized $\mathrm{H}_{2} \mathrm{O}(\sim 150 \mathrm{~mL})$ and $\mathrm{Et}_{2} \mathrm{O}(\sim 100$ $\mathrm{mL})$. The layers were separated, and the aqueous layer was extracted with $\mathrm{Et}_{2} \mathrm{O}(3 \times \sim 100 \mathrm{~mL})$. The combined organic layers were washed with $2 \mathrm{M} \mathrm{NaOH}(2 \times \sim 100 \mathrm{~mL}), 2 \mathrm{M} \mathrm{HCl}(\sim 100 \mathrm{~mL})$, deionized $\mathrm{H}_{2} \mathrm{O}(\sim 100 \mathrm{~mL})$, and brine $(\sim 100 \mathrm{~mL})$. The organic layer was dried $\left(\mathrm{Na}_{2} \mathrm{SO}_{4}\right)$, and the solvent was removed in vacuo by rotary evaporation. Further purification was accomplished by $\mathrm{SiO}_{2}$ column chromatography (gradient hexane to 9:1 hexane:EtOAc). to give the desired thioether $\mathbf{3 g}(1.01 \mathrm{~g}, 86 \%)$ as a thick yellow oil.

${ }^{1} \mathrm{H}$ NMR $\left(\mathrm{CDCl}_{3}, 500 \mathrm{MHz}\right) \delta 0.91(\mathrm{t}, J=7.2 \mathrm{~Hz}, 3 \mathrm{H}), 1.41-1.56(\mathrm{~m}, 2 \mathrm{H}), 1.57-1.72(\mathrm{~m}, 3 \mathrm{H})$, 1.82 (ddt, $J=14.2,8.3,5.7 \mathrm{~Hz}, 1 \mathrm{H}), 1.94$ (ddt, $J=14.3,8.3,5.7 \mathrm{~Hz} 1 \mathrm{H}$ ), 3.44-3.53 (m, 1H), $3.80(\mathrm{qd}, J=11.3,6.4 \mathrm{~Hz}, 1 \mathrm{H}), 7.39(\mathrm{~d}, J=8.2 \mathrm{~Hz}, 2 \mathrm{H}), 7.51(\mathrm{~d}, J=8.2 \mathrm{~Hz}, 2 \mathrm{H}) .{ }^{13} \mathrm{C} \mathrm{NMR}$ $\left(\mathrm{CDCl}_{3}, 125 \mathrm{MHz}\right) \delta 14.2\left(\mathrm{CH}_{3}\right), 20.2\left(\mathrm{CH}_{2}\right), 37.4\left(\mathrm{CH}_{2}\right), 37.6\left(\mathrm{CH}_{2}\right), 44.4(\mathrm{CH}), 60.3\left(\mathrm{CH}_{2}\right)$, $108.8(\mathrm{C}), 119.1(\mathrm{C}), 129.0(\mathrm{CH}), 132.5(\mathrm{CH}), 144.7(\mathrm{C})$. GC-MS (EI) $235\left([\mathrm{M}]^{+}, 4 \%\right), 207$ (17\%), 135 (10\%), 125 (100\%), 83 (15\%), 55 (24\%). FT-IR ( $\mathrm{cm}^{-1}$, neat, ATR) 3434 (m, br), 2957 (m), 2931 (m), 2226 (s), 1591 (vs), 1485 (s), 1086 (vs), 1048 (s), 820 (vs), 543 (vs). HRMS (ES+) calcd for $\mathrm{C}_{13} \mathrm{H}_{18} \mathrm{NOS}[\mathrm{M}+\mathrm{H}]^{+}: 236.1109$, found: 236.1105. 


\section{Control and Mechanistic Studies}

\section{Procedure for control studies using alkylthiolsilicate 1a}

To a $4 \mathrm{~mL}$ reaction vial equipped with an appropriately sized stir bar, was added alkylthiolsilicate 1a (44 mg, $0.105 \mathrm{mmol}, 1.05$ equiv) and 4-bromoacetophenone (20 mg, 0.60 mmol, 1 equiv). The vial was sealed with a cap containing a TFE lined silicone septa and was evacuated three times via an inlet needle then purged with argon. The vial was then charged with $\mathrm{NiCl}_{2} \bullet$ dme (1.1 mg, $0.025 \mathrm{mmol}, 0.05$ equiv), dtbbpy (1.3 mg, 0.025, 0.05 equiv), and $\mathrm{Ru}(\mathrm{bpy})_{3}\left(\mathrm{PF}_{6}\right)(1.8 \mathrm{mg}, 0.01 \mathrm{mmol}, 0.02$ equiv) dissolved in anhyd, degassed DMF (1 mL) via a syringe. The cap was sealed with Parafilm ${ }^{\circledR}$ and the now bright red solution was irradiated in the aforementioned LED reactor for $12 \mathrm{~h}$. The temperature of the reaction was maintained at approximately $27{ }^{\circ} \mathrm{C}$ via a fan. After $12 \mathrm{~h}$, reaction progress was evaluated by GC/MS.

Table S1: Control Studies using Alkylthiolsilicate $1 \mathrm{a}^{a}$

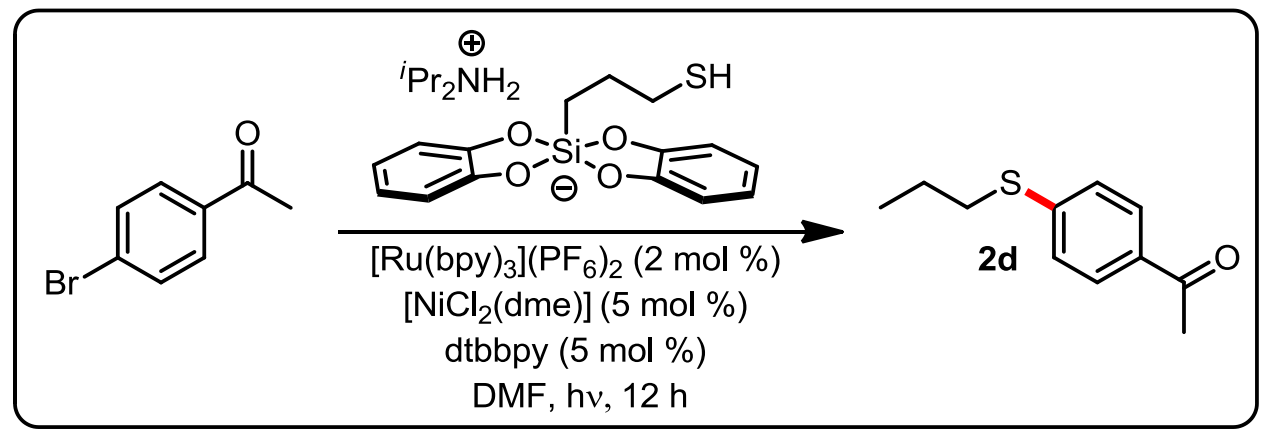

\begin{tabular}{|c|c|c|}
\hline Entry & Deviation from procedure & Conversion to $2 \mathrm{~d}$ \\
\hline 1 & None & $100 \%$ \\
\hline 2 & No photocatalyst $\left(\left[R u(b p y)_{3}\right]\left(P F_{6}\right)_{2}\right)$ & $0 \%$ \\
\hline 3 & No light & $0 \%$ \\
\hline 4 & $\mathrm{No}\left[\mathrm{NiCl}_{2}(\mathrm{dme})\right]$ & $0 \%$ \\
\hline 5 & No ligand (dtbbpy) & $57 \%$ \\
\hline
\end{tabular}

${ }^{a}$ Percentages determined by area/(total area of 4-bromoacetophenone and 2d) of GC/MS trace of run.

\section{Procedure for evaluating effect of alkylsilicate structure on photoredox thioetherifcation}

To a $4 \mathrm{~mL}$ reaction vial equipped with an appropriately sized stir bar, was added the appropriate alkylsilicate ( $0.15 \mathrm{mmol}, 1.5$ equiv) and 4-bromobenzonitrile (18.2 $\mathrm{mg}, 0.10 \mathrm{mmol}$, 1 equiv). The vial was sealed with a cap containing a TFE lined silicone septa and was evacuated three times via an inlet needle then purged with argon. The vial was then charged $\left[\mathrm{NiCl}_{2}(\mathrm{dme})\right](1.1$ $\mathrm{mg}, 0.005 \mathrm{mmol}, 0.05$ equiv), dtbbpy $\left(1.3 \mathrm{mg}, 0.005,0.05\right.$ equiv), and $\left[\mathrm{Ru}(\mathrm{bpy})_{3}\right]\left(\mathrm{PF}_{6}\right)(1.8 \mathrm{mg}$, $0.002 \mathrm{mmol}, 0.02$ equiv) all dissolved in anhyd, degassed DMF (1 mL) via a syringe. This was followed by rapid addition of cyclohexanethiol $(14.7 \mu \mathrm{L}, 13.9 \mathrm{mg}, 0.120 \mathrm{mmol}, 1.2$ equiv) via a 
$\mu \mathrm{L}$ syringe. The cap was sealed with Parafilm ${ }^{\circledR}$ and the now bright red solution was irradiated in the aforementioned LED reactor for $36 \mathrm{~h}$. The temperature of the reaction was maintained at approximately $27^{\circ} \mathrm{C}$ via a fan. After $36 \mathrm{~h}$, reaction progress was evaluated by GC/MS.

Table S2: Effect of Alkylsilicate Structure on the Outcome of Photoredox Thioetherification ${ }^{a}$

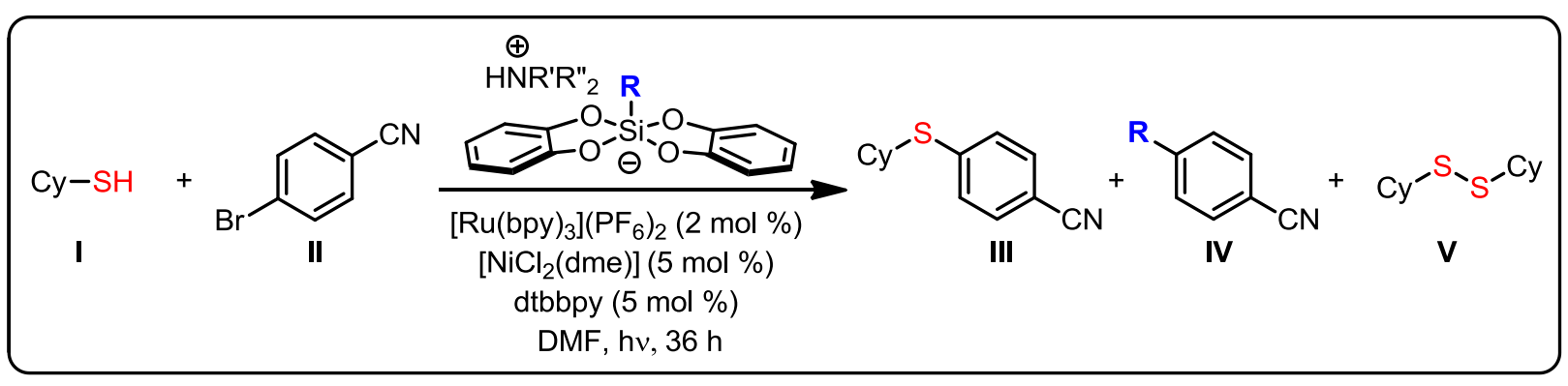

\begin{tabular}{|c|c|c|c|c|c|c|}
\hline Entry & Alkylsilicate & I & II & III & IV & $\mathbf{V}$ \\
\hline 1 & & $6 \%$ & $69 \%$ & $22 \%$ & $0 \%$ & $3 \%$ \\
\hline 2 & & trace & $48 \%$ & $46 \%$ & $0 \%$ & $6 \%$ \\
\hline 3 & & trace & $3 \%$ & $12 \%$ & $85 \%$ & trace \\
\hline 4 & & trace & $0 \%$ & $99 \%$ & $0 \%$ & $1 \%$ \\
\hline 5 & & $0 \%$ & $0 \%$ & $98 \%$ & $0 \%$ & $2 \%$ \\
\hline 7 & $\mathrm{Et}_{3} \mathrm{NH} \quad 1 \mathrm{a}$ & $0 \%$ & $0 \%$ & $99 \%$ & $0 \%$ & $1 \%$ \\
\hline 8 & None & $34 \%$ & $62 \%$ & $0 \%$ & $0 \%$ & $4 \%$ \\
\hline
\end{tabular}

${ }^{a}$ Percentages determined by area/(total area of mixture components) of GC/MS trace of run. 


\section{Procedure for evaluating effect of alkylsilicate structure on photoredox thioetherifcation}

To a $4 \mathrm{~mL}$ reaction vial equipped with an appropriately sized stir bar, was added the appropriate alkylsilicate $1 \mathbf{b}$ (61 mg, $0.15 \mathrm{mmol}, 1.5$ equiv) and 4-bromoacetophenone (20 mg, $0.10 \mathrm{mmol}, 1$ equiv). The vial was sealed with a cap containing a TFE lined silicone septa and was evacuated three times via an inlet needle then purged with argon. The vial was then charged $\left[\mathrm{NiCl}_{2}(\mathrm{dme})\right]$ (1.1 mg, $0.005 \mathrm{mmol}, 0.05$ equiv), dtbbpy ( $1.3 \mathrm{mg}, 0.005,0.05$ equiv), and $\left[\mathrm{Ru}(\mathrm{bpy})_{3}\right]\left(\mathrm{PF}_{6}\right)(1.8$ $\mathrm{mg}, 0.002 \mathrm{mmol}, 0.02$ equiv) all dissolved in anhyd, degassed DMF (1 mL) via a syringe. This was followed by rapid addition of cyclohexanethiol $(14.7 \mu \mathrm{L}, 13.9 \mathrm{mg}, 0.120 \mathrm{mmol}, 1.2$ equiv) via a $\mu \mathrm{L}$ syringe. The cap was sealed with Parafilm ${ }^{\circledR}$ and the now bright red solution was irradiated in the aforementioned LED reactor for $24 \mathrm{~h}$. The temperature of the reaction was maintained at approximately $27{ }^{\circ} \mathrm{C}$ via a fan. After $24 \mathrm{~h}$, reaction progress was evaluated by GC/MS and tabulated.

Table S3: Control Studies using Thiols and Alkylthiolsilicate $\mathbf{1 b}^{\mathrm{a}}$

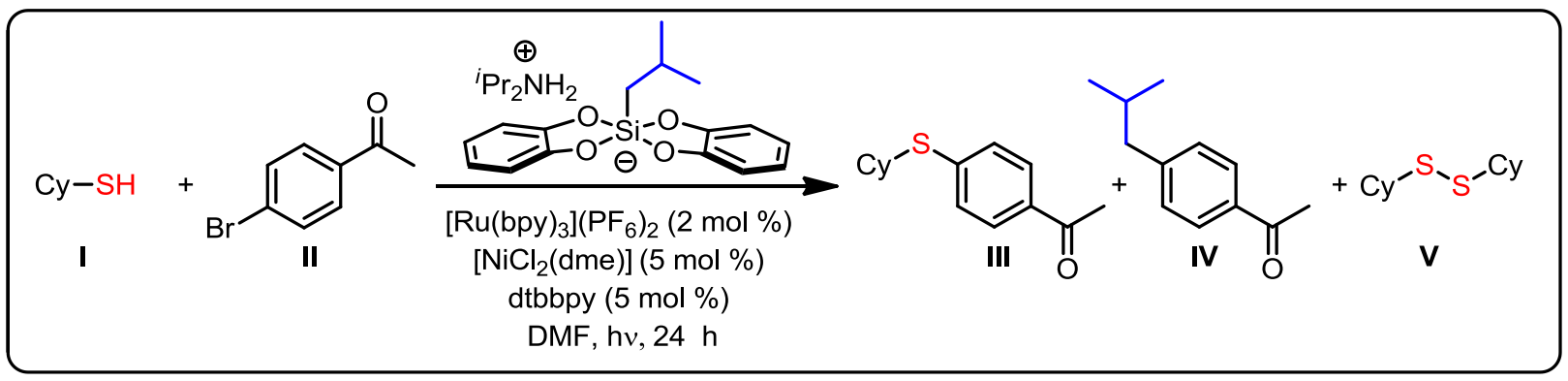

\begin{tabular}{|c|c|c|c|c|c|c|}
\hline Entry & Deviation from procedure & I & II & III & IV & $\mathbf{V}$ \\
\hline 1 & None & $6 \%$ & $0 \%$ & $92 \%$ & $0 \%$ & $2 \%$ \\
\hline 2 & No photocatalyst $\left(\left[R u(b p y)_{3}\right]\left(P F_{6}\right)_{2}\right)$ & $33 \%$ & $64 \%$ & $0 \%$ & $0 \%$ & $3 \%$ \\
\hline 3 & $\mathrm{No}\left[\mathrm{NiCl}_{2}(\mathrm{dme})\right]$ & $28 \%$ & $66 \%$ & $0 \%$ & $0 \%$ & $6 \%$ \\
\hline 4 & No ligand (dtbbpy) & $14 \%$ & $60 \%$ & $0 \%$ & $0 \%$ & $26 \%$ \\
\hline 5 & No light & $34 \%$ & $63 \%$ & $0 \%$ & $0 \%$ & $3 \%$ \\
\hline 6 & No alkylsilicate & $34 \%$ & $62 \%$ & $0 \%$ & $0 \%$ & $4 \%$ \\
\hline 7 & No cyclohexanethiol & $0 \%$ & $0 \%$ & $0 \%$ & $100 \%$ & $0 \%$ \\
\hline
\end{tabular}

${ }^{a}$ Percentages determined by area/(total area of mixture components) of GC/MS trace of run. 


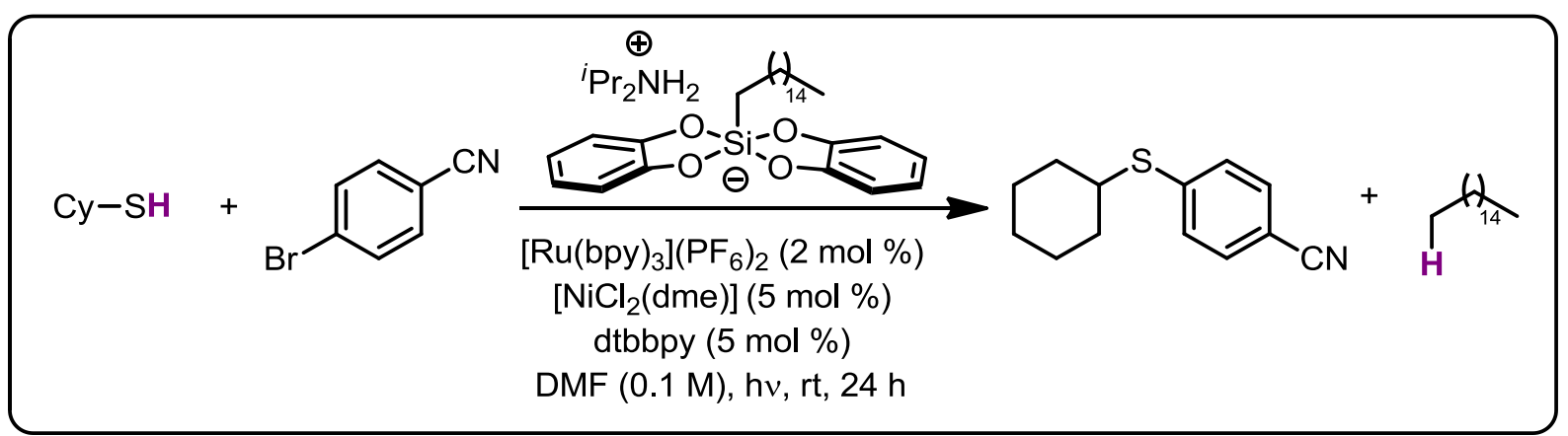

\section{Determination of fate of alkyl component using hexadecylsilicate 1c}

To a $4 \mathrm{~mL}$ reaction vial equipped with an appropriately sized stir bar, was added the appropriate alkylsilicate $1 \mathrm{~g}$ (60 mg, $0.105 \mathrm{mmol}, 1.05$ equiv) and 4-bromoacetophenone $(20 \mathrm{mg}, 0.10 \mathrm{mmol}$, 1 equiv). The vial was sealed with a cap containing a TFE lined silicone septa and was evacuated three times via an inlet needle then purged with argon. The vial was then charged with cyclohexanethiol $\left(14.7 \mu \mathrm{L}, 13.9 \mathrm{mg}, 0.120 \mathrm{mmol}, 1.2\right.$ equiv) followed by $\left[\mathrm{NiCl}_{2}(\mathrm{dme})\right](1.1 \mathrm{mg}$, $0.005 \mathrm{mmol}, 0.05$ equiv), dtbbpy ( $1.3 \mathrm{mg}, 0.005,0.05$ equiv), and $\left[\mathrm{Ru}(\mathrm{bpy})_{3}\right]\left(\mathrm{PF}_{6}\right)(1.8 \mathrm{mg}$, $0.002 \mathrm{mmol}, 0.02$ equiv) all dissolved in anhyd, degassed DMF $(1 \mathrm{~mL})$ via a syringe. The cap was sealed with Parafilm and the now bright red solution was irradiated in the aforementioned LED reactor for $24 \mathrm{~h}$. The temperature of the reaction was maintained at approximately $27{ }^{\circ} \mathrm{C}$ via a fan. Reaction progress was monitored by GC/MS and hexadecane was found to form in approximately equal quantity to the expected thioether over time. 


\section{${ }^{1}$ H NMR Spectra of Synthesized Compounds}

diisopropylammonium bis(catecholato)(3-mercaptopropyl)silicate $500 \mathrm{MHz}, \mathrm{MeCN}-\mathrm{d} 3$
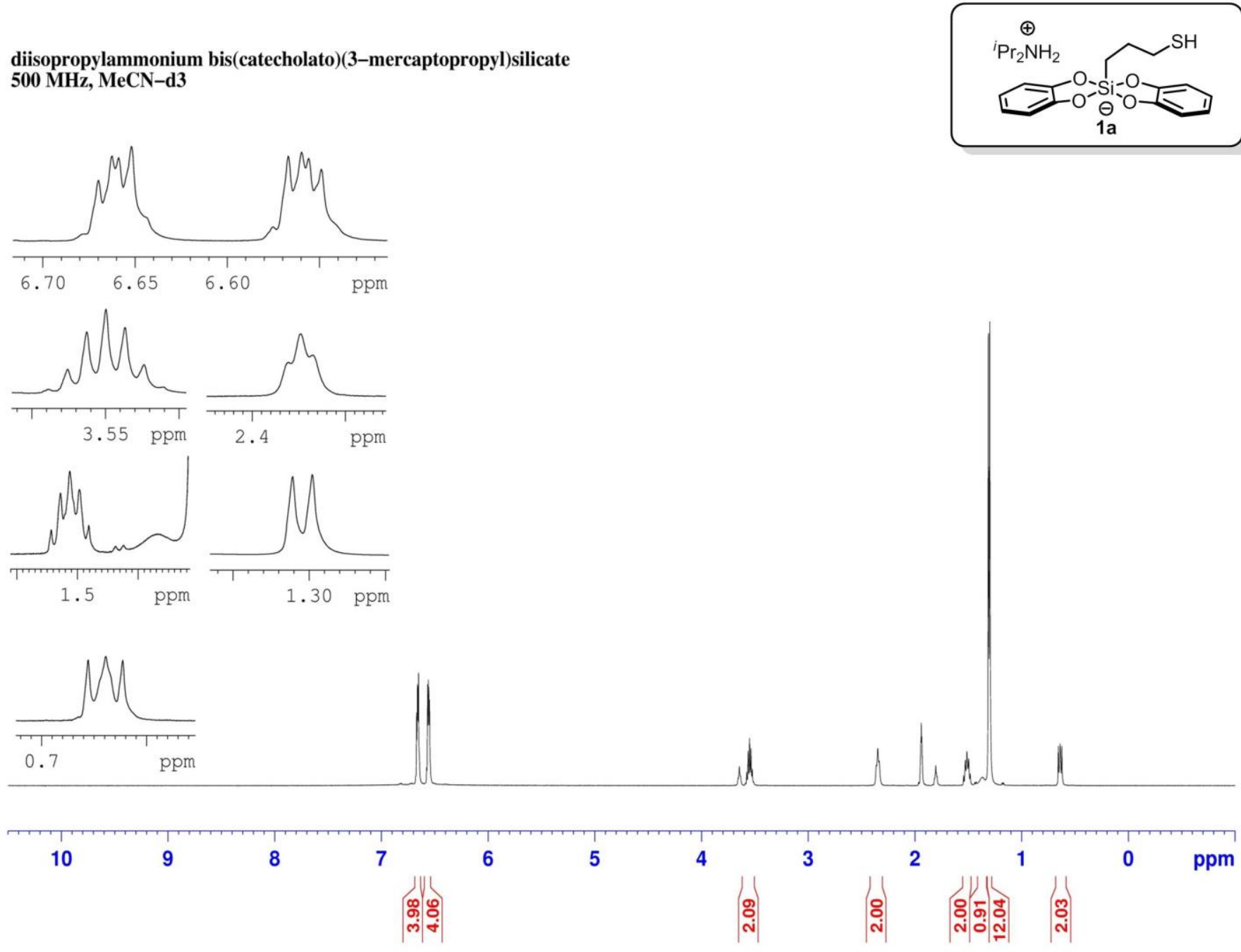
diisopropylammonium bis(catecholato)isobutylsilicate 500 MHz, DMSO-d6
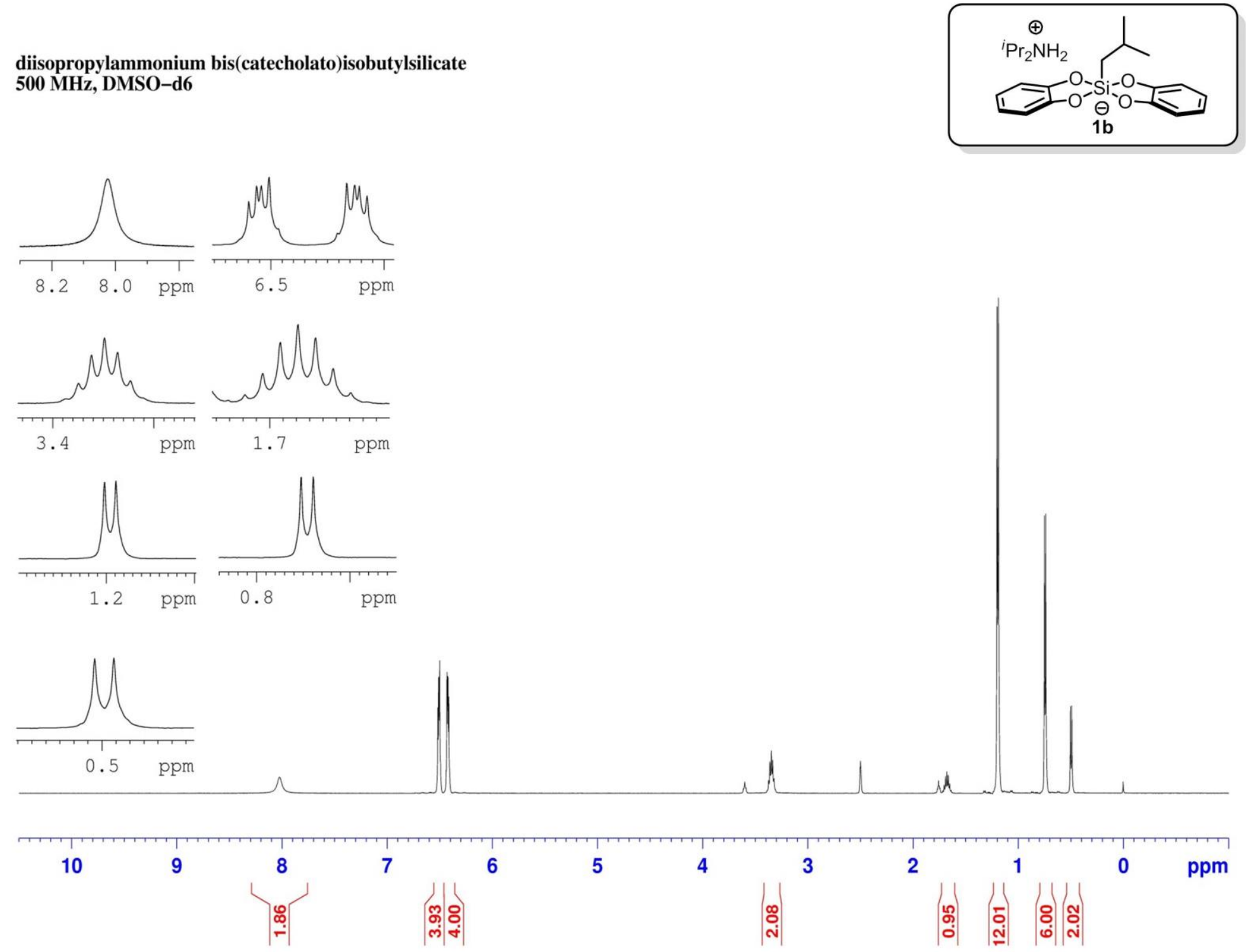
diisopropylammonium Bis(catecholato)hexadecylsilicate 500 MHz, DMSO-d6
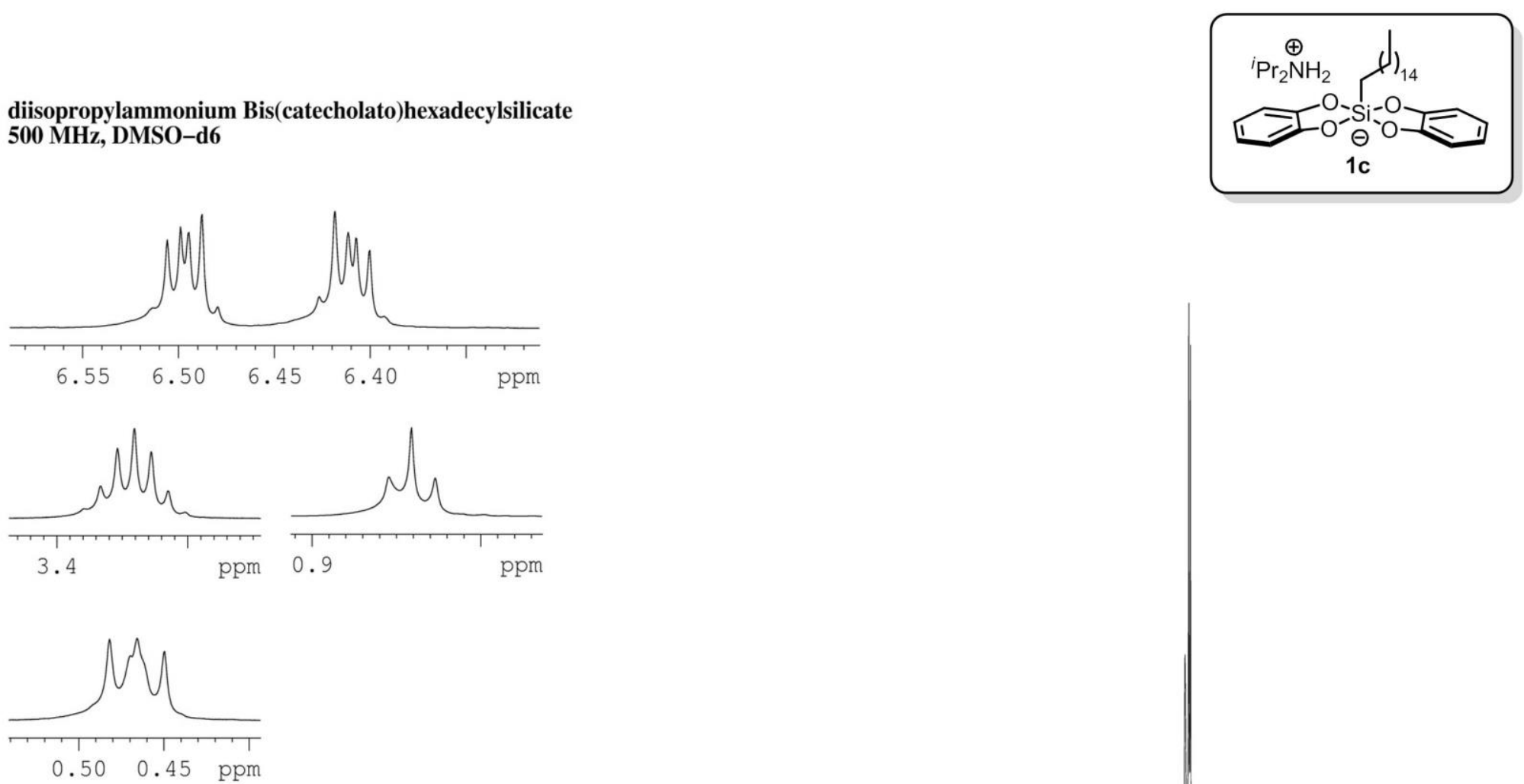
[1,1'-biphenyl]-4-yl(propyl)sulfane $500 \mathrm{MHz}, \mathrm{CDCl} 3$
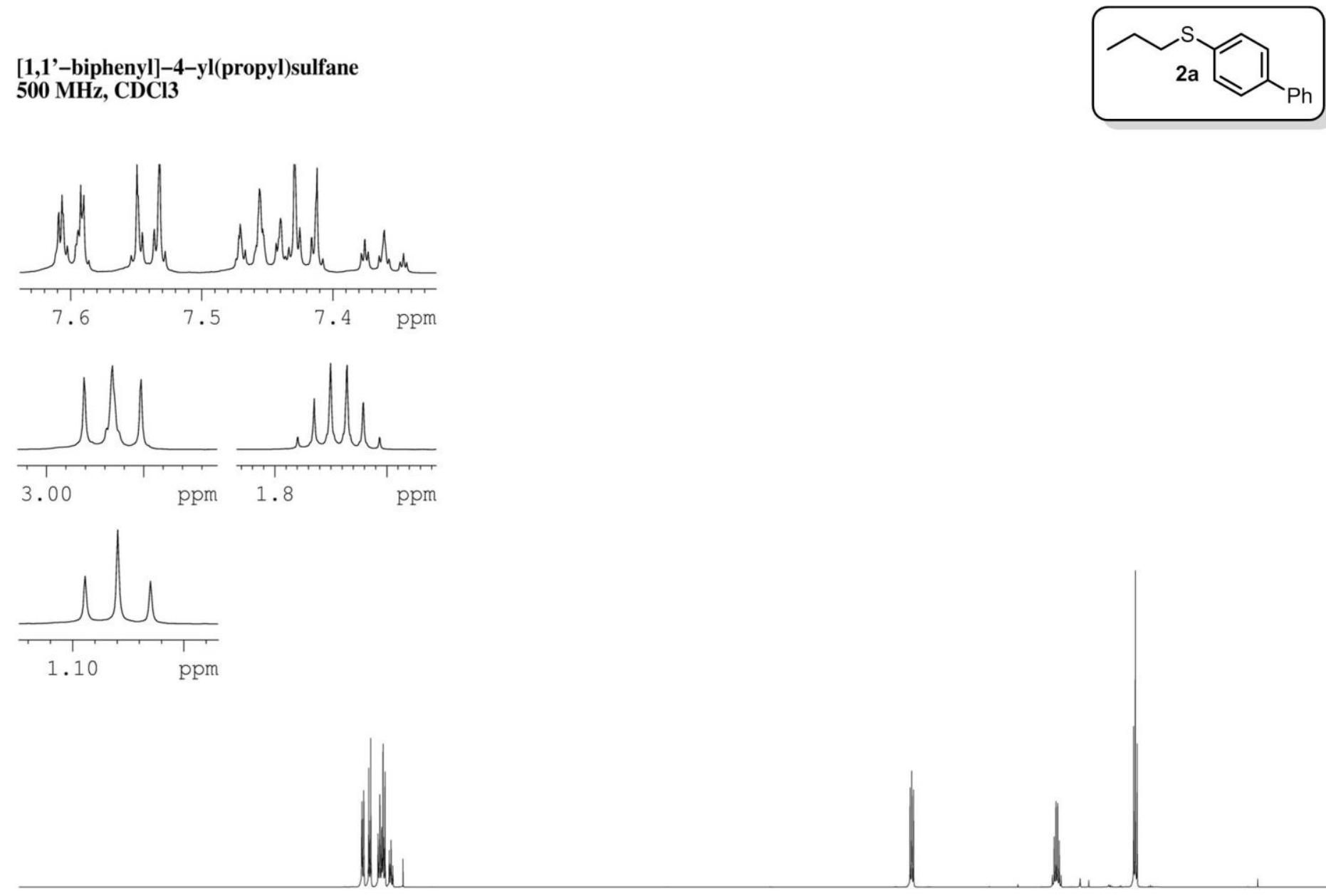

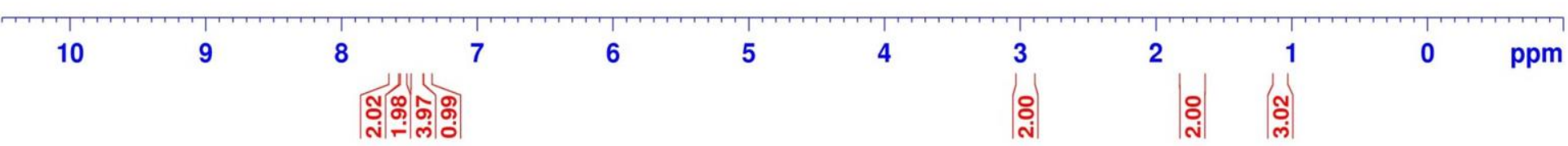


(4-benzylphenyl)(propyl)sulfane
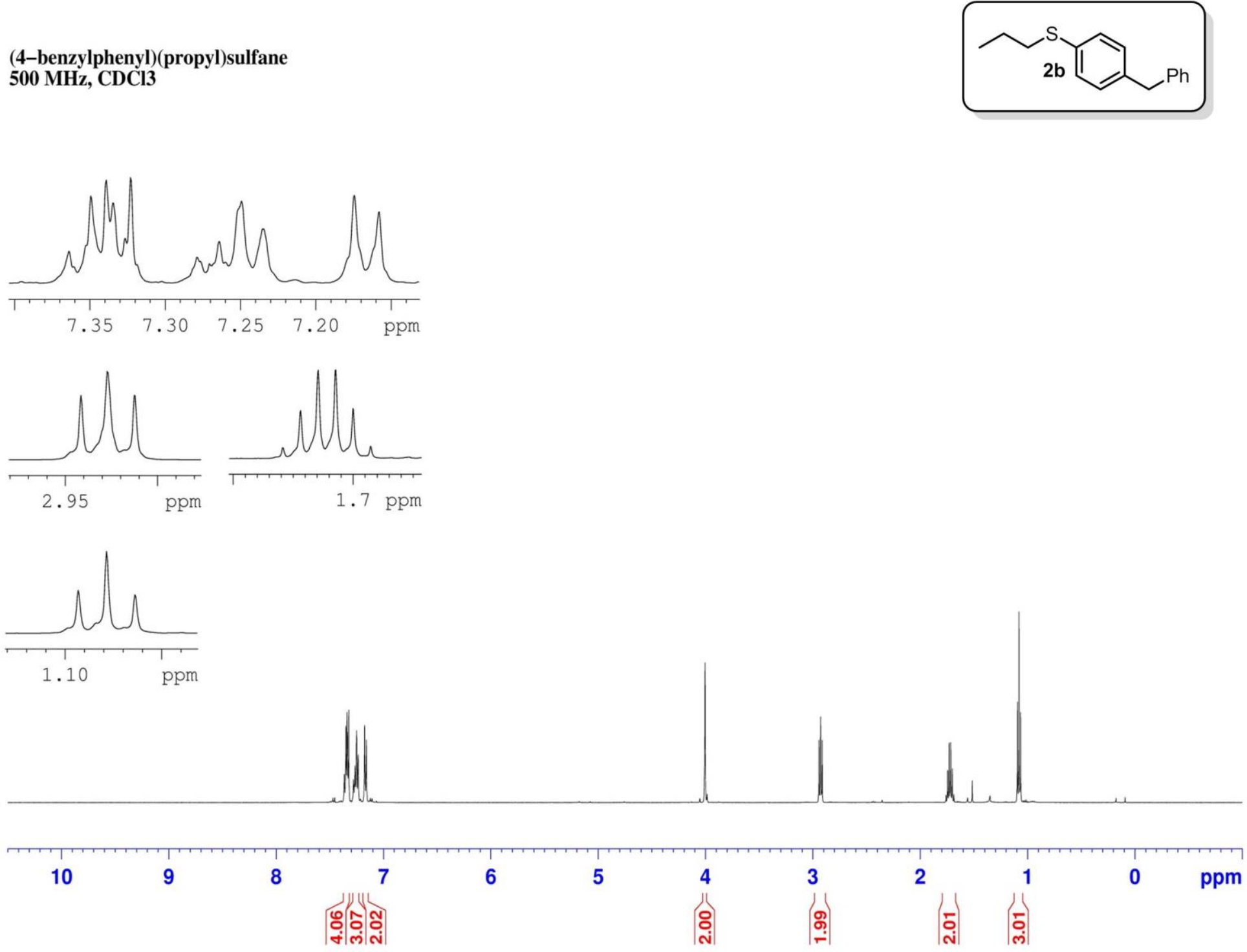
4-(propylthio)benzonitrile
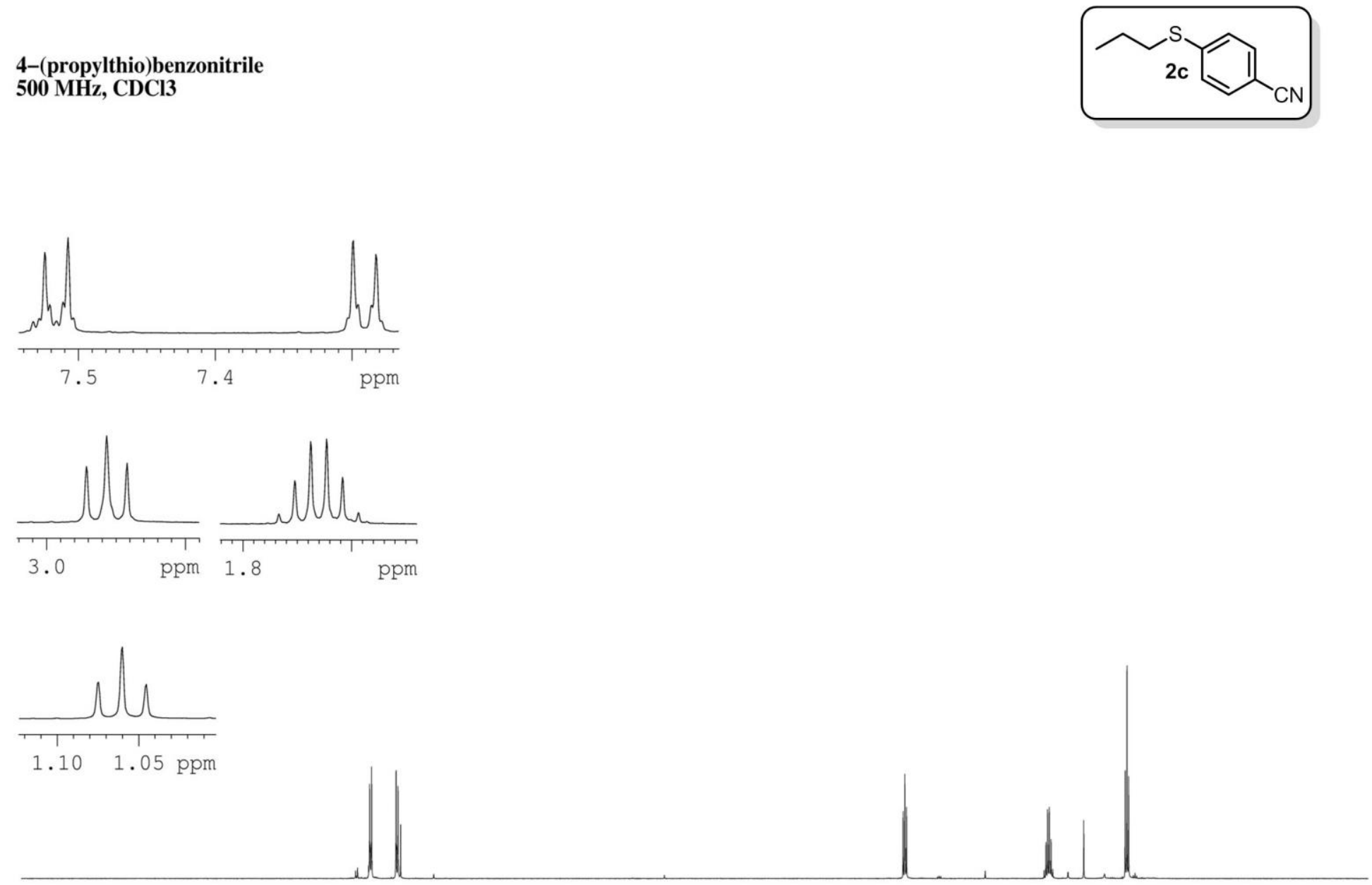

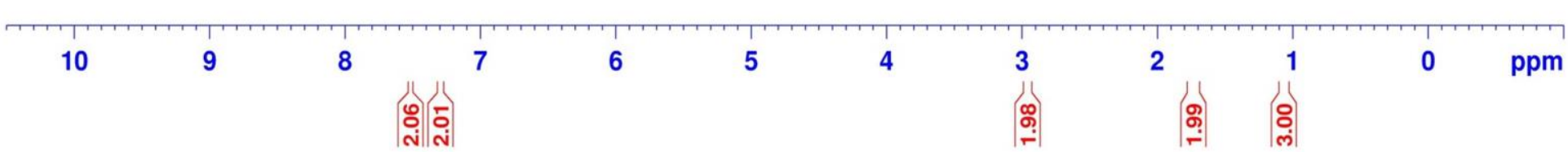


1-(4-(propylthio)phenyl)ethanone

$500 \mathrm{MHz}, \mathrm{CDCl} 3$
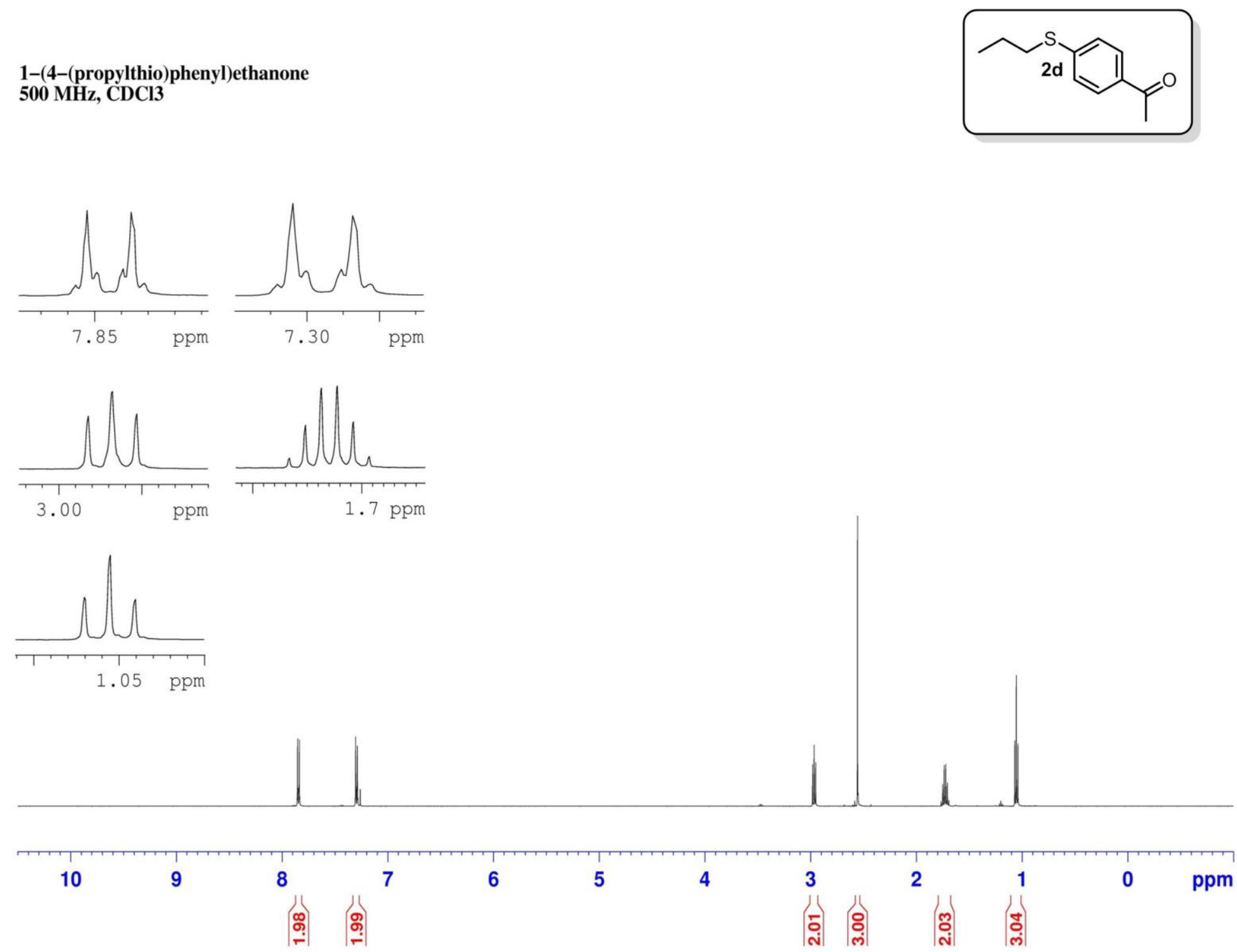
methyl 3-(propylthio)benzoate 500 MHz, CDCl3
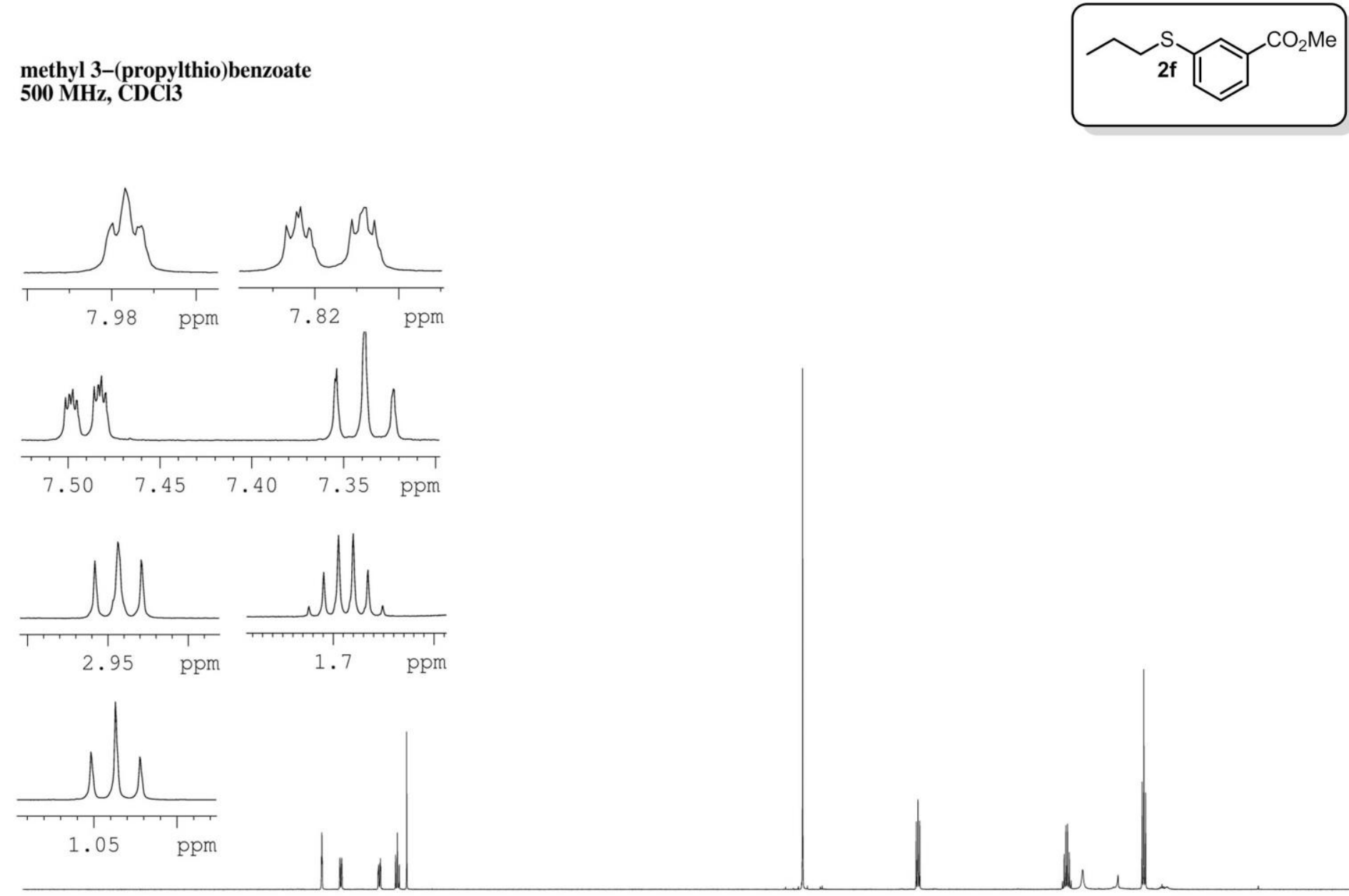

\begin{tabular}{|c|c|c|c|c|c|c|c|c|c|c|}
\hline 10 & 9 & 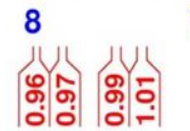 & 7 & 6 & 5 & 柋 & 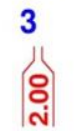 & 2 |) & $\begin{array}{l}1 \\
8 \\
0 \\
0\end{array}$ & 0 \\
\hline
\end{tabular}



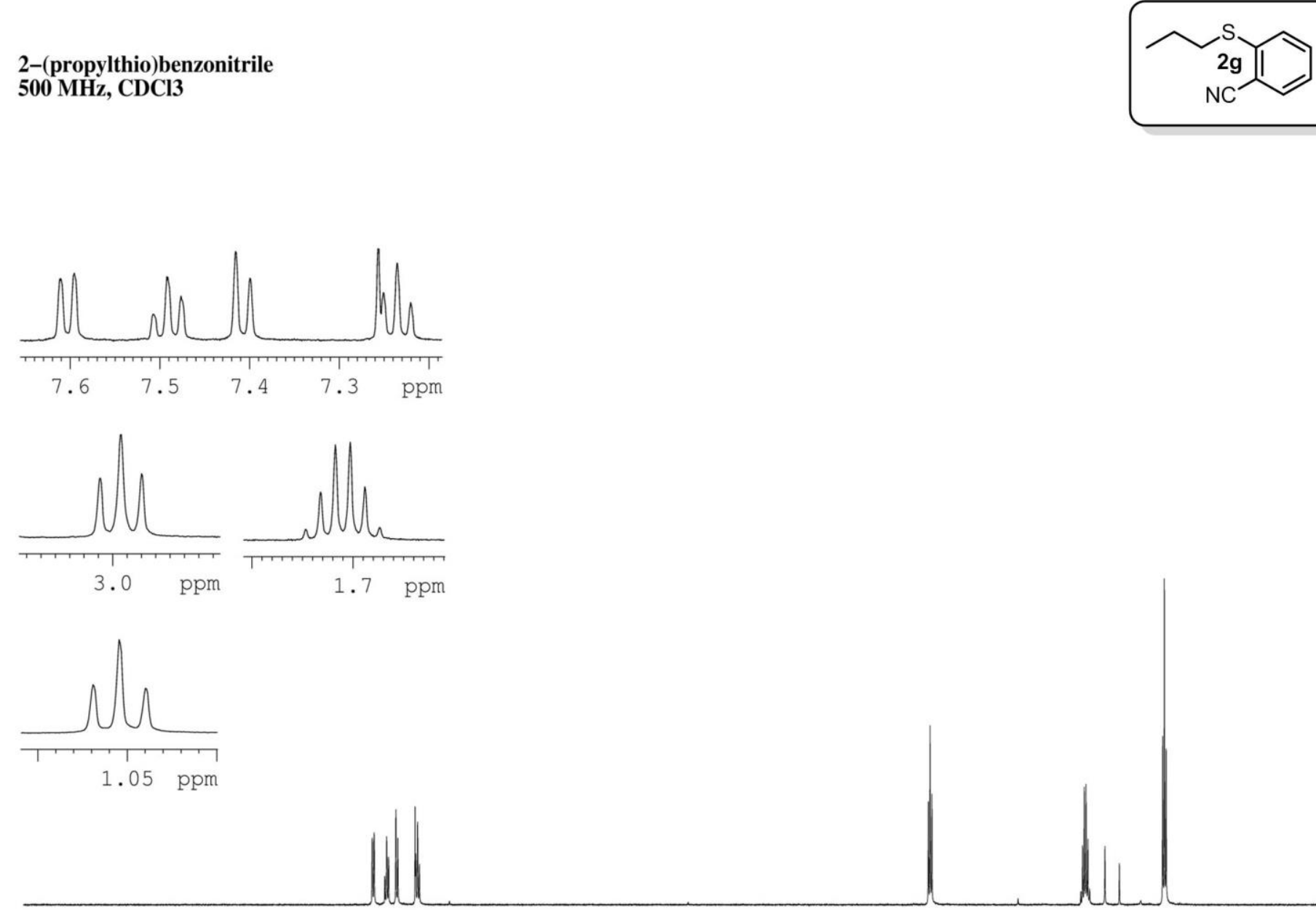

\begin{tabular}{|c|c|c|c|c|c|c|c|c|c|c|}
\hline 10 & 9 & 8 & 7 & 6 & 5 & 4 & $\begin{array}{c}3 \\
\left|\begin{array}{c}0 \\
\text { ஸे }\end{array}\right|\end{array}$ & 2 & $\begin{array}{l}1 \\
\varnothing \\
\dot{\mu}\end{array}$ & 0 \\
\hline
\end{tabular}


4-(propylthio)benzoic acid $500 \mathrm{MHz}, \mathrm{CDCl} 3$
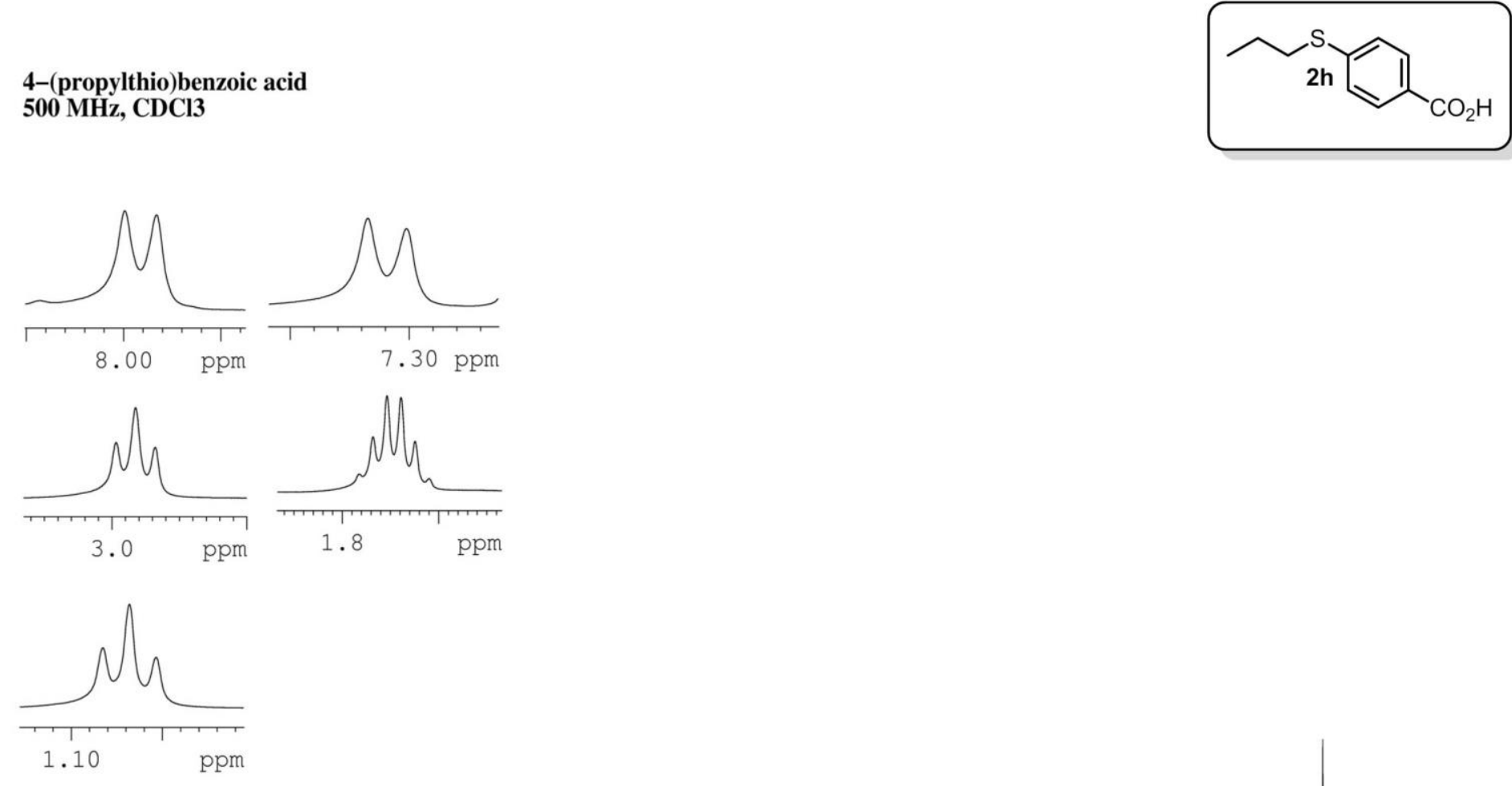

$$
\text { ppm }
$$

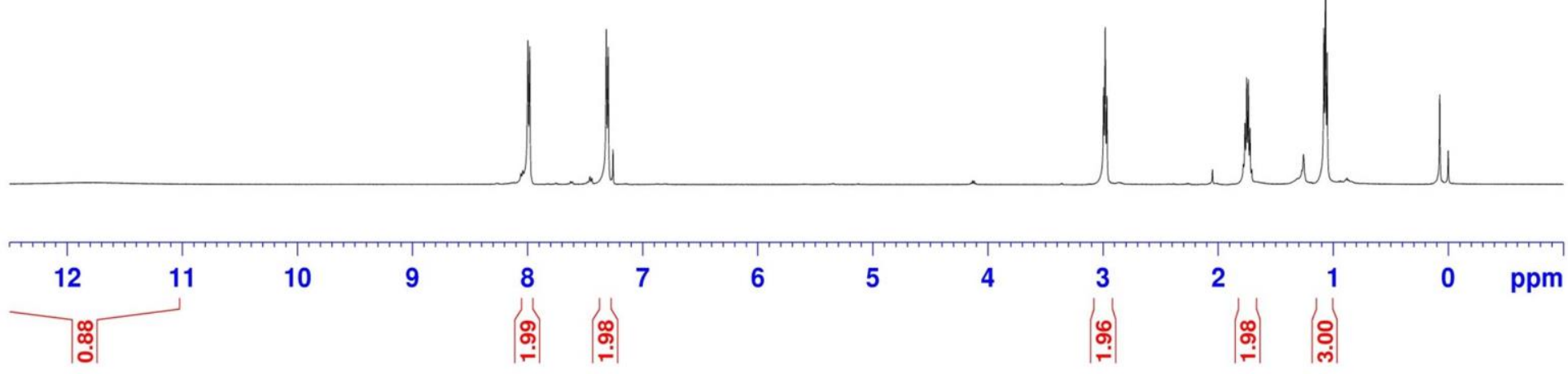


1-(5-(propylthio)thiophen-2-yl)ethanone $500 \mathrm{MHz}$, CDCl3
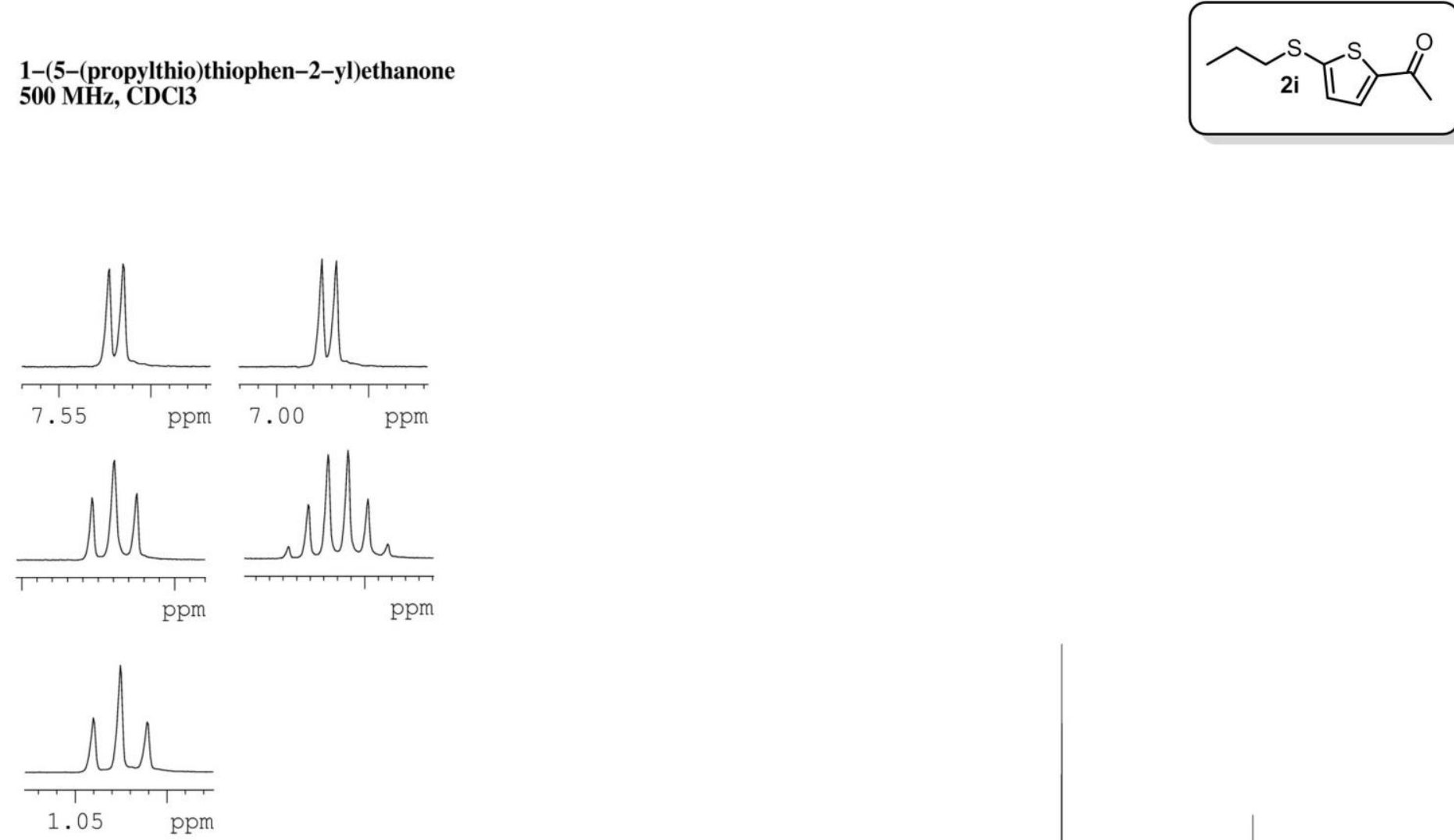

$$
\text { n }
$$

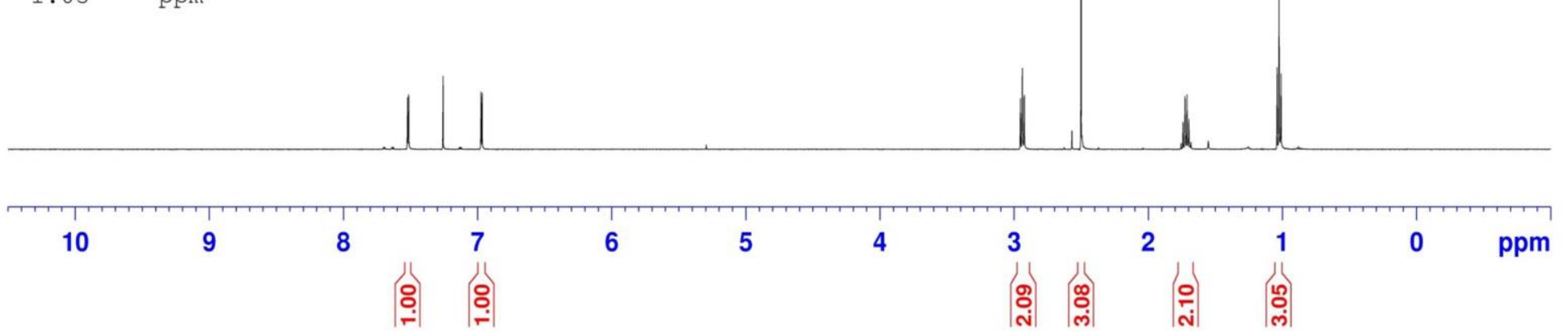


2-fluoro-4-(propylthio)pyridine

$500 \mathrm{MHz}, \mathrm{CDCl} 3$
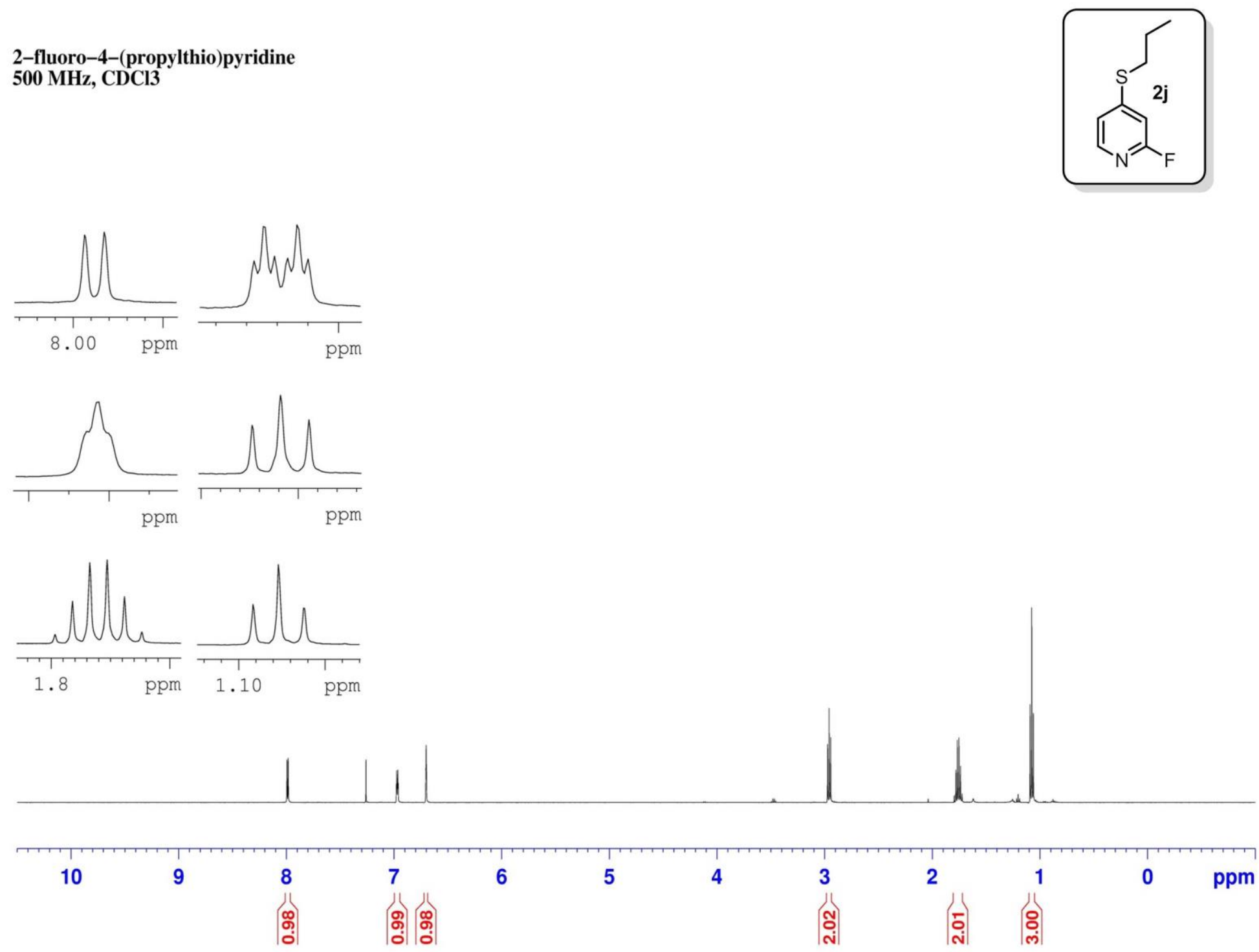
3-chloro-5-(propylthio)pyridine

$500 \mathrm{MHz}, \mathrm{CDCl} 3$
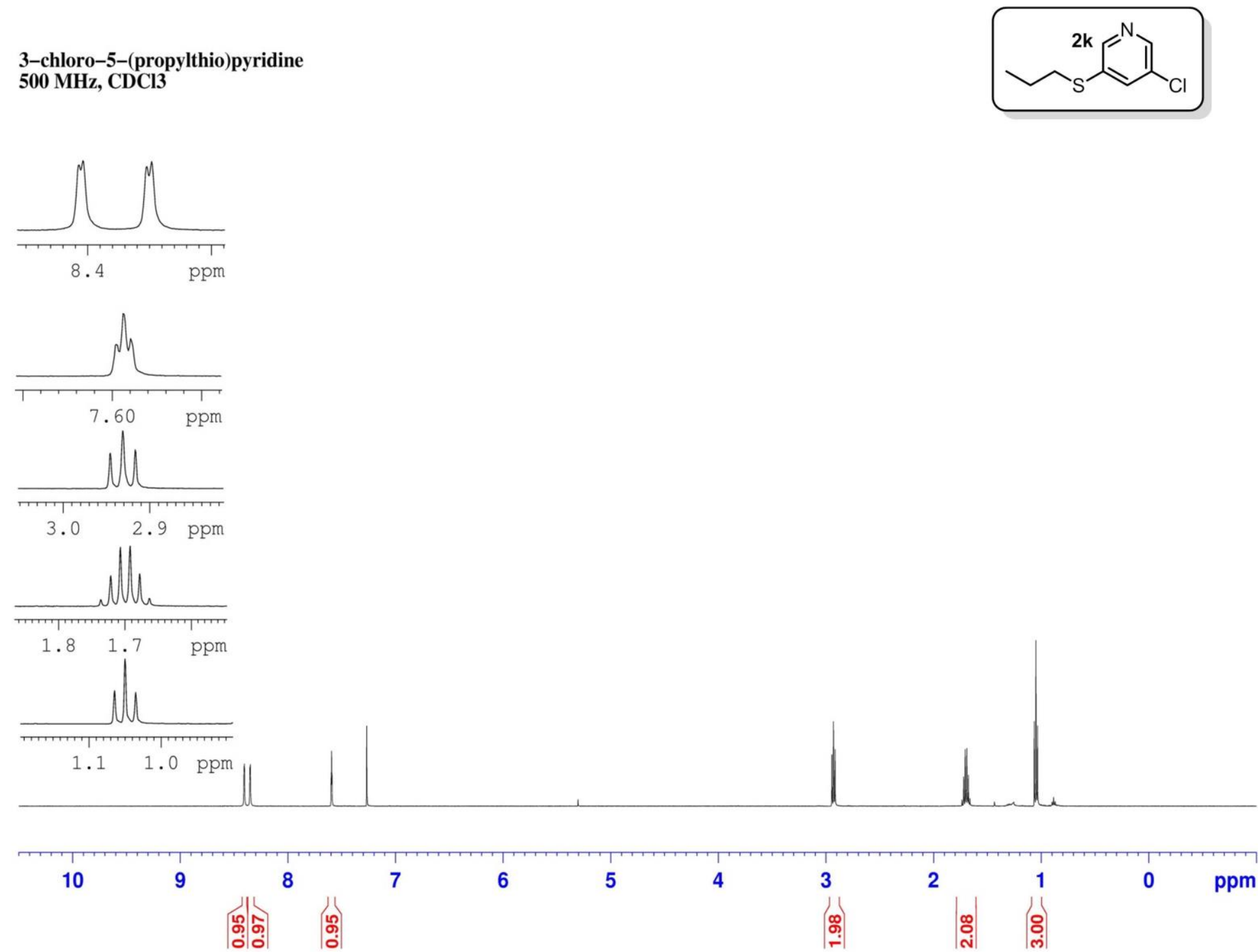
3-methoxy-5-(propylthio)pyridine $500 \mathrm{MHz}, \mathrm{CDCl} 3$
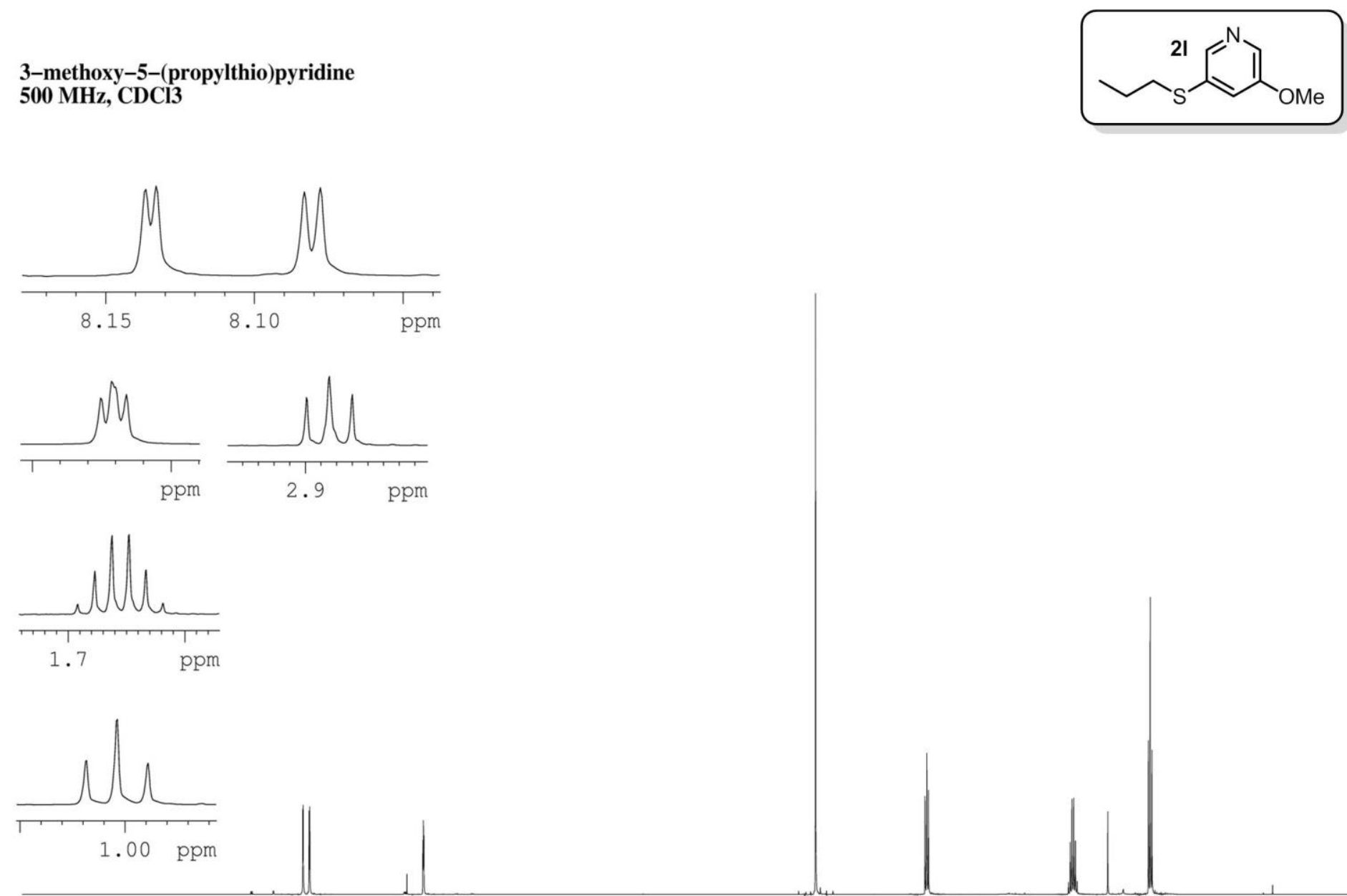

\begin{tabular}{|c|c|c|c|c|c|c|c|c|c|c|}
\hline 10 & 9 & 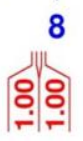 & 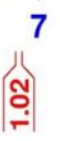 & 6 & 5 & & 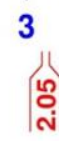 & 2 & $\begin{array}{c}1 \\
\vdots \\
0 \\
\dot{m}\end{array} \mid$ & 0 \\
\hline
\end{tabular}


5-methyl-2-(propylthio)pyridine $500 \mathrm{MHz}$, CDCl3
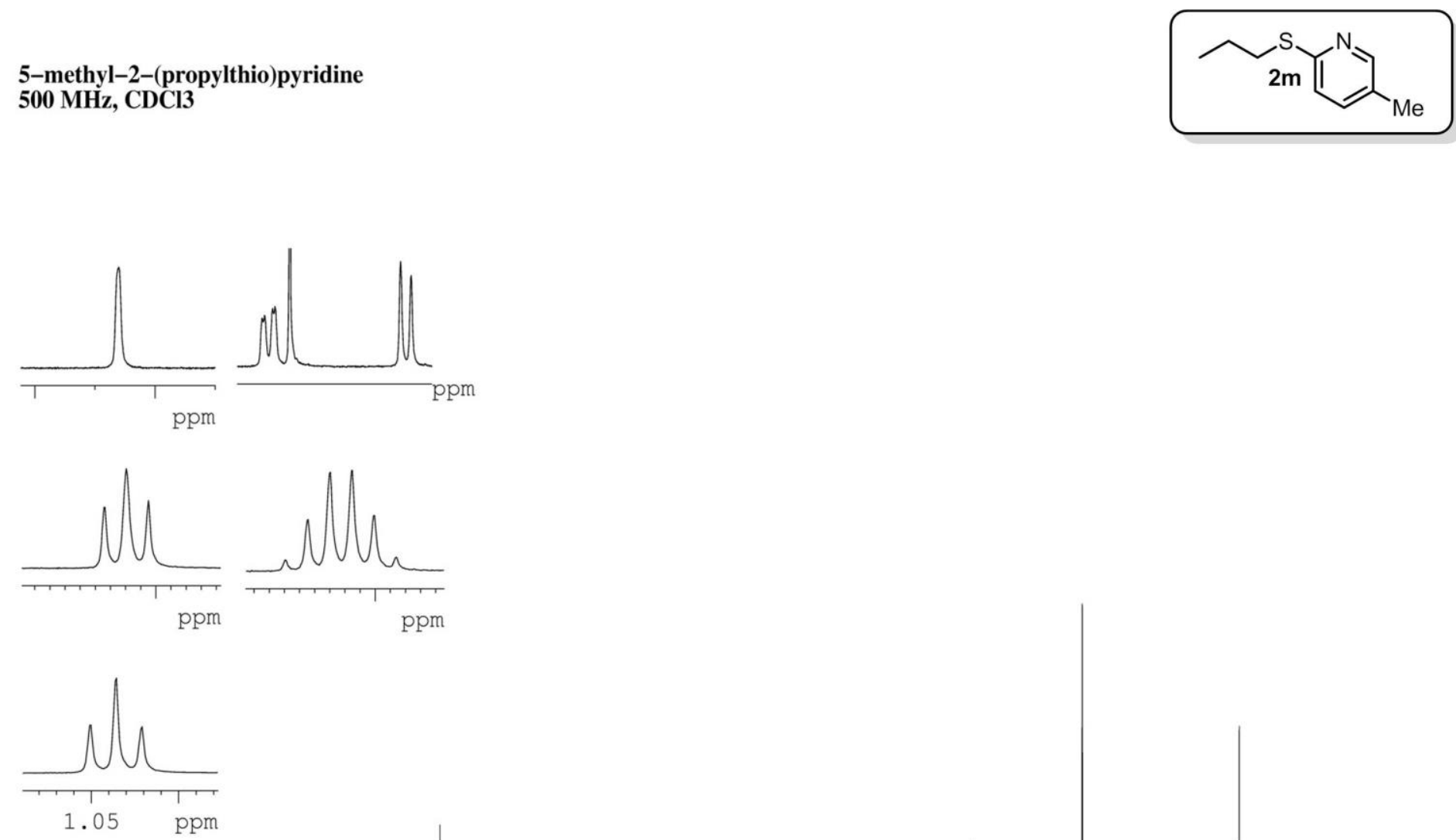

$$
\text { ppm }
$$
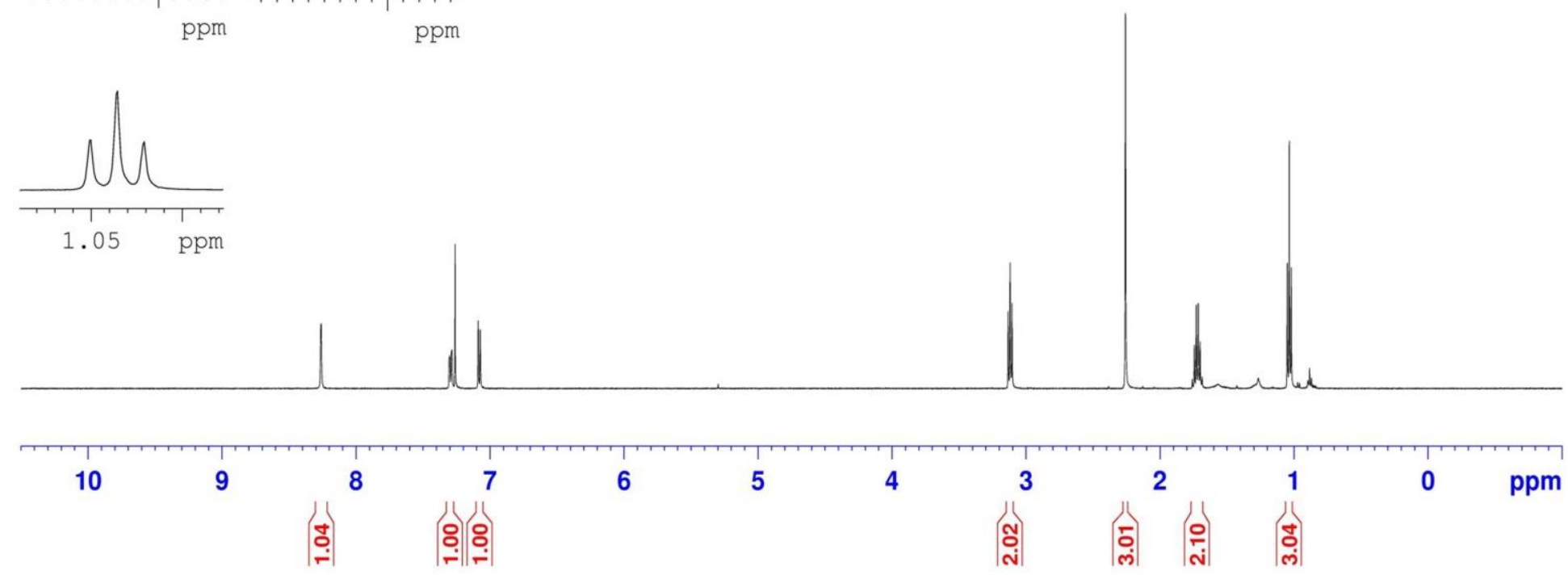
4-(propylthio)quinoline

$500 \mathrm{MHz}, \mathrm{CDCl} 3$
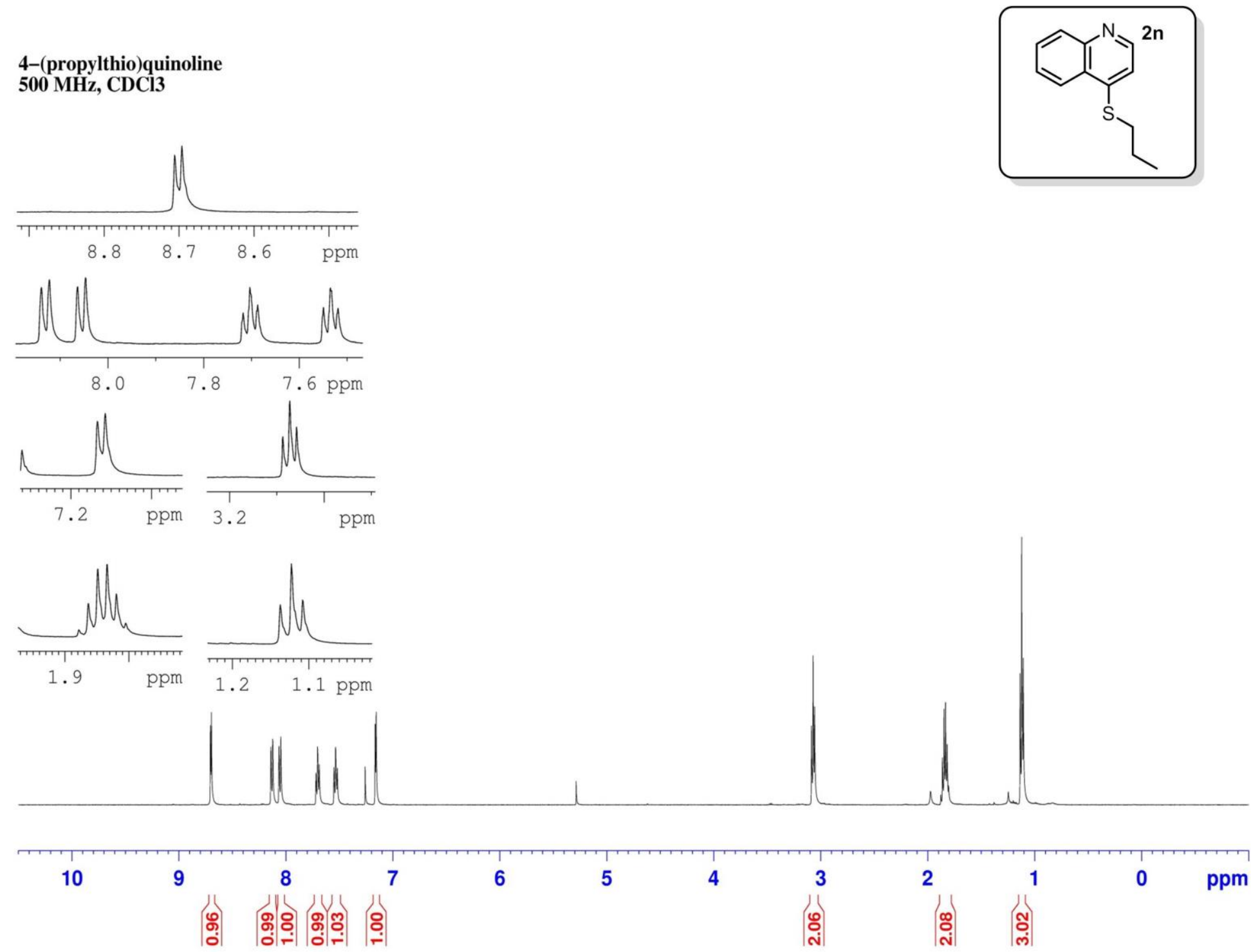
4-(propylthio)isoquinoline

$500 \mathrm{MHz}, \mathrm{CDCl} 3$
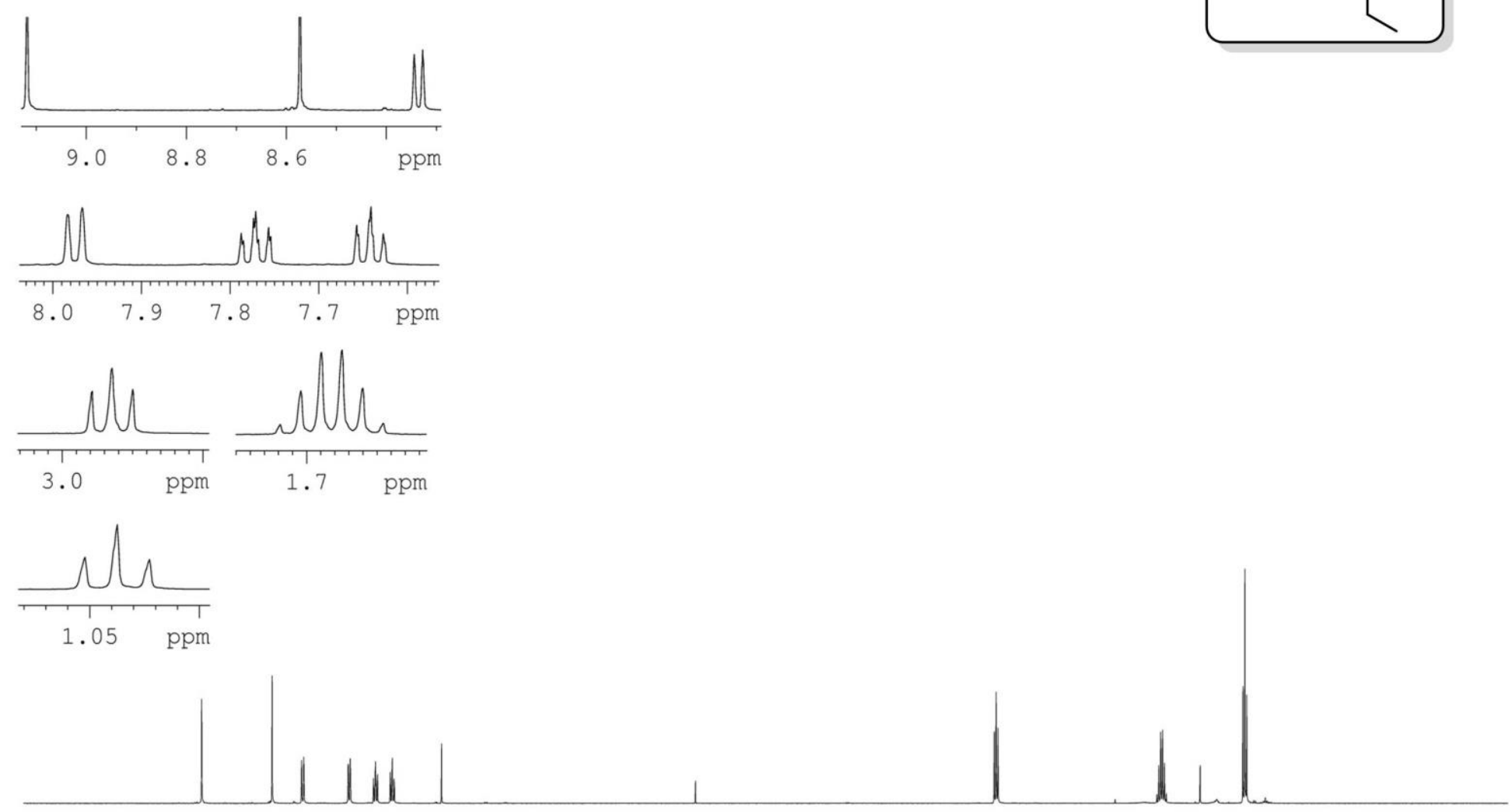

\begin{tabular}{|c|c|c|c|c|c|c|c|c|c|c|c|}
\hline 10 & $\begin{array}{l}9 \\
0 \\
0 \\
0\end{array}$ & 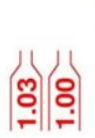 & 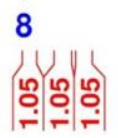 & 7 & 6 & 5 & 4 & $\begin{array}{l}3 \\
\left|\begin{array}{l}\infty \\
0 \\
\text { î. }\end{array}\right|\end{array}$ & 2 & $\begin{array}{l}1 \\
0 \\
0 \\
0 \\
\text { m. }\end{array}$ & 0 \\
\hline
\end{tabular}


8-(propylthio)isoquinoline

$500 \mathrm{MHz}, \mathrm{CDCl} 3$
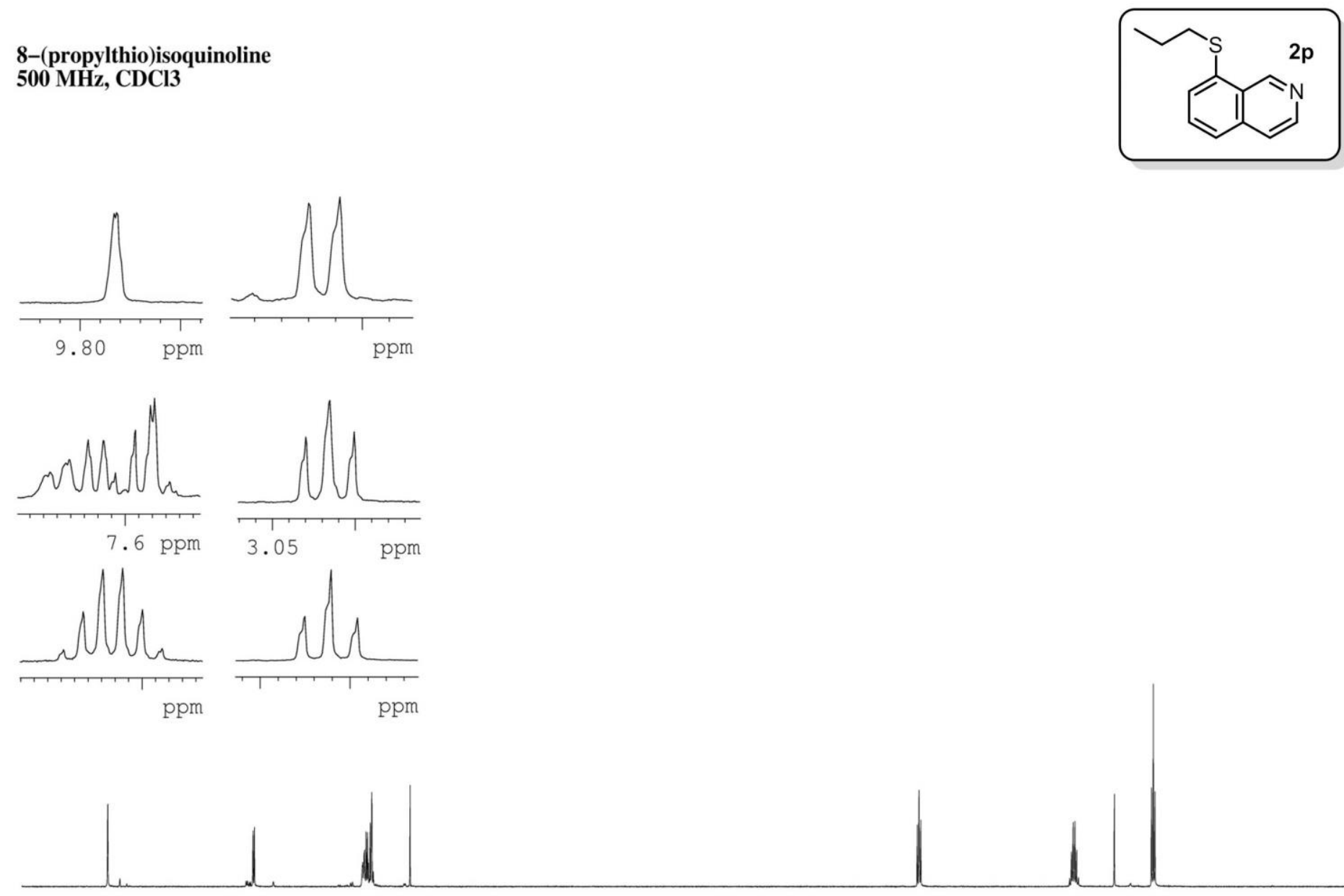

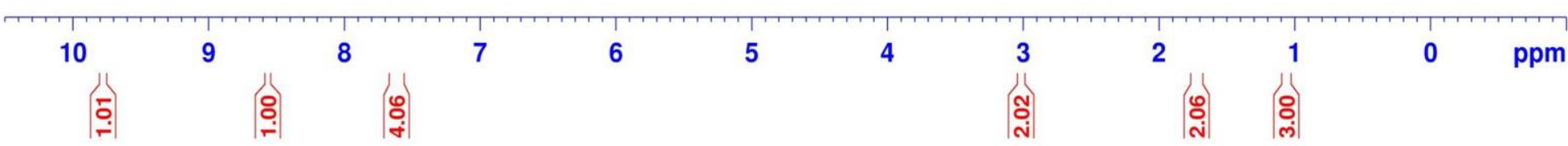


tert-butyl 5-(propylthio)-1H-indazole-1-carboxylate $500 \mathrm{MHz}, \mathrm{CDCl} 3$
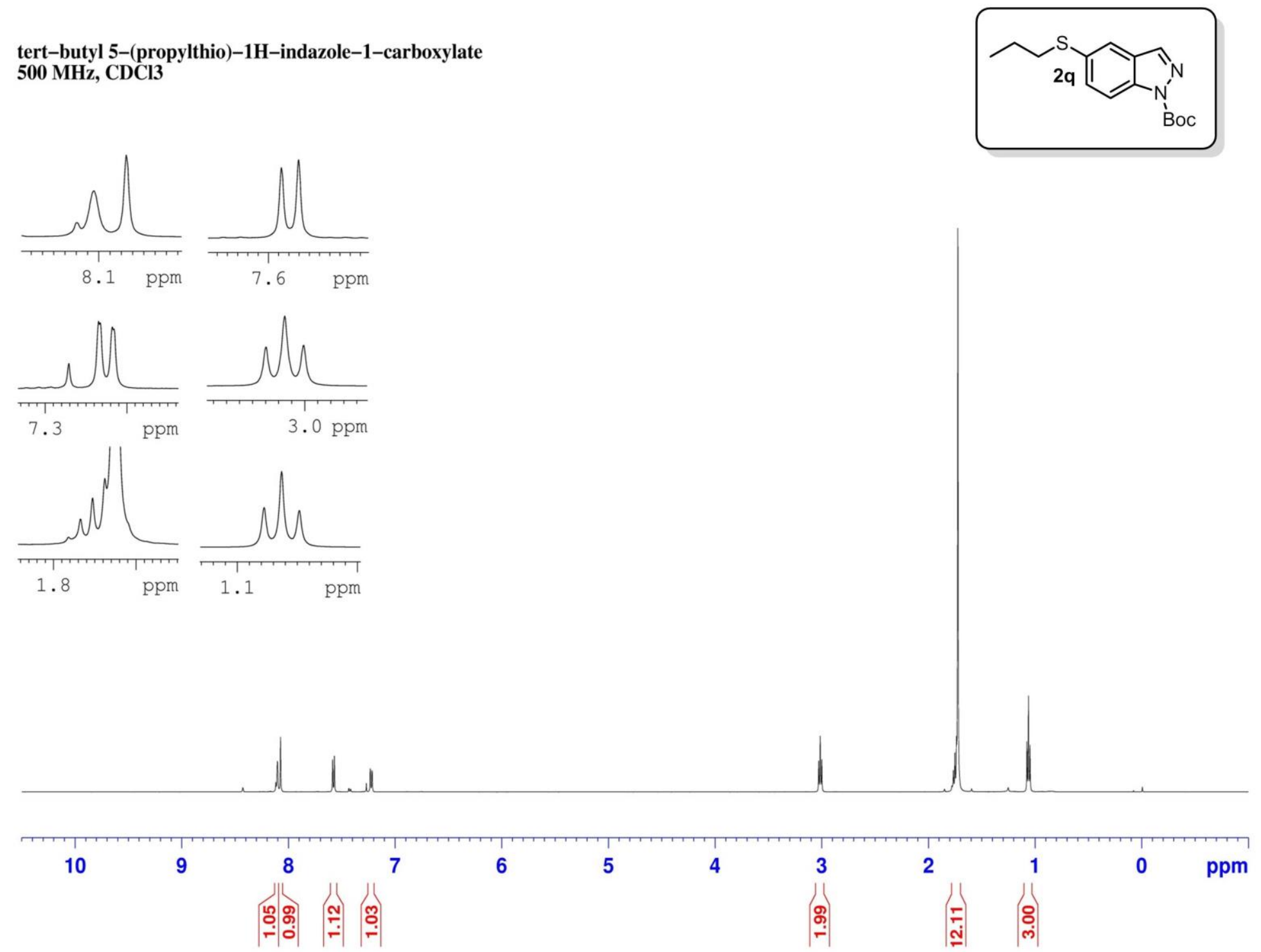
1,3,7-trimethyl-8-(propylthio)-1H-purine-2,6(3H,7H)-dione $500 \mathrm{MHz}, \mathrm{CDCl} 3$
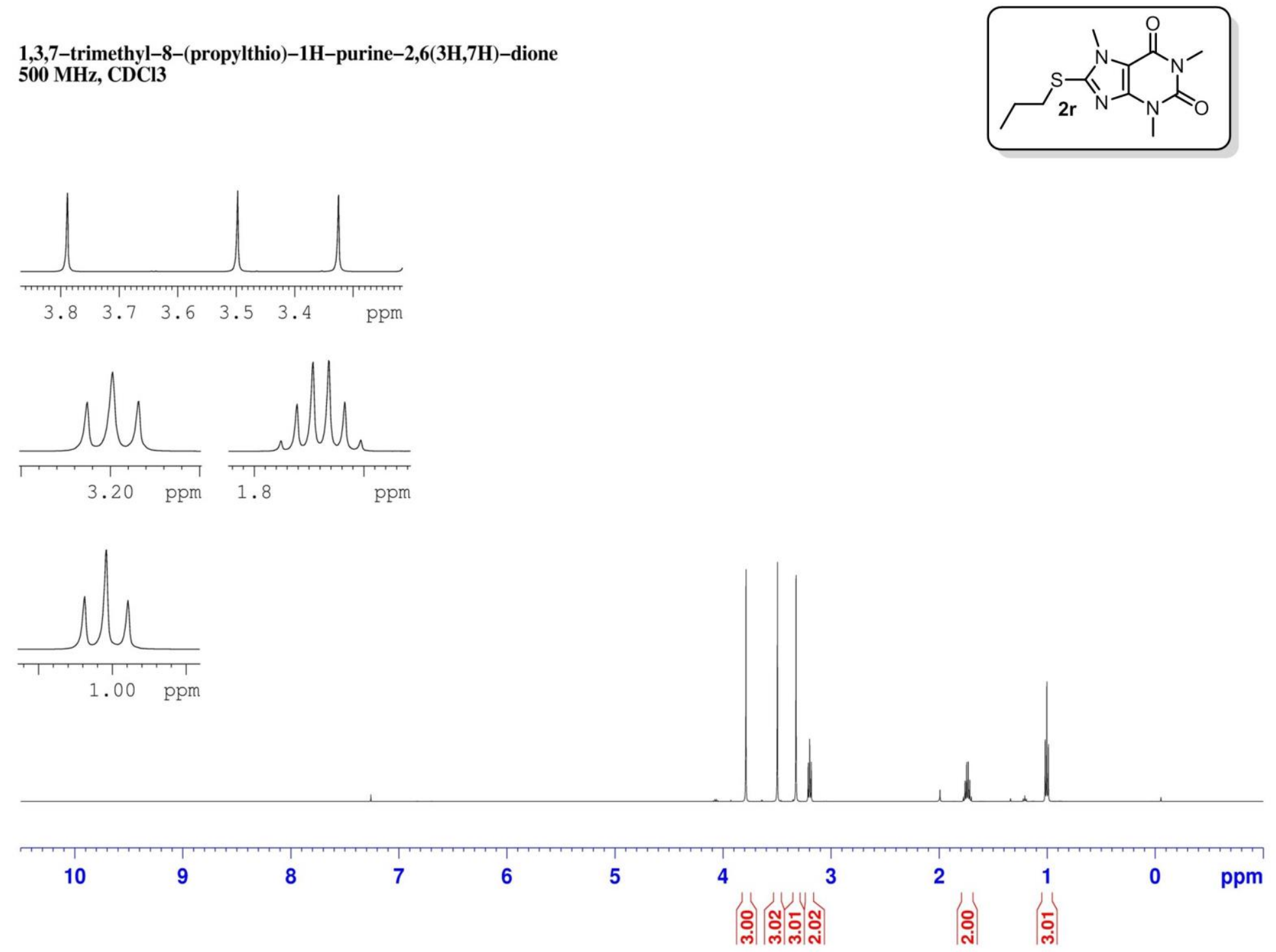
4-(butylthio)benzonitrile

$500 \mathrm{MHz}, \mathrm{CDCl} 3$
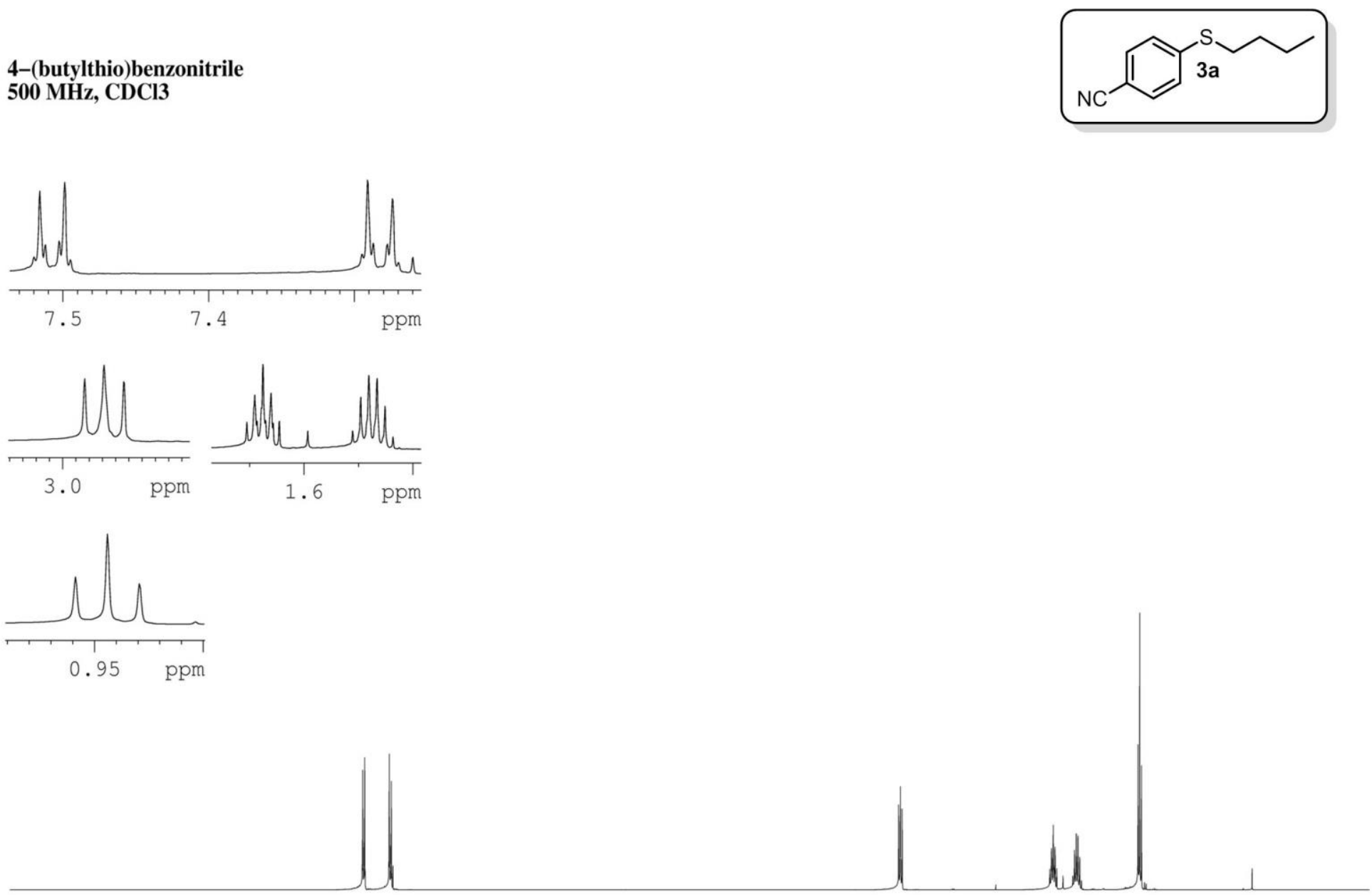

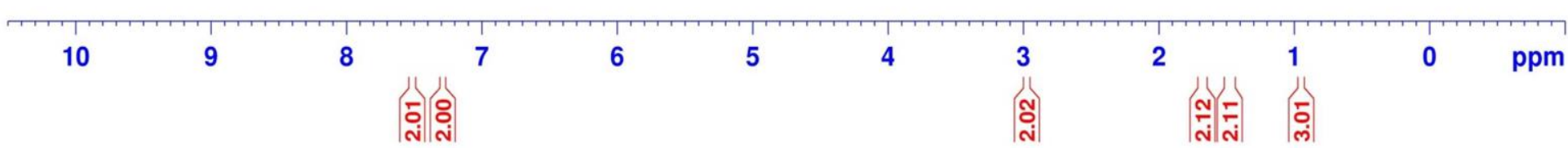



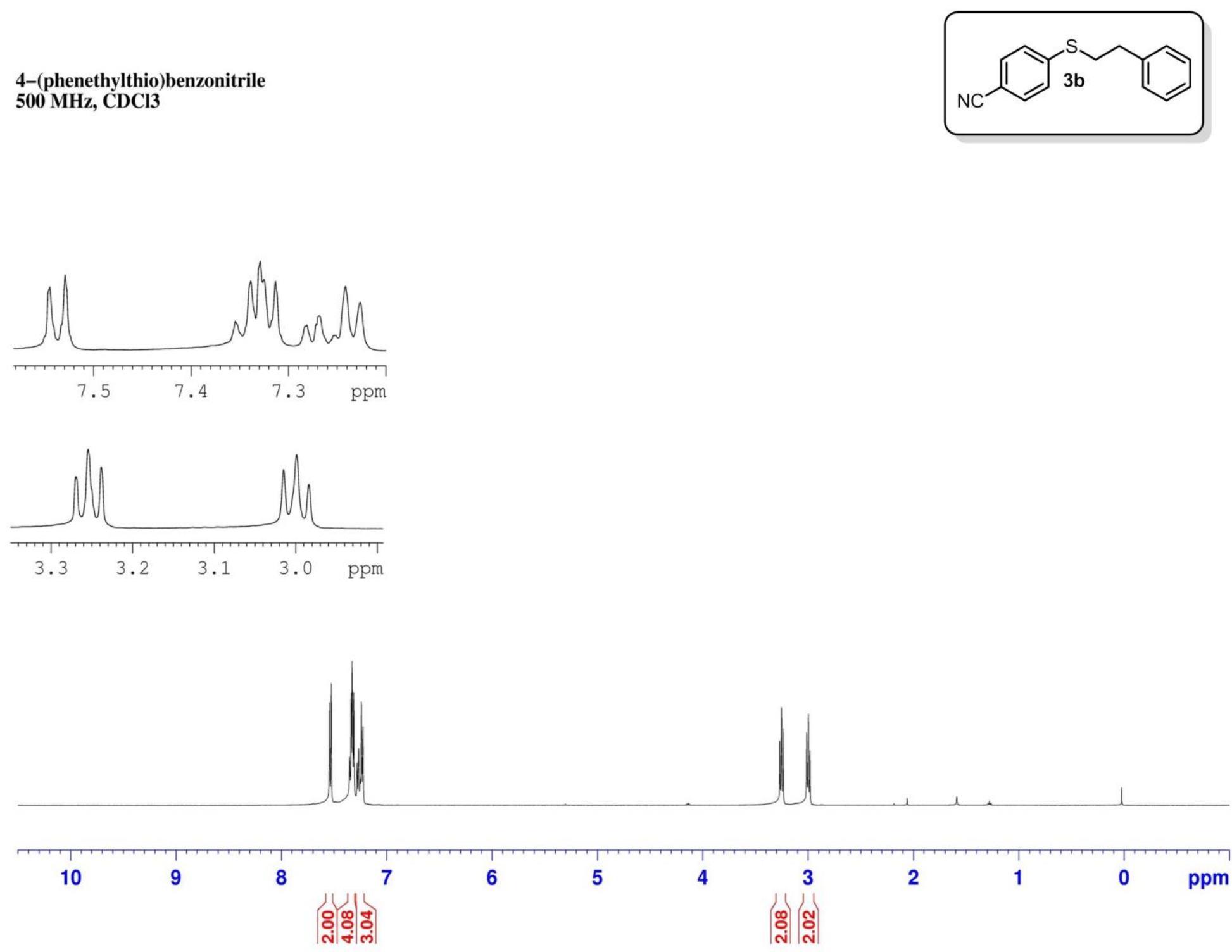
methyl 3-((4-cyanophenyl)thio)propanoate $500 \mathrm{MHz}, \mathrm{CDCl} 3$
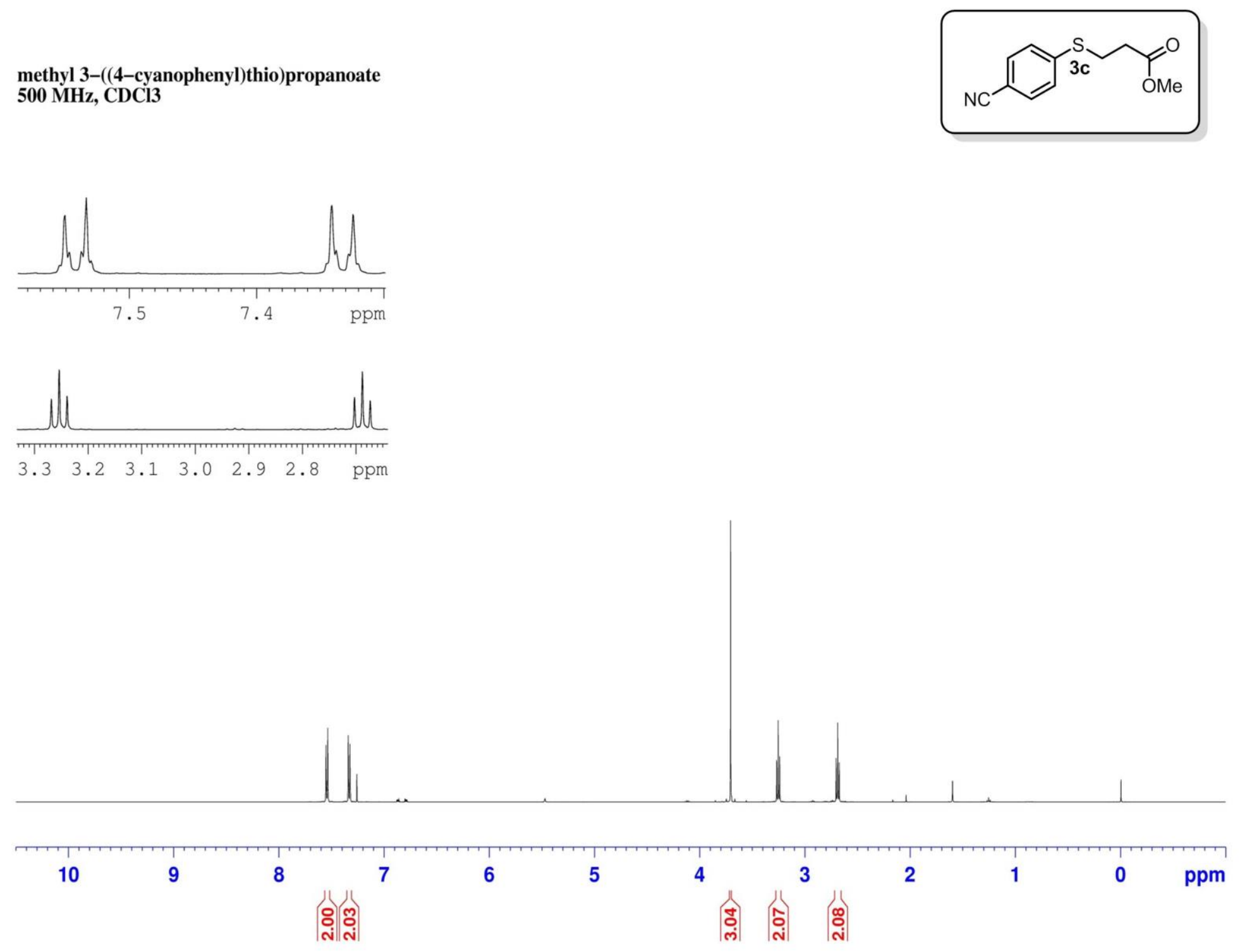
4-((2-hydroxyethyl)thio)benzonitrile $500 \mathrm{MHz}, \mathrm{CDCl} 3$
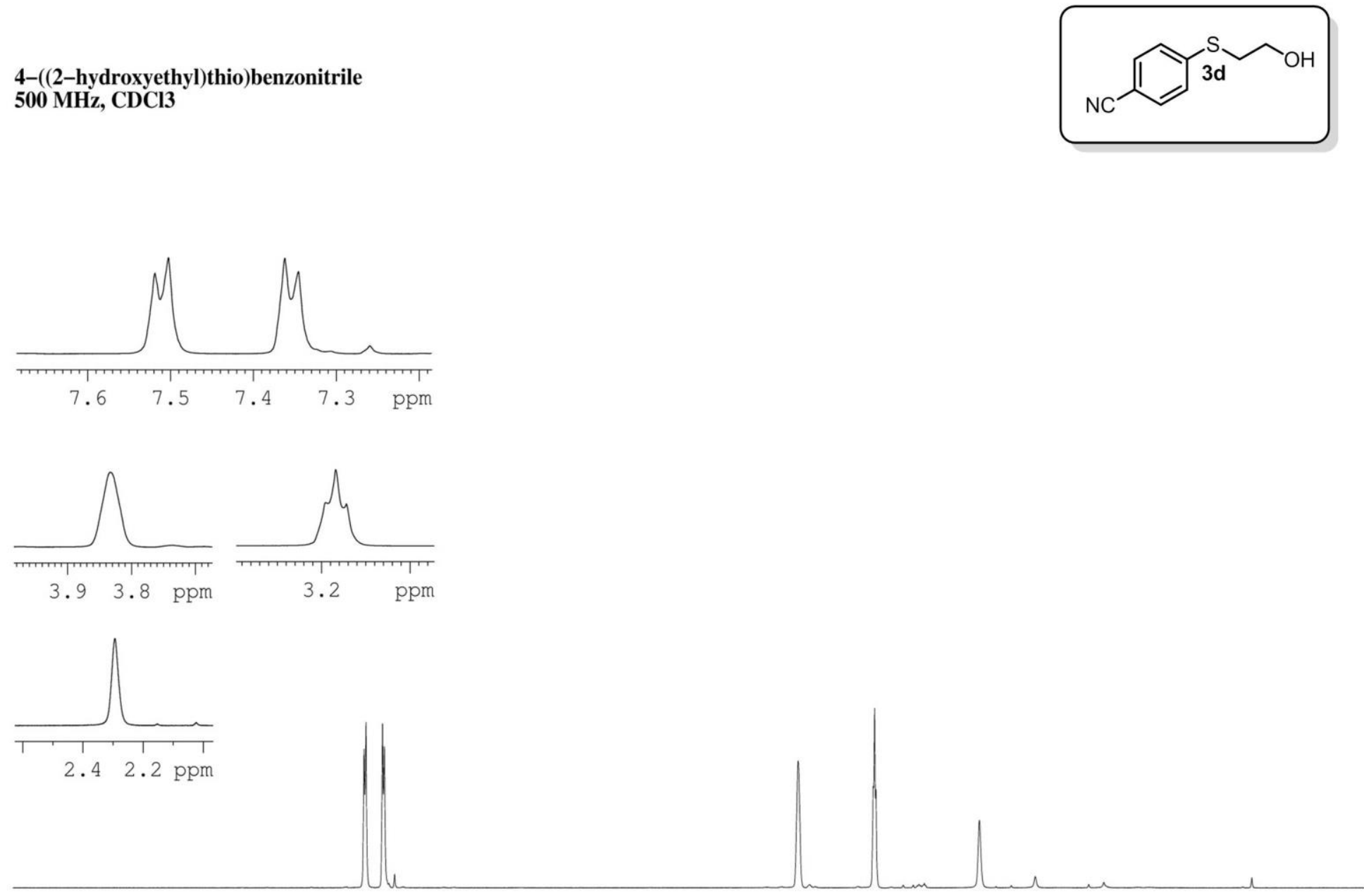

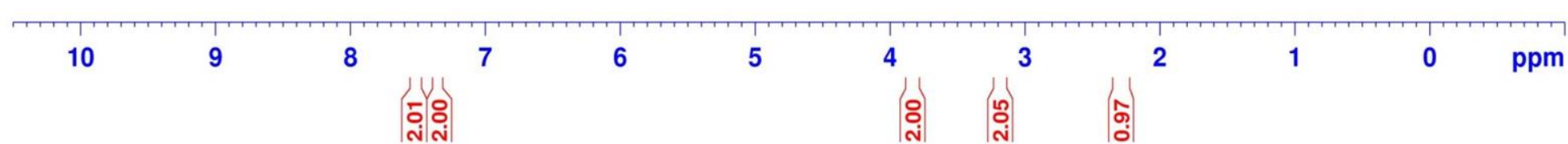


4-((2-aminoethyl)thio)benzonitrile $500 \mathrm{MHz}, \mathrm{CDCl} 3$
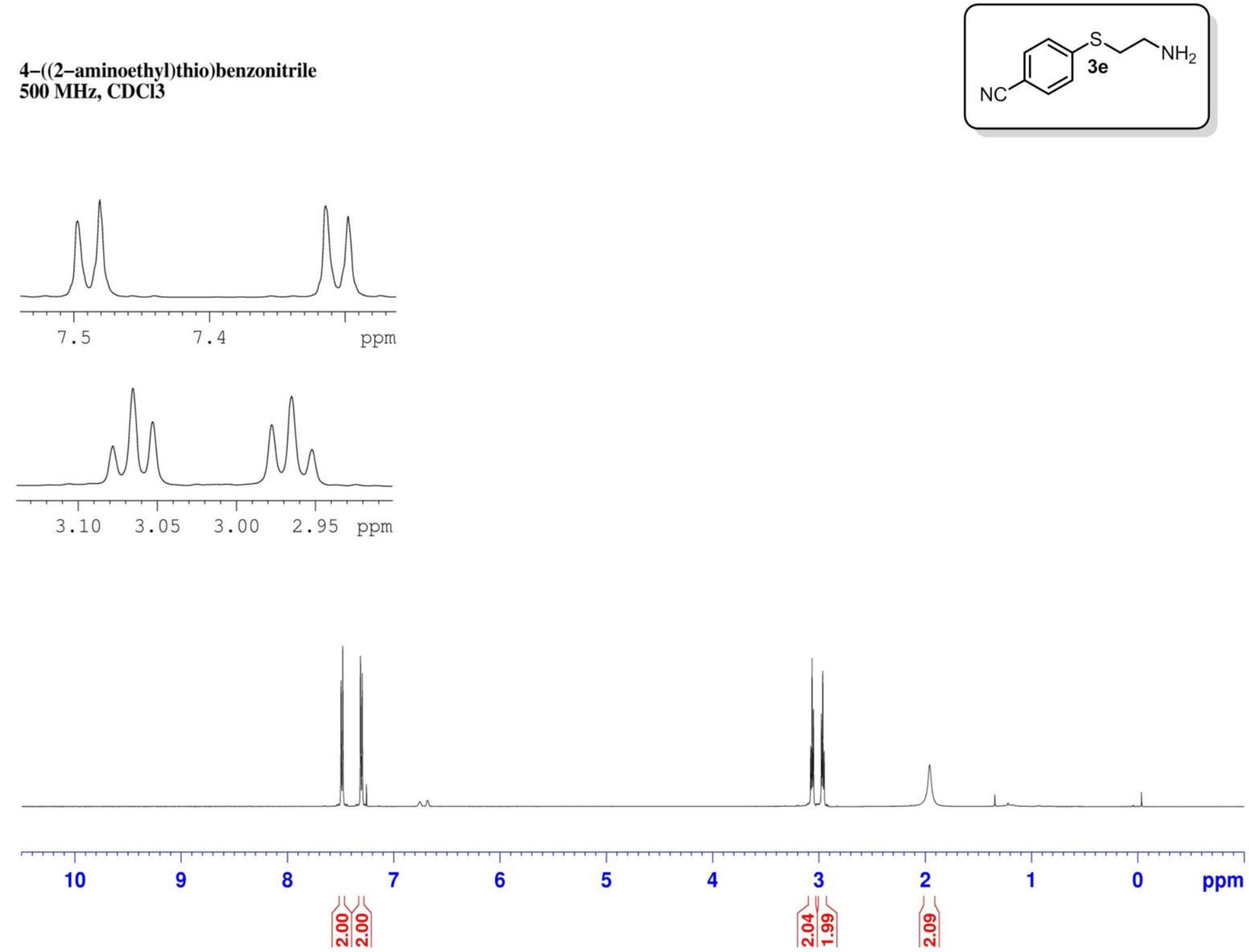
4-(cyclohexylthio)benzonitrile $500 \mathrm{MHz}, \mathrm{CDCl} 3$
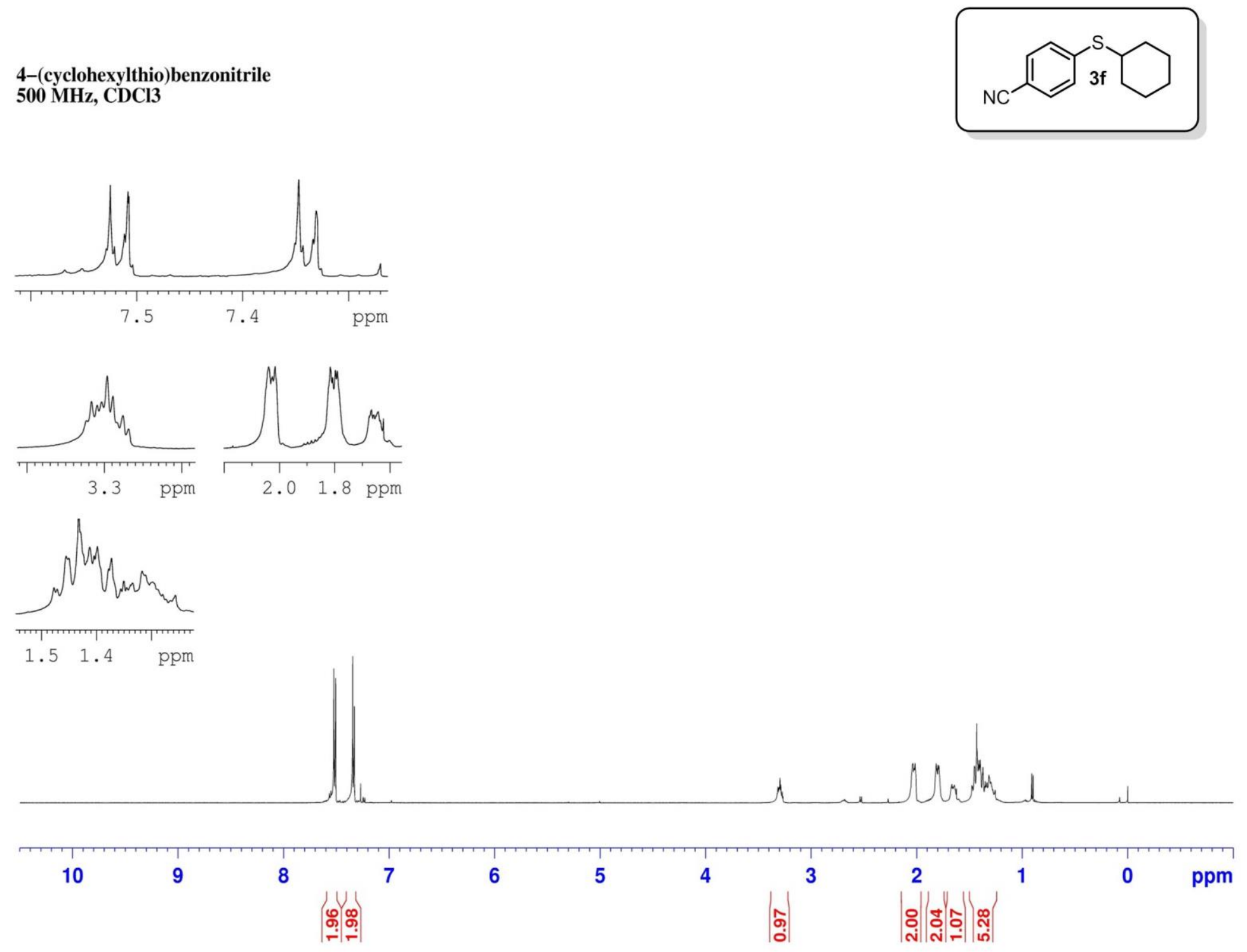
4-((1-hydroxyhexan-3-yl)thio)benzonitrile $500 \mathrm{MHz}, \mathrm{CDCl} 3$
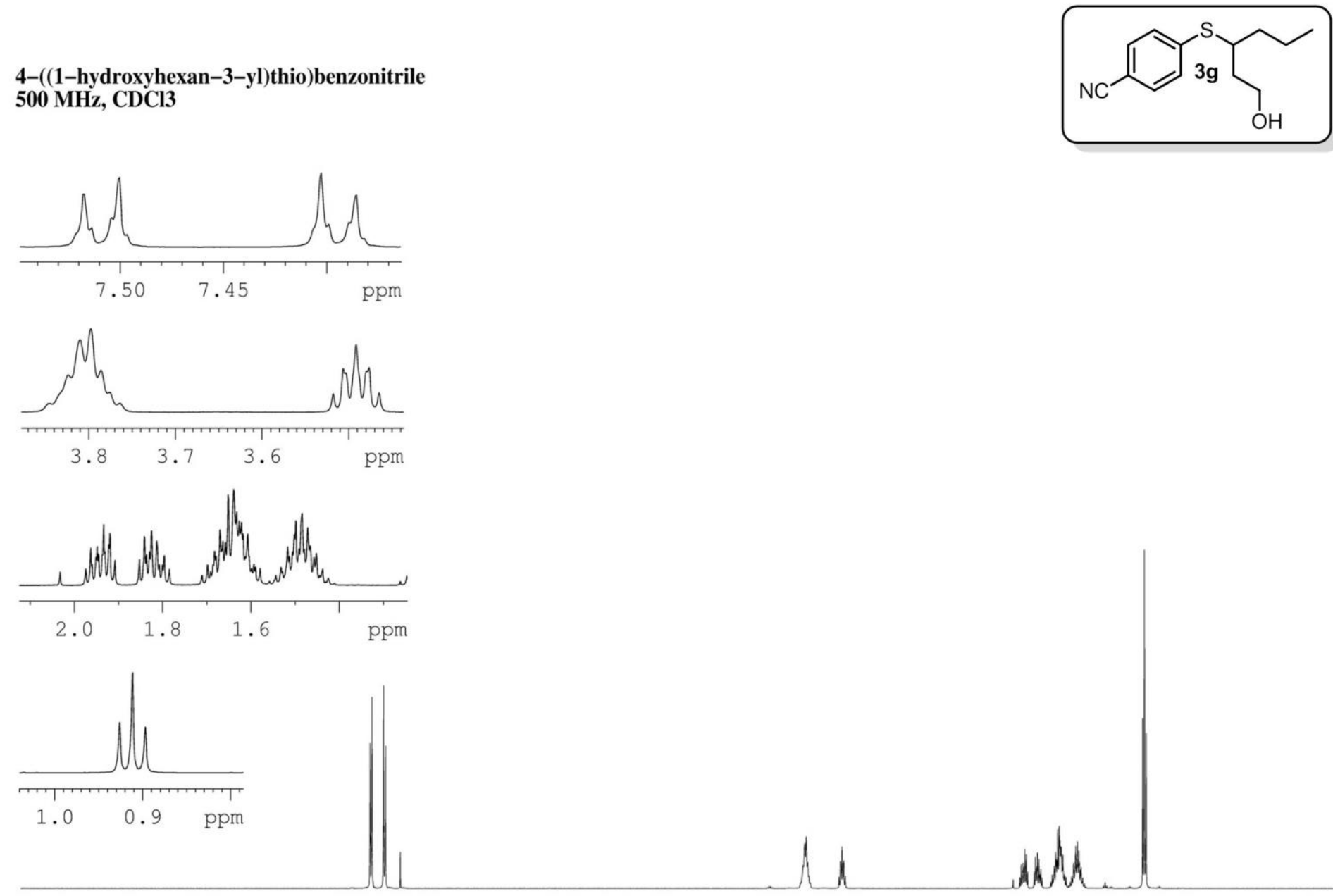

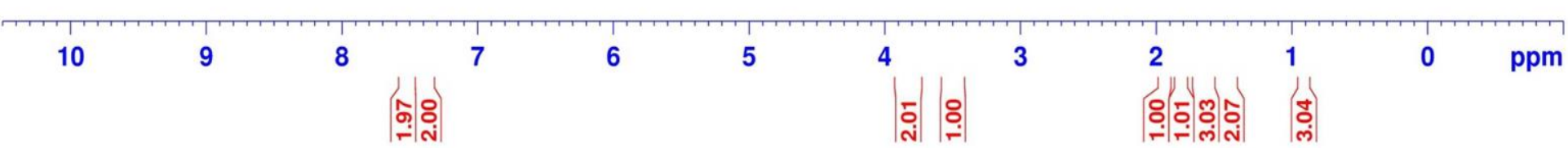


R)-methyl 2-((tert-butoxycarbonyl)amino)-3-((4-cyanophenyl)thio)propanoate $500 \mathrm{MHz}, \mathrm{CDCl} 3$
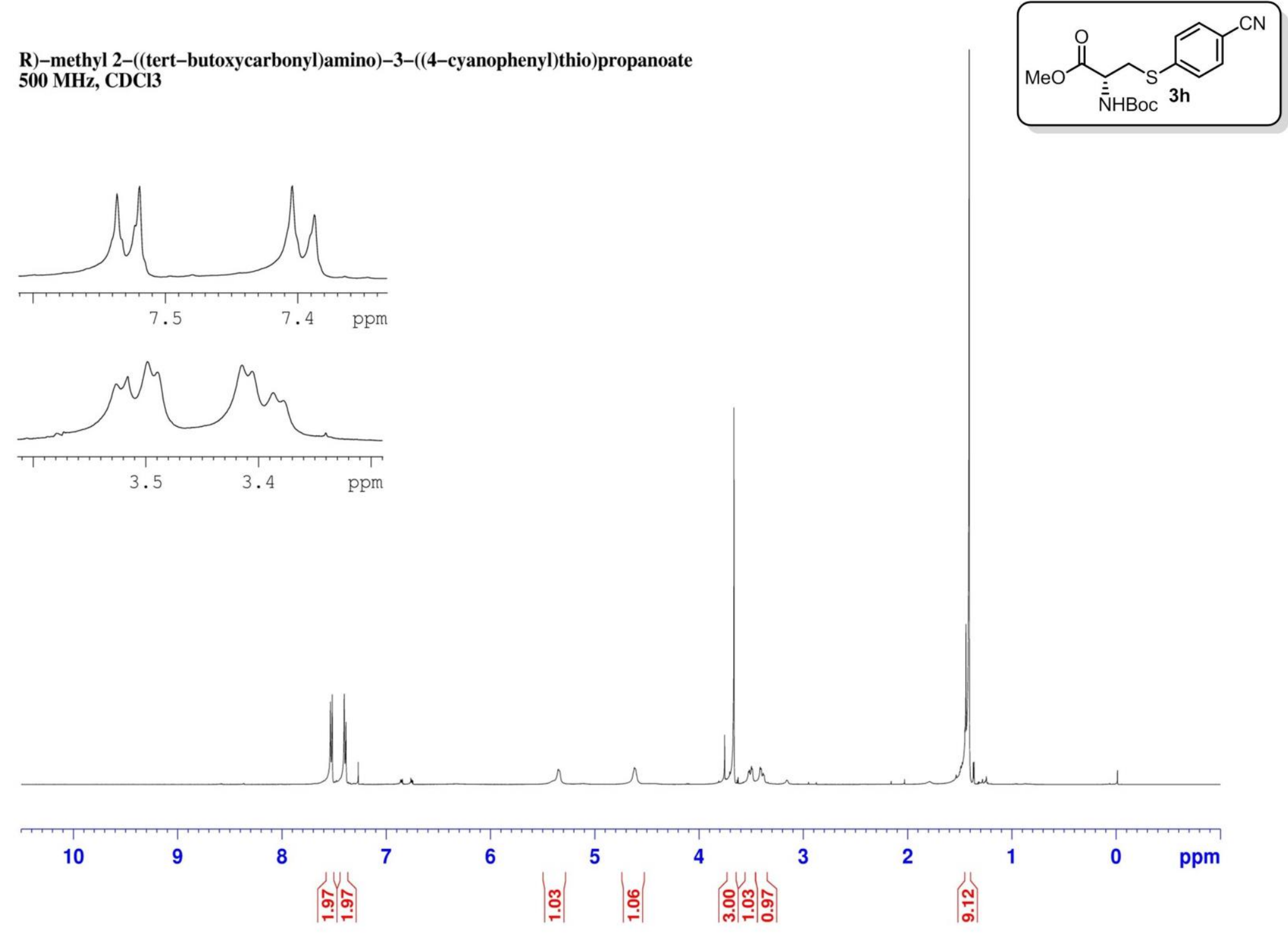
(4-(tert-butylthio)benzonitrile $500 \mathrm{MHz}, \mathrm{CDCl} 3$
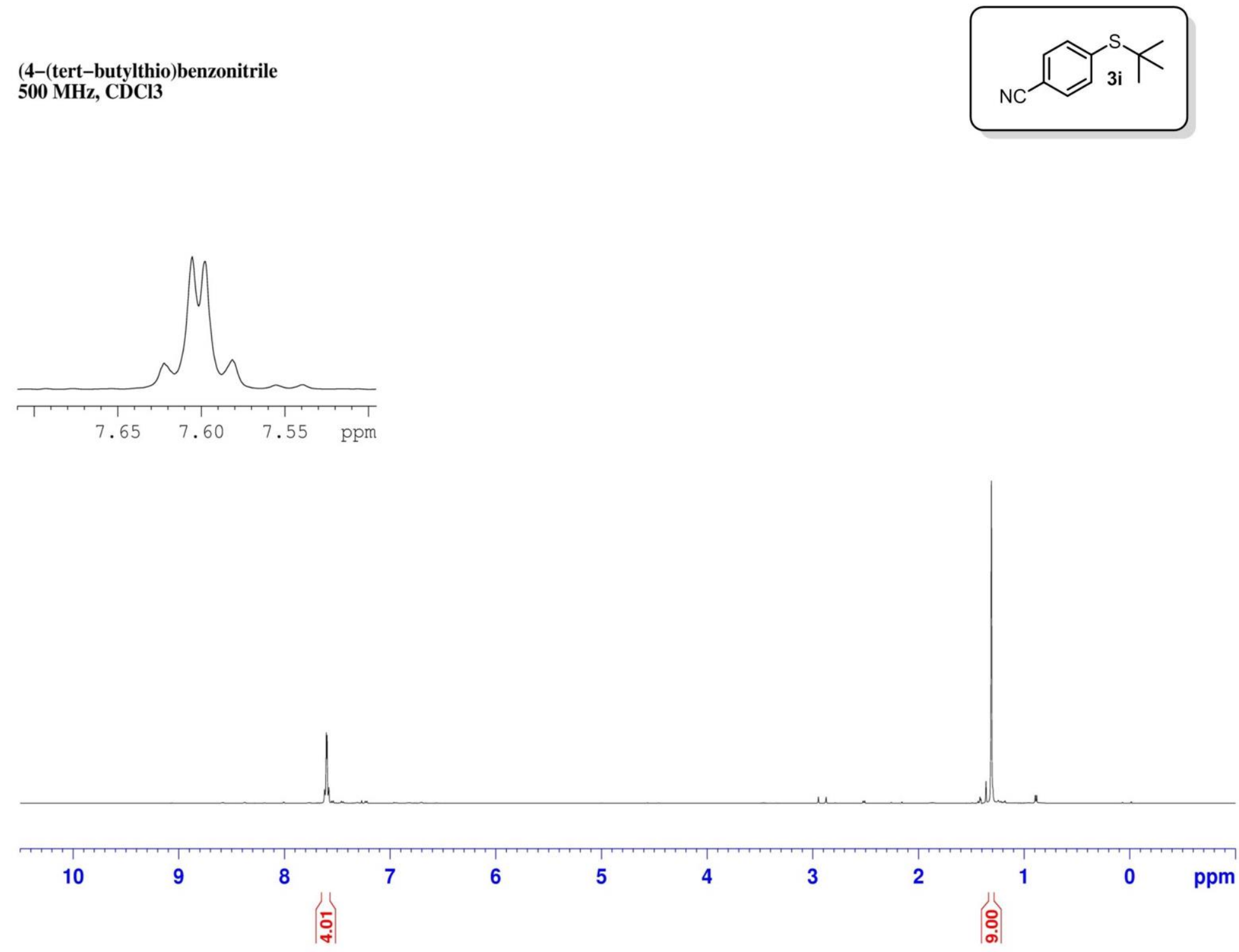
4-(adamantan-1-ylthio)benzonitrile $500 \mathrm{MHz}, \mathrm{CDCl} 3$
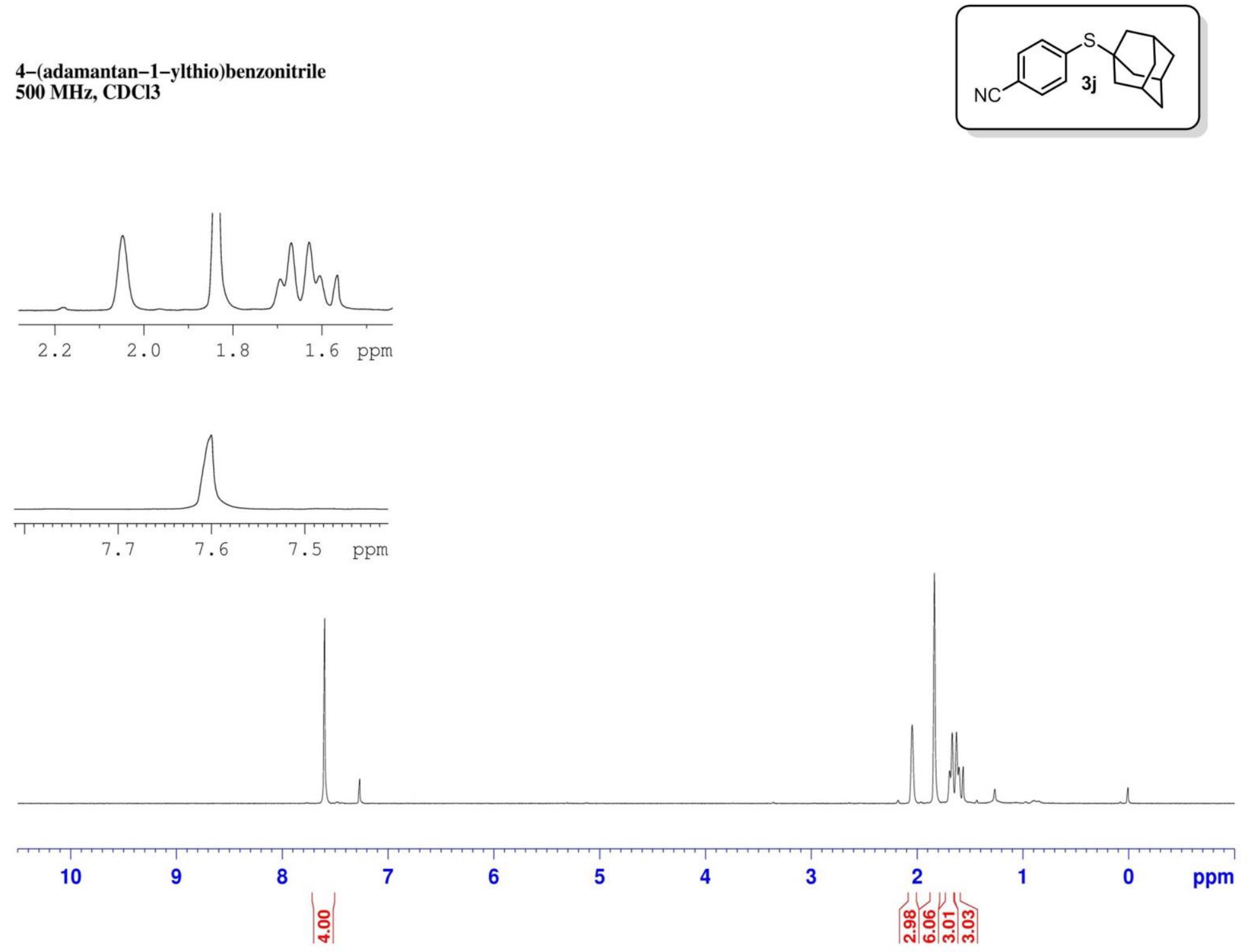
(1S,4S)- \& (1R,4R)-4-((2-(4-Methyl-2-oxocyclohexyl)propan-2-yl)thio)benzonitrile $500 \mathrm{MHz}, \mathrm{CDCl} 3$
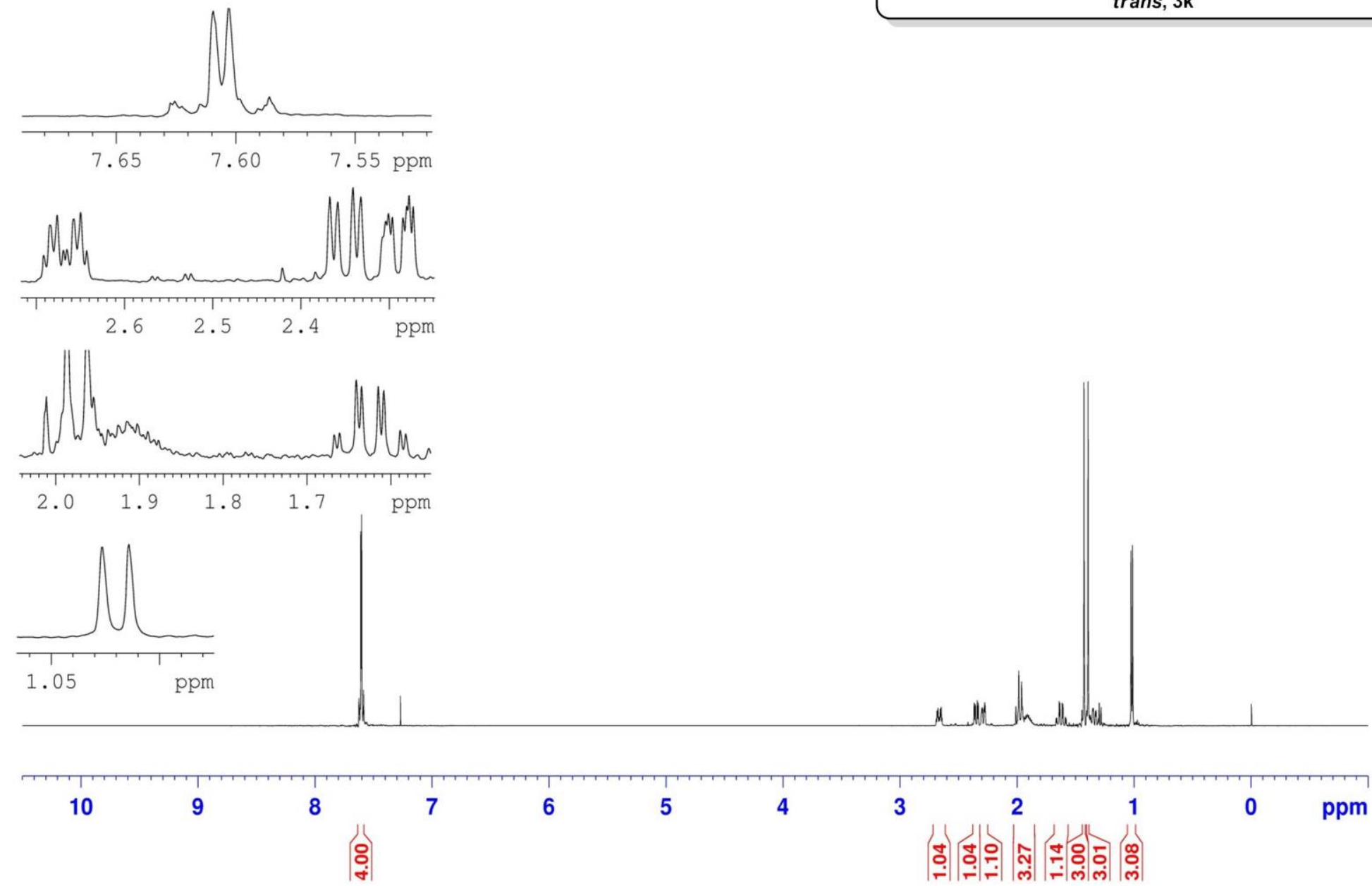
(1R,4S)- \& (1R,4S)-4-((2-(4-Methyl-2-oxocyclohexyl)propan-2-yl)thio)benzonitrile $500 \mathrm{MHz}, \mathrm{CDCl} 3$
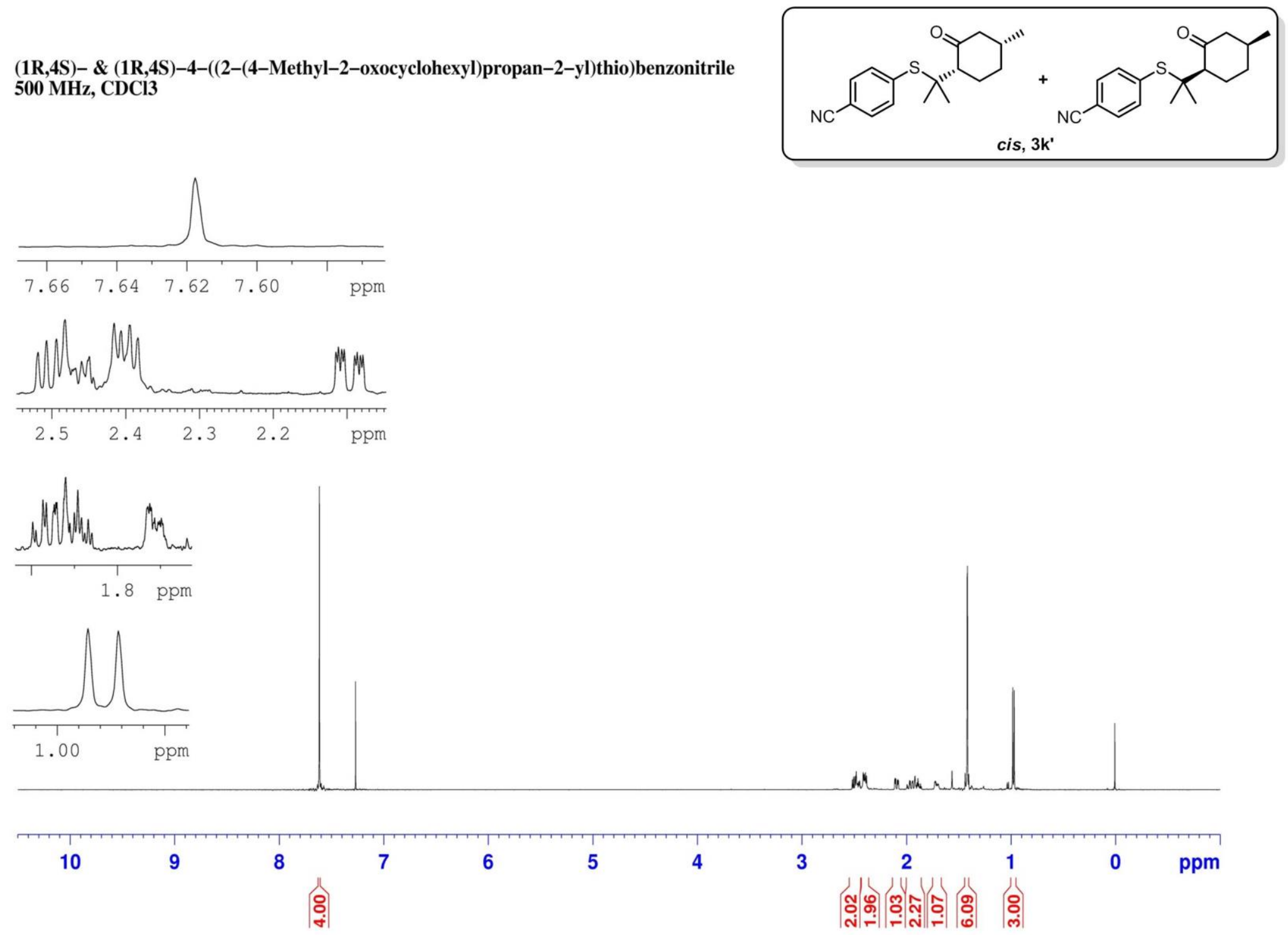
(R)-methyl 2-((tert-butoxycarbonyl)amino)-3-((2-fluoropyridin-4-yl)thio)propanoate $500 \mathrm{MHz}, \mathrm{CDCl} 3$
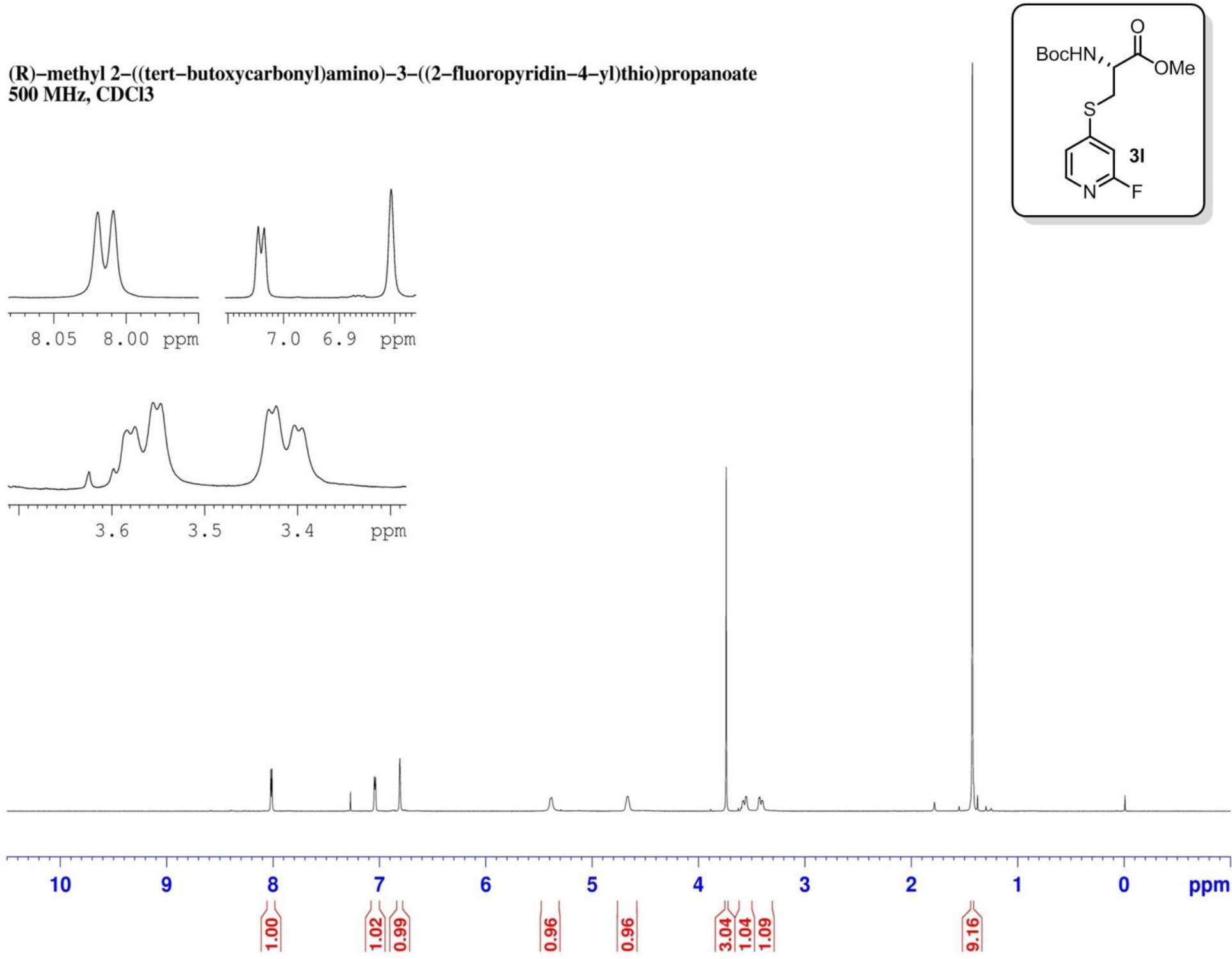
(R)-methyl 2-((tert-butoxycarbonyl)amino)-3-((5-methoxypyridin-3-yl)thio)propanoate $500 \mathrm{MHz}, \mathrm{CDCl} 3$
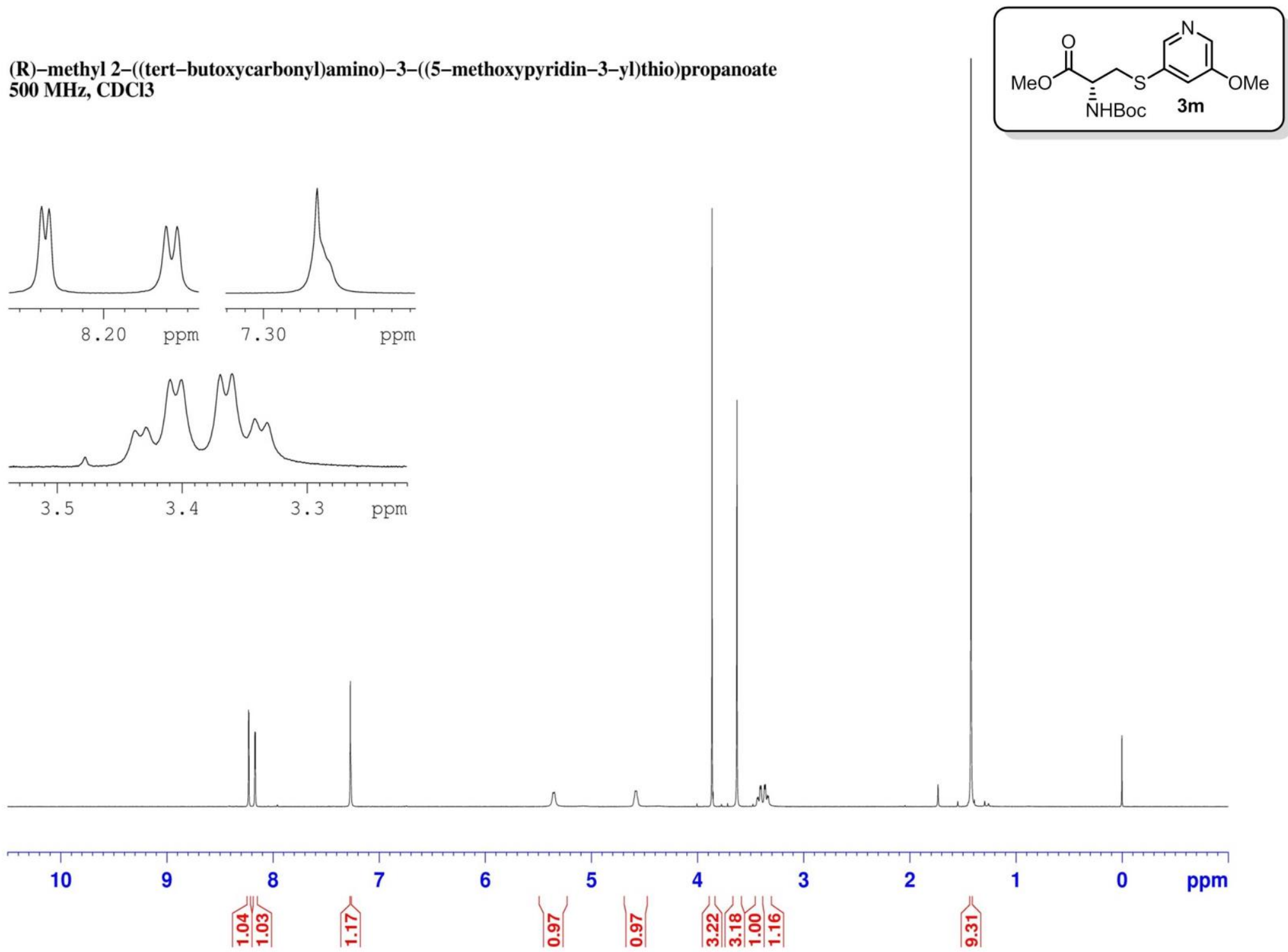
(R)-methyl 2-((tert-butoxycarbonyl)amino)-3-((5-methylpyridin-2-yl)thio)propanoate $500 \mathrm{MHz}, \mathrm{CDCl} 3$
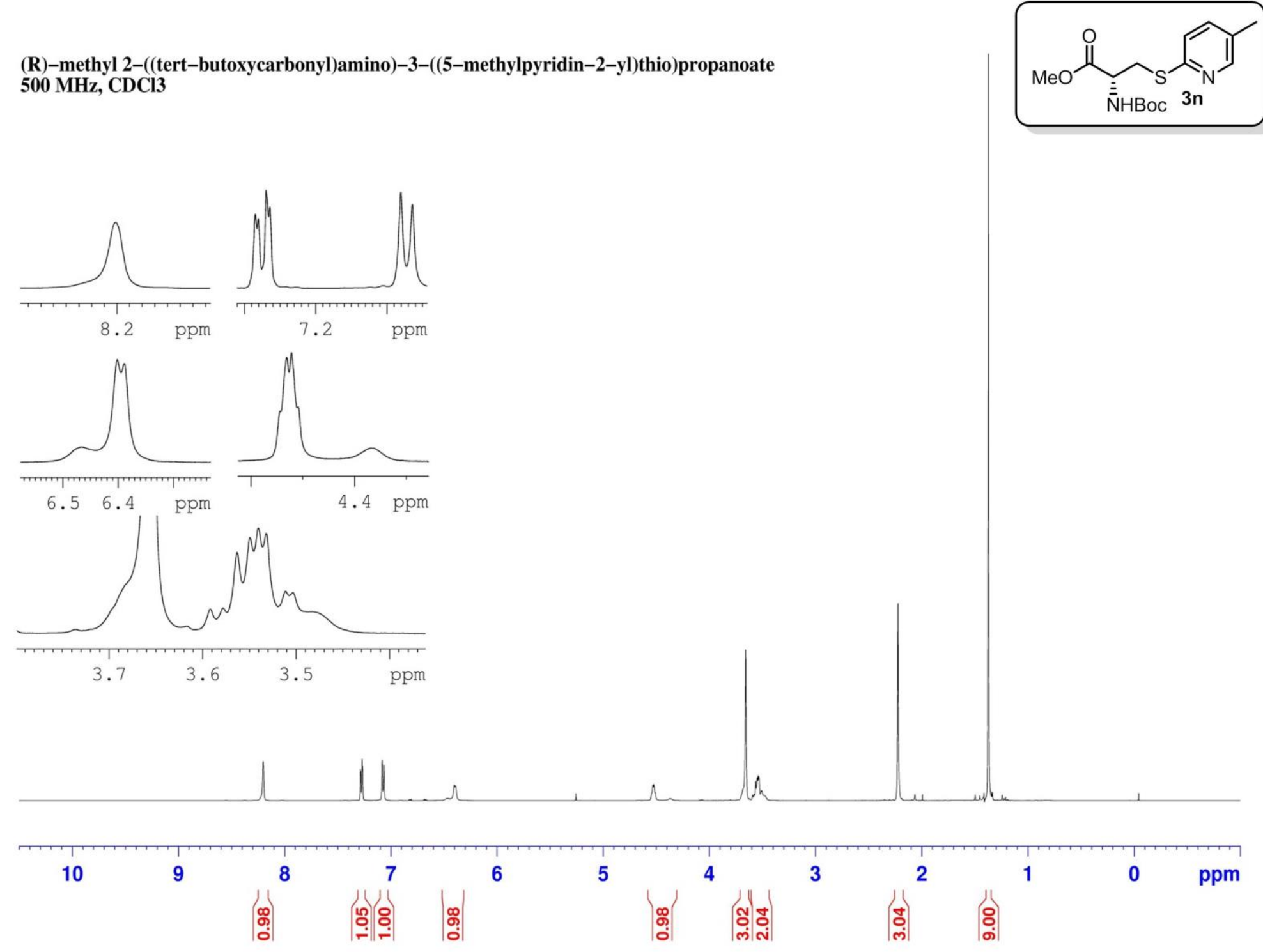
(R)-methyl 2-((tert-butoxycarbonyl)amino)-3-(quinolin-4-ylthio)propanoate $500 \mathrm{MHz}, \mathrm{CDCl} 3$
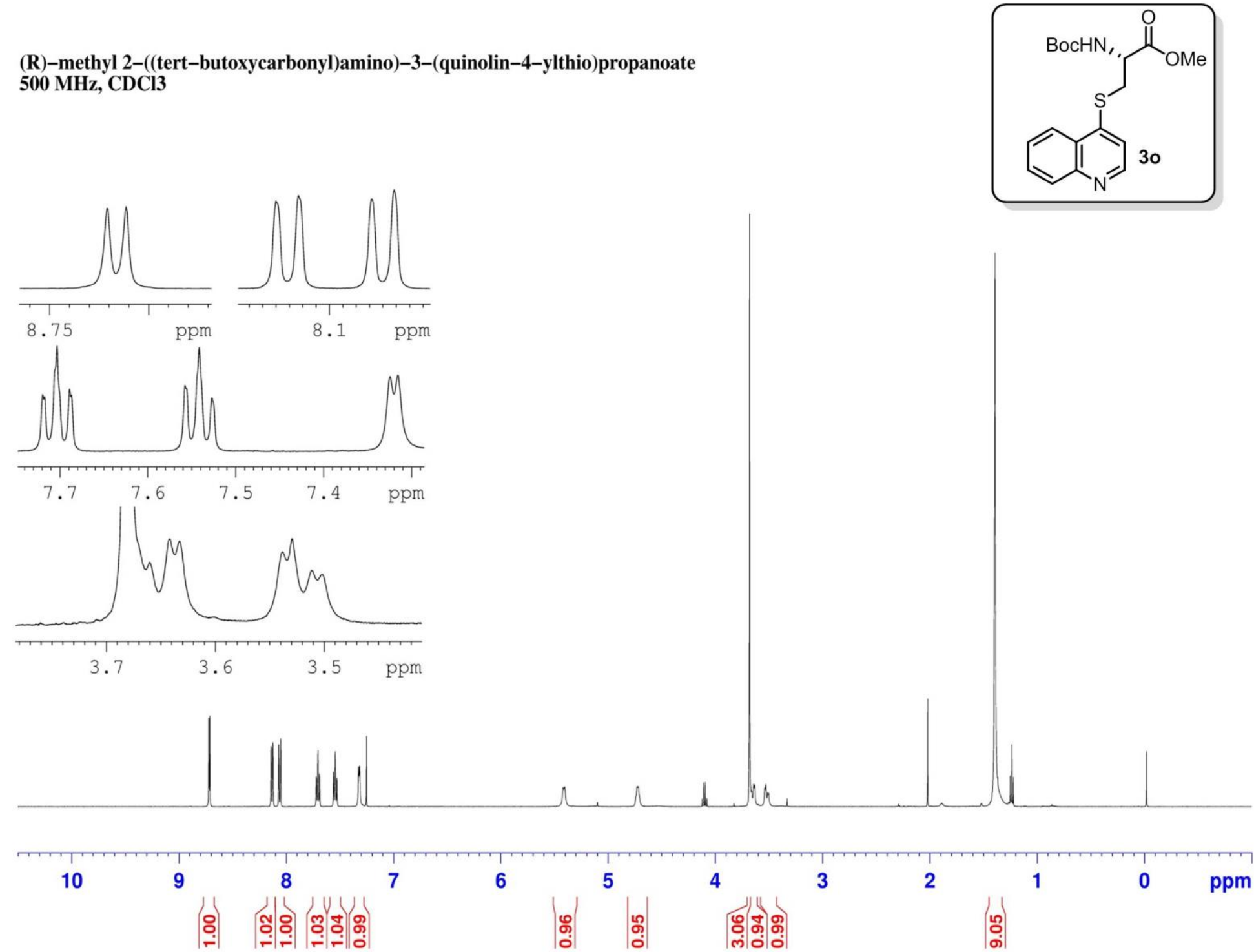
(R)-methyl 2-((tert-butoxycarbonyl)amino)-3-(isoquinolin-4-ylthio)propanoate $500 \mathrm{MHz}, \mathrm{CDCl} 3$
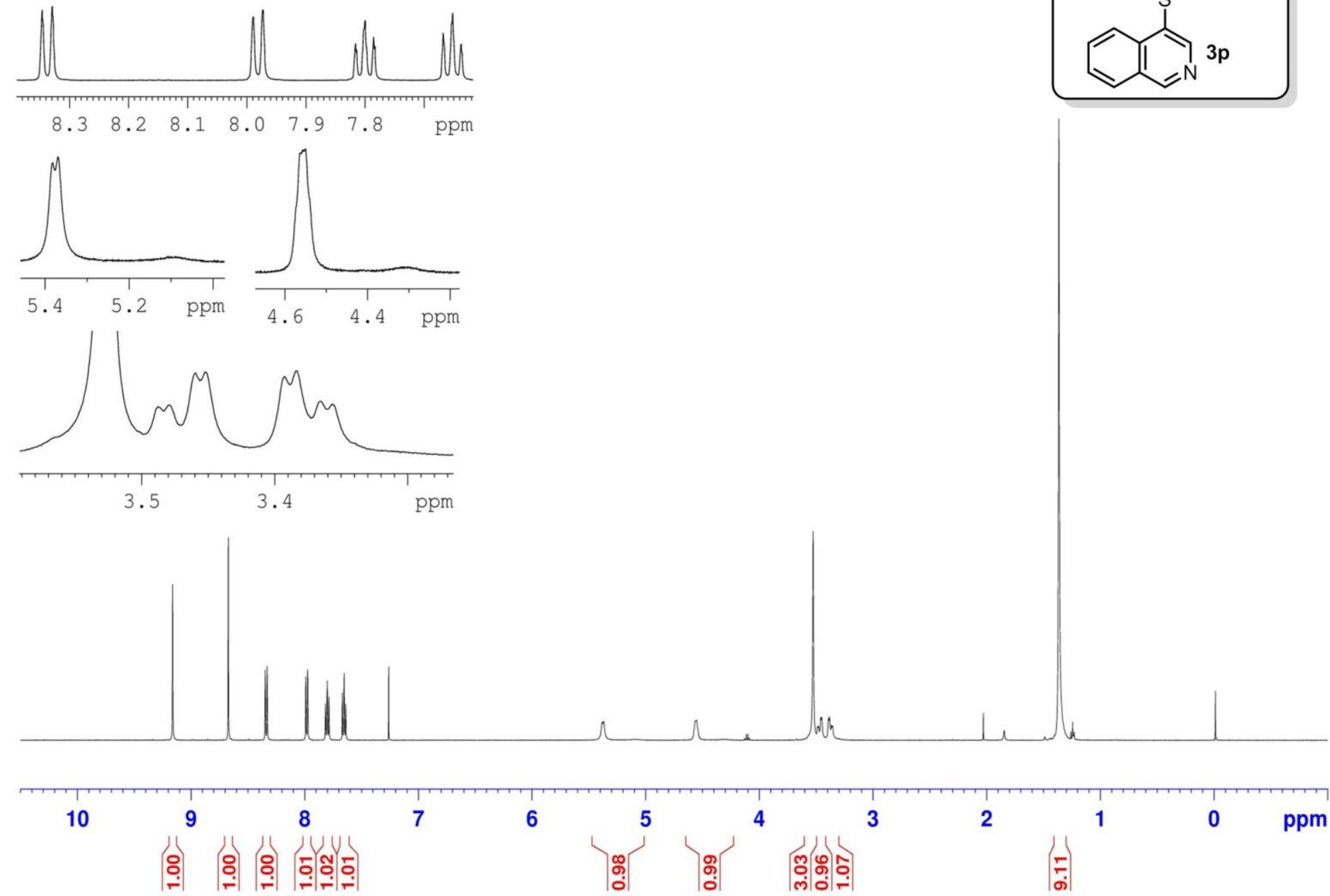
3-(pyrimidin-5-ylthio)hexan-1-ol $500 \mathrm{MHz}, \mathrm{CDCl} 3$
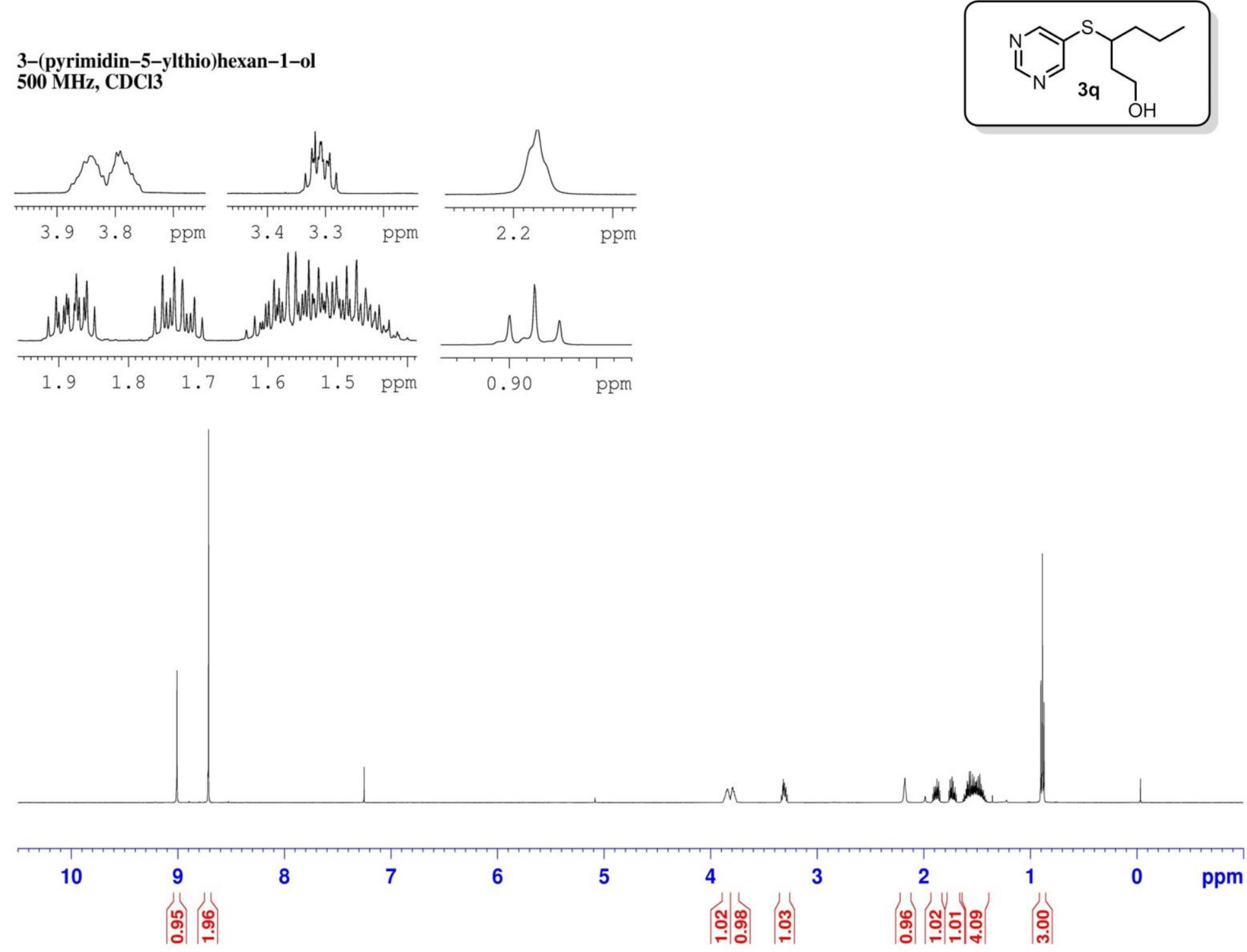
tert-butyl 4-(5-((1-hydroxyhexan-3-yl)thio)pyrimidin-2-yl)piperazine-1-carboxylate $500 \mathrm{MHz}, \mathrm{CDCl} 3$
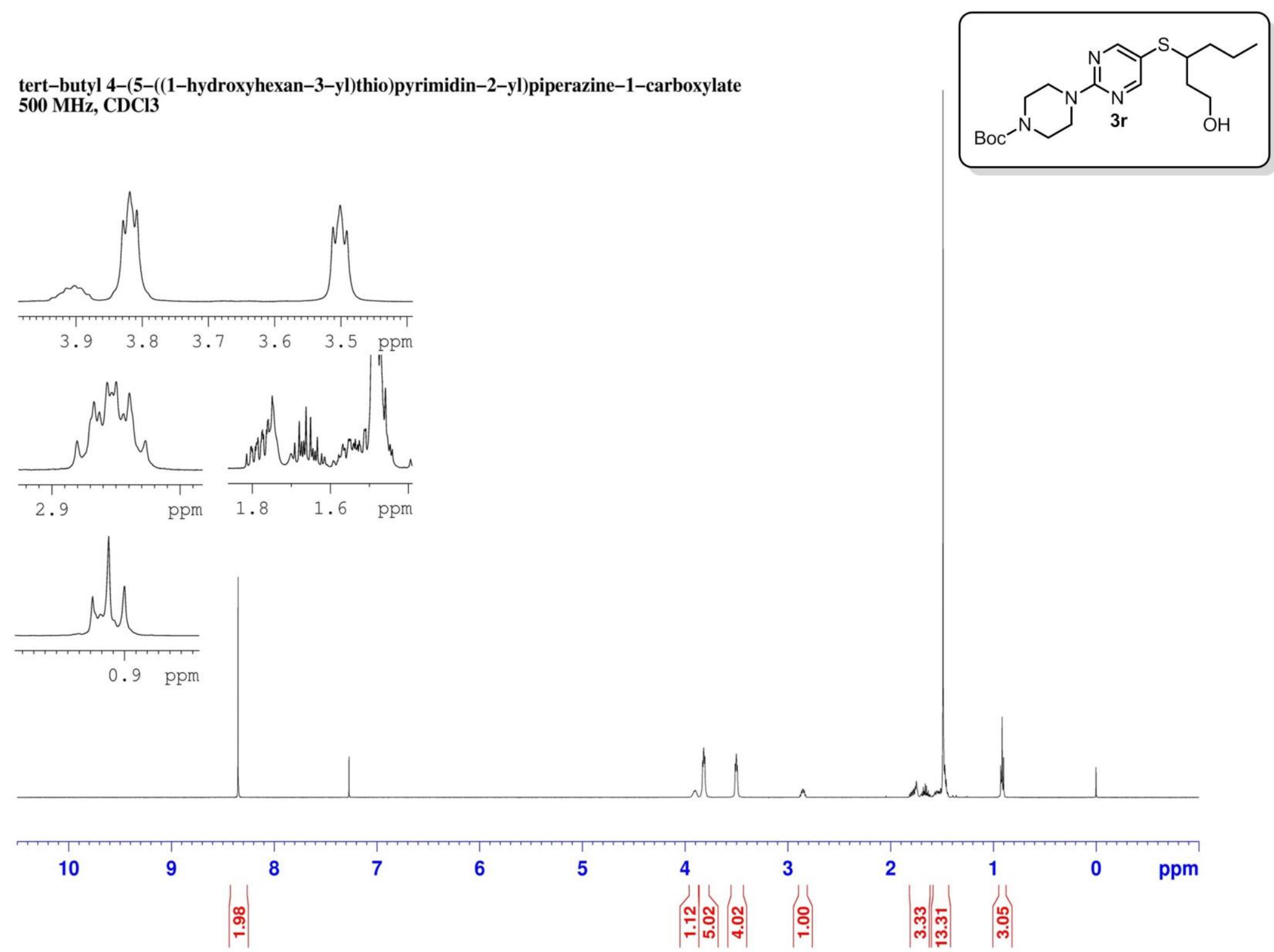
3-((3-methylpyridin-2-yl)thio)hexan-1-ol

$500 \mathrm{MHz}, \mathrm{CDCl} 3$
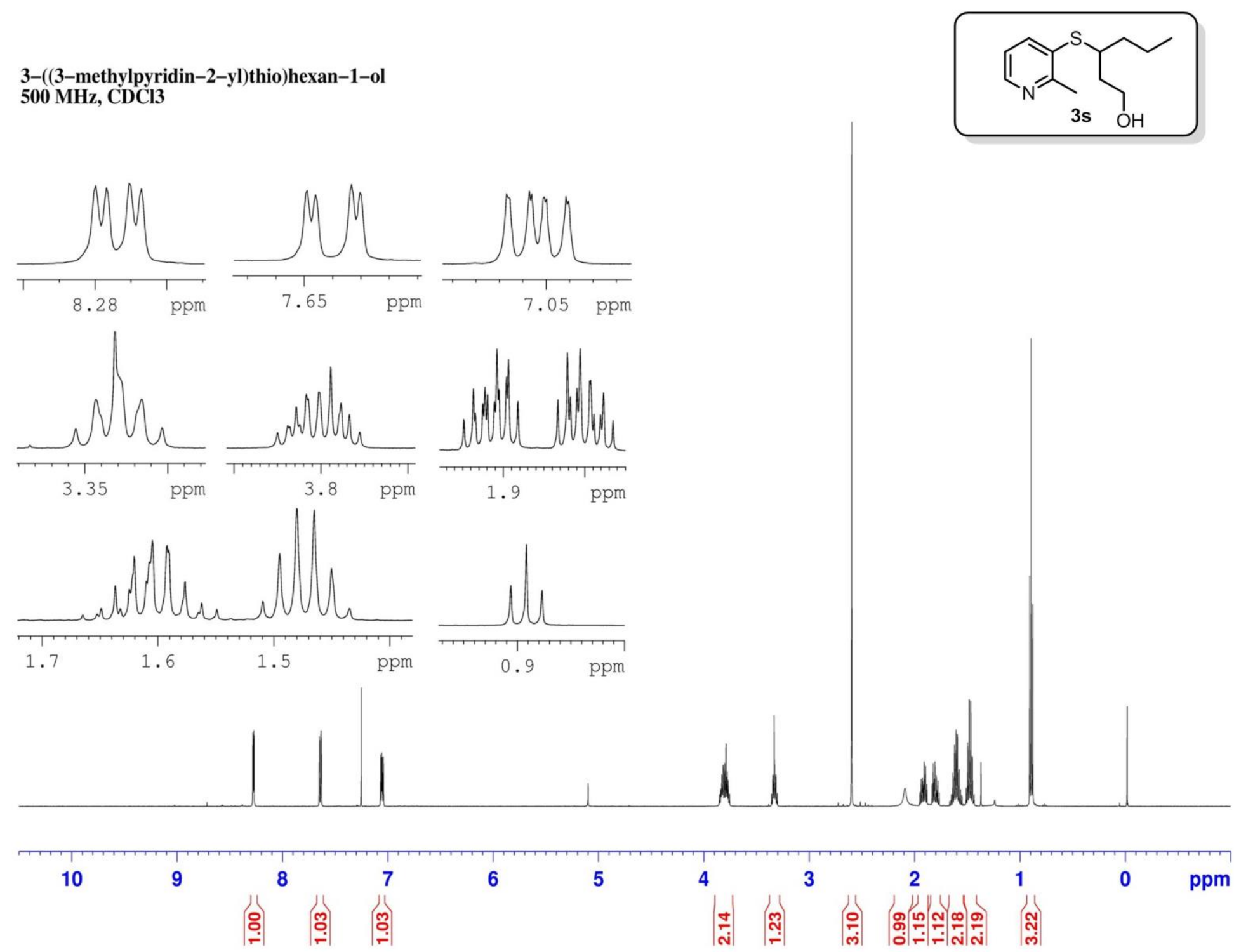
N-(4-((1-hydroxyhexan-3-yl)thio)phenyl)acetamide $500 \mathrm{MHz}, \mathrm{CDCl} 3$
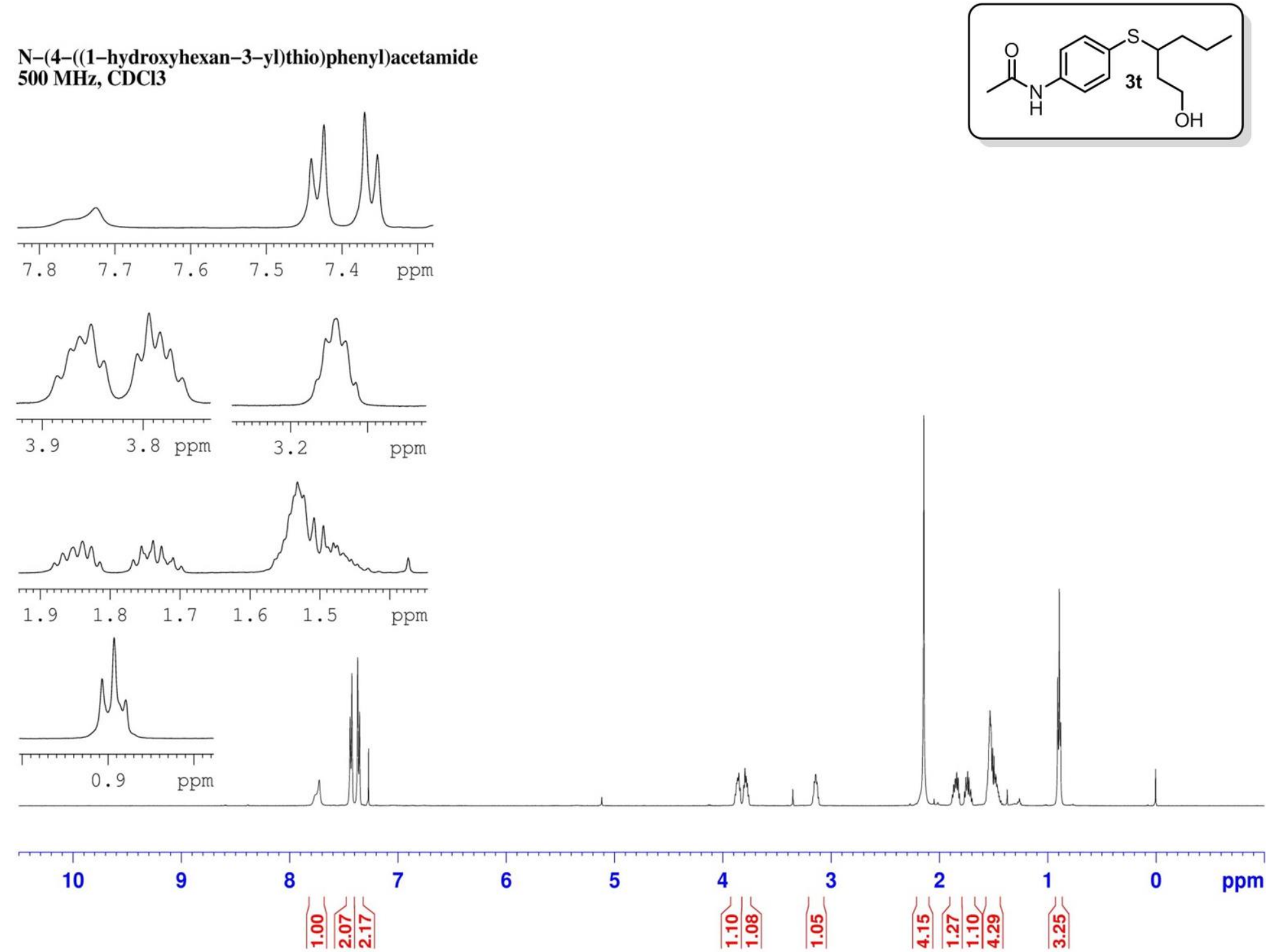
4-((1-hydroxyhexan-3-yl)thio)benzenesulfonamide $500 \mathrm{MHz}, \mathrm{CDCl} 3$
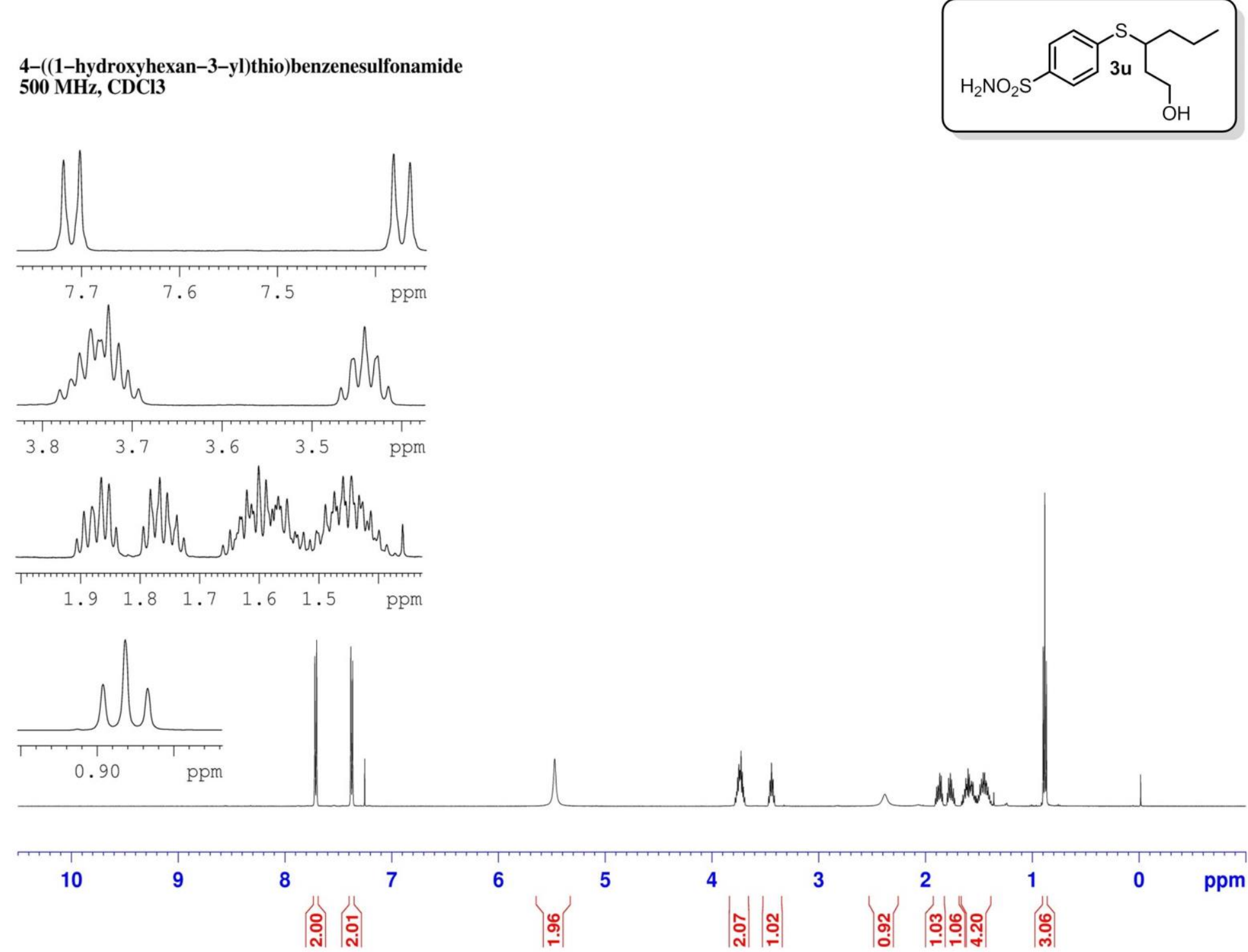
4-(tert-butylthio)benzoic acid

$500 \mathrm{MHz}, \mathrm{CDCl} 3$
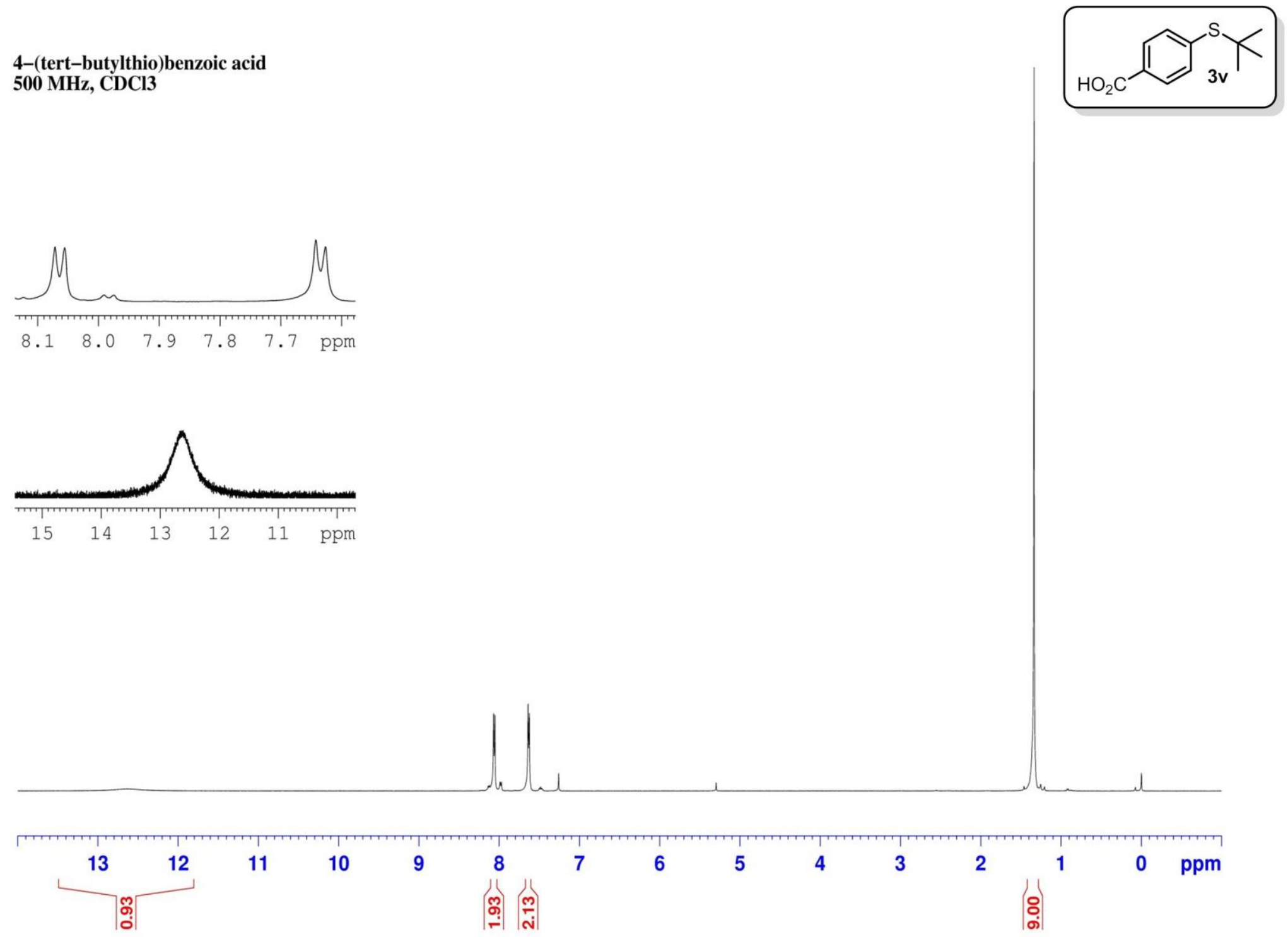
(4-benzylphenyl)(tert-butyl)sulfane $500 \mathrm{MHz}, \mathrm{CDCl} 3$
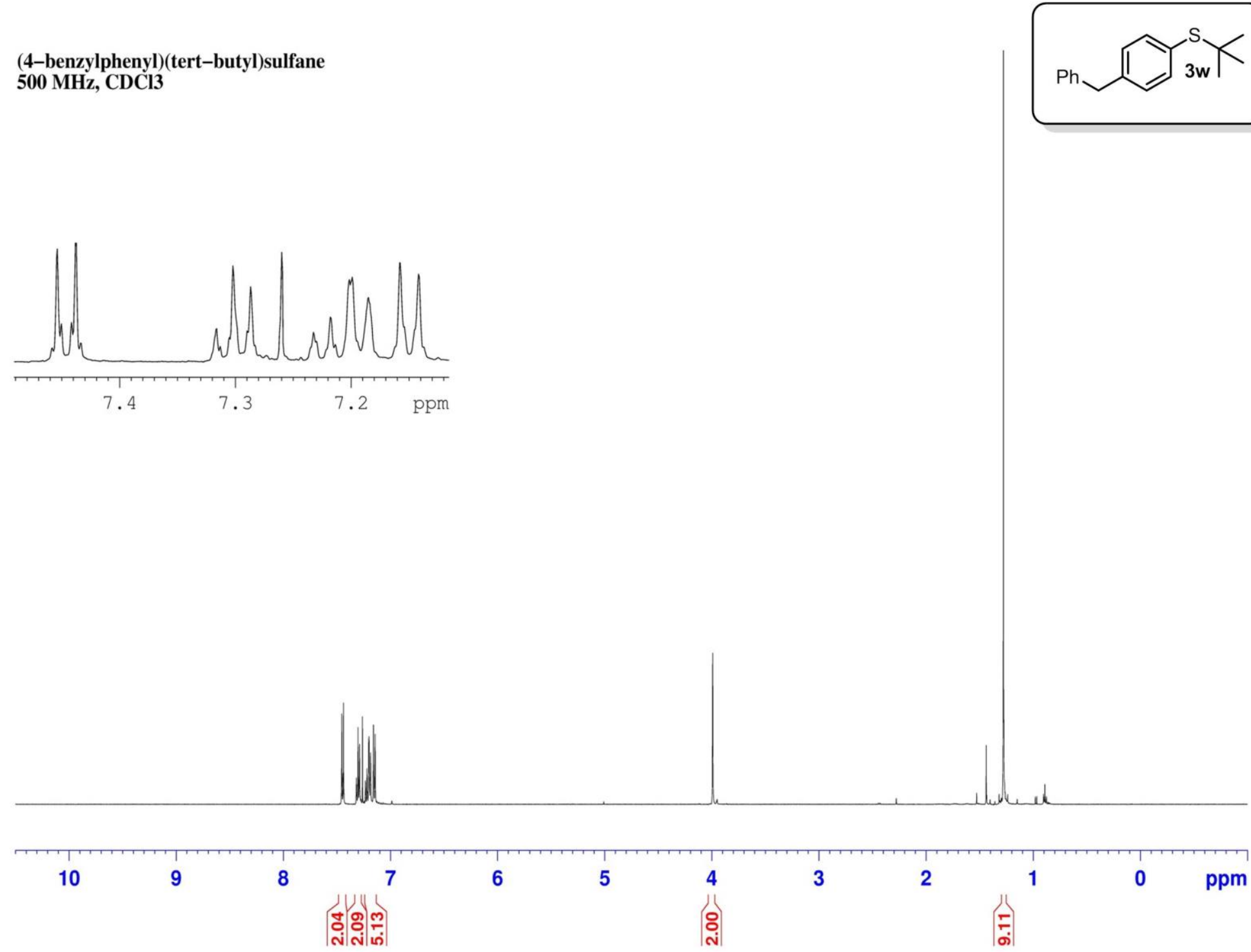
2-(tert-butylthio)benzonitrile $500 \mathrm{MHz}, \mathrm{CDCl} 3$

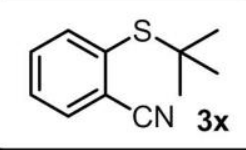

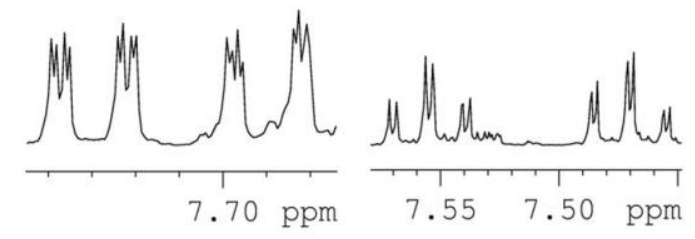

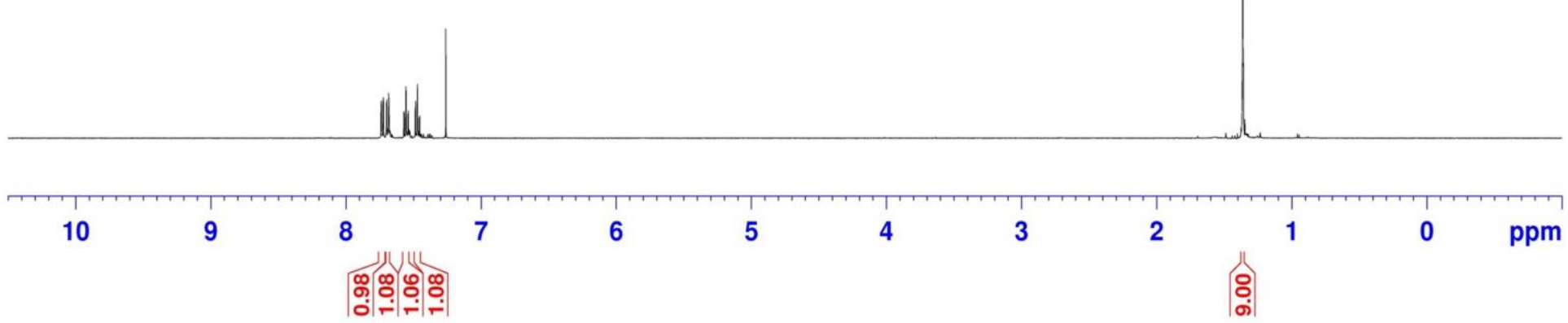


3-(tert-butylthio)-5-chloropyridine $500 \mathrm{MHz}, \mathrm{CDCl} 3$
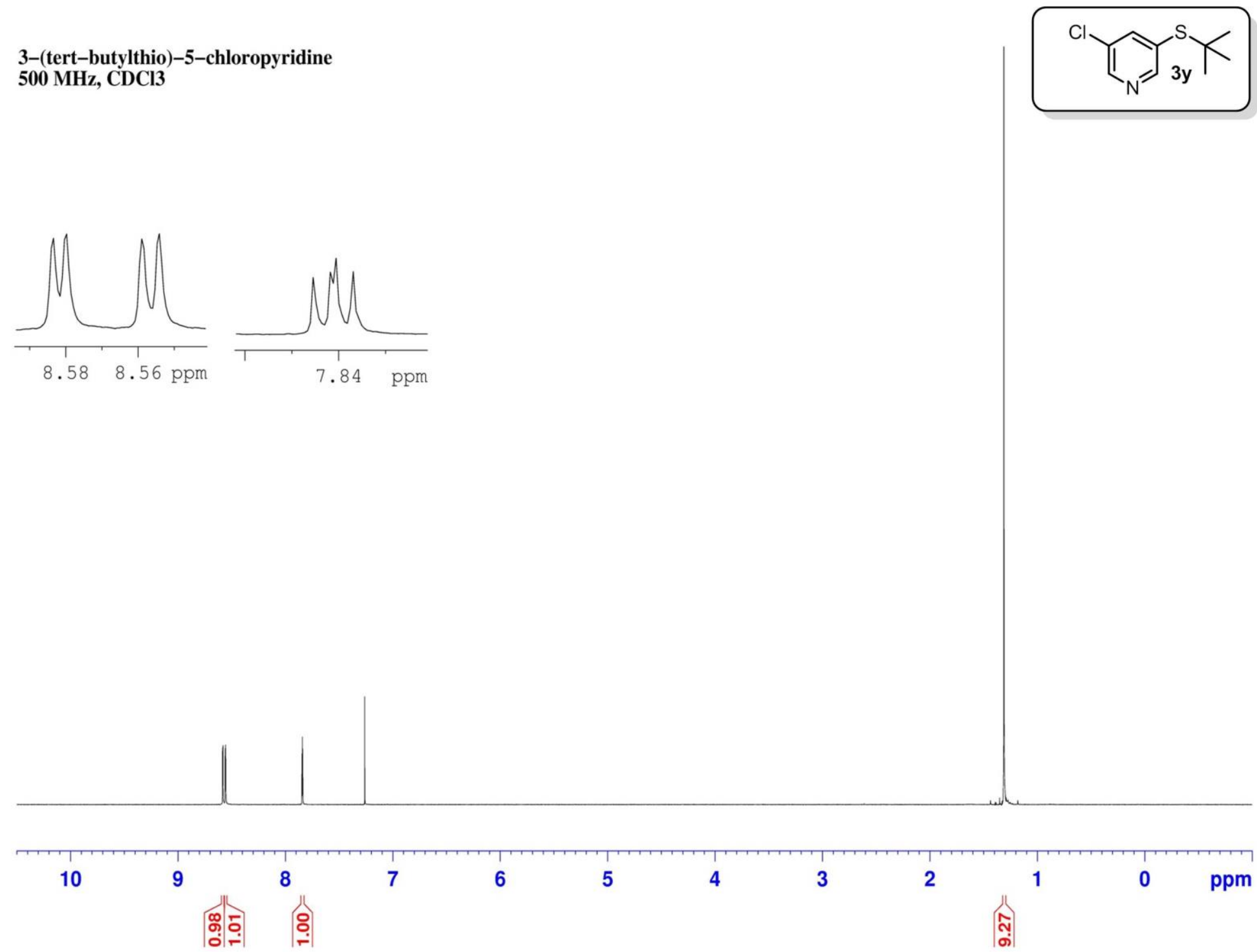
8-(tert-butylthio)isoquinoline

$500 \mathrm{MHz}, \mathrm{CDCl} 3$
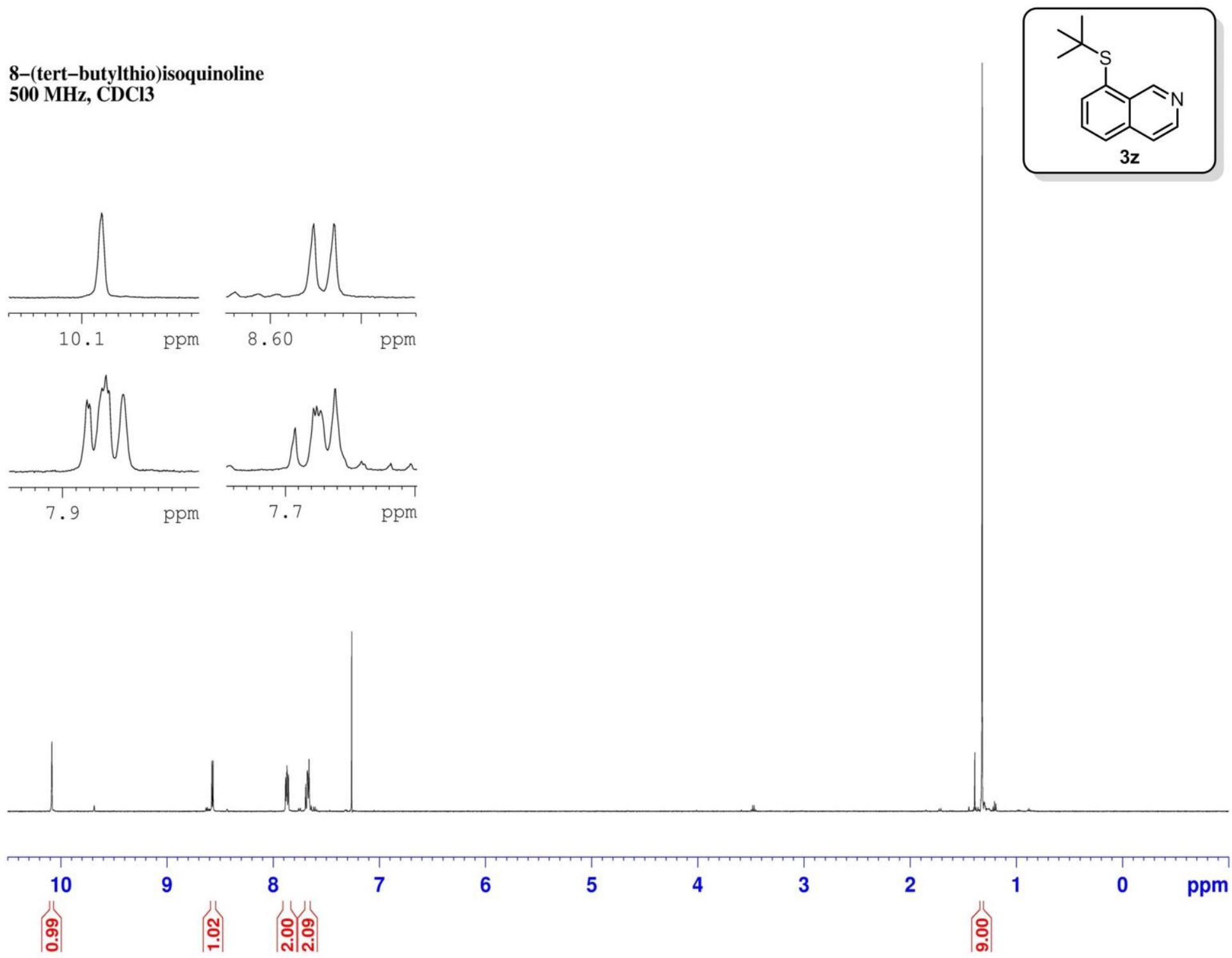


\section{${ }^{13}$ C NMR Spectra of Synthesized Compounds}

diisopropylammonium bis(catecholato)(3-mercaptopropyl)silicate $125 \mathrm{MHz}, \mathrm{MeCN}-\mathrm{d} 3$
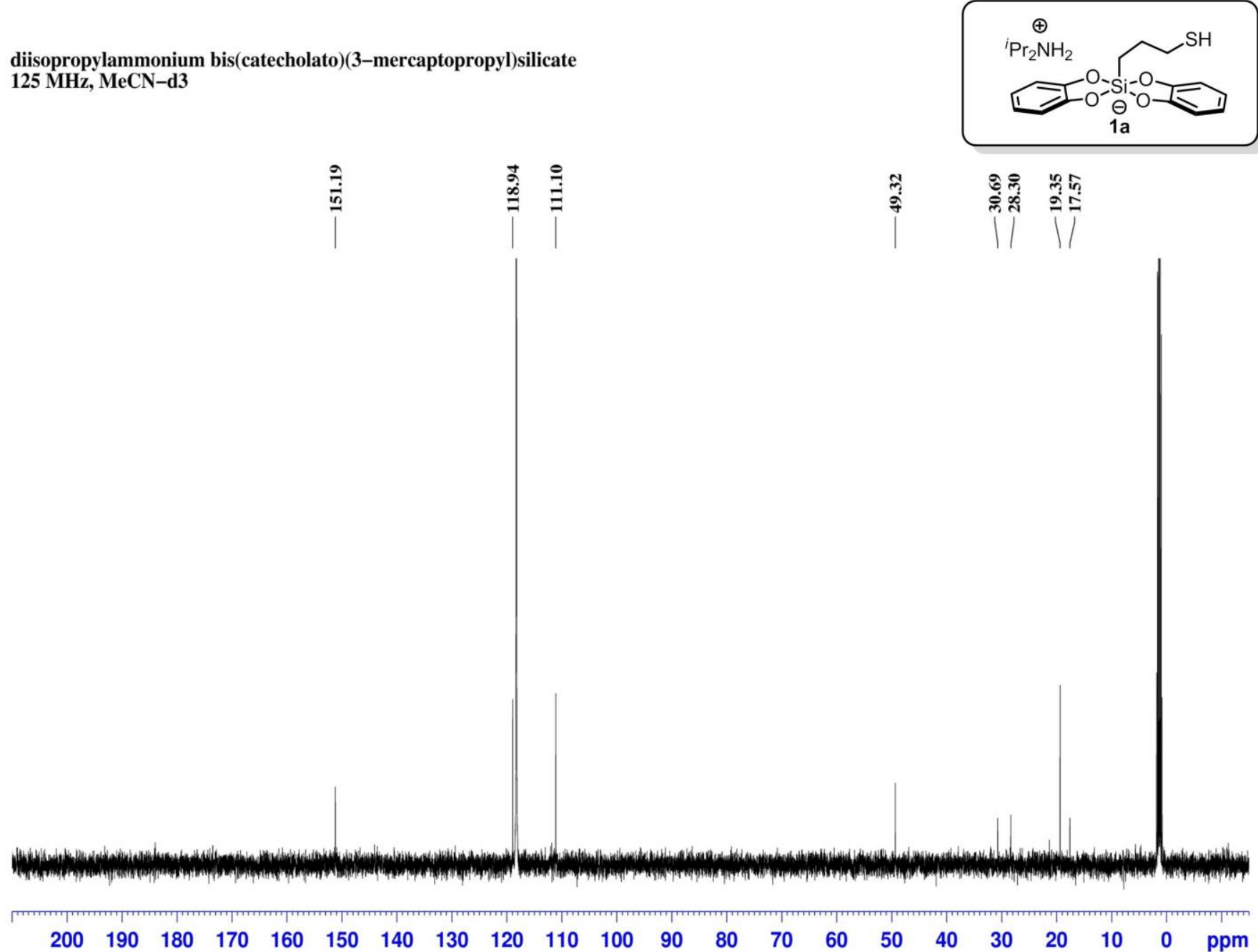
diisopropylammonium bis(catecholato)isobutylsilicate 125 MHz, DMSO-d6
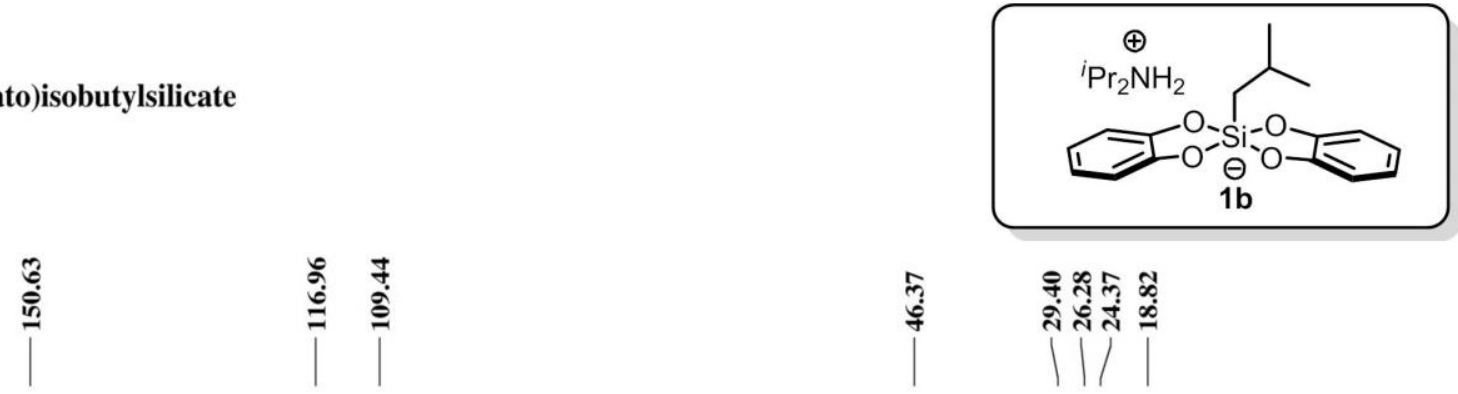

ำ

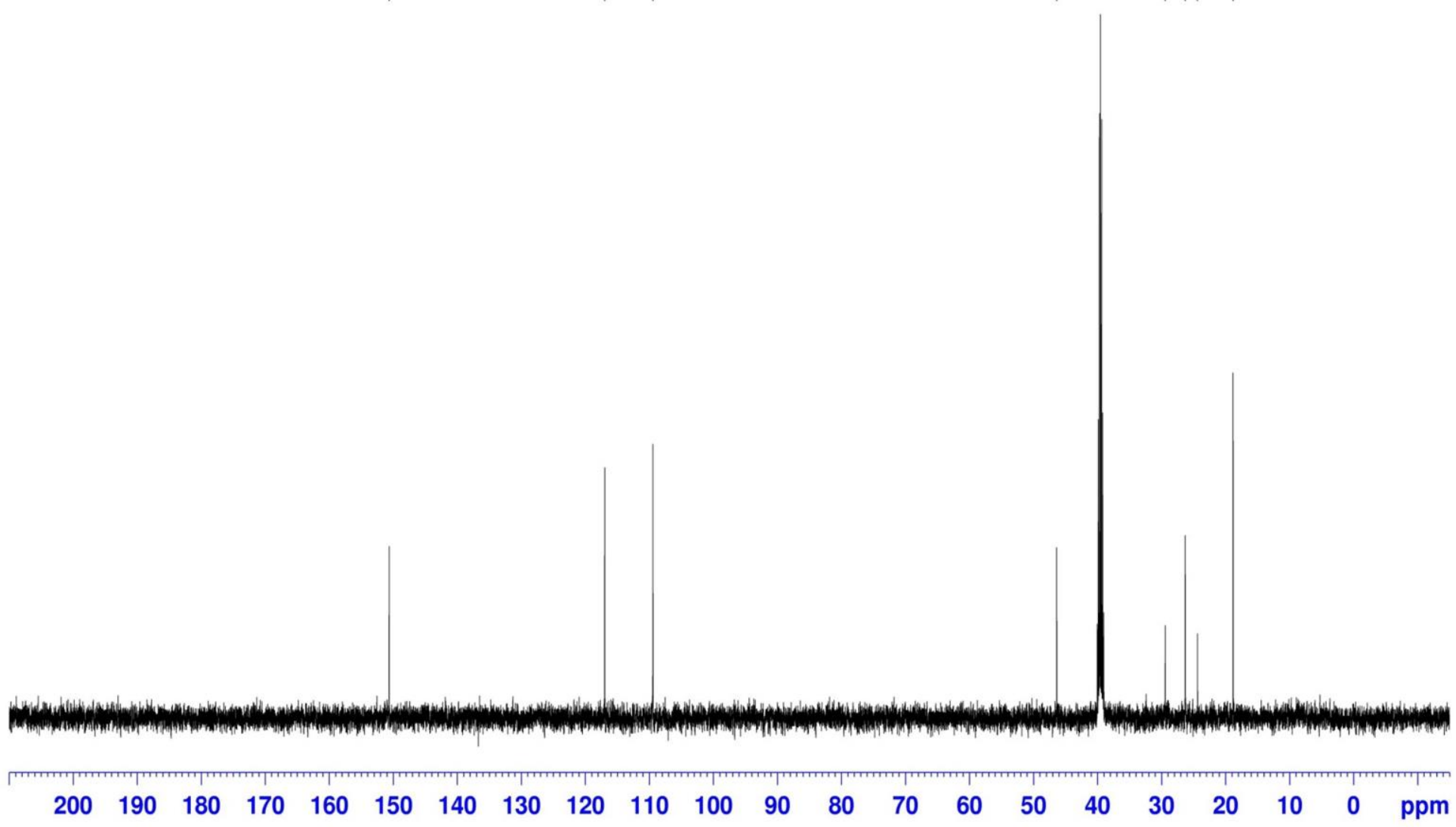


diisopropylammonium bis(catecholato)hexadecylsilicate 125 MHz, DMSO-d6
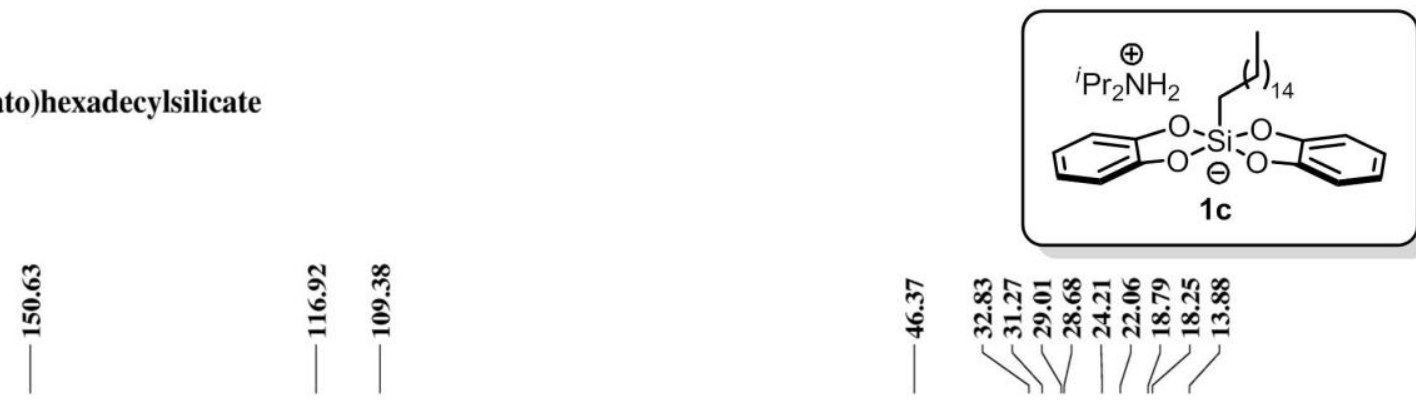

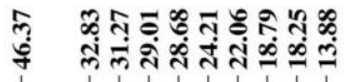

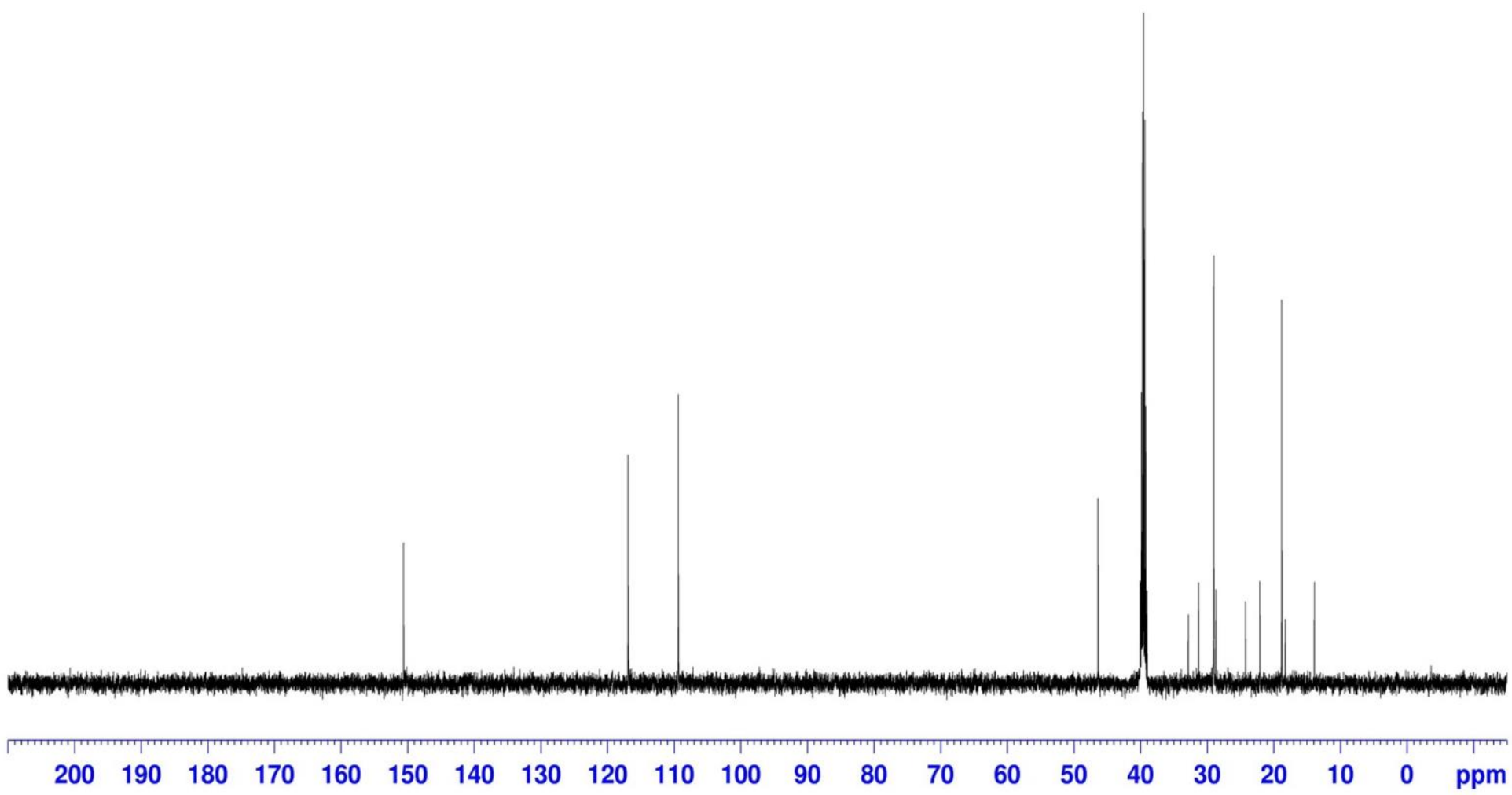


[1,1'-biphenyl]-4-yl(propyl)sulfane

$125 \mathrm{MHz}, \mathrm{CDCl} 3$
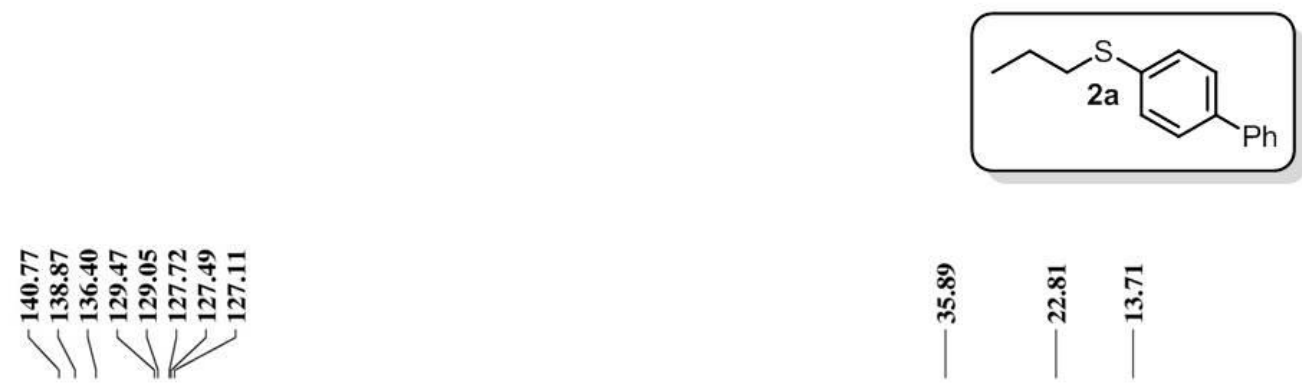

ஸे

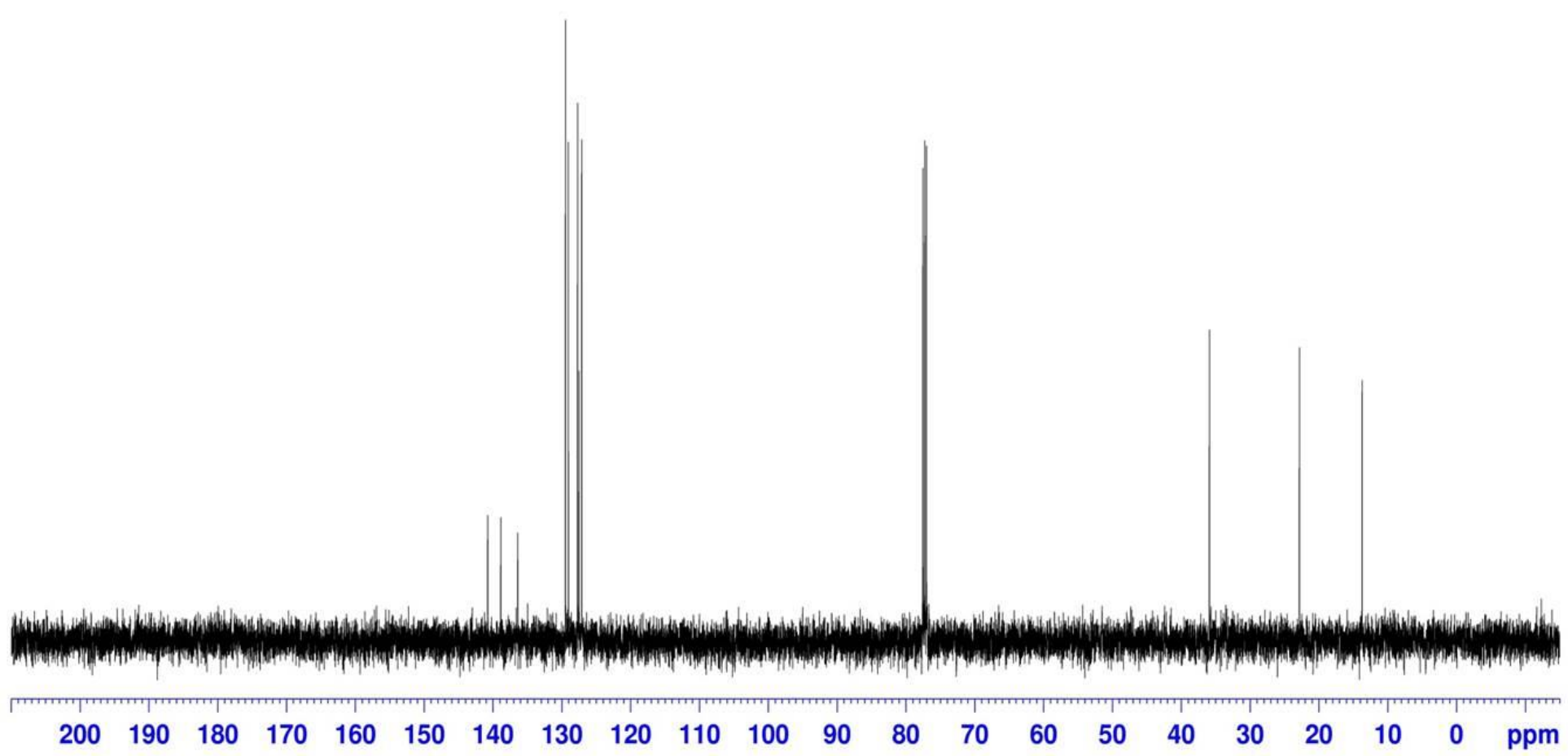


(4-benzylphenyl)(propyl)sulfane

$125 \mathrm{MHz}, \mathrm{CDCl} 3$
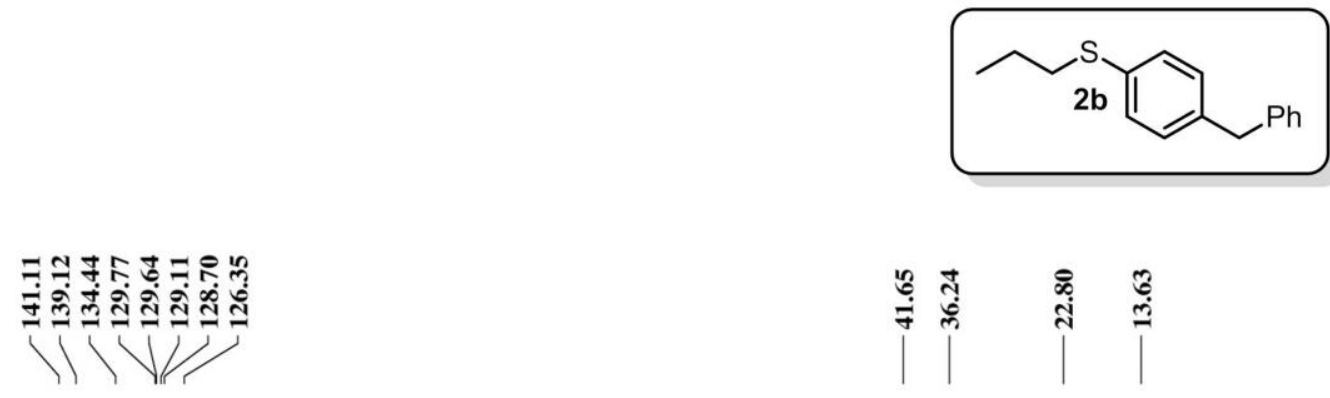

|

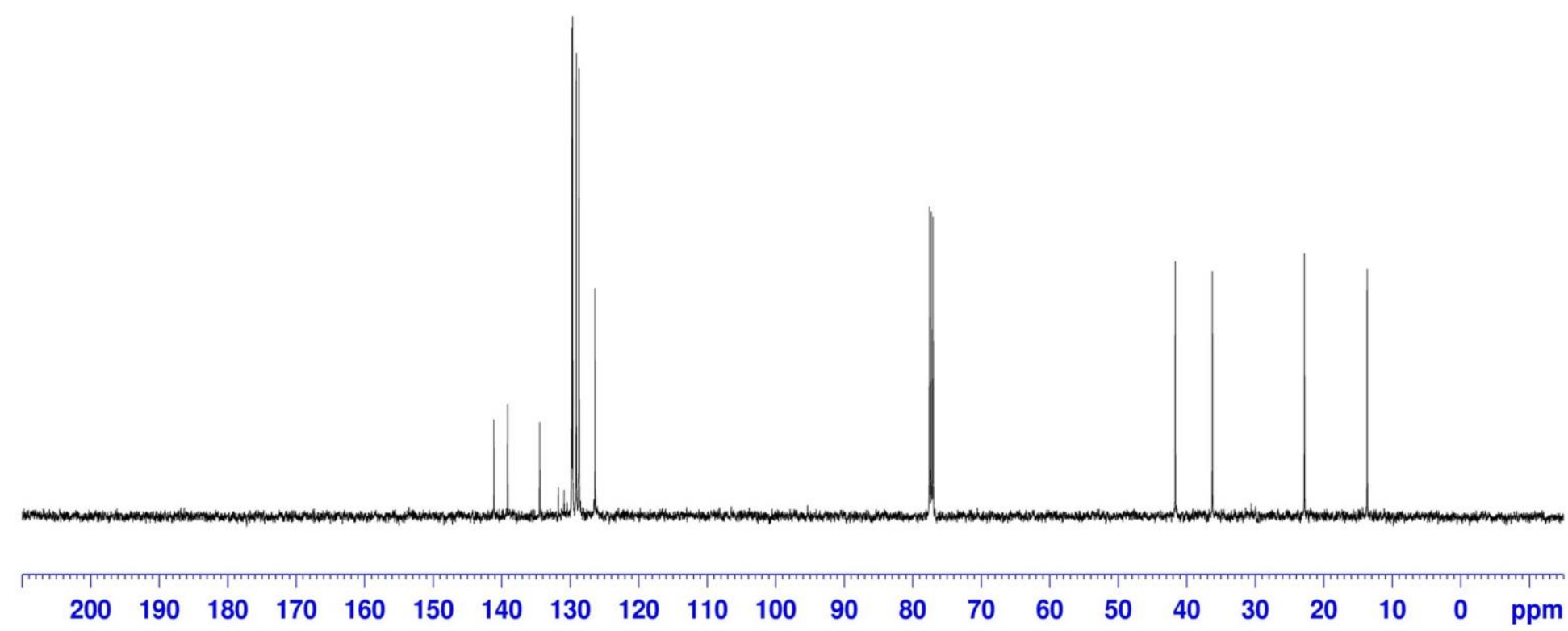


4-(propylthio)benzonitrile

$125 \mathrm{MHz}, \mathrm{CDCl} 3$
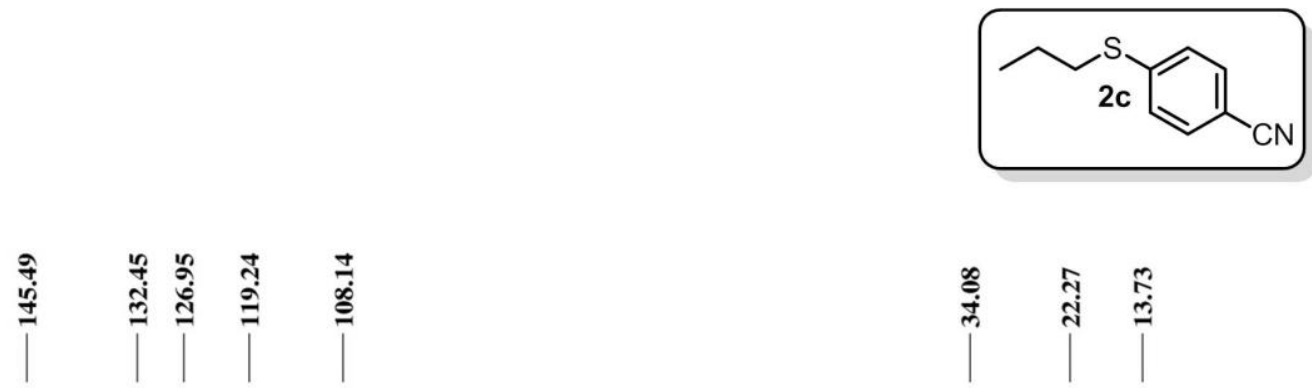

|

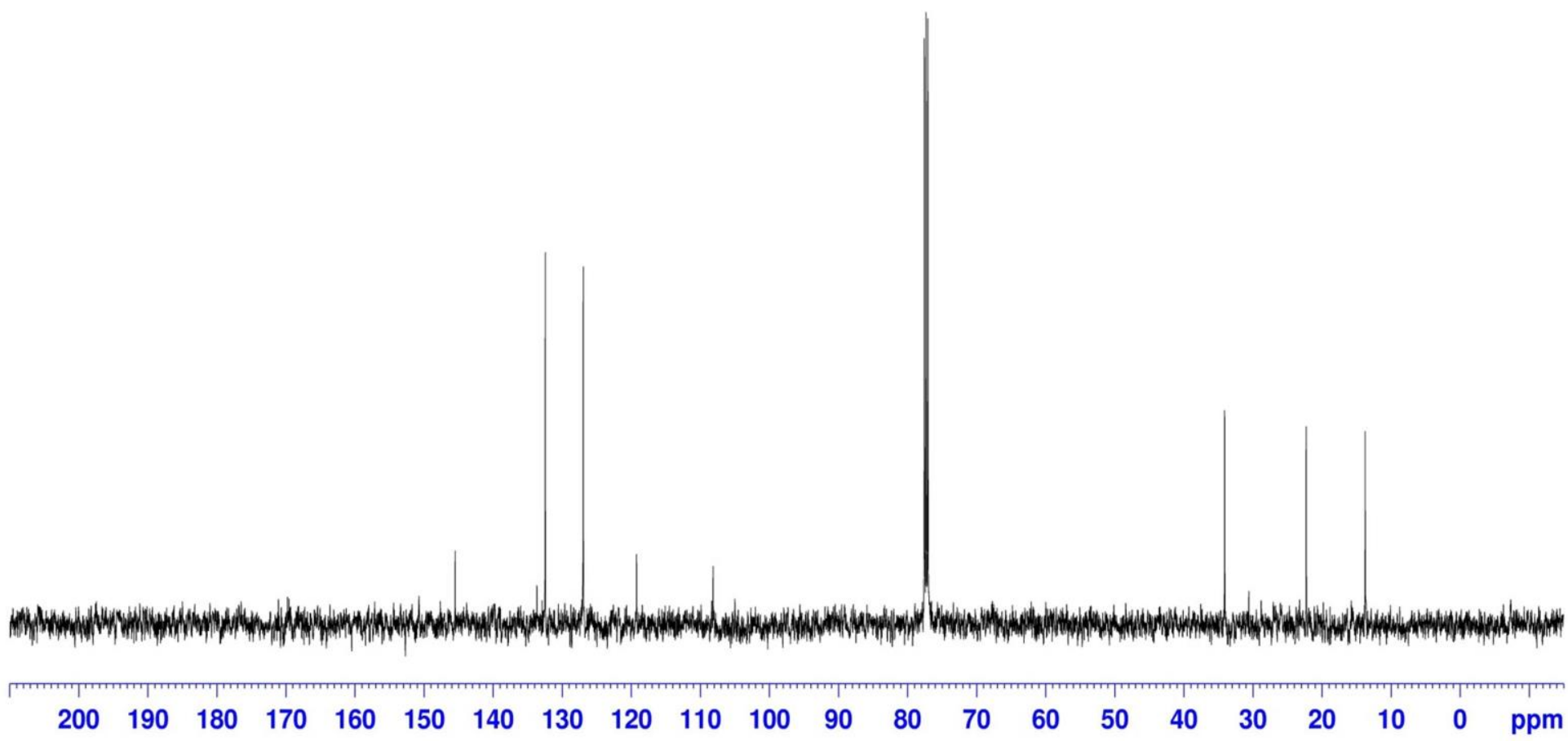


1-(4-(propylthio)phenyl)ethanone

$125 \mathrm{MHz}, \mathrm{CDCl} 3$
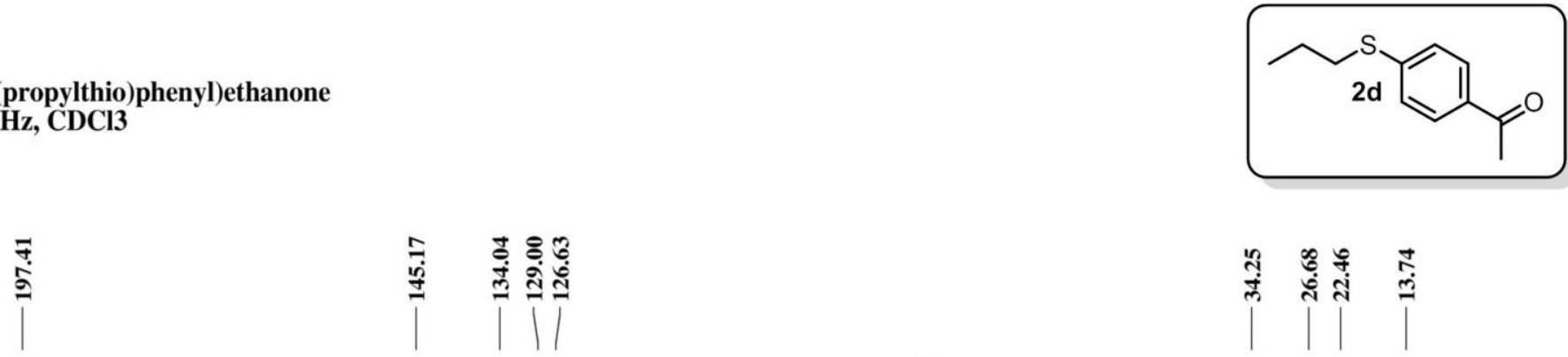

๓ึ

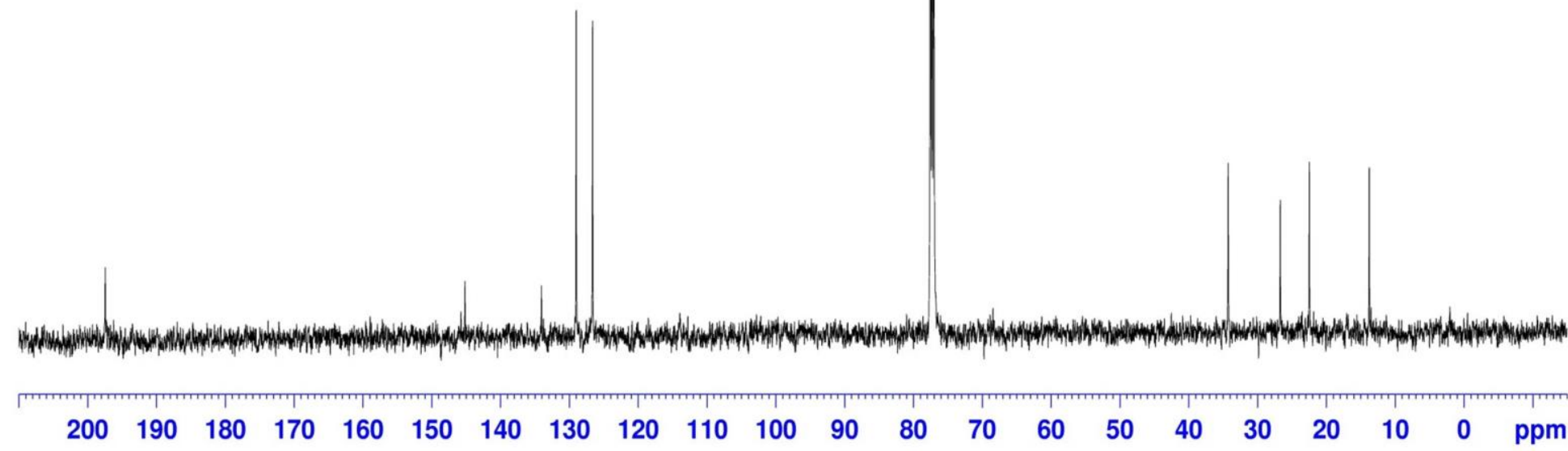


2-(propylthio)benzonitrile

$125 \mathrm{MHz}, \mathrm{CDCl} 3$
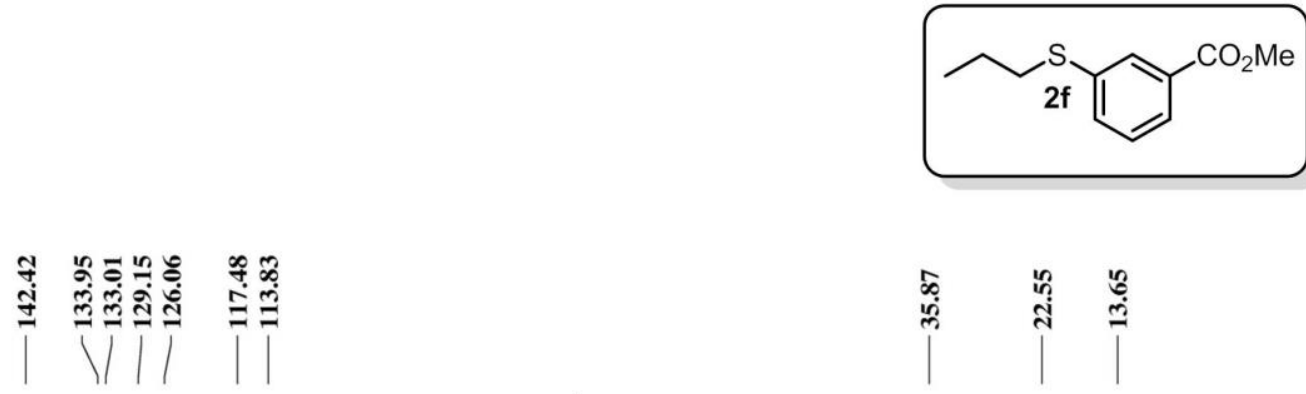

|

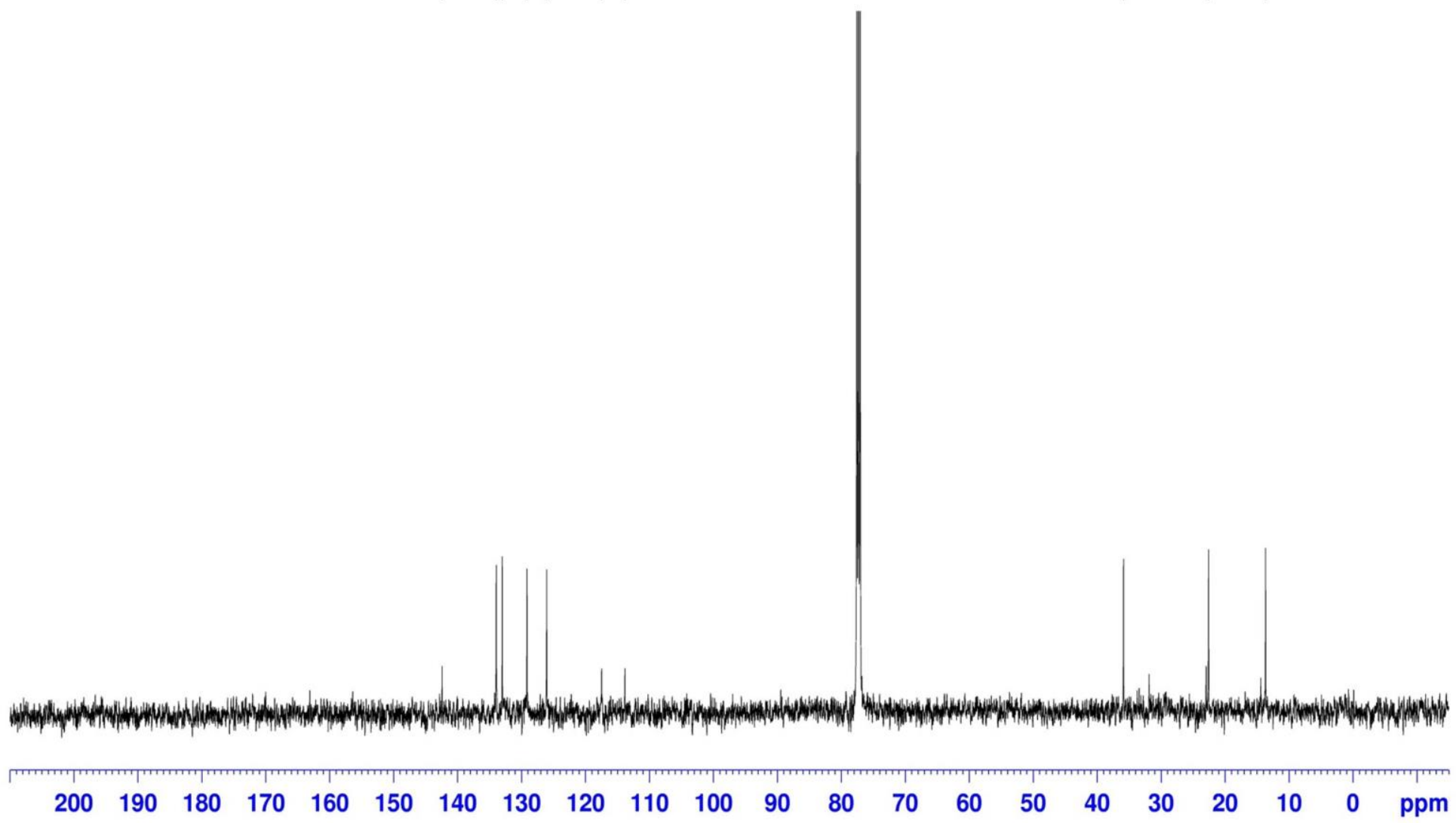



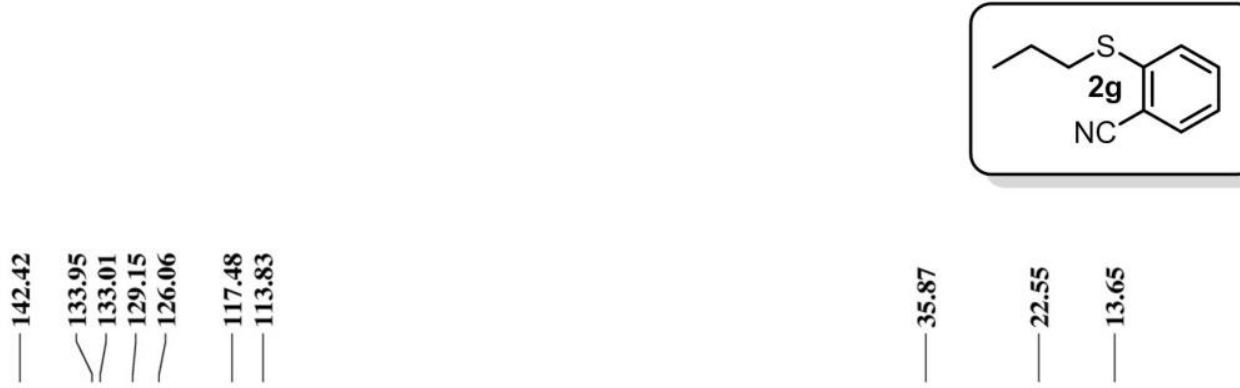

|

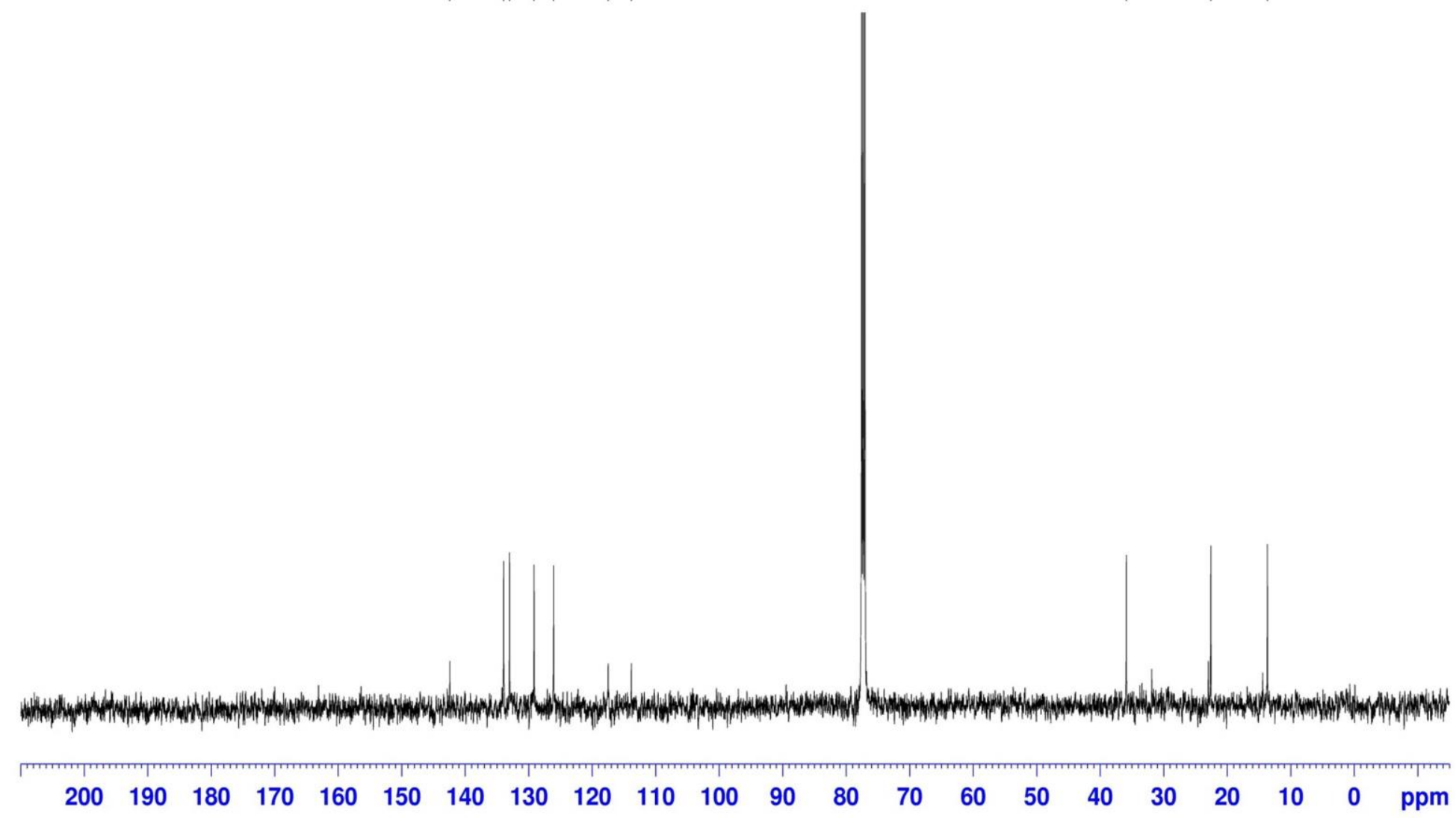


4-(propylthio)benzoic acid

$125 \mathrm{MHz}, \mathrm{CDCl} 3$
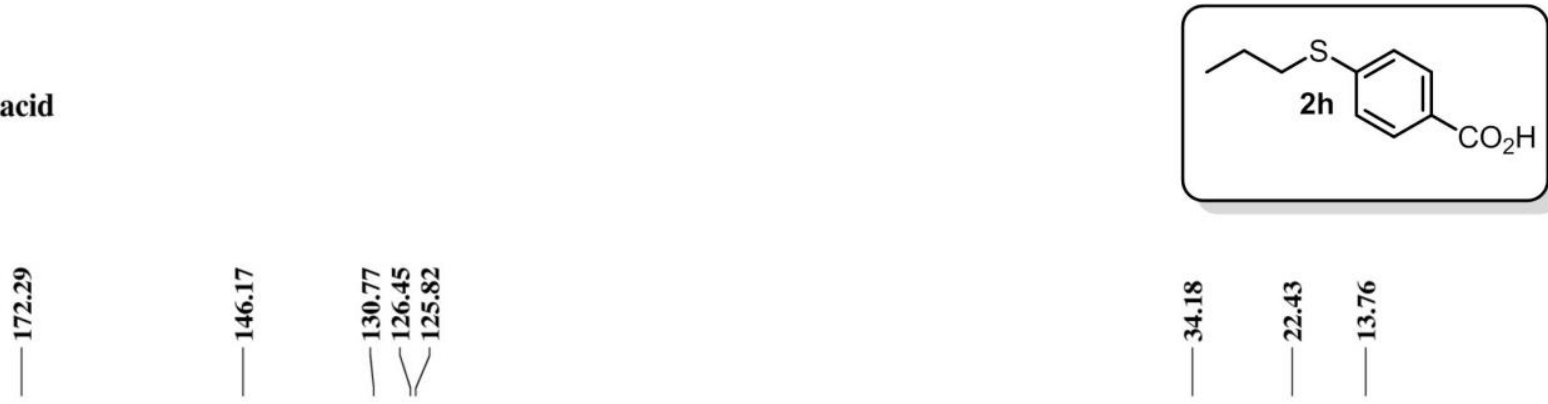

|

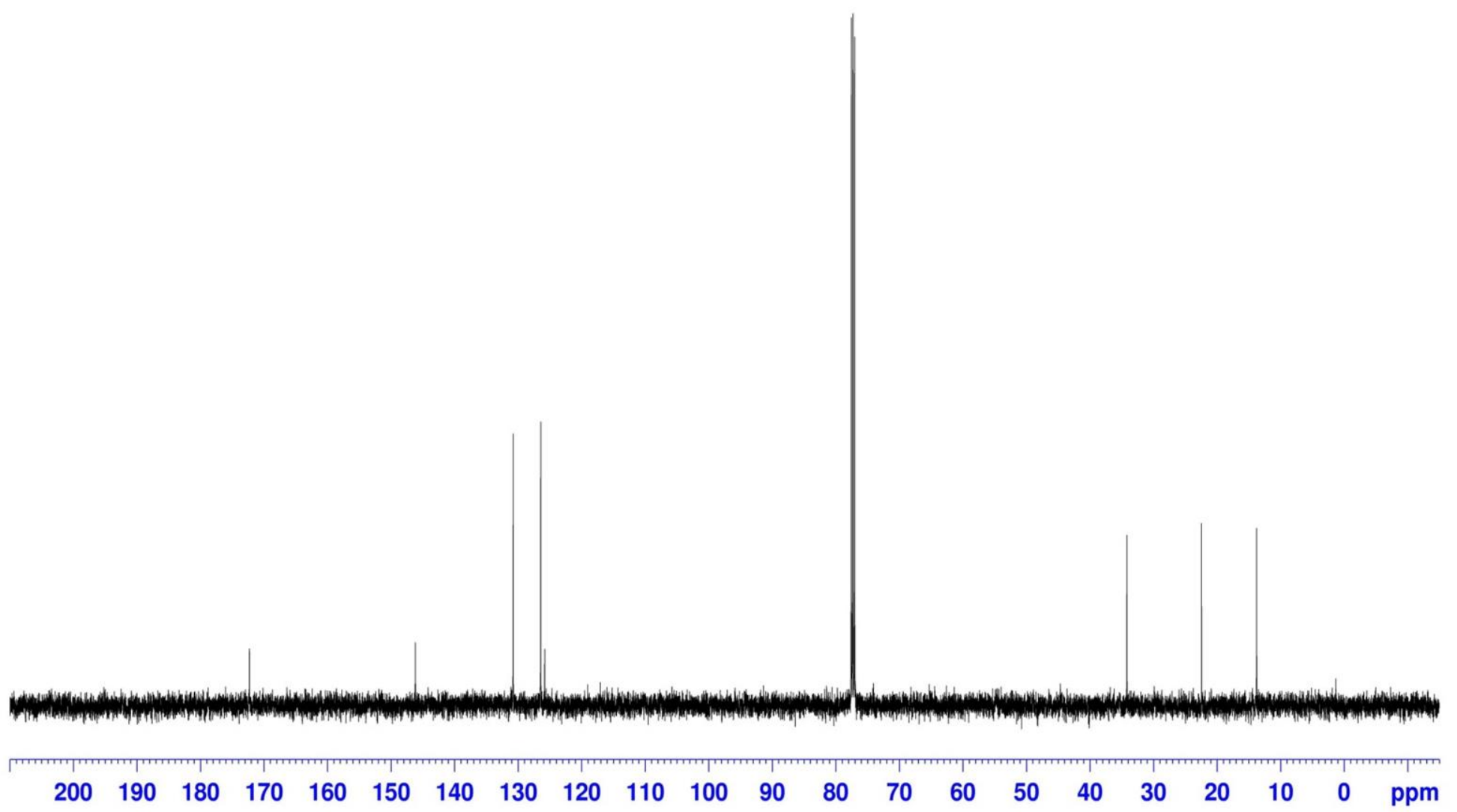


1-(5-(propylthio)thiophen-2-yl)ethanone $125 \mathrm{MHz}, \mathrm{CDCl} 3$
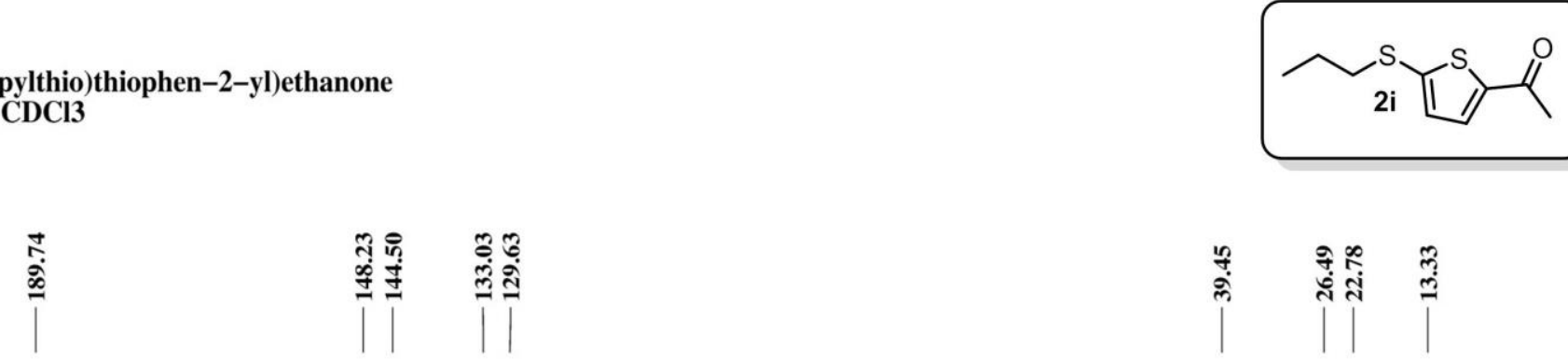

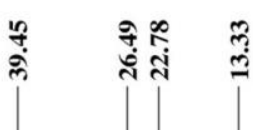

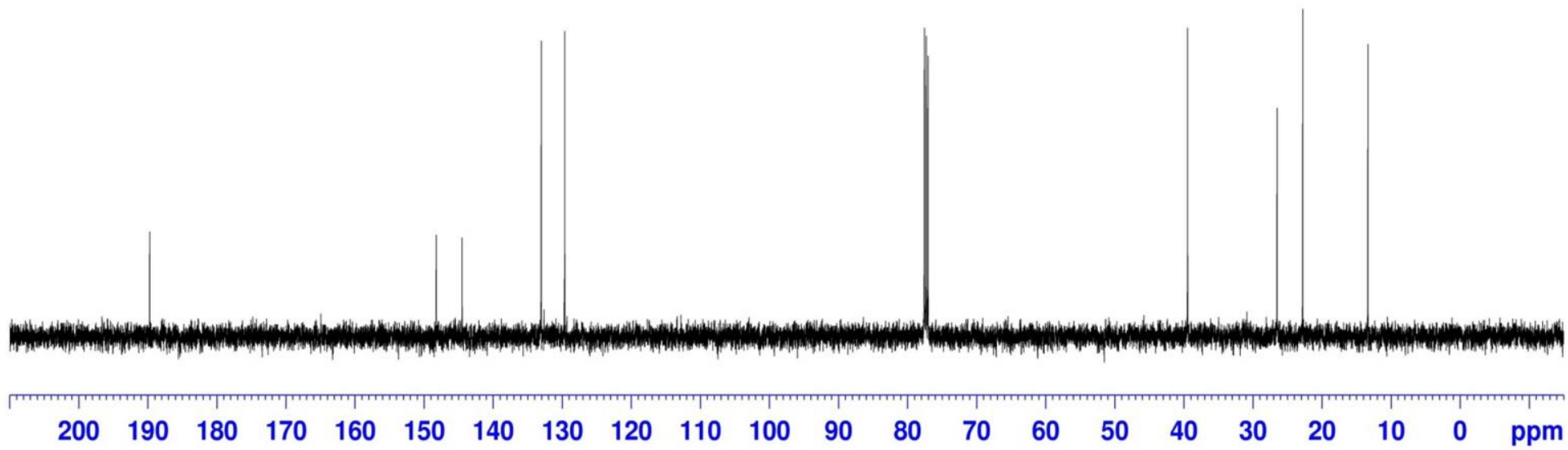


2-fluoro-4-(propylthio)pyridine $125 \mathrm{MHz}, \mathrm{CDCl} 3$
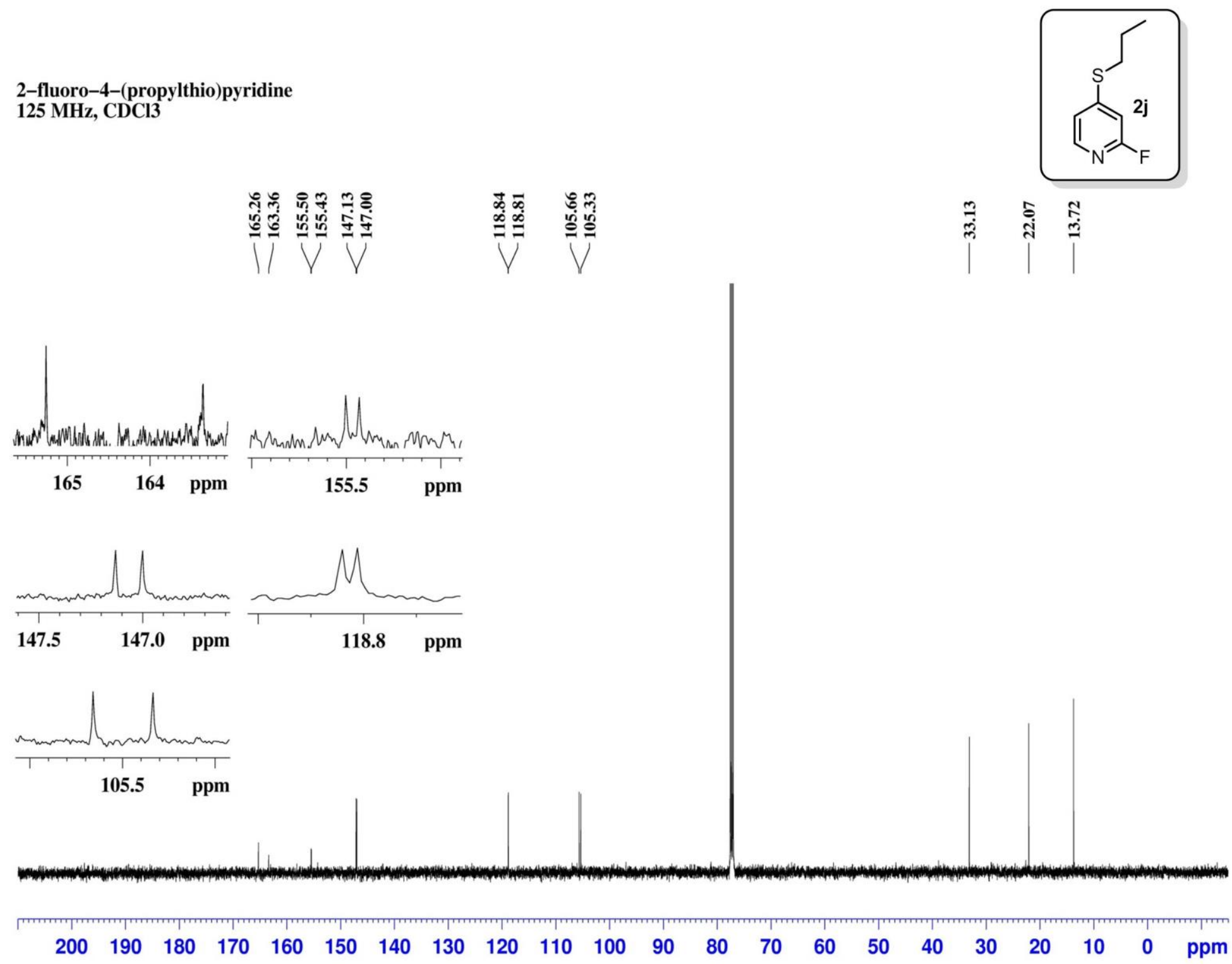
3-chloro-5-(propylthio)pyridine $125 \mathrm{MHz}, \mathrm{CDCl} 3$
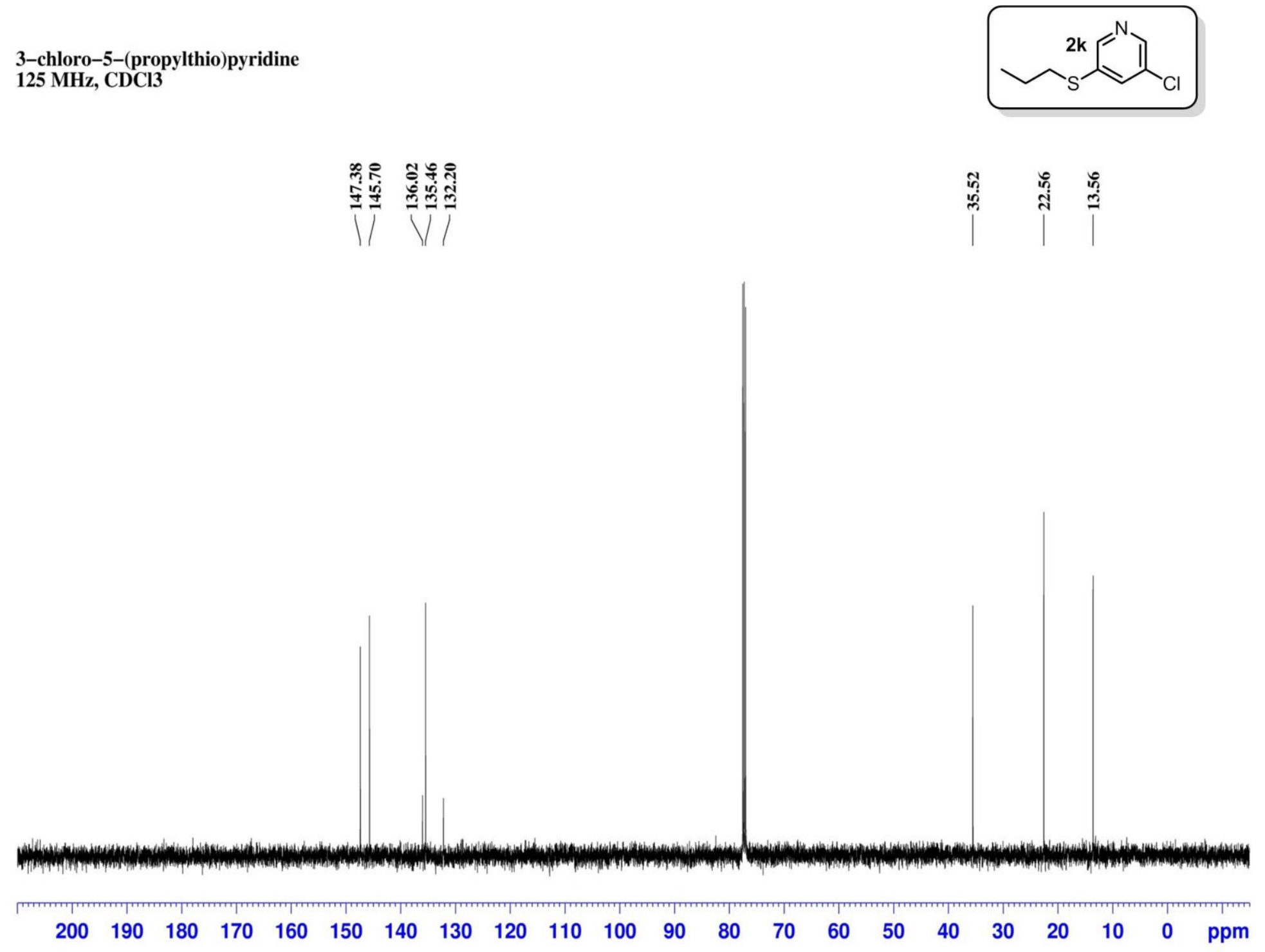
3-methoxy-5-(propylthio)pyridine

$125 \mathrm{MHz}, \mathrm{CDCl} 3$

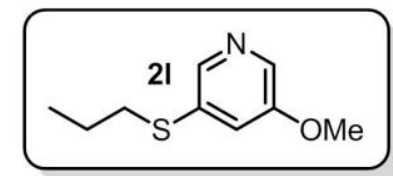

|

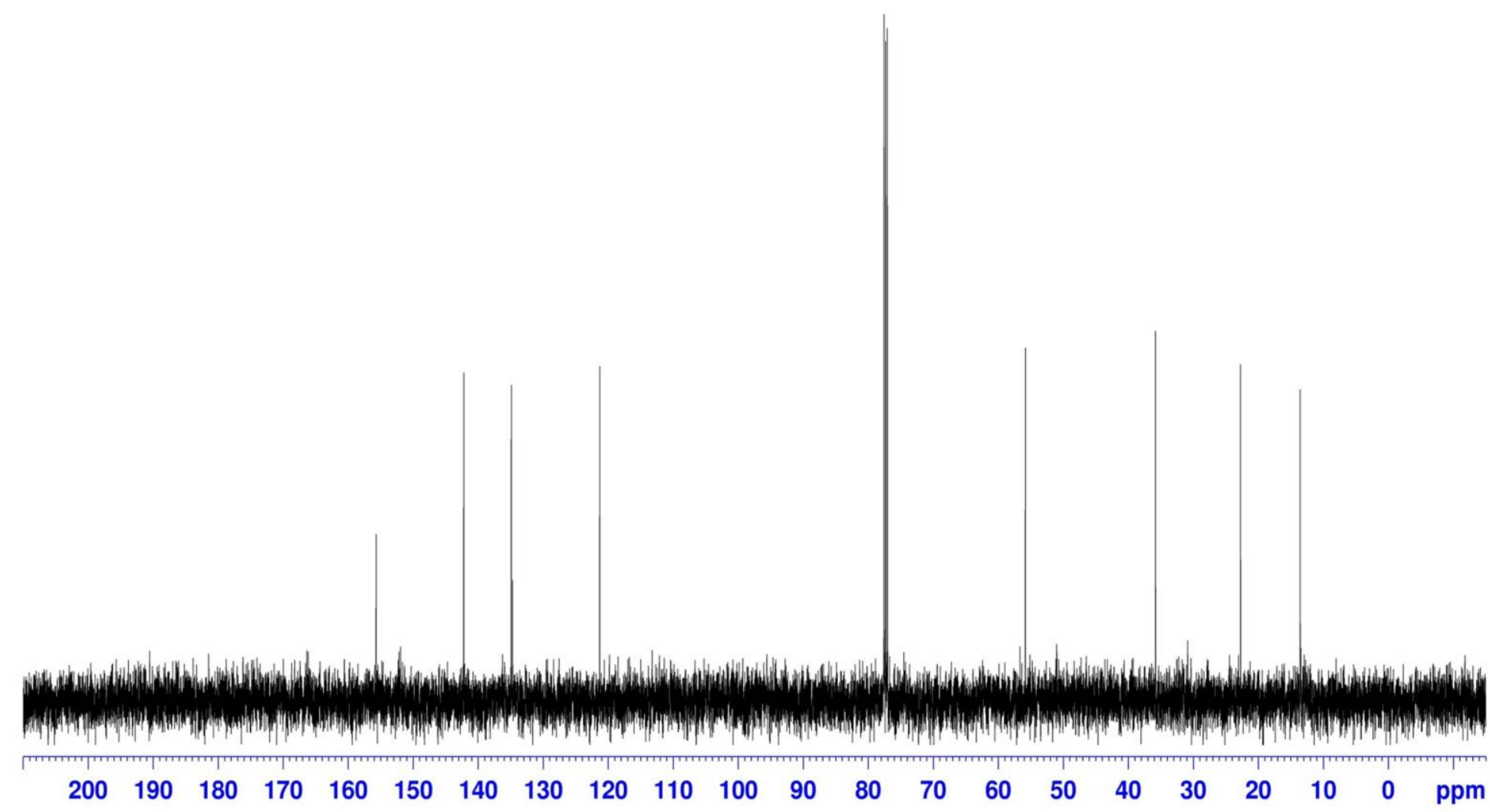




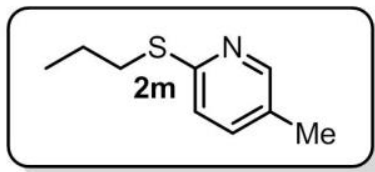

$\left.\left.\right|^{\mid}\right|^{\infty}$

్ㅏㅁ

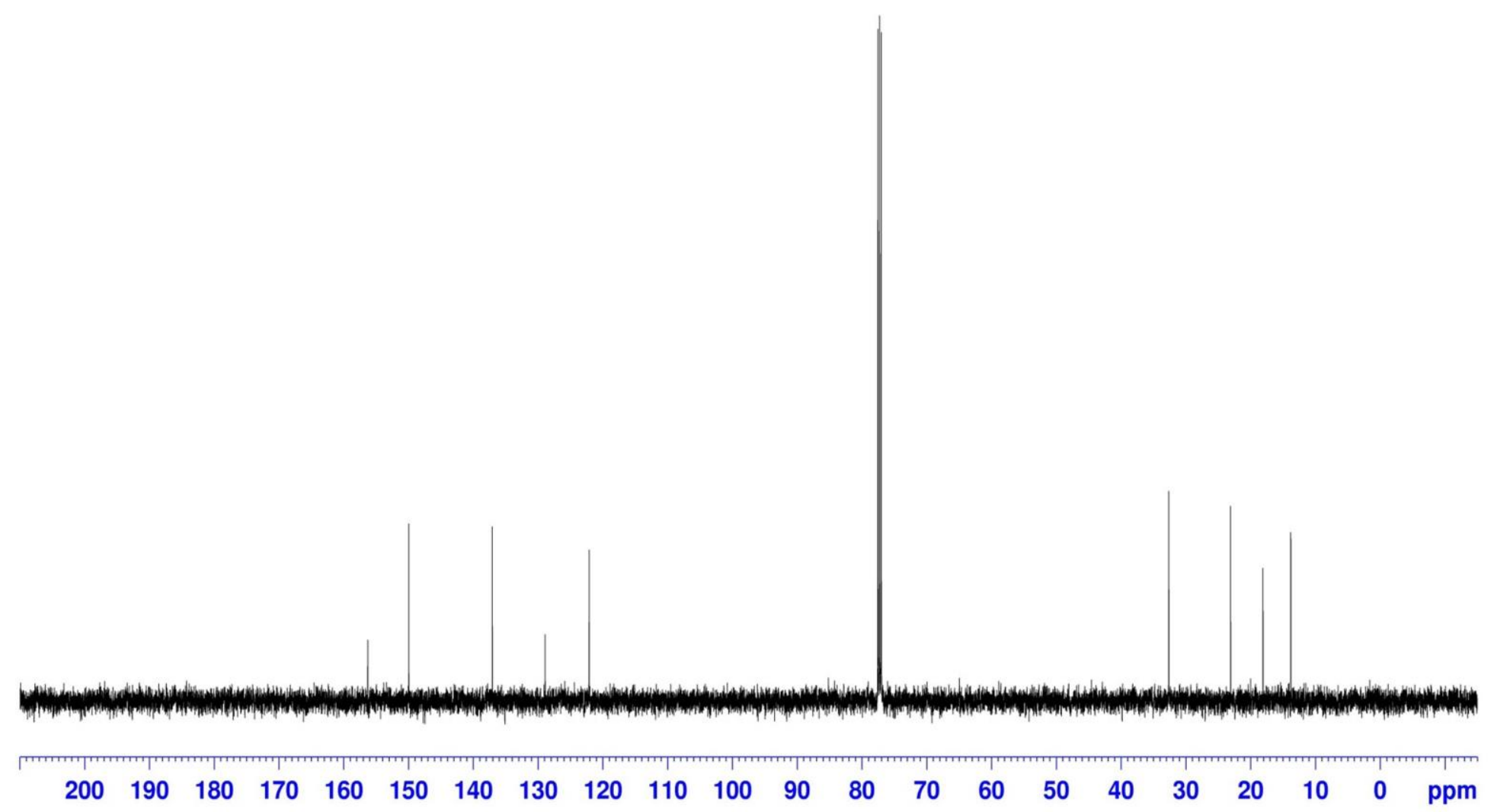


4-(propylthio)quinoline

$125 \mathrm{MHz}, \mathrm{CDCl} 3$

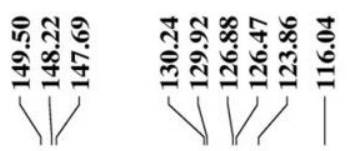

ले के ڤँ

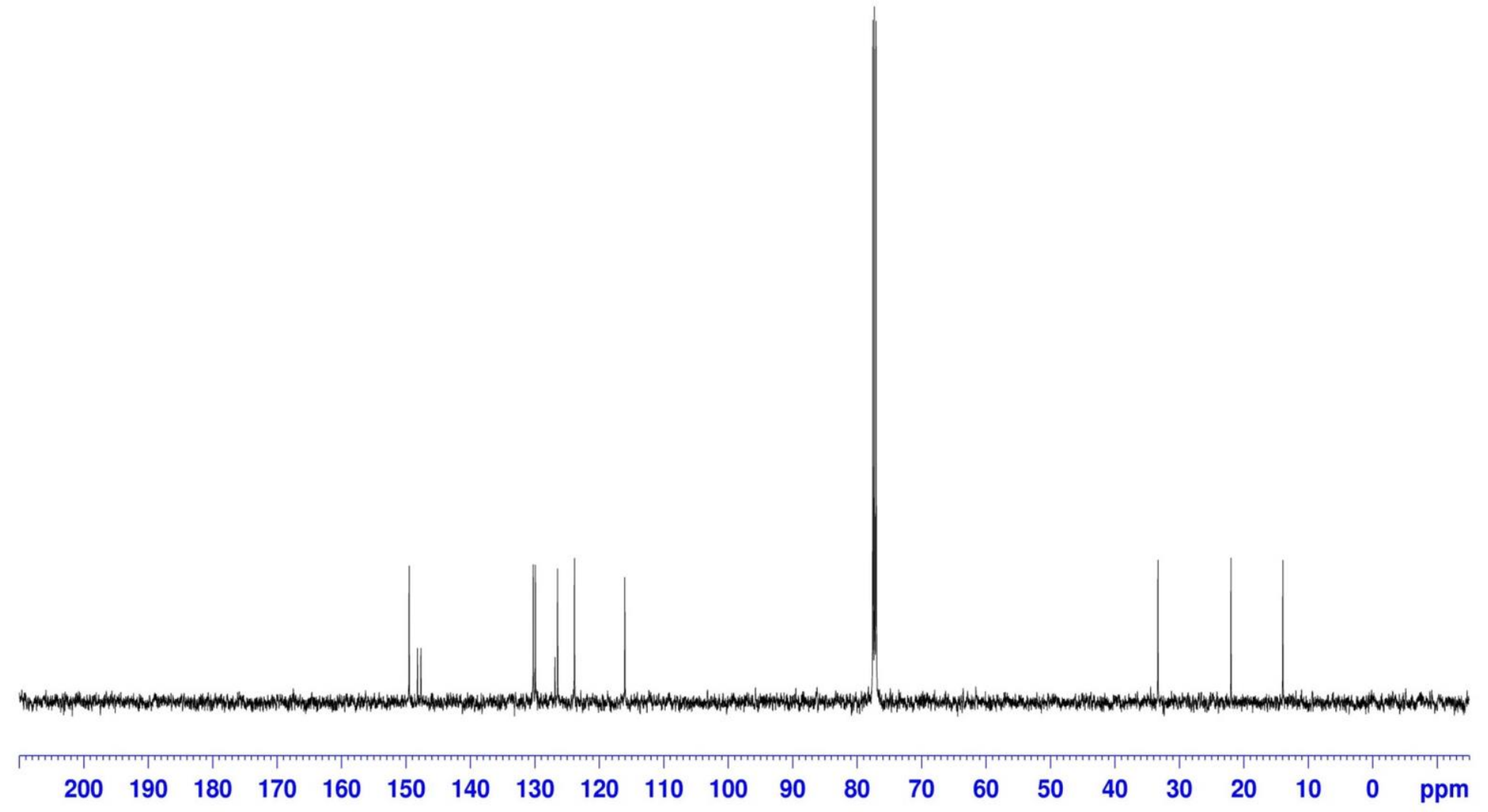


4-(propylthio)isoquinoline

$125 \mathrm{MHz}, \mathrm{CDCl} 3$
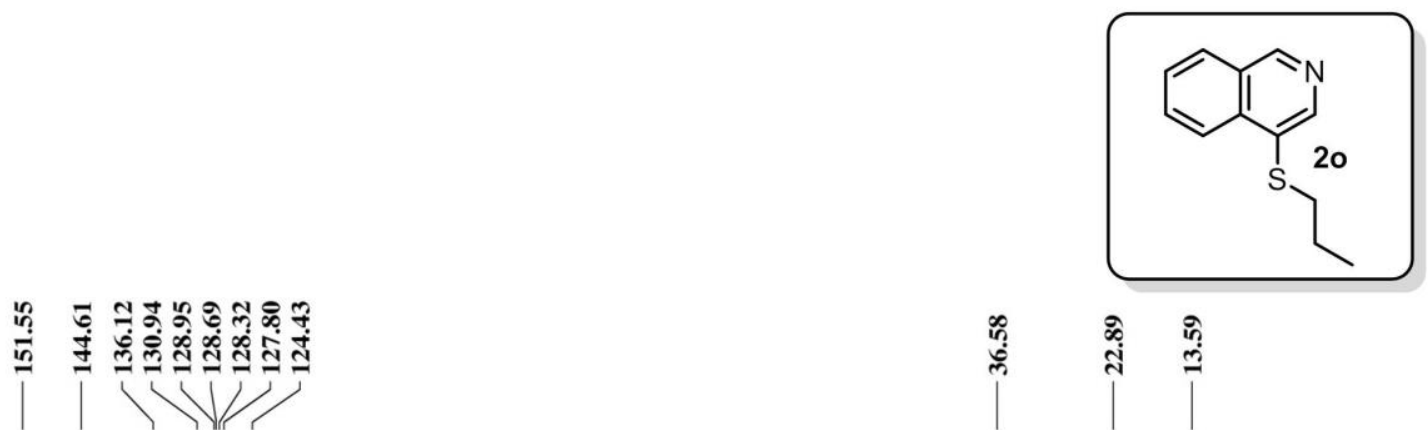

|

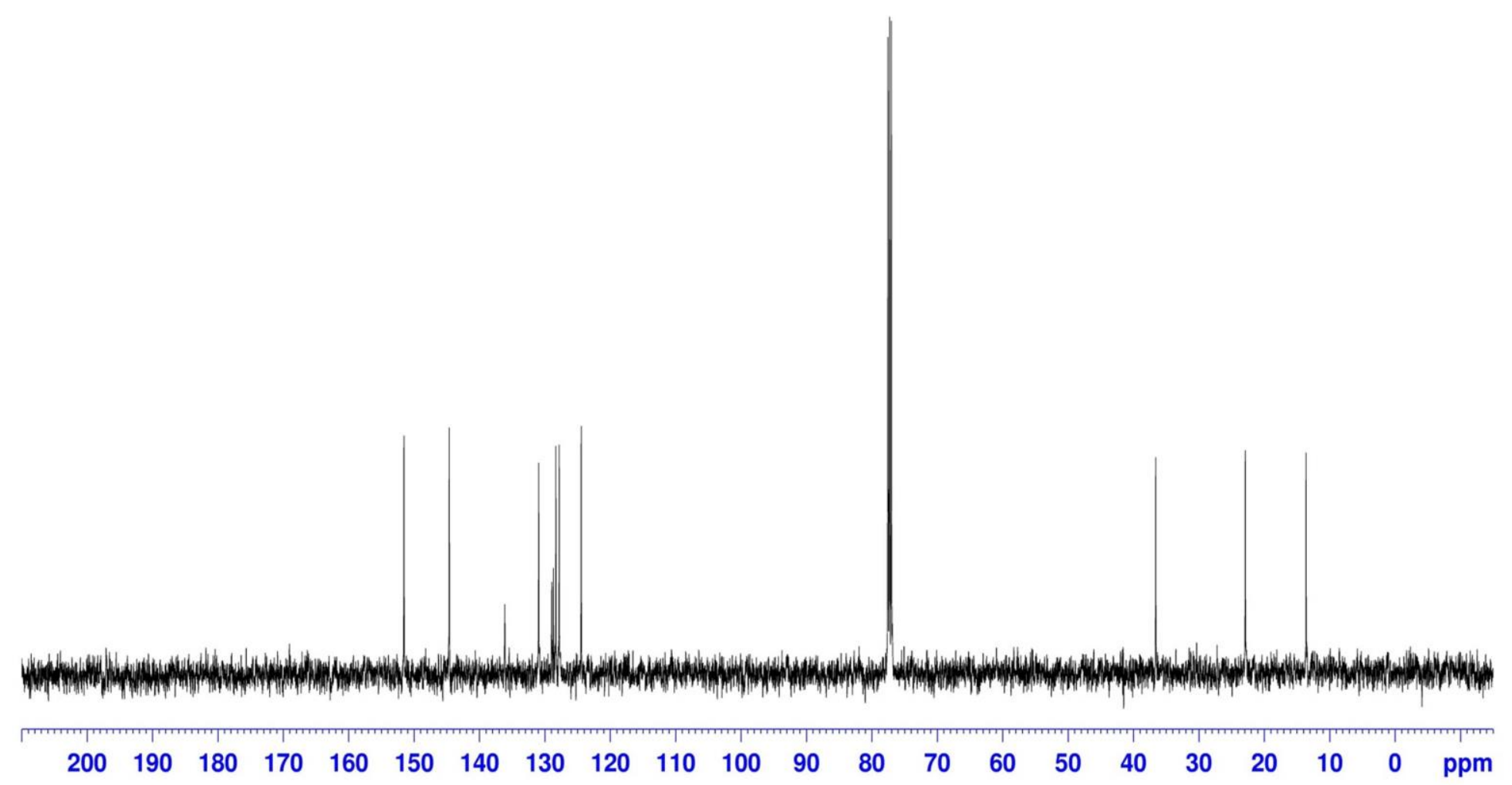


8-(propylthio)isoquinoline

$125 \mathrm{MHz}, \mathrm{CDCl} 3$
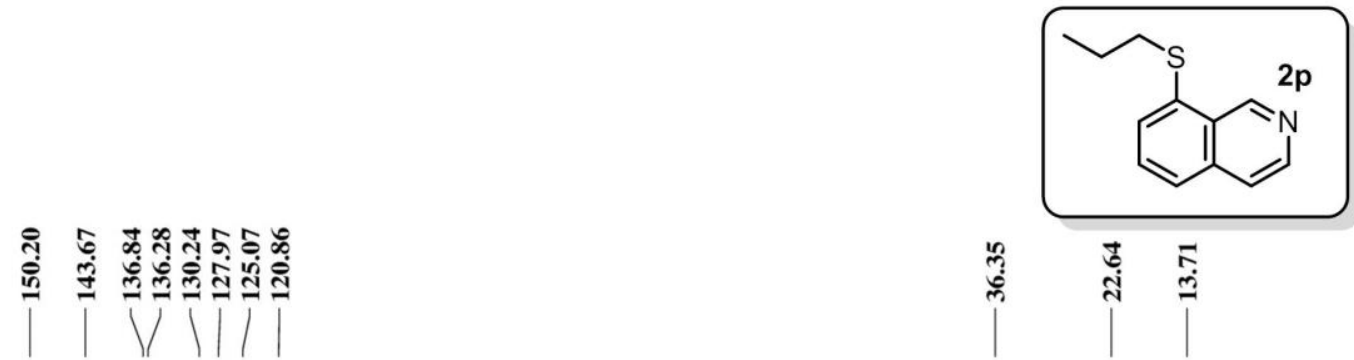

लुँ तु

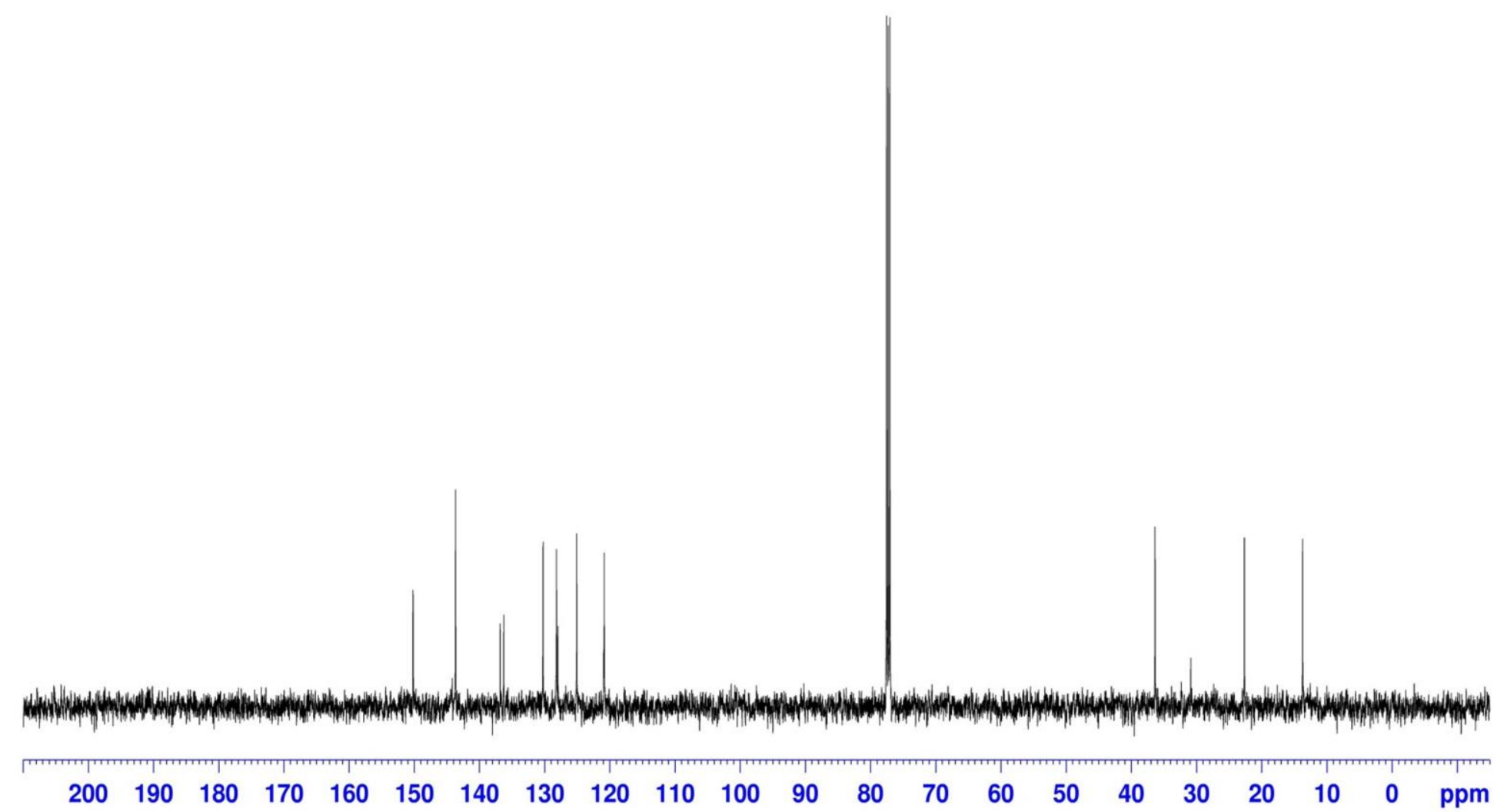


tert-butyl 5-(propylthio)-1H-indazole-1-carboxylate $125 \mathrm{MHz}, \mathrm{CDCl} 3$
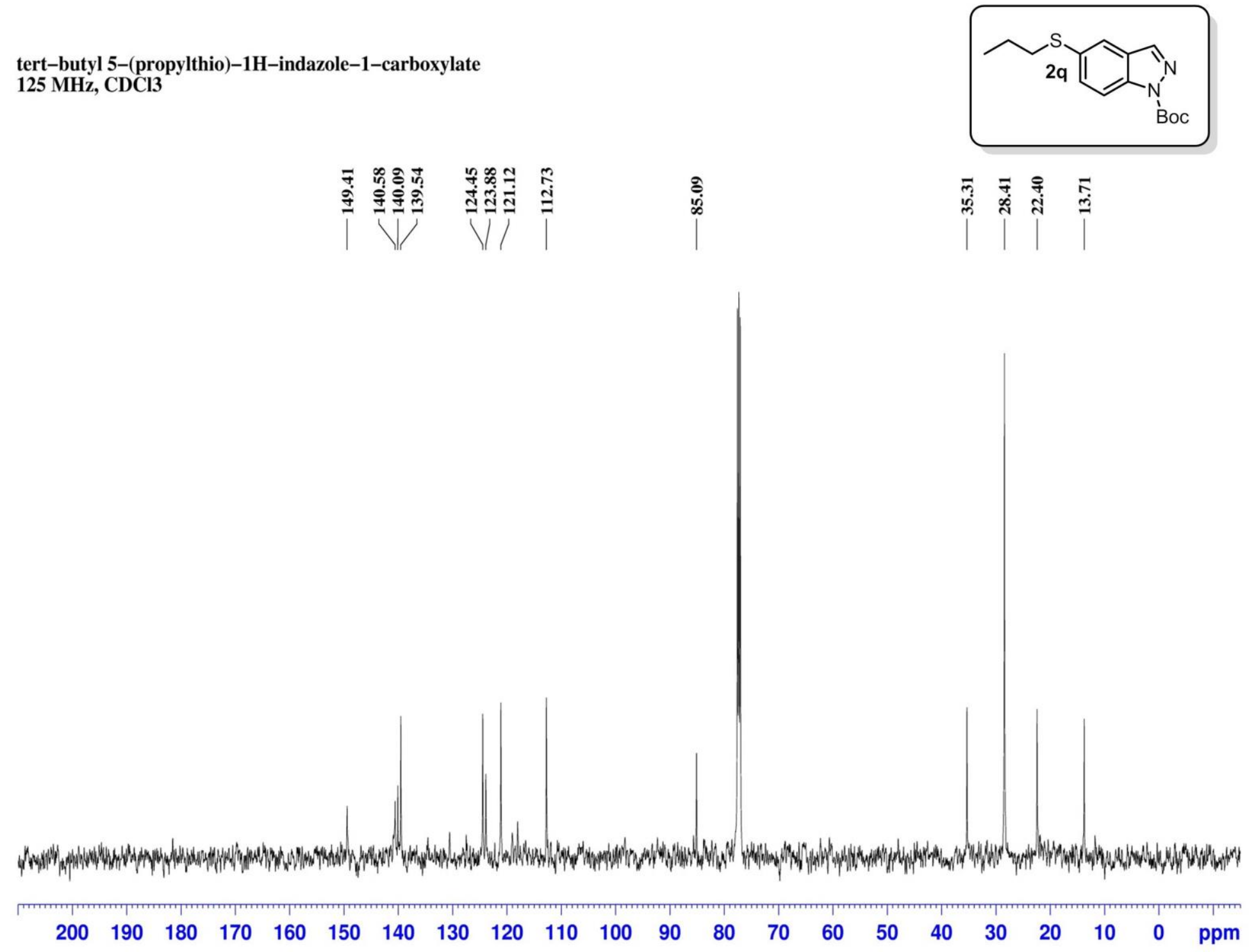
1,3,7-trimethyl-8-(propylthio)-1H-purine-2,6(3H,7H)-dione $125 \mathrm{MHz}, \mathrm{CDCl} 3$
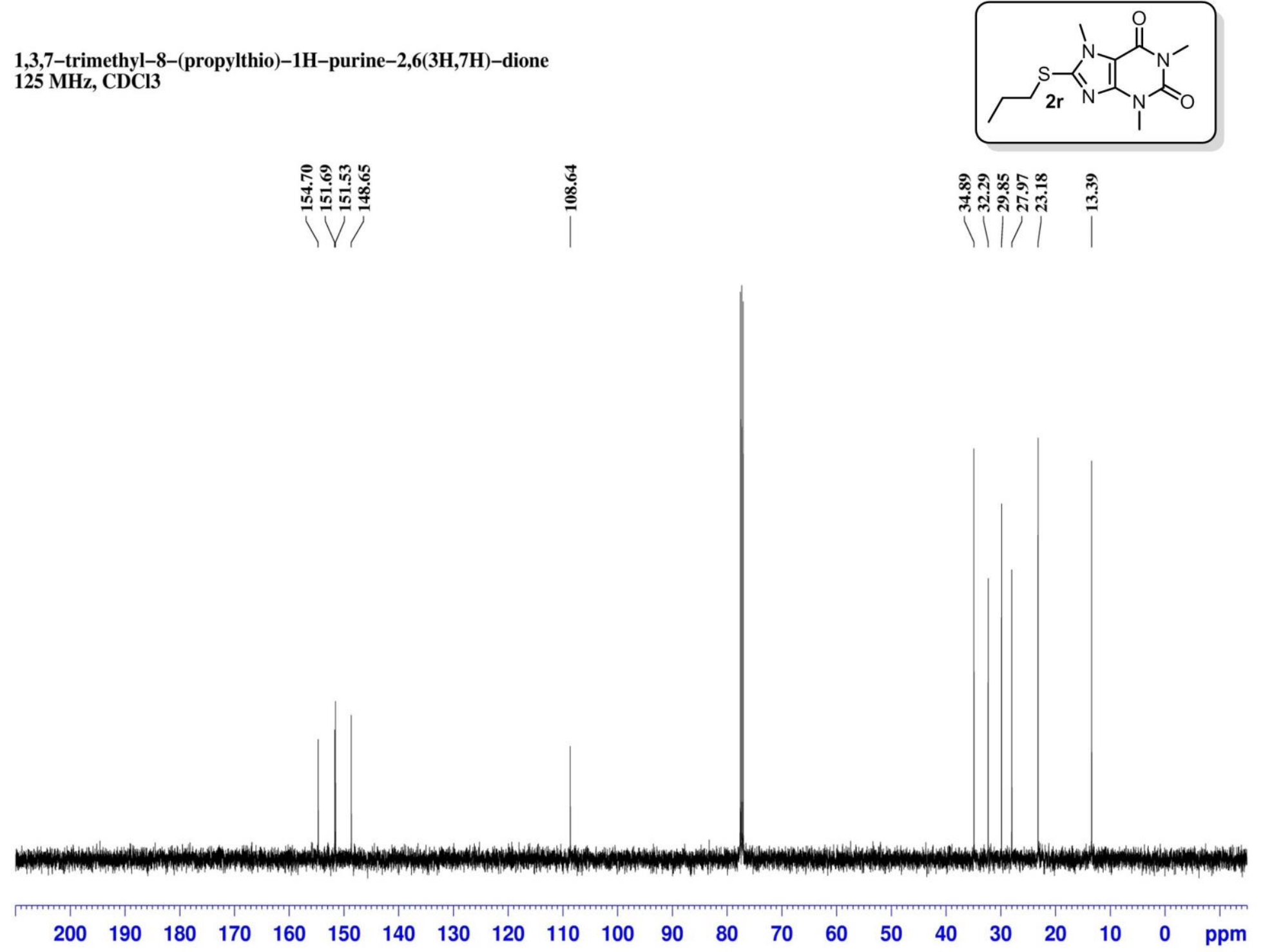
4-(butylthio)benzonitrile

$125 \mathrm{MHz}, \mathrm{CDCl} 3$

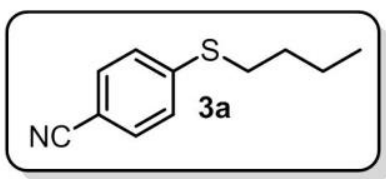

|

लंল

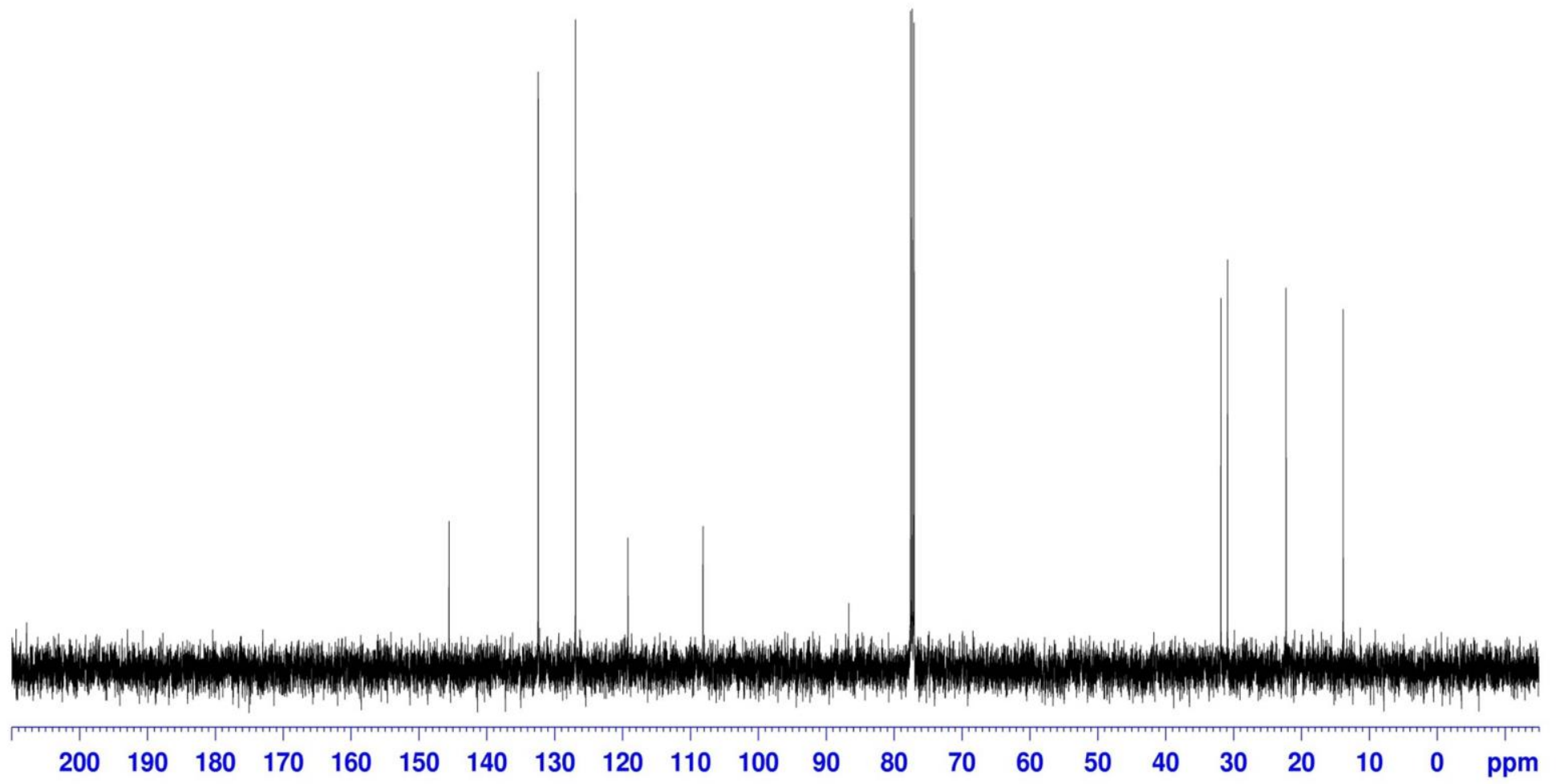



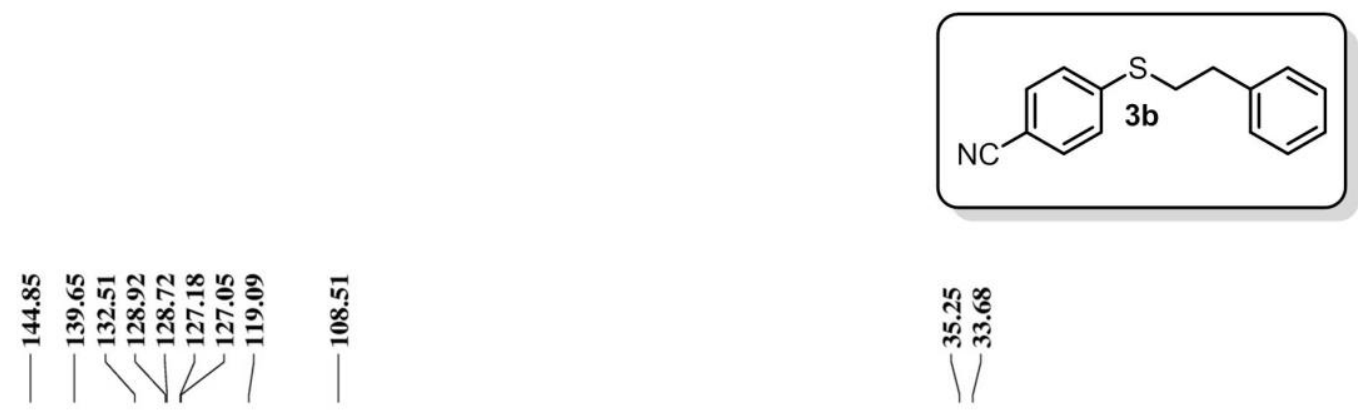

ฝึ่

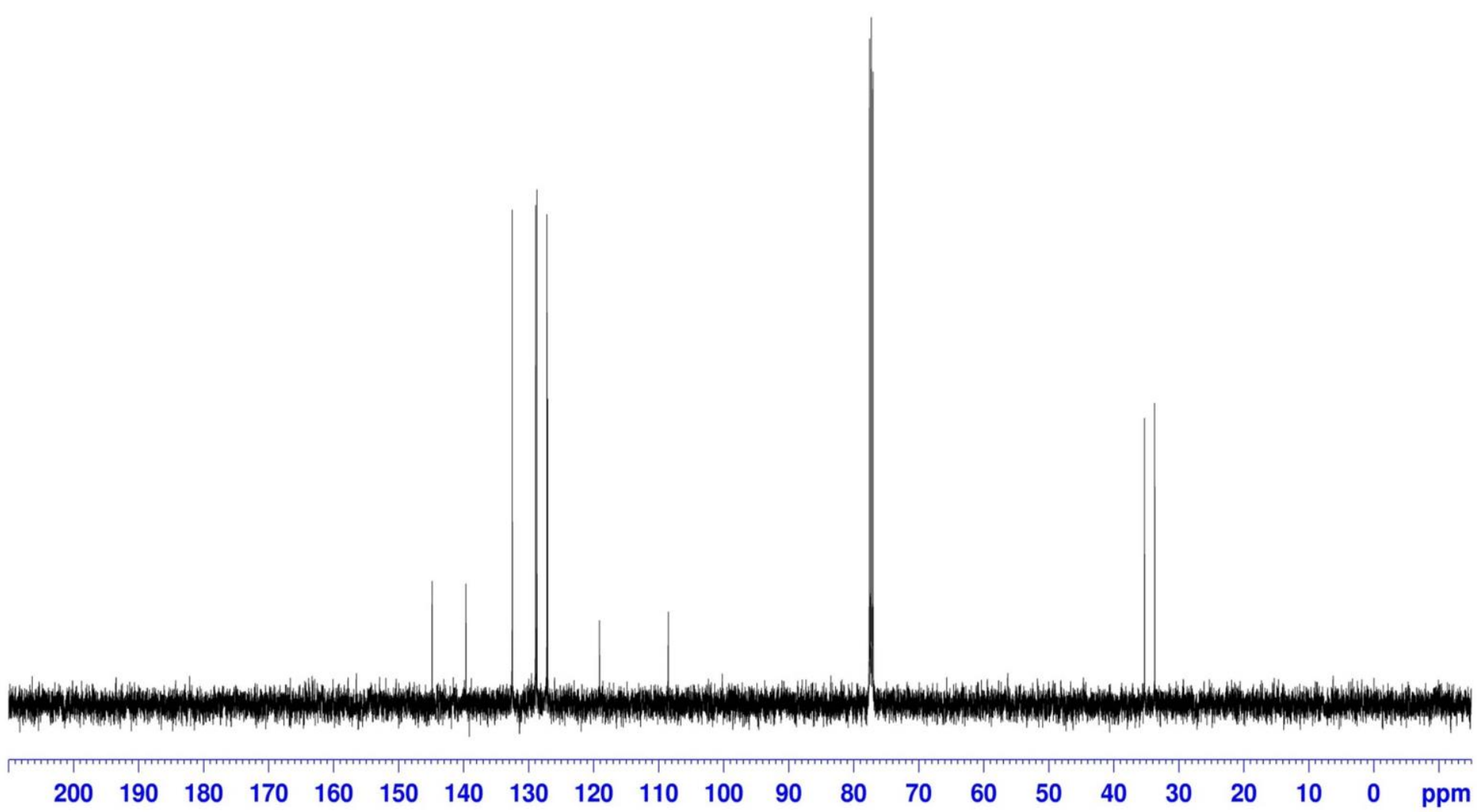


methyl 3-((4-cyanophenyl)thio)propanoate $125 \mathrm{MHz}, \mathrm{CDCl} 3$
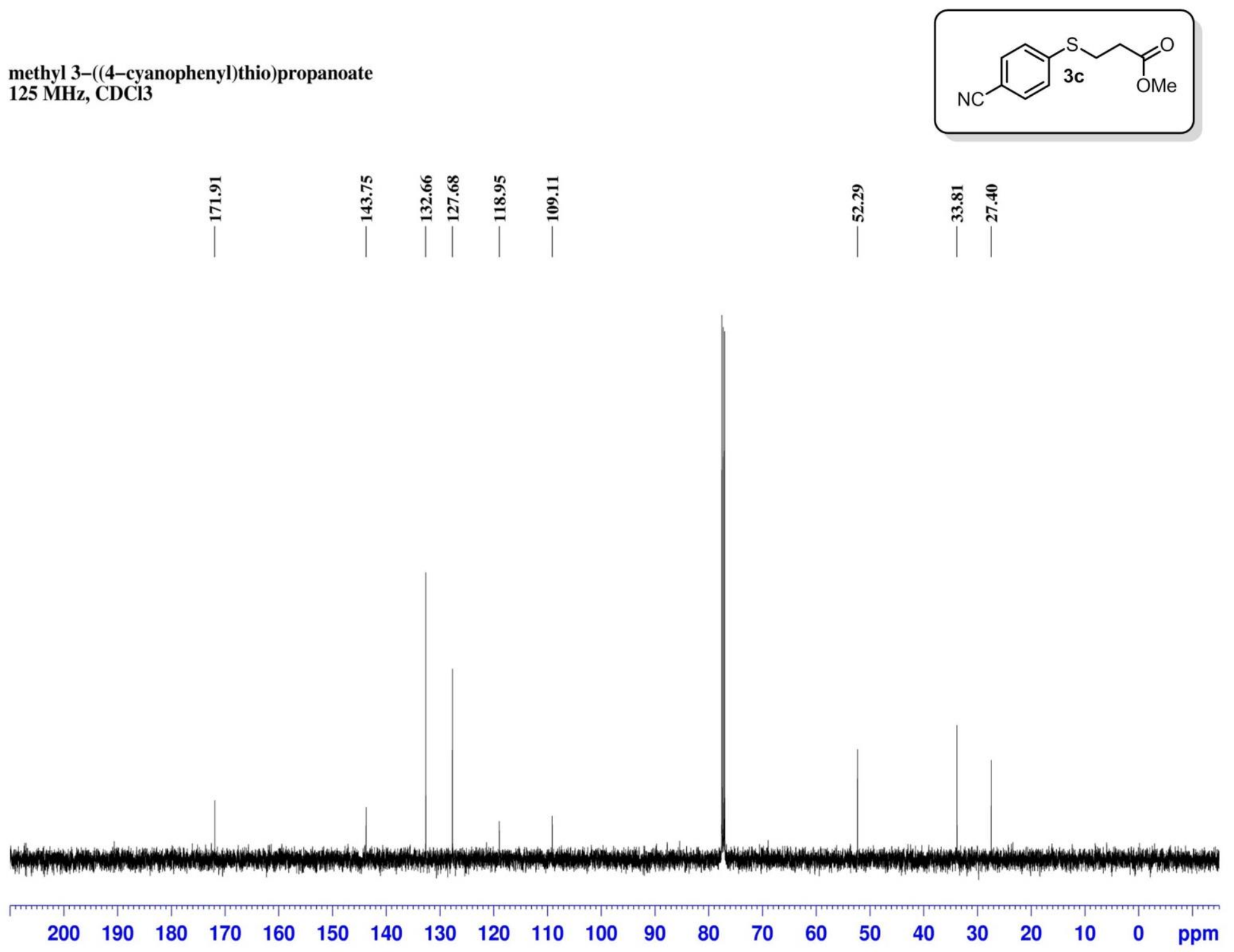
4-((2-hydroxyethyl)thio)benzonitrile $125 \mathrm{MHz}, \mathrm{CDCl} 3$
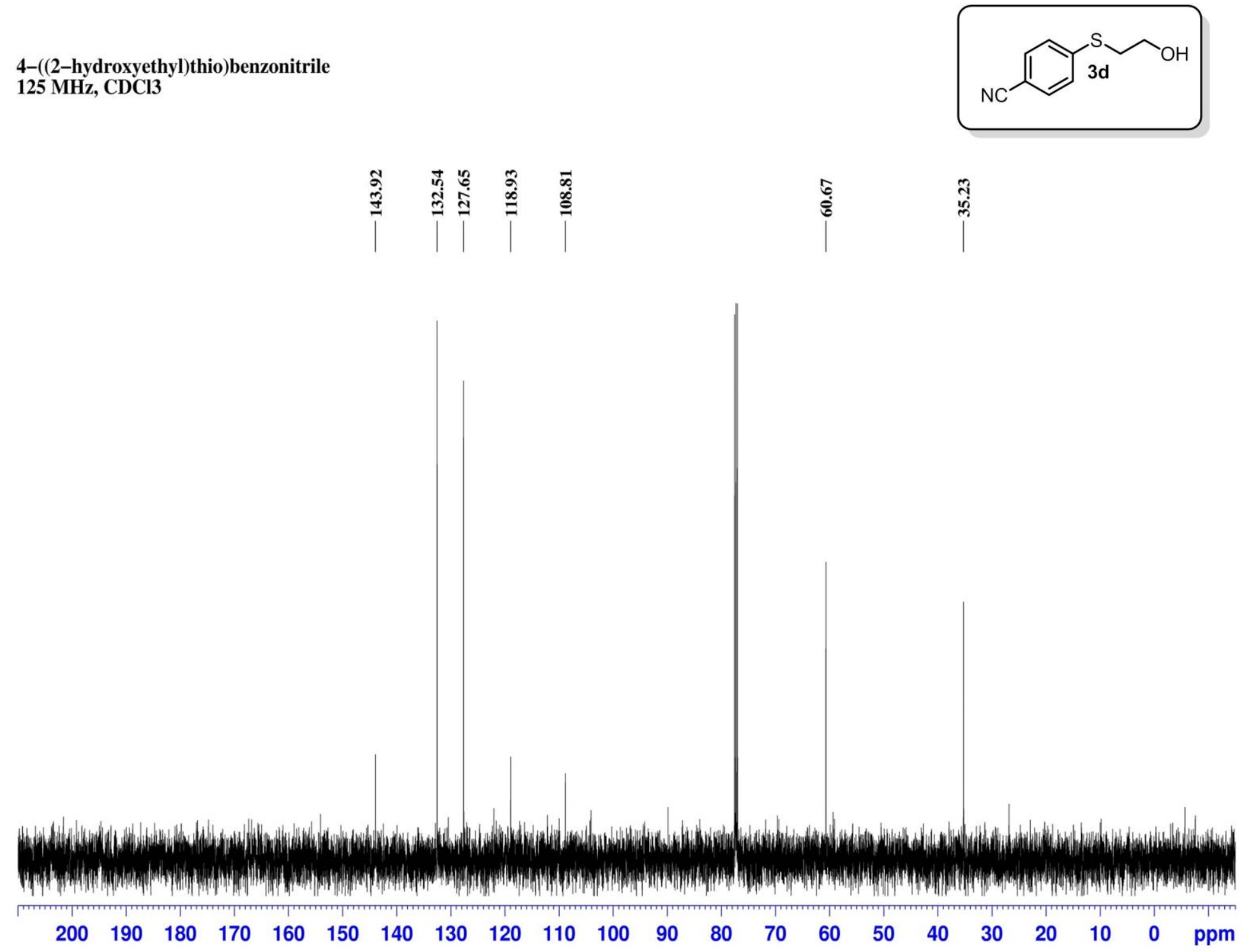
4-((2-aminoethyl)thio)benzonitrile $125 \mathrm{MHz}, \mathrm{CDCl} 3$
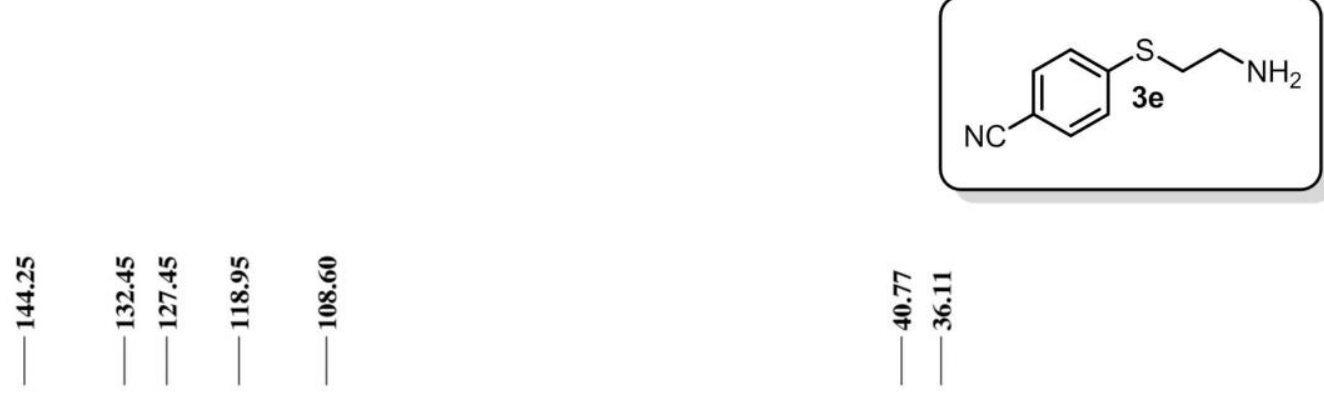

응

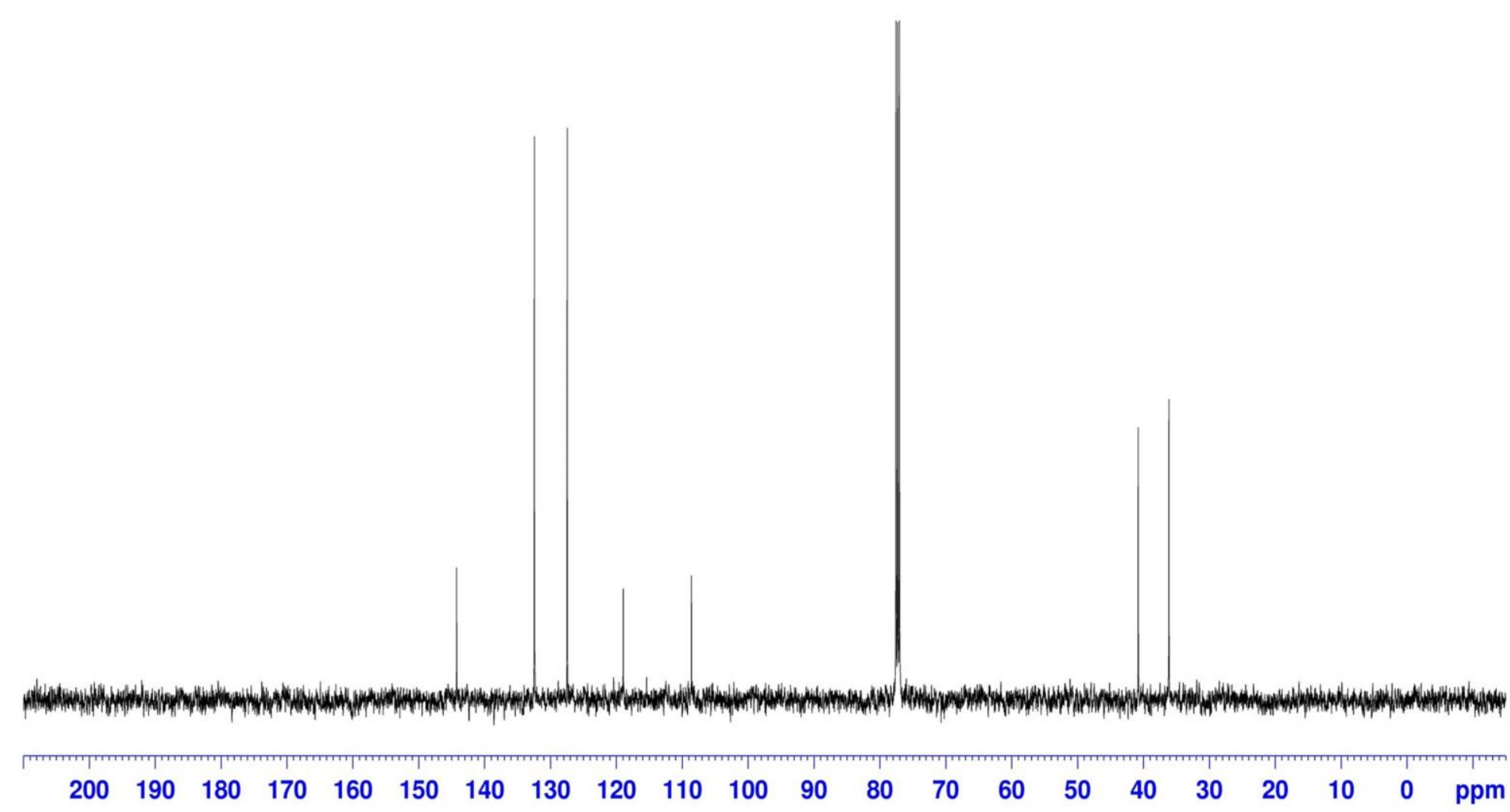


4-(cyclohexylthio)benzonitrile

$125 \mathrm{MHz}, \mathrm{CDCl} 3$
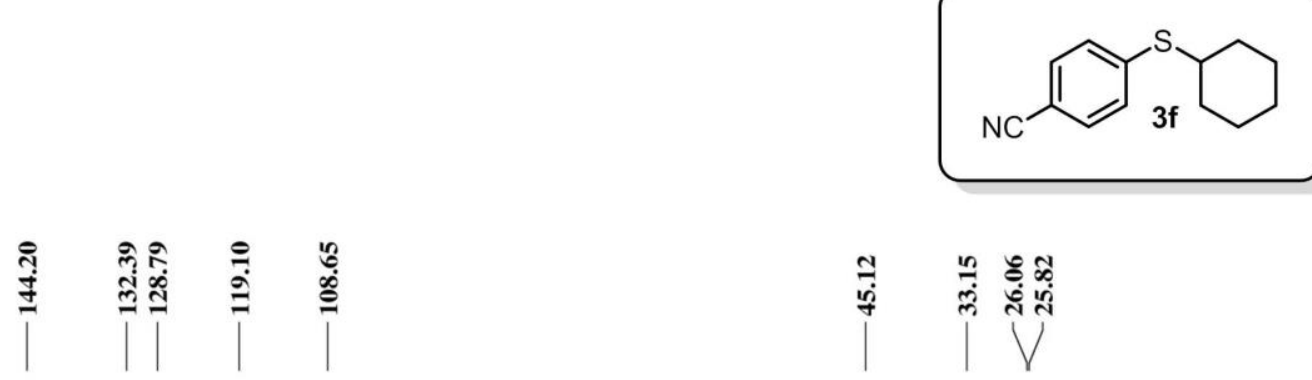

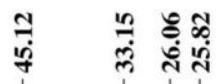

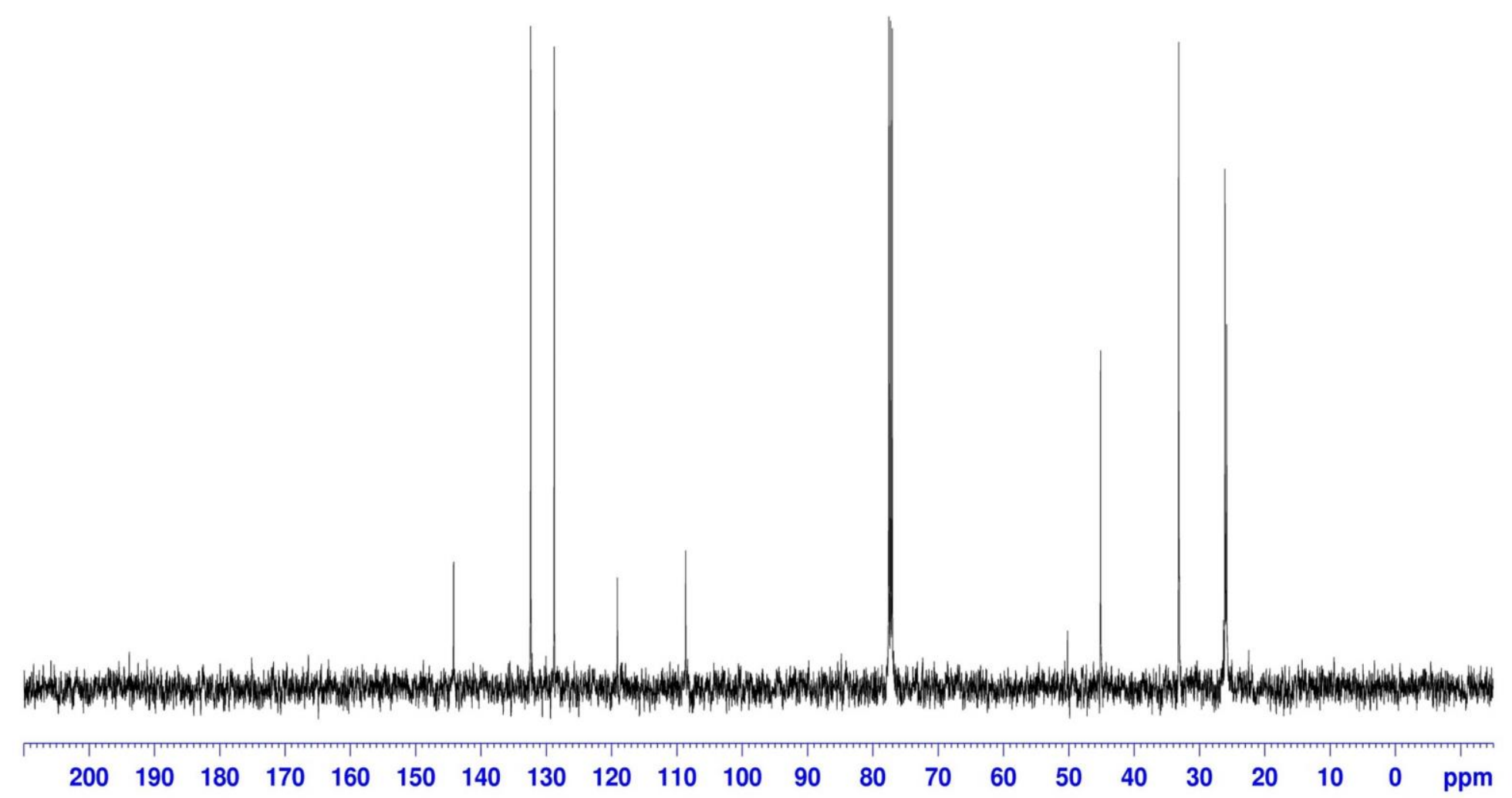


4-((1-hydroxyhexan-3-yl)thio)benzonitrile $125 \mathrm{MHz}, \mathrm{CDCl} 3$
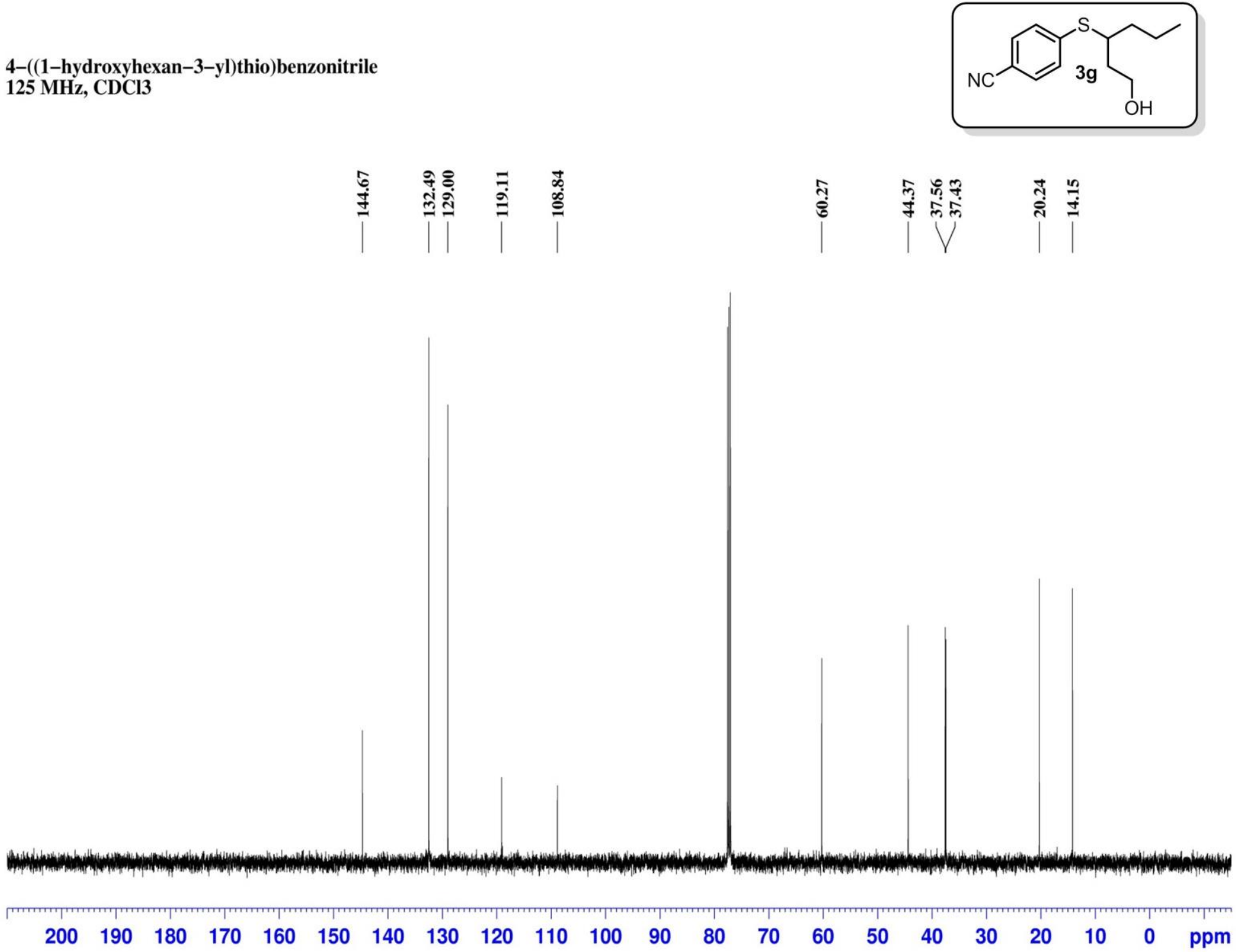
(R)-methyl 2-((tert-butoxycarbonyl)amino)-3-((4-cyanophenyl)thio)propanoate $125 \mathrm{MHz}, \mathrm{CDCl} 3$

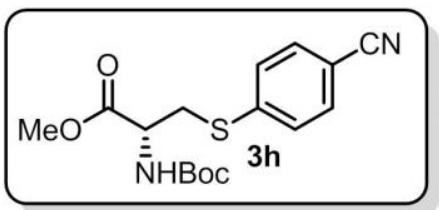

$$
\text { }
$$

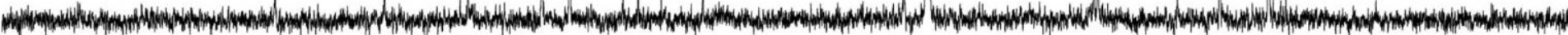

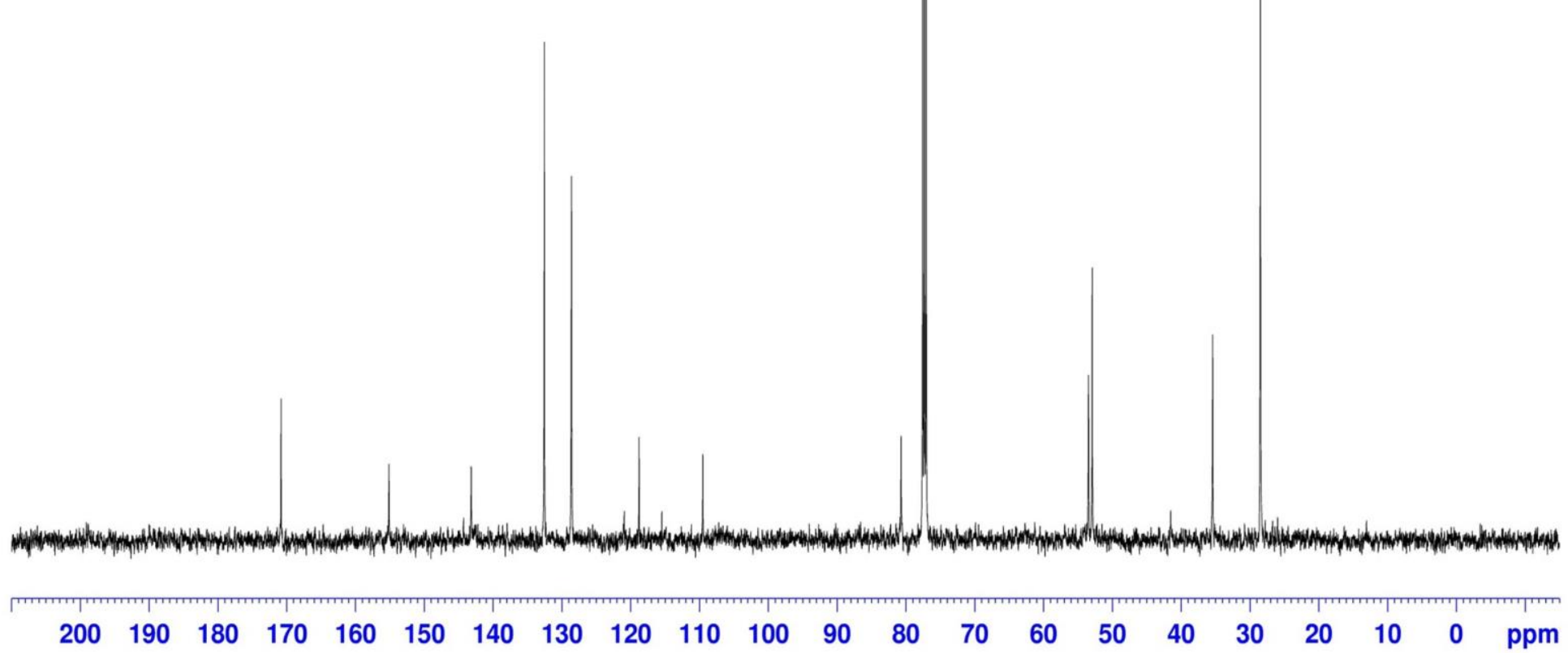


(4-(tert-butylthio)benzonitrile

$125 \mathrm{MHz}, \mathrm{CDCl} 3$

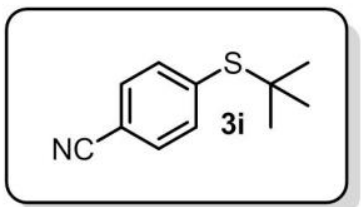

$\mid$

$\stackrel{\text { गे }}{\stackrel{5}{5}}$

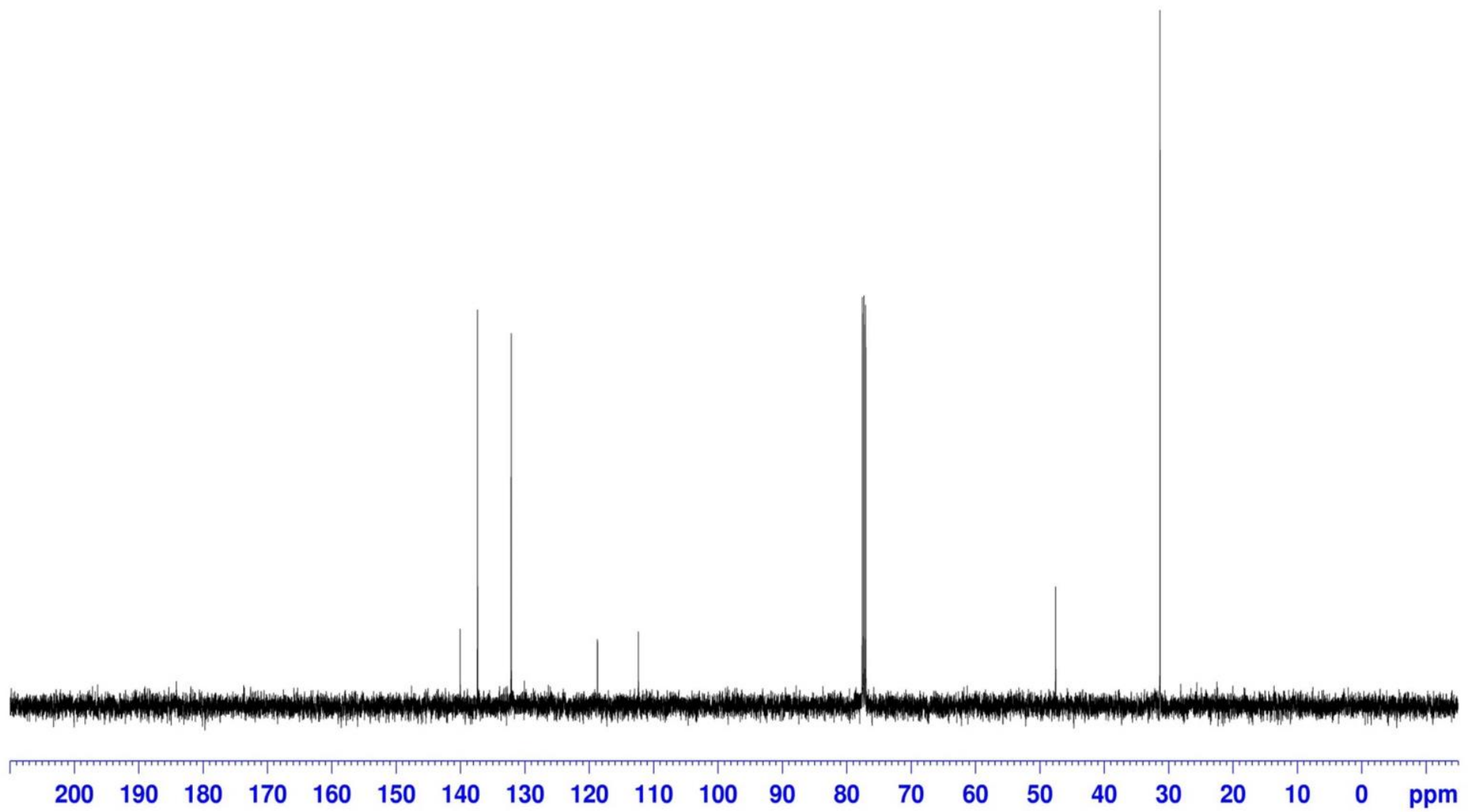


4-(adamantan-1-ylthio)benzonitrile 125 MHz, CDCl3
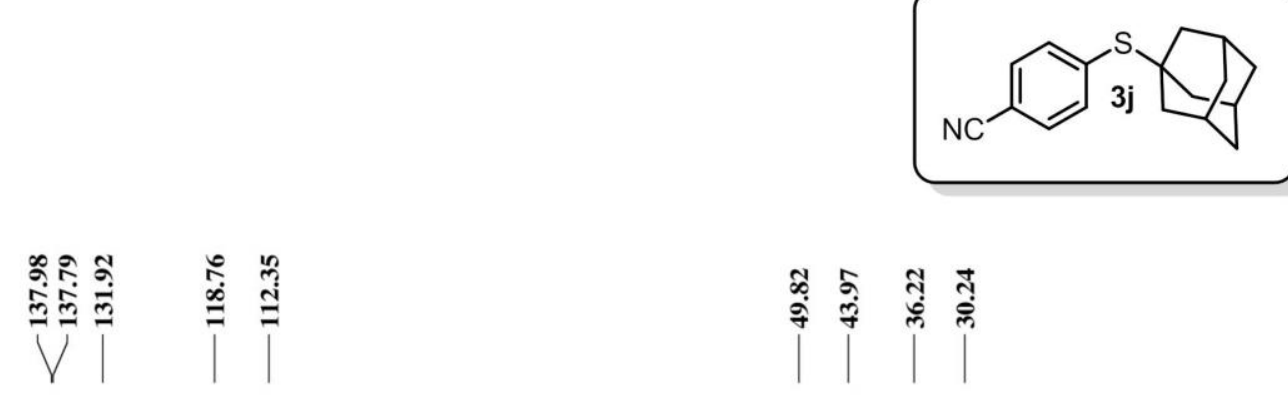

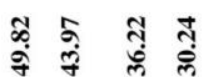

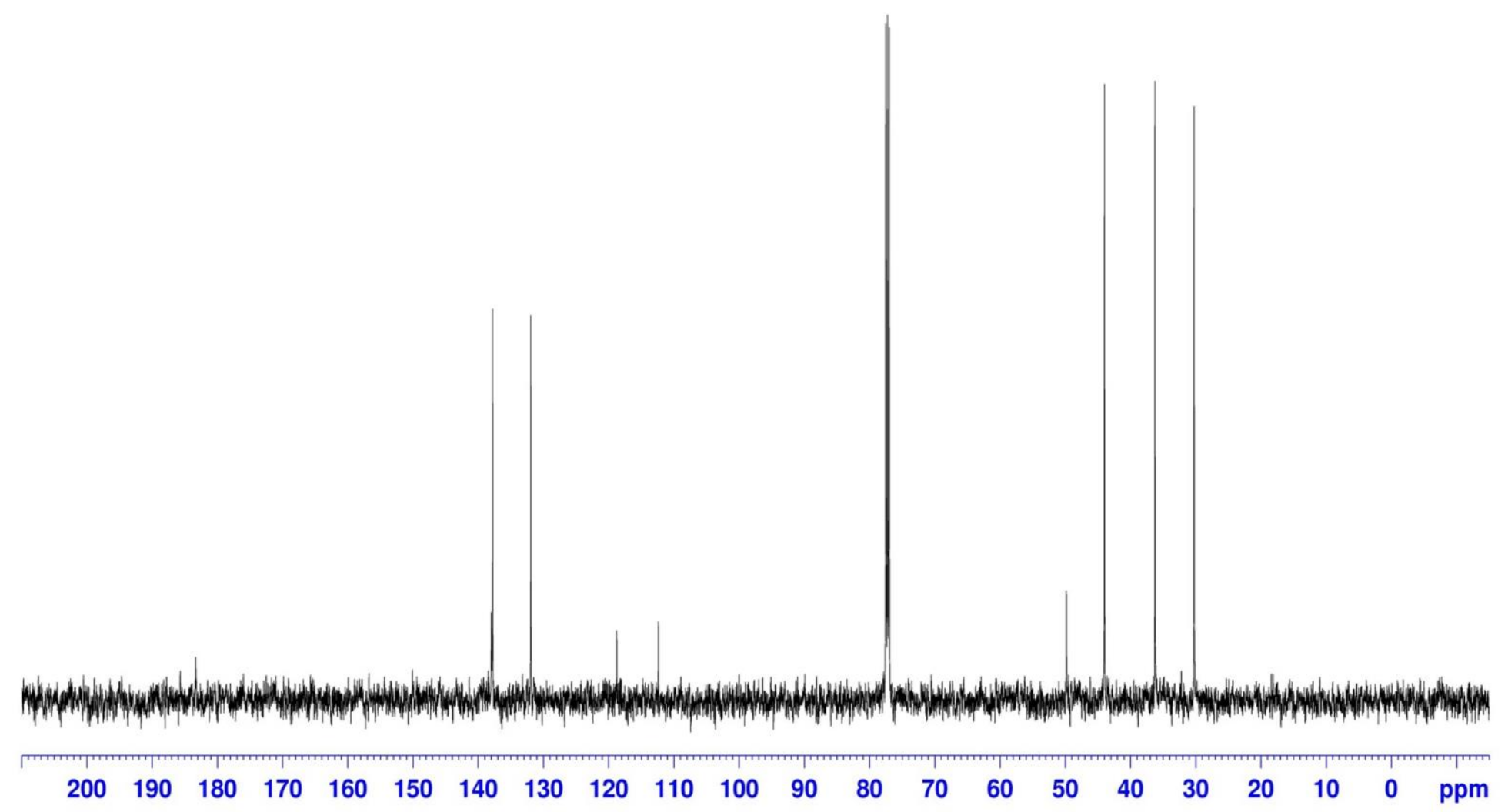


(1S,4S)- \& (1R,4R)-4-((2-(4-Methyl-2-oxocyclohexyl)propan-2-yl)thio)benzonitrile $125 \mathrm{MHz}, \mathrm{CDCl} 3$
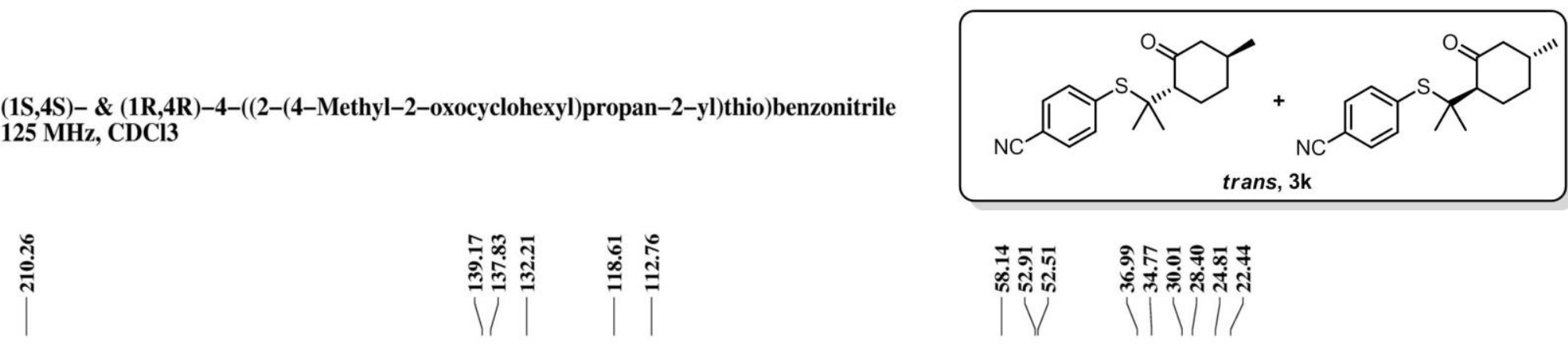

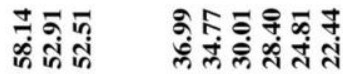

IV l| ||l|

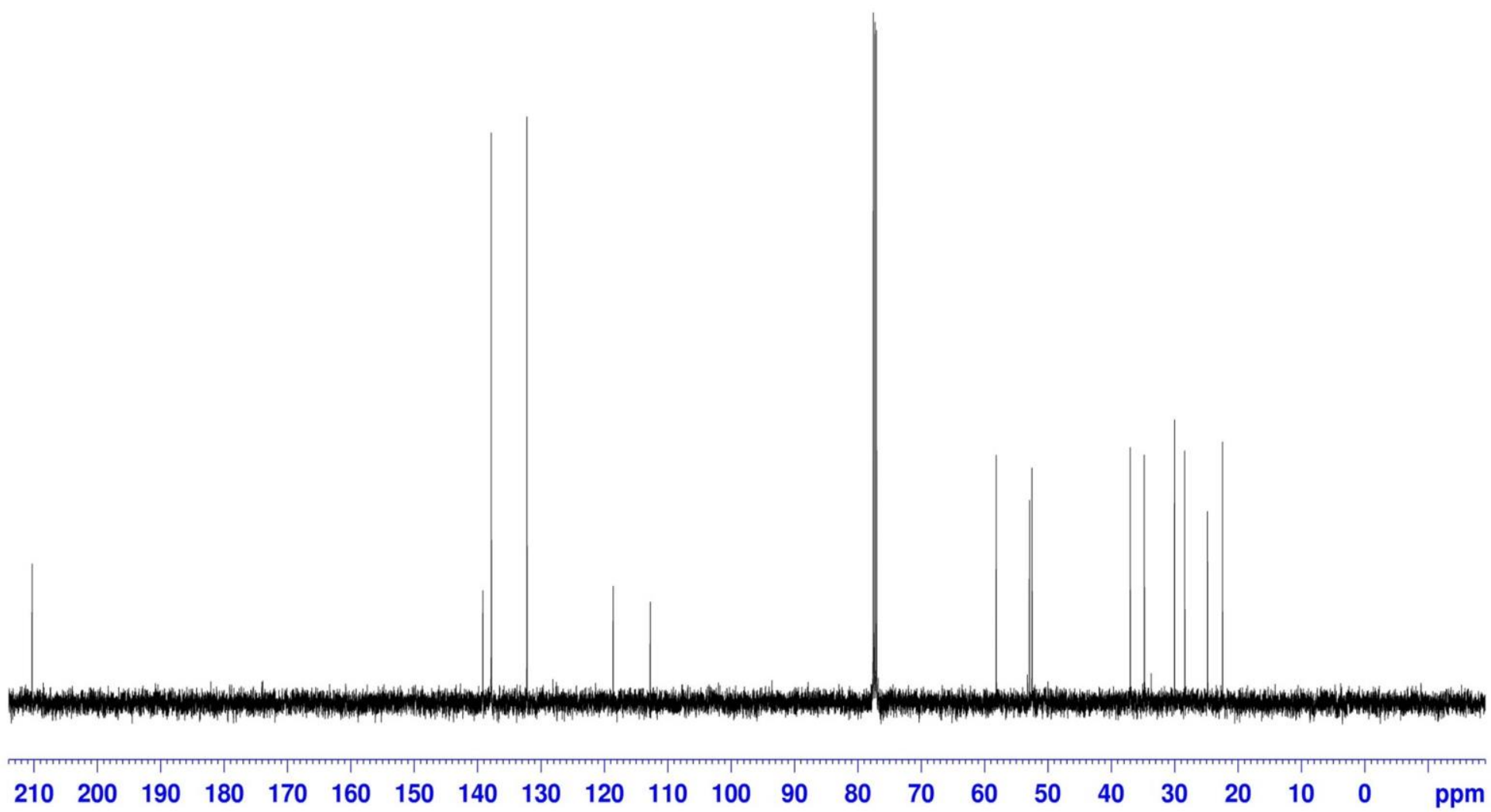


(1R,4S)- \& (1R,4S)-4-((2-(4-Methyl-2-oxocyclohexyl)propan-2-yl)thio)benzonitrile $125 \mathrm{MHz}, \mathrm{CDCl} 3$
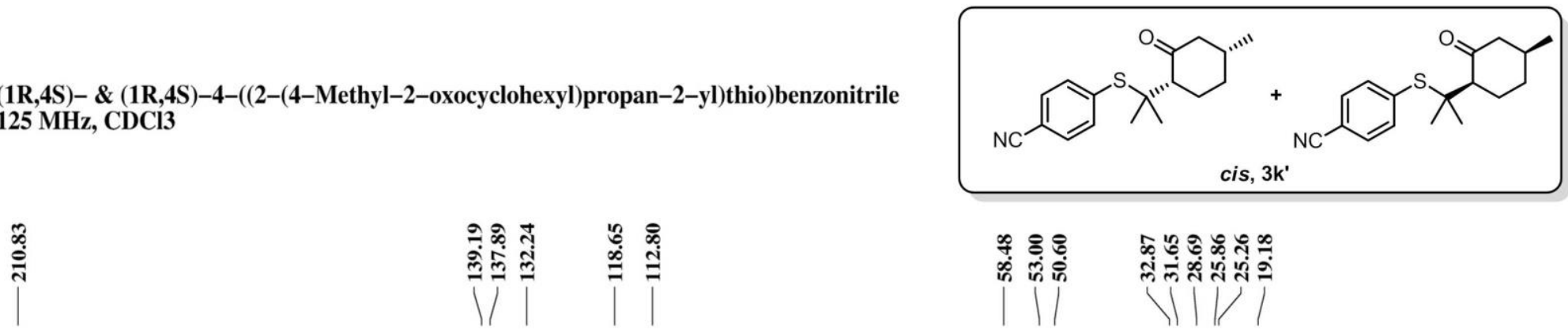

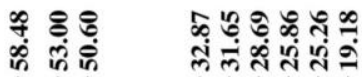

II VIII

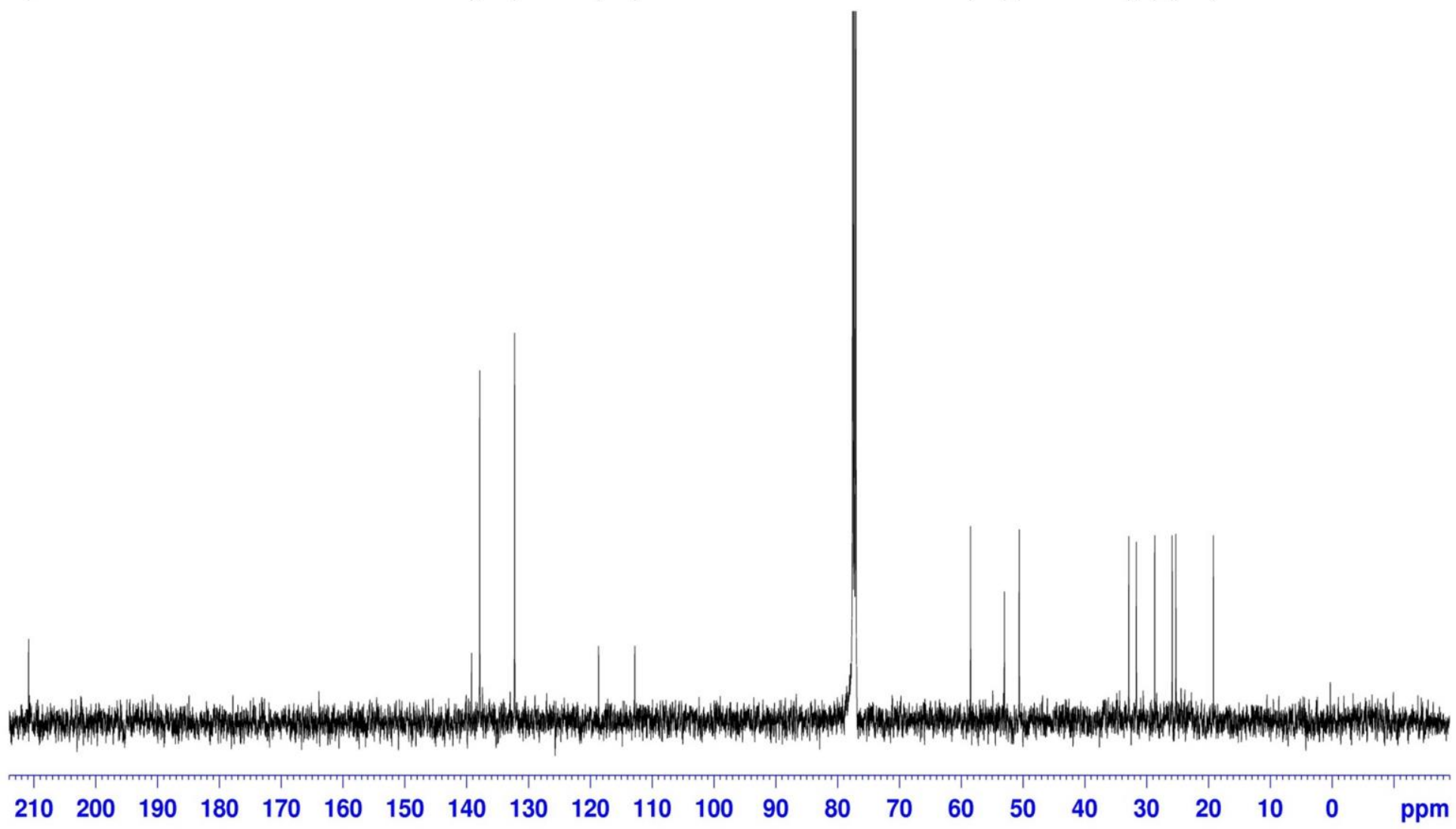


(R)-methyl 2-((tert-butoxycarbonyl)amino)-3-((2-fluoropyridin-4-yl)thio)propanoate $125 \mathrm{MHz}, \mathrm{CDCl} 3$
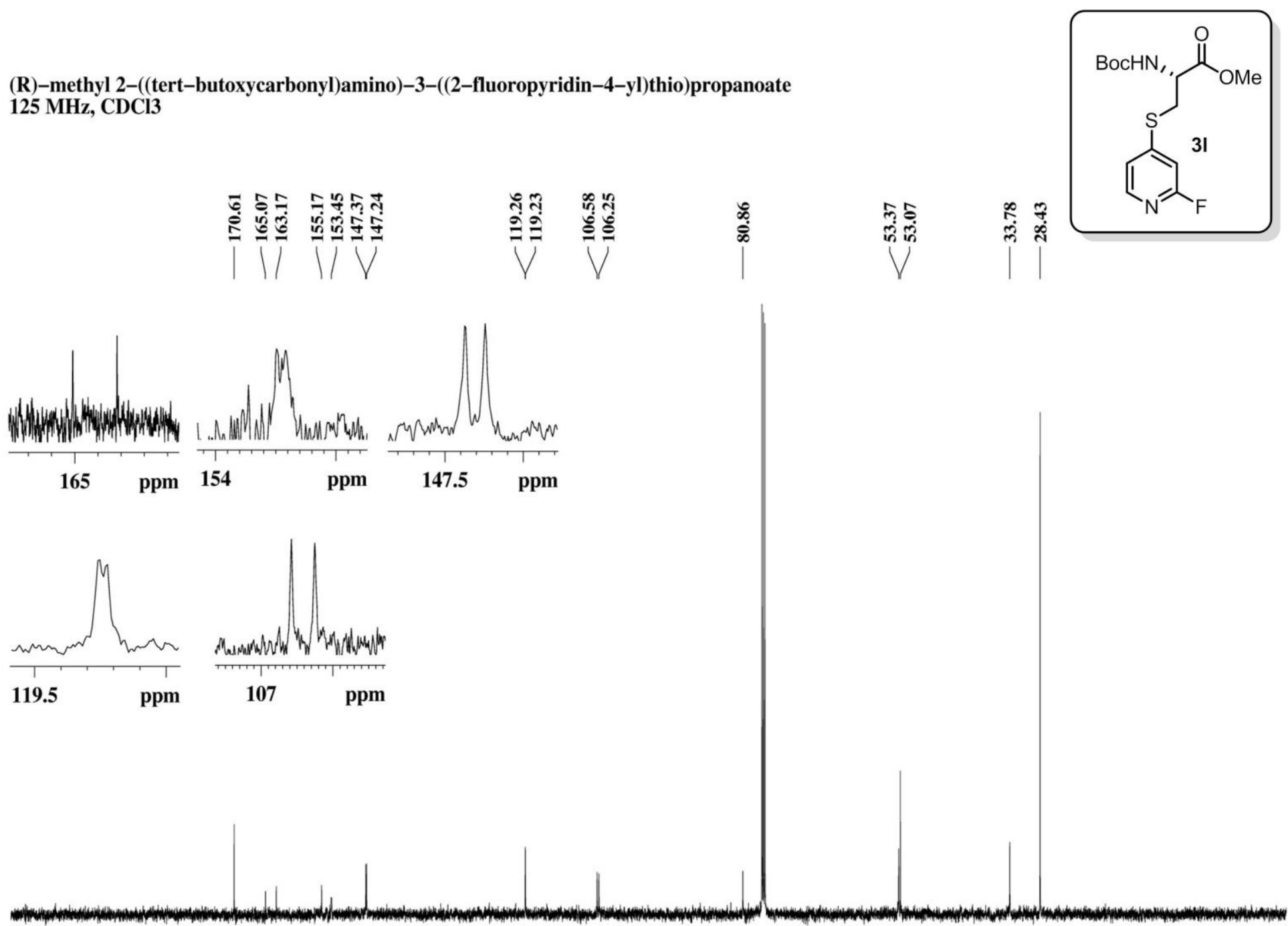

$\begin{array}{llllllllllll}200 & 190 & 180 & 170 & 160 & 150 & 140 & 130 & 120 & 110 & 100 & 90\end{array}$

$80 \quad 70 \quad 60$

$50 \quad 40$

$30 \quad 20$

$10 \quad 0 \quad \mathrm{ppm}$ 
(R)-methyl 2-((tert-butoxycarbonyl)amino)-3-((5-methoxypyridin-3-yl)thio)propanoate $125 \mathrm{MHz}, \mathrm{CDCl} 3$
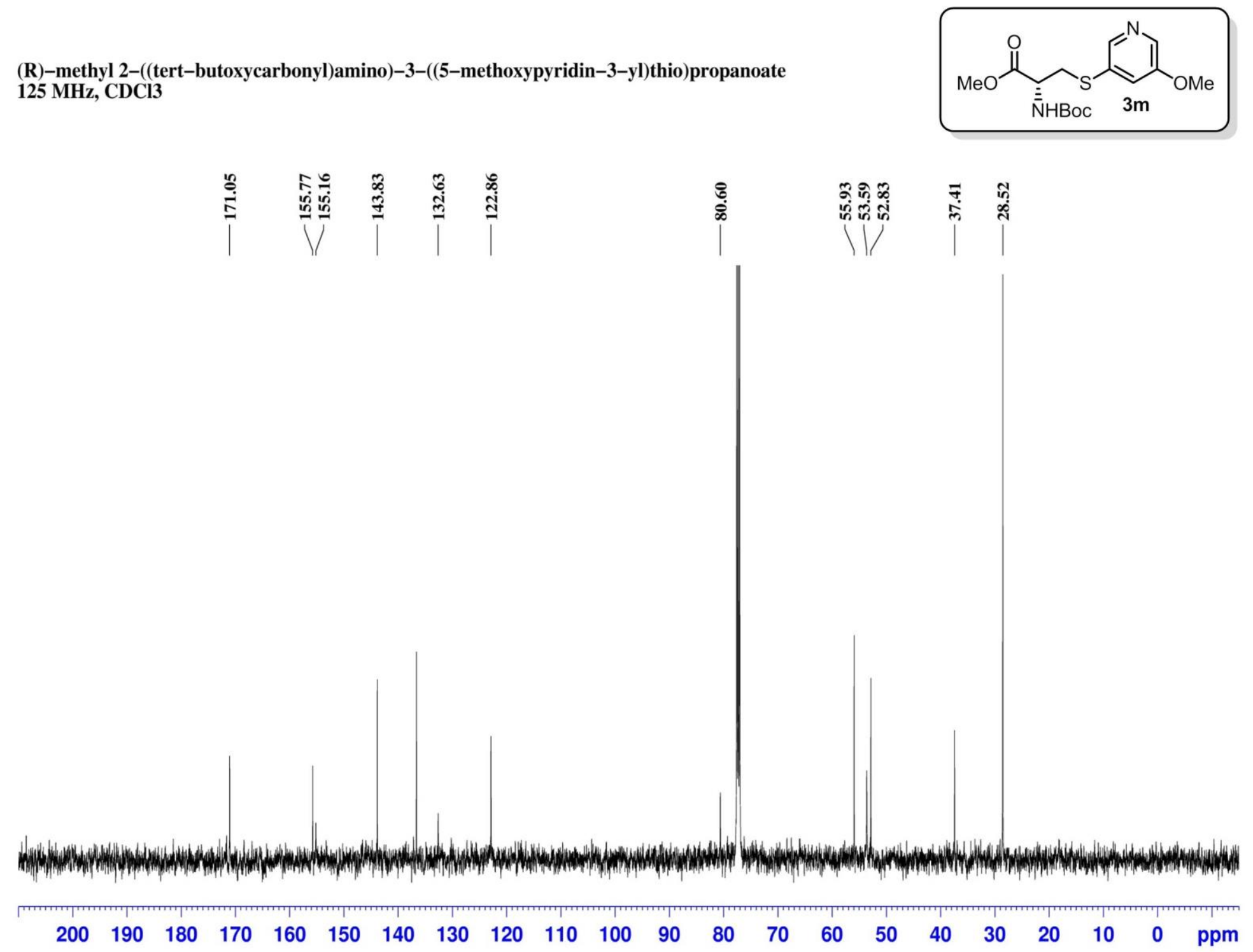
(R)-methyl 2-((tert-butoxycarbonyl)amino)-3-((5-methylpyridin-2-yl)thio)propanoate $125 \mathrm{MHz}, \mathrm{CDCl} 3$
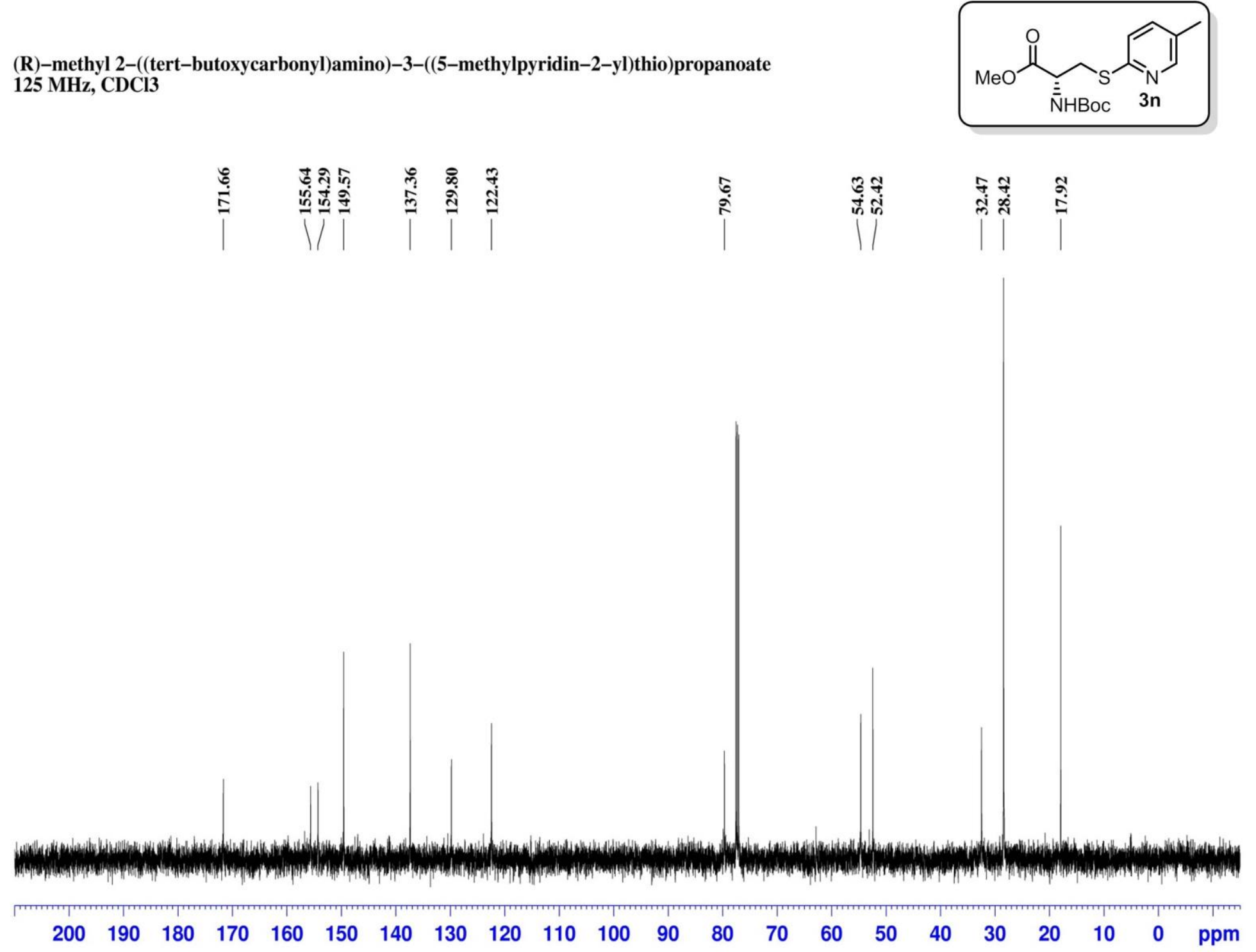
(R)-methyl 2-((tert-butoxycarbonyl)amino)-3-(quinolin-4-ylthio)propanoate $125 \mathrm{MHz}, \mathrm{CDCl} 3$
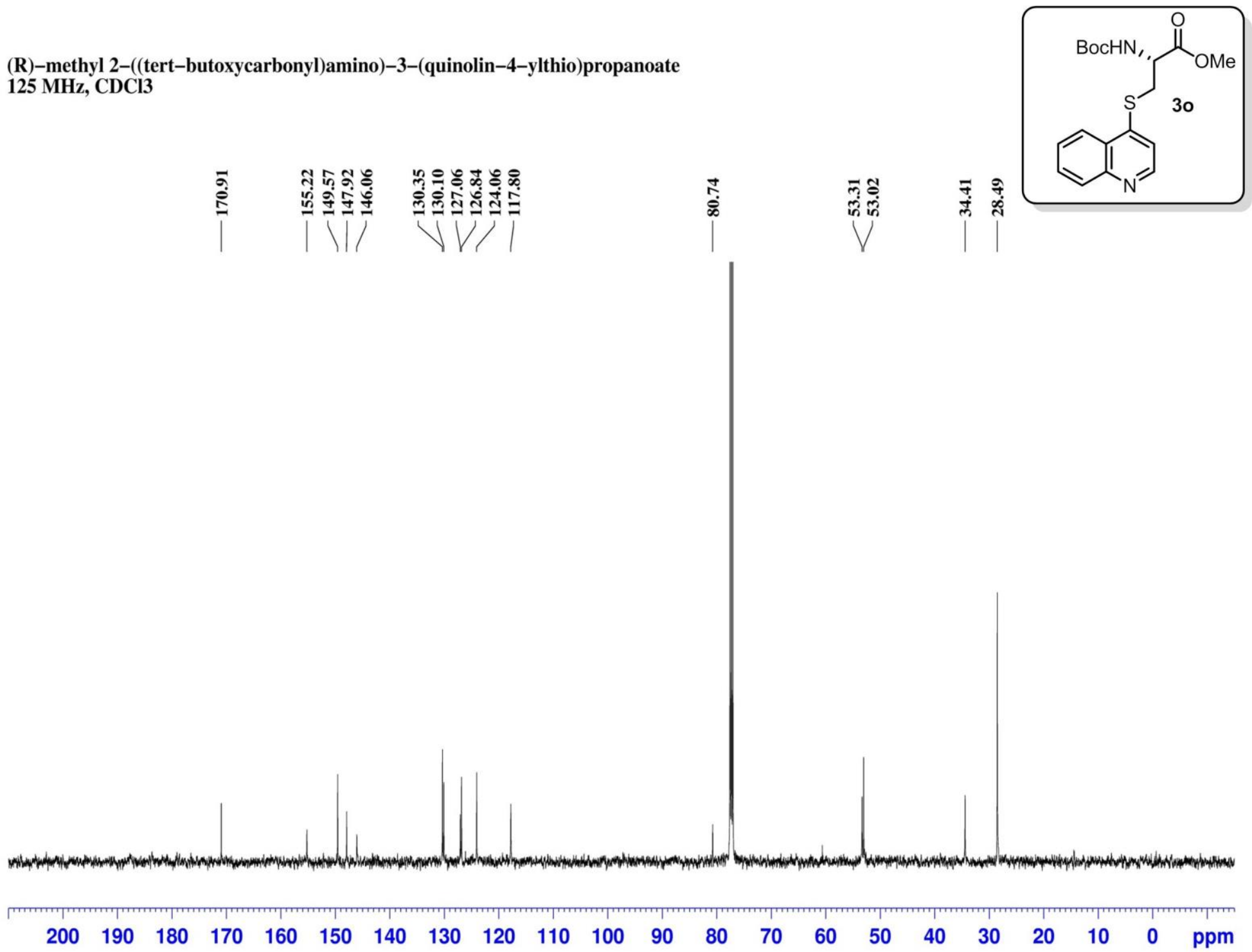
(R)-methyl 2-((tert-butoxycarbonyl)amino)-3-(isoquinolin-4-ylthio)propanoate $125 \mathrm{MHz}, \mathrm{CDCl} 3$
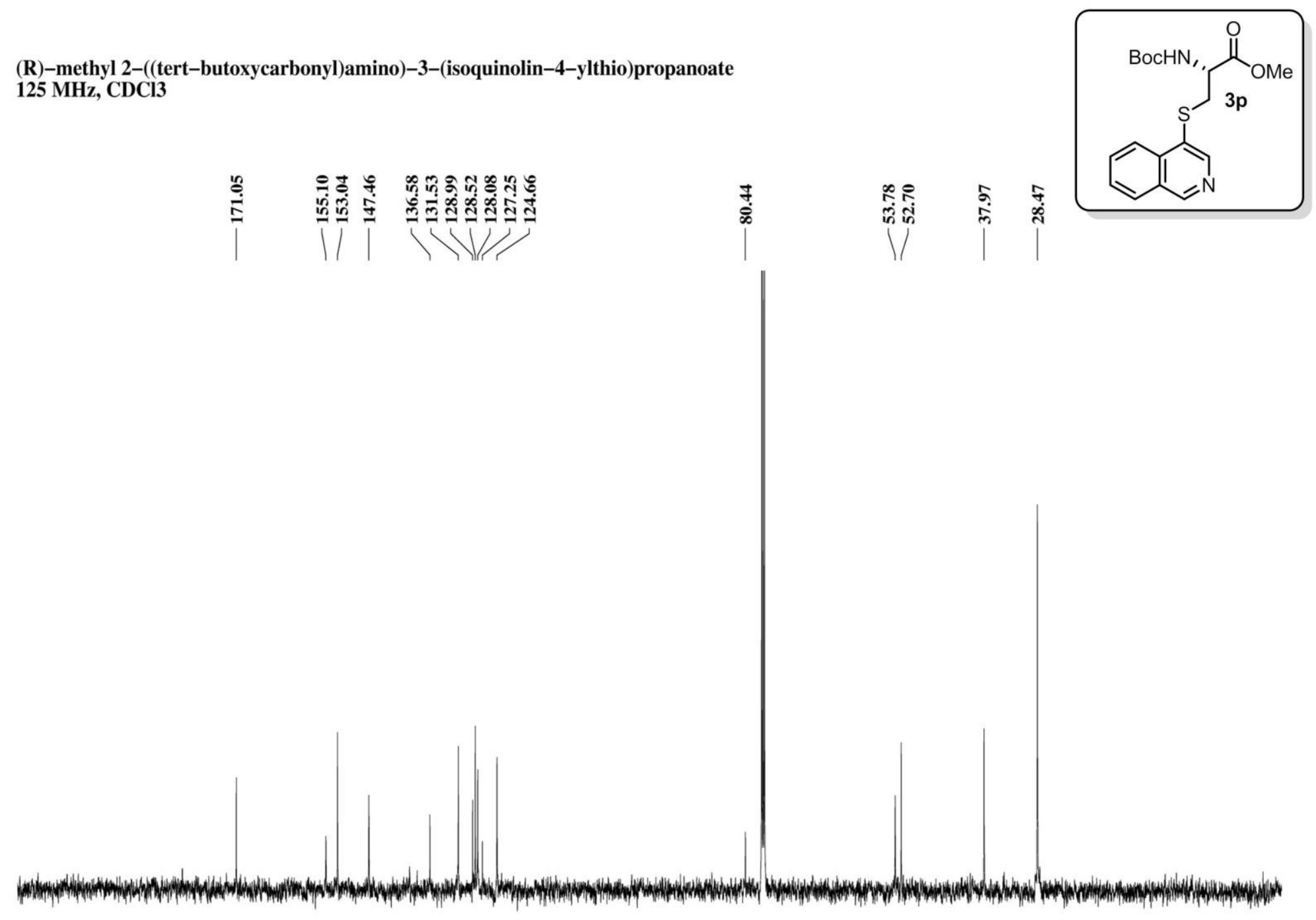

$\begin{array}{lllllllllllllllllllllll}200 & 190 & 180 & 170 & 160 & 150 & 140 & 130 & 120 & 110 & 100 & 90 & 80 & 70 & 60 & 50 & 40 & 30 & 20 & 10 & 0 & \mathrm{ppm}\end{array}$ 
3-(pyrimidin-5-ylthio)hexan-1-ol

$125 \mathrm{MHz}, \mathrm{CDCl} 3$
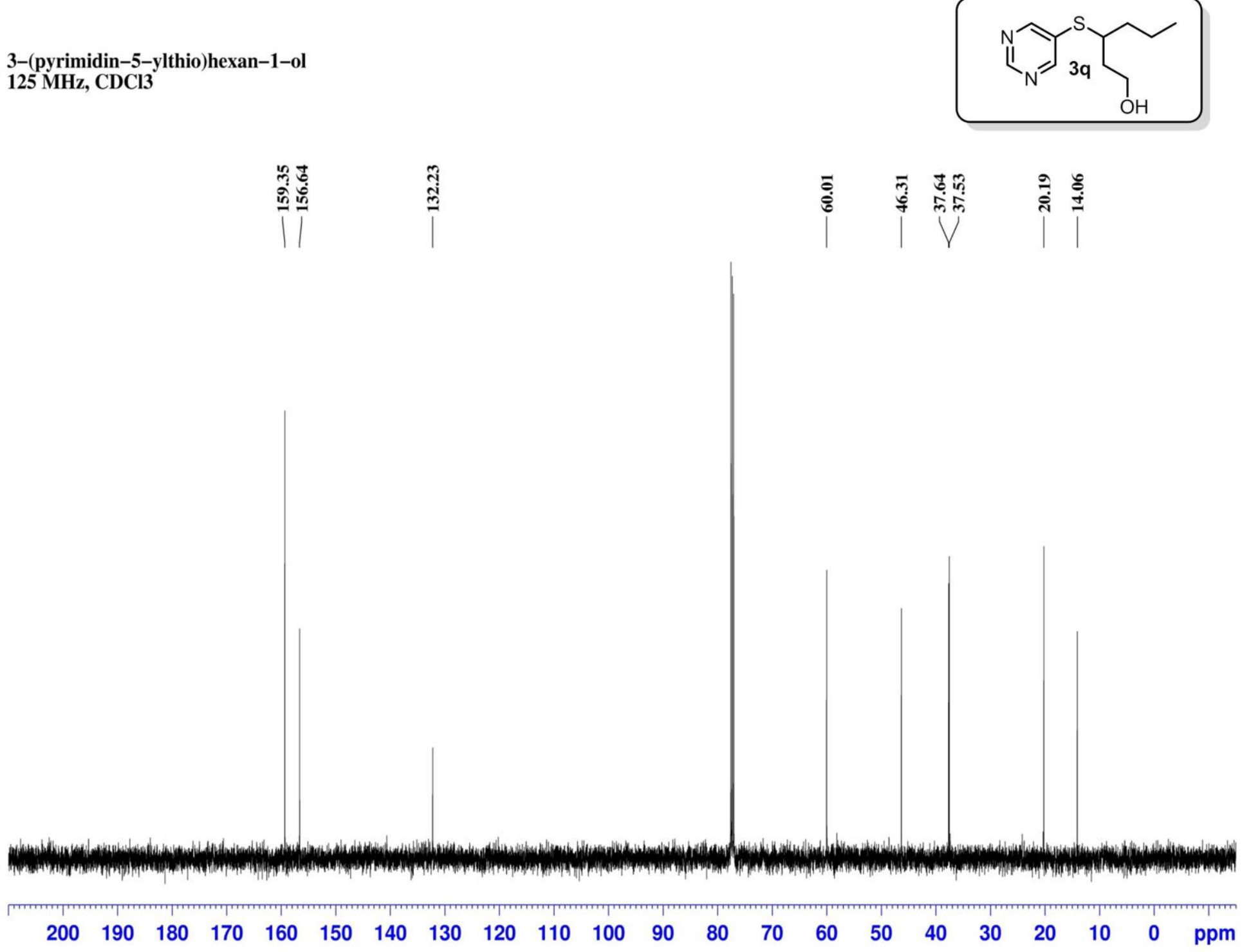
tert-butyl 4-(5-((1-hydroxyhexan-3-yl)thio)pyrimidin-2-yl)piperazine-1-carboxylate $125 \mathrm{MHz}, \mathrm{CDCl} 3$

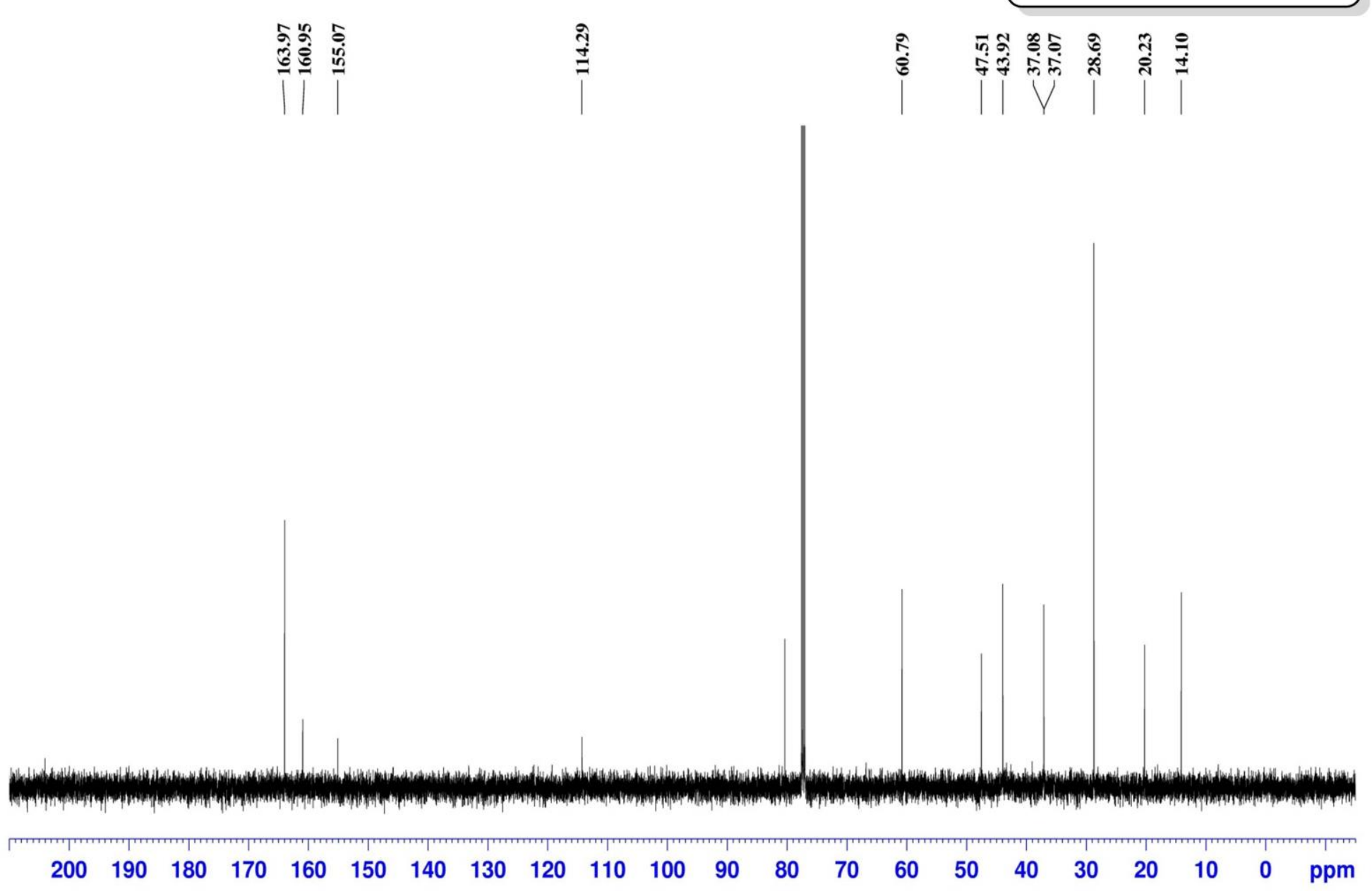


3-((3-methylpyridin-2-yl)thio)hexan-1-ol

$125 \mathrm{MHz}, \mathrm{CDCl} 3$

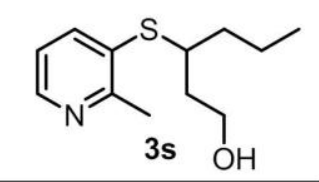

|

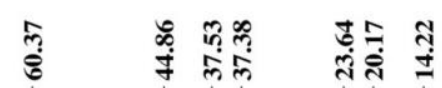

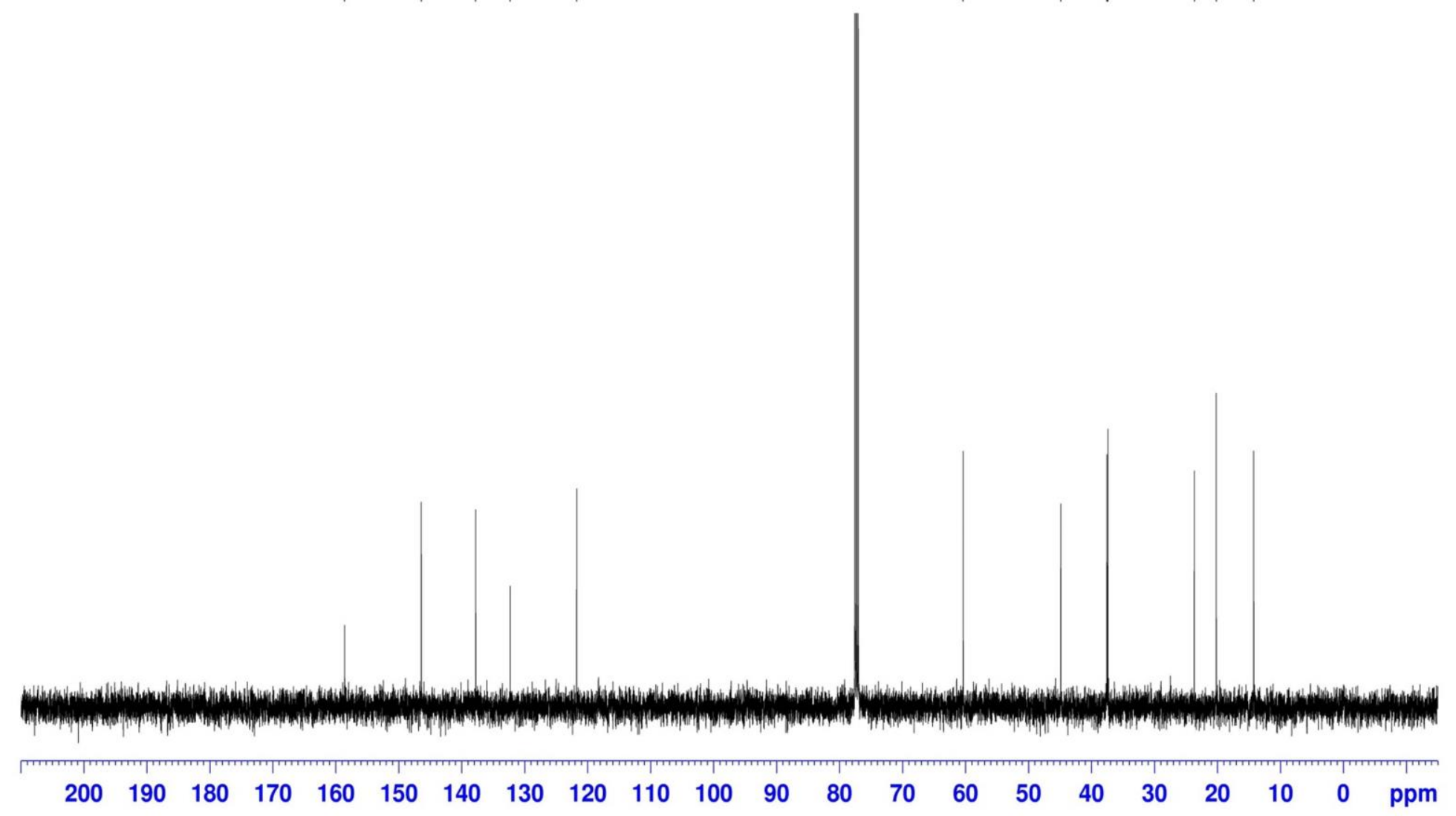


N-(4-((1-hydroxyhexan-3-yl)thio)phenyl)acetamide $125 \mathrm{MHz}, \mathrm{CDCl} 3$
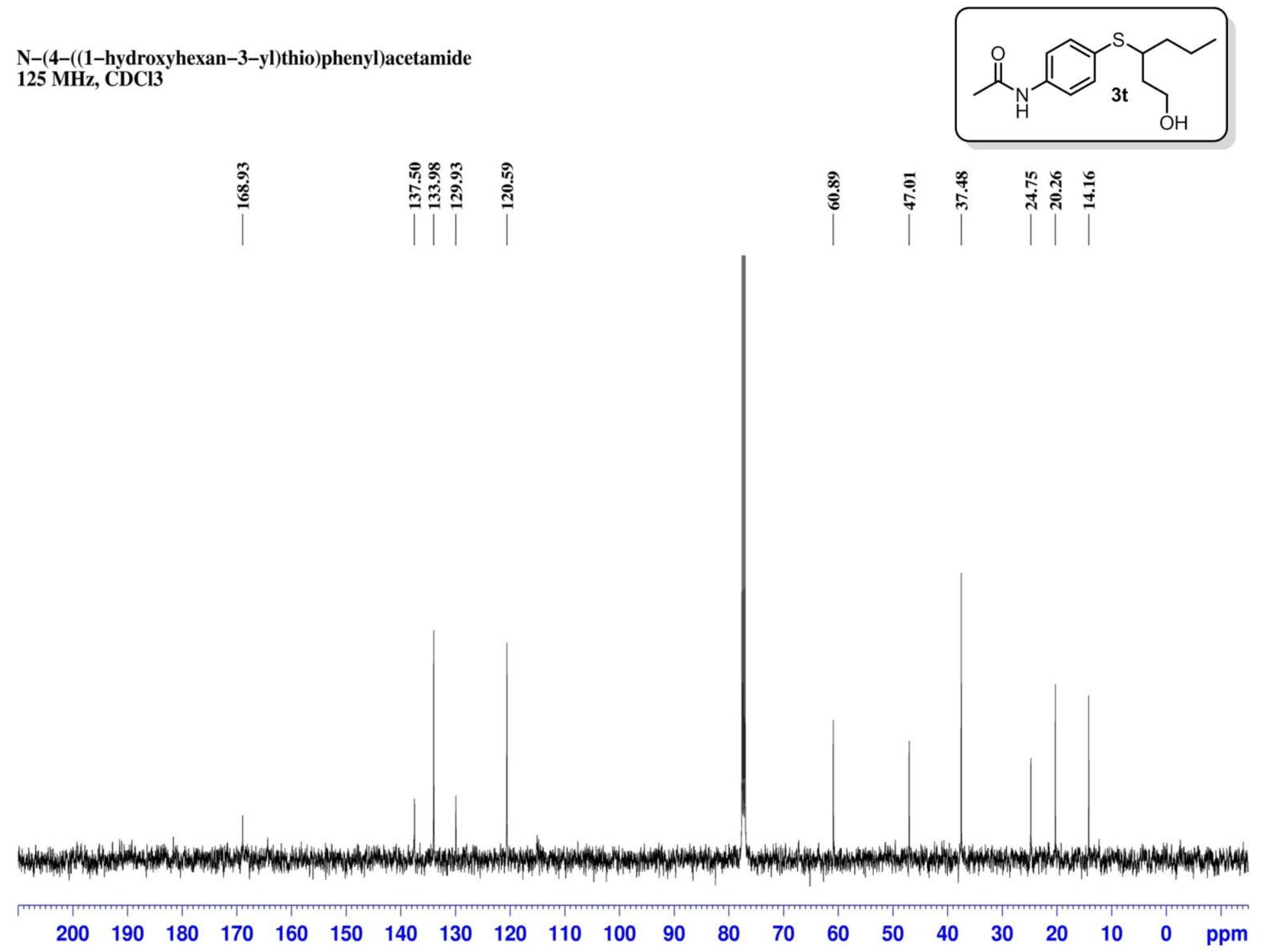
4-((1-hydroxyhexan-3-yl)thio)benzenesulfonamide $125 \mathrm{MHz}, \mathrm{CDCl} 3$
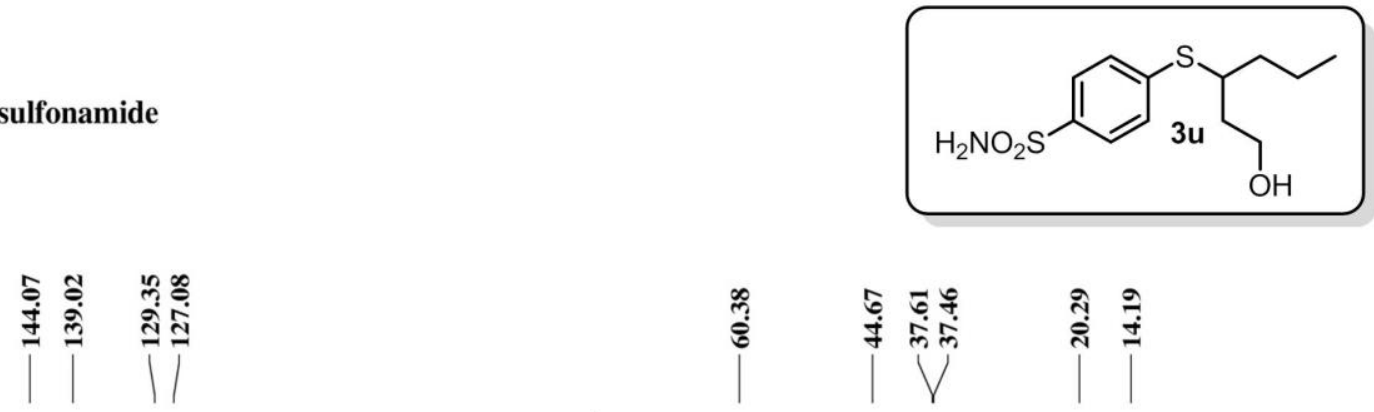

今ి

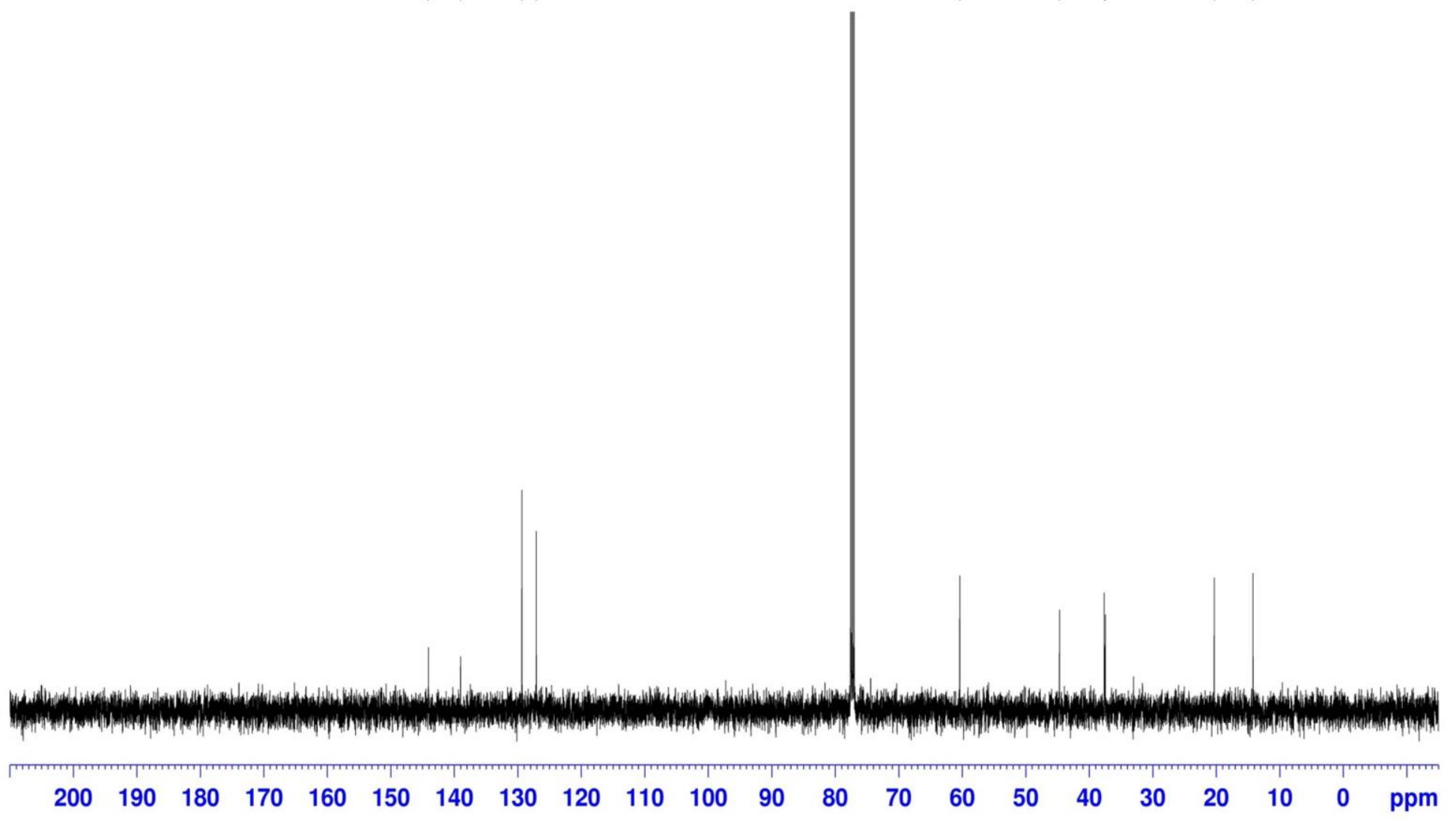


1-(3-(tert-butylthio)phenyl)ethanone $125 \mathrm{MHz}, \mathrm{CDCl} 3$
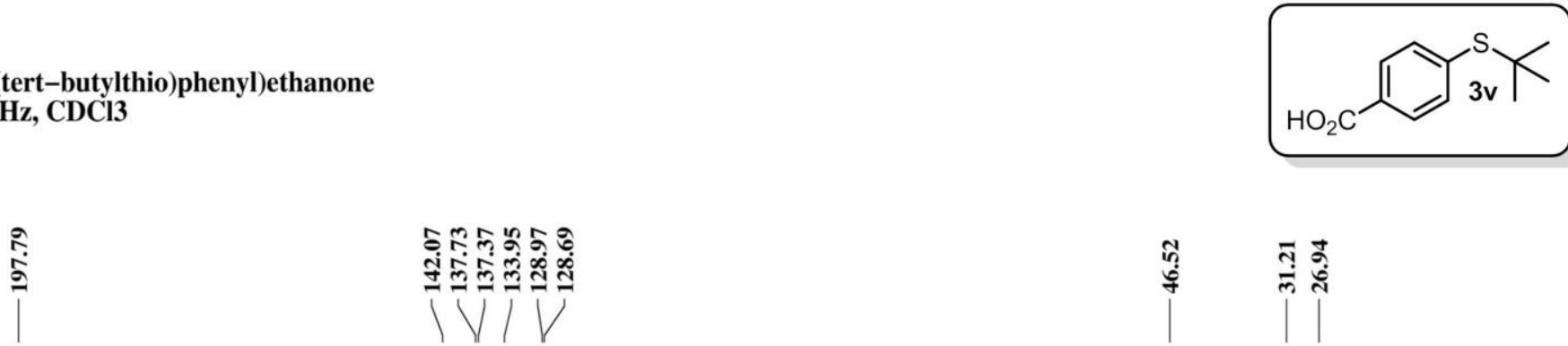

กู้ तु

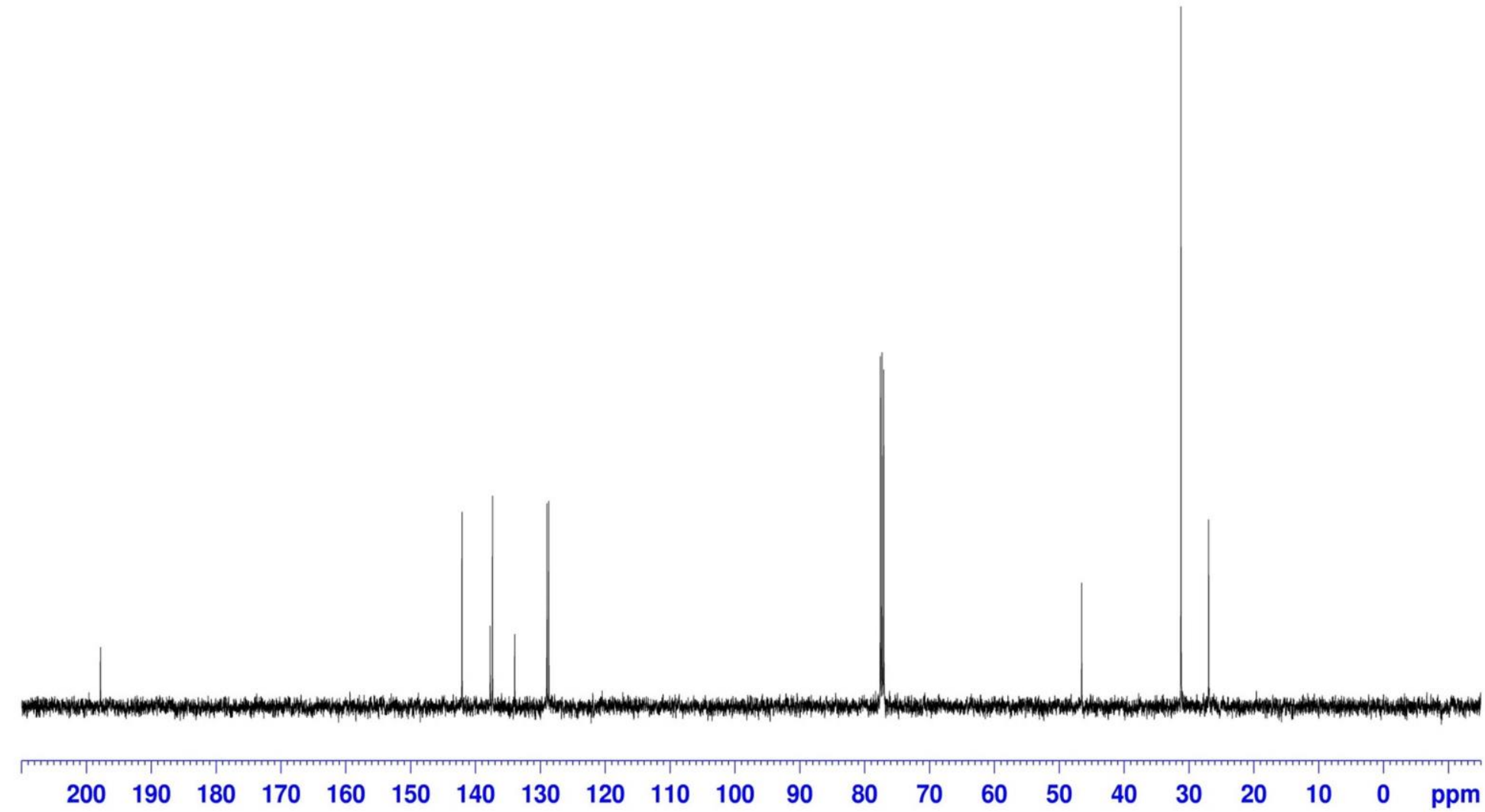


(4-benzylphenyl)(tert-butyl)sulfane

$125 \mathrm{MHz}, \mathrm{CDCl} 3$
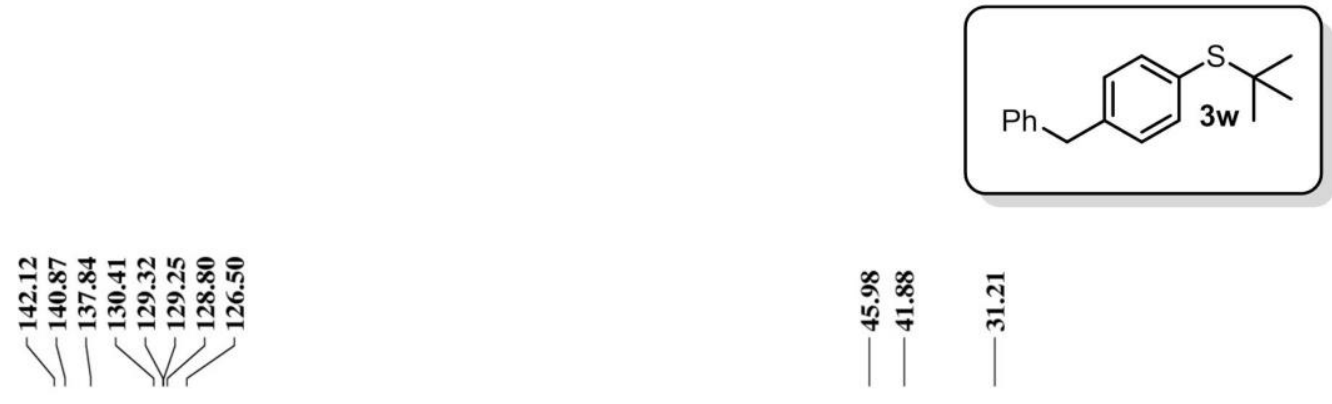

$\mid$

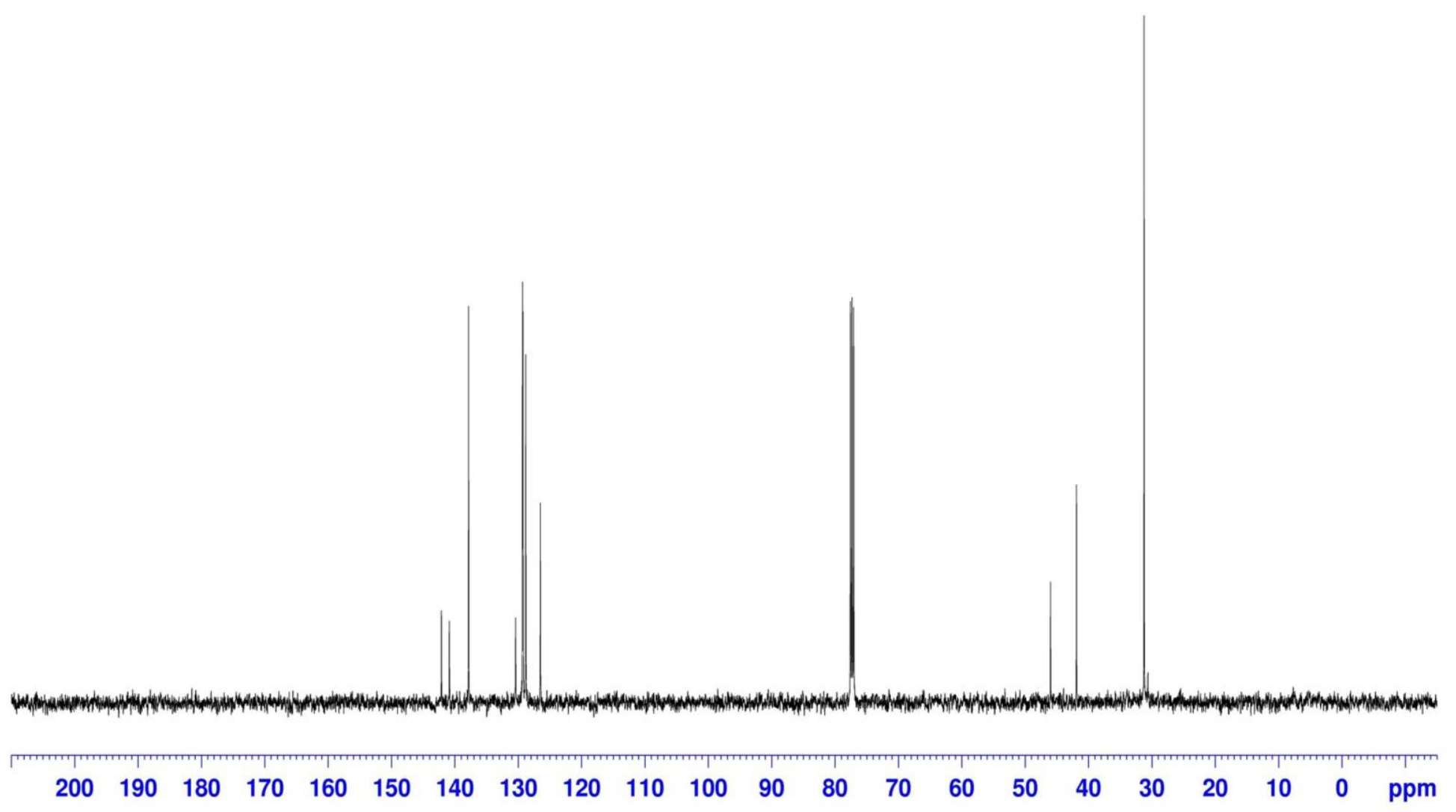



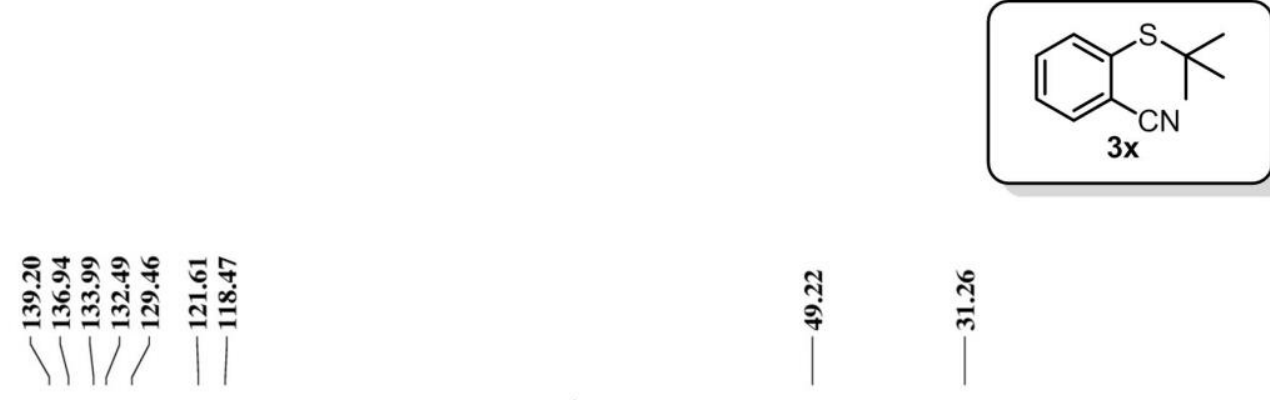

|ุ

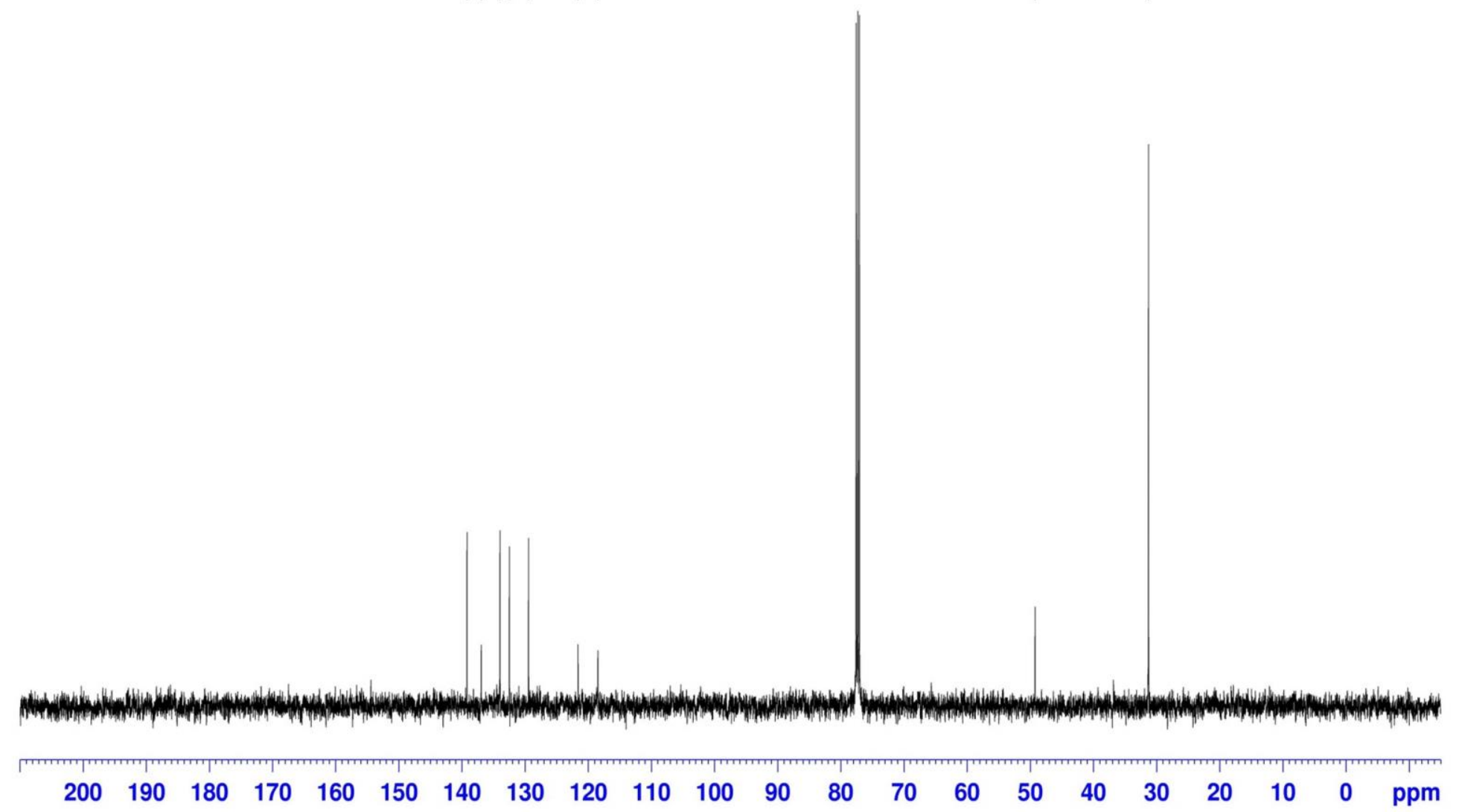




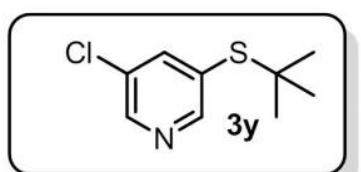

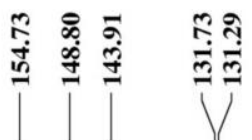

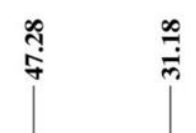

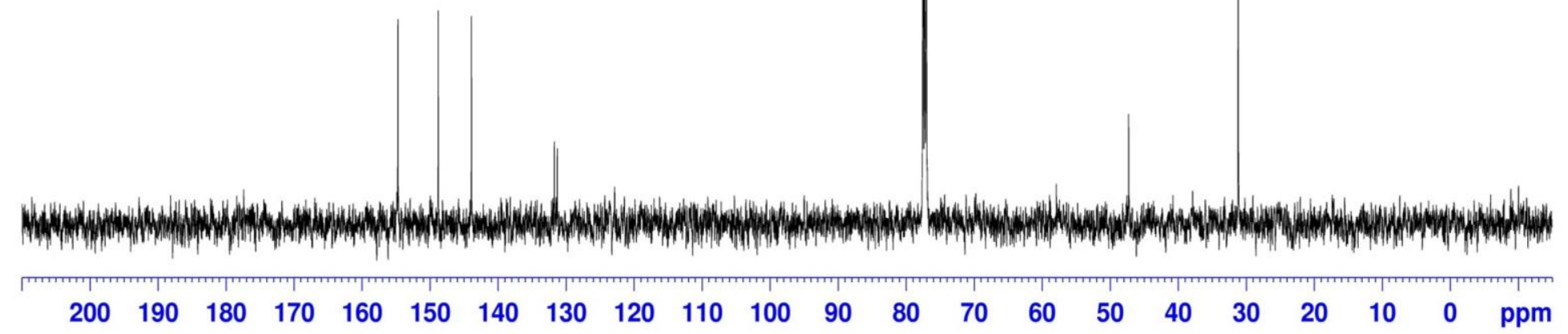


8-(tert-butylthio)isoquinoline

$125 \mathrm{MHz}, \mathrm{CDCl} 3$
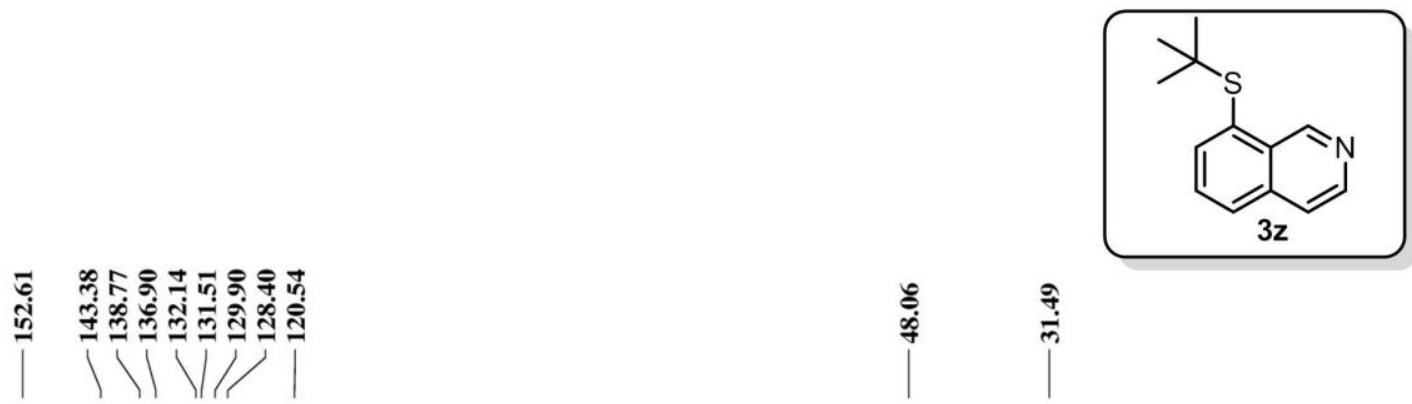

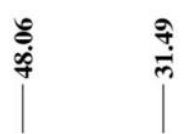

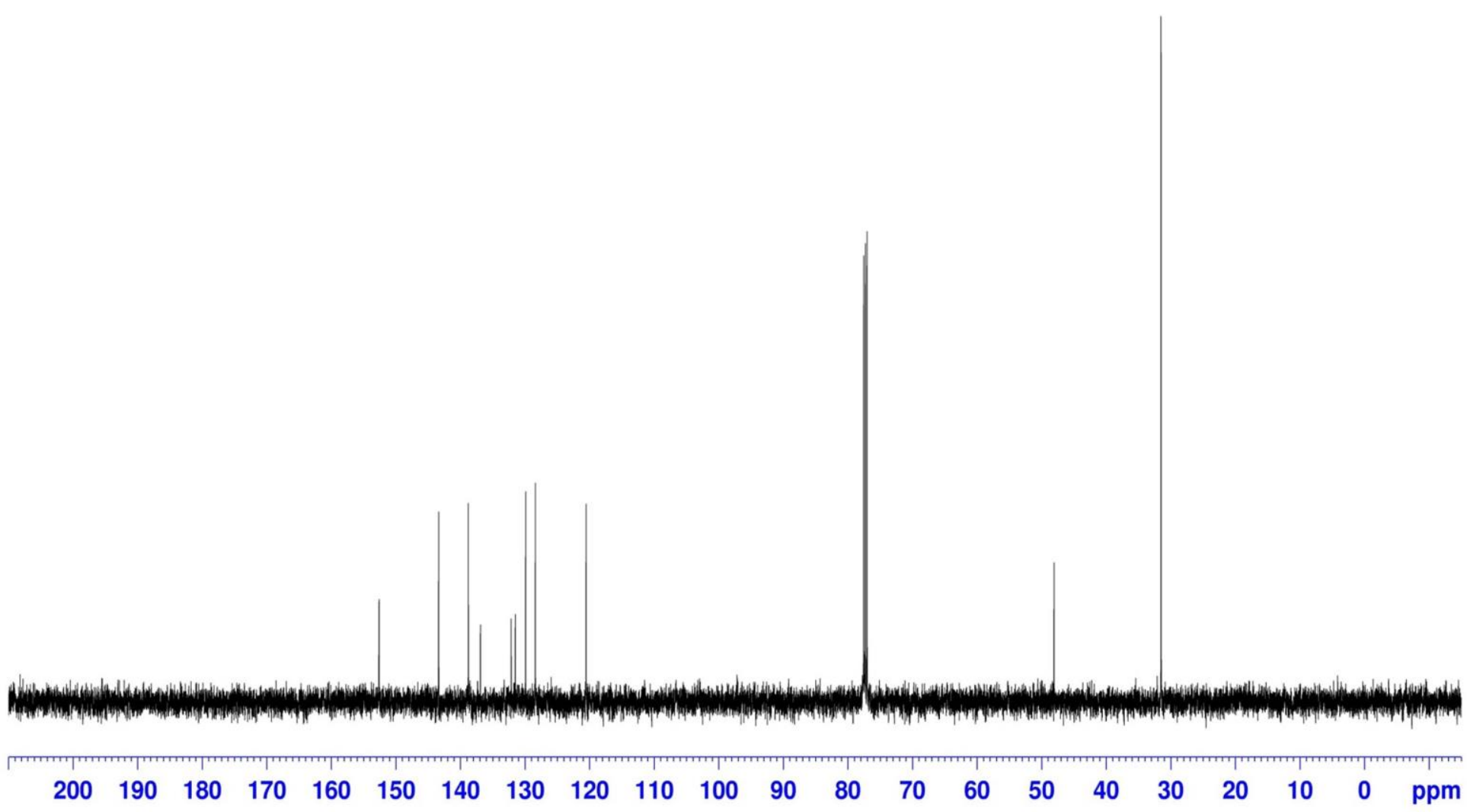




\section{${ }^{19}$ F NMR Spectra of Synthesized Compounds}

$$
\text { 2-fluoro-4-(propylthio)pyridine }
$$

$282 \mathrm{MHz}, \mathrm{CDCl} 3$
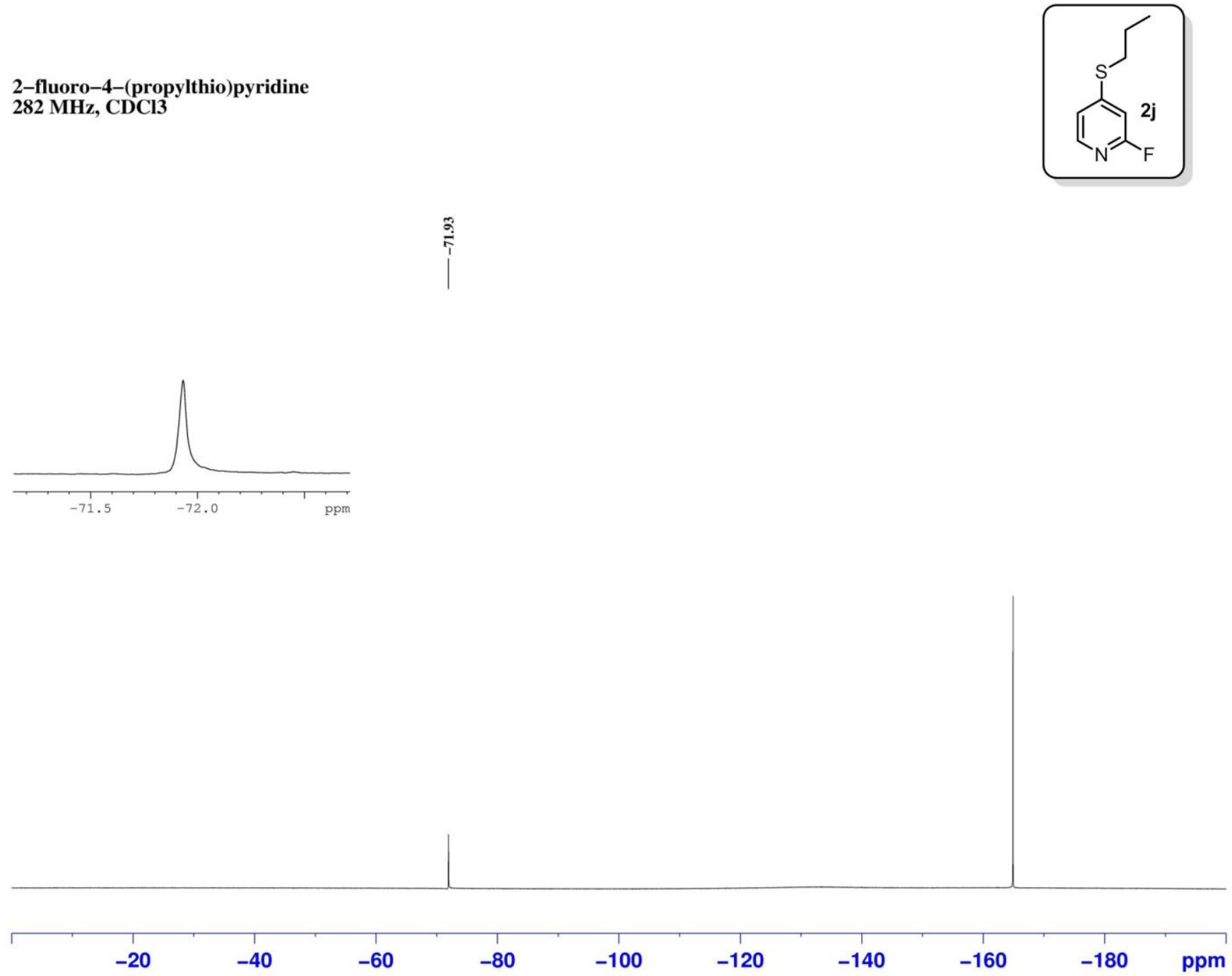
(R)-methyl 2-((tert-butoxycarbonyl)amino)-3-((2-fluoropyridin-4-yl)thio)propanoate $282 \mathrm{MHz}, \mathrm{CDCl} 3$
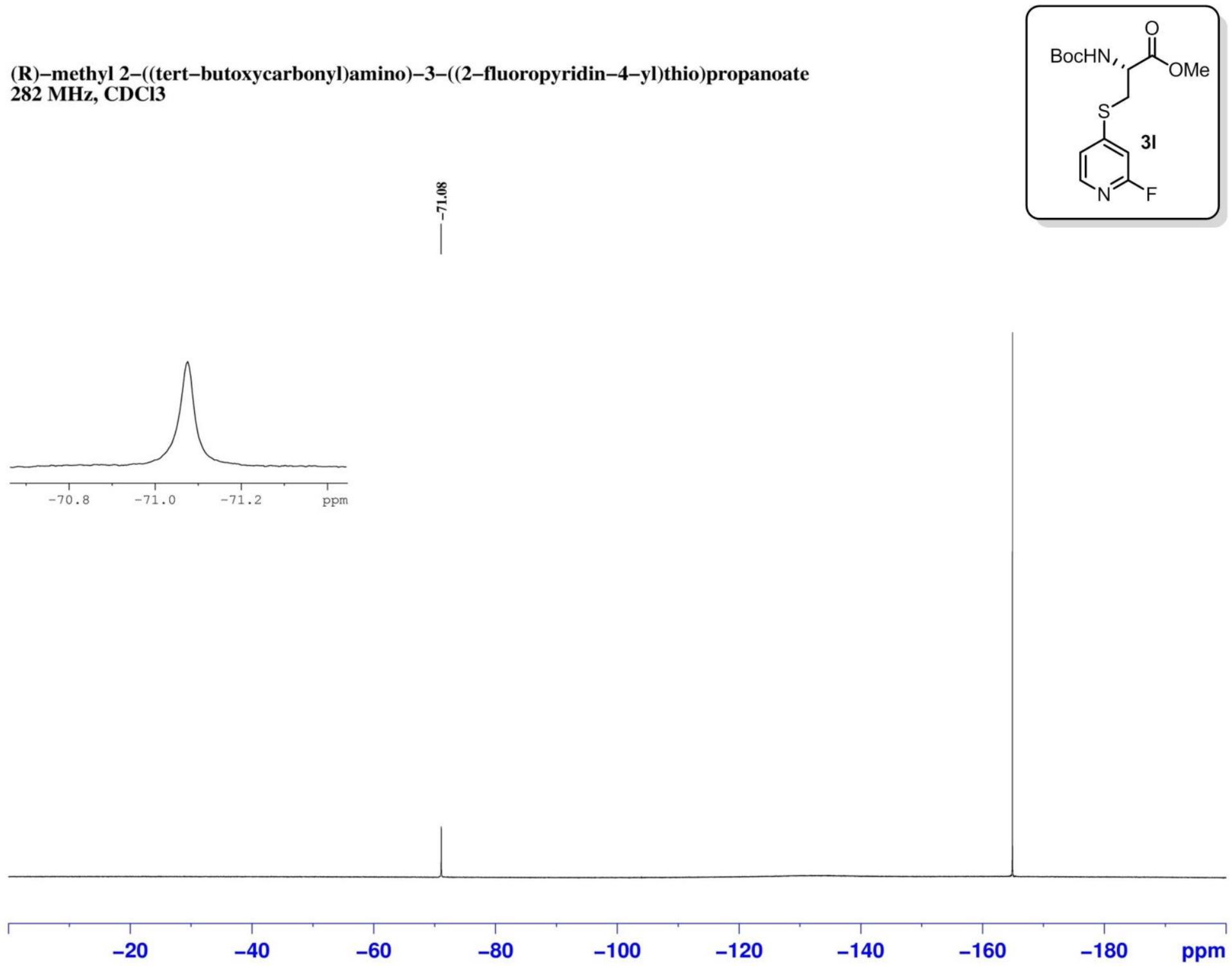